\title{
Immunological and clinical strategies in renal transplantation
}

Citation for published version (APA):

Christiaans, M. H. (1999). Immunological and clinical strategies in renal transplantation. [Doctoral Thesis, Maastricht University]. https://doi.org/10.26481/dis.19991217mc

Document status and date:

Published: 01/01/1999

DOI:

10.26481/dis.19991217mc

Document Version:

Publisher's PDF, also known as Version of record

\section{Please check the document version of this publication:}

- A submitted manuscript is the version of the article upon submission and before peer-review. There can be important differences between the submitted version and the official published version of record.

People interested in the research are advised to contact the author for the final version of the publication, or visit the DOI to the publisher's website.

- The final author version and the galley proof are versions of the publication after peer review.

- The final published version features the final layout of the paper including the volume, issue and page numbers.

Link to publication

\footnotetext{
General rights rights.

- You may freely distribute the URL identifying the publication in the public portal. please follow below link for the End User Agreement:

www.umlib.nl/taverne-license

Take down policy

If you believe that this document breaches copyright please contact us at:

repository@maastrichtuniversity.nl

providing details and we will investigate your claim.
}

Copyright and moral rights for the publications made accessible in the public portal are retained by the authors and/or other copyright owners and it is a condition of accessing publications that users recognise and abide by the legal requirements associated with these

- Users may download and print one copy of any publication from the public portal for the purpose of private study or research.

- You may not further distribute the material or use it for any profit-making activity or commercial gain

If the publication is distributed under the terms of Article $25 \mathrm{fa}$ of the Dutch Copyright Act, indicated by the "Taverne" license above, 


\section{Immunological and clinical strategies in renal transplantation}




\section{Promotor}

Prof. dr. JP van Hooff

\section{Co-promotor}

Dr. PM van den Berg-Loonen

\section{Beoordelingscommissie}

Prof. dr. G Kootstra (voorzitter)

Prof. dr. CA Bruggeman

Prof. dr. FHJ Claas (Leids Universitair Medisch Centrum, Leiden)

Prof. dr. RAMG Donckerwolcke

Prof. dr. G Opelz (Ruprecht-Karls-Universität, Heidelberg, Duitsland) 
$\mathrm{lk}$ heb een steen verlegd in een rivier op aarde. Het water gaat er anders dan voorheen. De stroom van een rivier hou je niet tegen. Het water vindt er altijd een weg omheen.

De Steen (Bram Vermeulen) 


\section{Contents}

Chapter 1: Introduction 9

Chapter 2: HLA-DR matched transfusion: Development of donor-specific $\mathrm{T}$ - and B-cell antibodies and renal allograft outcome $\mathbf{4 3}$

Chapter 3: HLA-class I and II antibodies by ELISA and CDC before and after transplantation $\mathbf{5 9}$

Chapter 4: Flow cytometry: principles and crossmatch protocol $\mathbf{8 1}$

Chapter 5: No advantage of flow cytometry crossmatch over complementdependent cytotoxicity in immunologically well-documented renal allograft recipients 99

Chapter 6: Donor-specific antibodies after transplantation by flow cytometry. Relative change in fluorescence ratio most sensitive risk factor for graft survival 117

Chapter 7a: Use of tacrolimus in renal transplantation 137

Chapter 7b: Pharmacokinetics of tacrolimus and mycophenolic acid (MPA) following the administration of a tacrolimus-based regimen in combination with mycophenolate mofetil (MMF) in kidney transplantation 143

Chapter 7c: Dosing and management guidelines for tacrolimus in renal transplant patients $\mathbf{1 5 1}$

Chapter 8: Multivariate analysis of factors related to immediate and long-term clinical outcome in cadaveric renal transplantation; a single-center study 161

Chapter 9: General Discussion 195 
Samenvatting 221

Dankwoord 227

Curriculum vitae 231

Publicatielijst $\mathbf{2 3 3}$ 

Chapter 1

\section{Introduction}




\section{Introduction}

Adequate renal function is essential to sustain life. Patients with end-stage renal disease (ESRD) therefore need renal replacement therapy. Essentially, there are two alternatives: dialysis or renal transplantation. As short-term treatment, to survive a period of acute renal failure, hemodialysis has been successful since $1945^{1}$ and peritoneal dialysis since $1946^{2}$. A major problem with dialysis is the need for a permanent access. Therefore, chronic replacement therapy by hemodialysis has only been possible since $1960^{3}$, because at this timepoint the Scribner shunt became available as permanent access ${ }^{4}$. Patients who receive a kidney transplant have a higher quality of life, a lower risk of death ${ }^{6}$, and a less costly form of therapy ${ }^{7}$ than those who are treated with dialysis. Therefore, renal transplantation is the preferred treatment for patients with end-stage renal failure.

The first successful human renal allograft was transplanted in $1954^{8}$. Transplantation of a kidney from a living donor is possible due to the fact that the kidney is a paired organ and that one kidney is sufficient to provide adequate function for both donor and recipient. Moreover, because of the availability of dialysis, a patient with end-stage renal failure can be kept alive until the time of transplantation as well as during the period after transplantation until the renal graft has regained function. These facts are what account for the rapid development of renal transplantation compared to other organ transplantations.

The surgical procedure became standardized quite early and no major changes have been made since. While transplantation was initially performed with kidneys from living donors, kidneys from post-mortem (cadaveric) donors are currently also grafted.

It soon became apparent that, except in the case of identical twins, allografts are generally rejected. This stimulated the study of the immune sytem, and drugs were tested for the prevention of rejection. The first drugs used clinically were 6-mercaptopurine/azathioprine in combination with high dosages of corticosteroids. Later on, antilymphocyte globulin (ALG) was used for the first days after transplantation. With this immunosuppressive therapy the rejection process could be suppressed, but the result was a high morbidity rate, due to opportunistic infections. A major improvement was the introduction of cyclosporine A (CyA) in the late 1970s. In particular, the cumulative dose of steroids needed in conjunction with the use of CyA was markedly reduced, leading to a decrease in morbidity. Recently, newer drugs have been introduced, and others are at the clinical trial stage.

Study of the immune system had some early success as well. Incompatibility for 
the $\mathrm{AB} 0$ blood group was shown to result in a high rate of acute rejections in renal transplantation. In the late $1950 \mathrm{~s}$ and $1960 \mathrm{~s}$, another important antigen system, the major histocompatibility complex (MHC), was discovered ${ }^{10.14}$ and shown to be important in transplantation.

By providing compatibility for the human histocompatibility antigens between donor and recipient, one expected the results of renal transplantation to improve. Due to the extreme polymorphism of the HLA system, this was not possible within one center. Therefore, van Rood made a proposal for international collaboration via an organ exchange organization ${ }^{15}$. Eurotransplant was the first organ exchange organization in the world. It assists in the allocation of organs for Austria, Germany, Belgium, Luxemburg, and the Netherlands. Since then, in other parts of Europe and in the rest of the world, many such organ exchange organisations have been established (UK transplant, France transplant, UNOS, etc.).

Nowadays, renal transplantation is the first choice of therapy for every suitable patient with end-stage renal failure.

Worldwide, more than 400,000 renal transplantations have been reported ${ }^{16}$, most of them with cadaveric donor grafts. In the Netherlands, more patients are maintained by a successful transplant than by any other form of treatment (hemodialysis or peritoneal dialysis); over 4000 patients are alive today with a functioning graft $^{17}$. The success of renal transplantation and a simultaneous lack of success in increasing the number of cadaveric donors has created a supply-demand crisis.

A clinical renal transplantation program involves teamwork in which transplant immunologist, surgeon, and nephrologist participate. Generally, the surgeon is responsible for the donor program and the surgical procedure. The nephrologist is more involved in patient selection, pre- and postoperative care, and the handling of immunosuppression. The transplant immunologist is in charge of the transfusion protocol, antibody screening, HLA matching, and crossmatching. Various relevant aspects of these disciplines will be discussed in the following sections.

\section{Surgical technique, preservation, and donor and recipient selection}

The surgical procedure of kidney transplantation is standardized. The graft is placed retroperitoneally in the iliac fossa. The renal artery is anastomosed end-toside to the external iliac artery or end-to-end to the hypogastric artery. The renal vein is anastomosed to the iliac vein. The ureter of the kidney is anastomosed to the urine bladder. The whole procedure takes about 2-3 hours, of which 25-40 
minutes are needed to make the anastomosis of the blood vessels.

\section{Ischemia times}

When a donor kidney is procured, the blood vessels have to be clamped and the kidney becomes 'ischemic'. The 'first warm ischemia time' (WIT1) is the time from the end of blood perfusion of the kidney until the start of the flush with cold preservation fluid. When WIT1 ends, 'cold ischemia time' (CIT) starts. This is defined as the period during which the graft is kept on ice. In the operating room the graft is taken out of the preservation solution and the 'second warm ischemia ime' (WIT2) staris. WIT2 ends when the graft is reperfused by recipient blood.

During each of these periods, the graft is exposed to ischemic damage, which is worsened by the production of free oxygen radicals during reperfusion ${ }^{18}$. This may result in a temporary dysfunction of the graft after transplantation, or delayed graft function (DGF), also wrongly called acute tubular necrosis (ATN). There are other factors, in addition to length of ischemia and reperfusion ${ }^{19.20}$, that contribute to induction of DGF/ATN, e.g., type of preservation solution ${ }^{21}$, suboptimal hydration of the recipient ${ }^{2}$, preformed anti-HLA antibodies ${ }^{23}$, and quality of the donor graft $^{24}$,

\section{Preservation}

When a kidney is removed from the donor, it is perfused with a preservation solution to flush out donor blood and to keep it viable. After the flush with preservation fluid, essentially two methods of preservation are used: cold storage and machine perfusion. In cold storage, the flushed graft is kept on ice until transplantation. In machine perfusion, the graft is continuously perfused with fluid. With the improvement in preservation fluids, the results of cold storage have improved to such a point that they are now comparible with those of machine perfusion. Cold storage is preferred because of its ease of use and lower costs.

It is important to consider the following aspects in the composition of preservation fluids: (1) osmolarity, (2) oxygen radical scavengers, (3) viscosity, (4) preservation of physiological $\mathrm{pH}$, (5) delivery of nucleotide precursor for improvement of rapid restoration of ATP resynthesis after reperfusion (e.g., adenosine), and (6) prevention of blood cell trapping in the graft. The first preservation solution that was used on a large scale was Collins'. Currently, three solutions are commonly used for cold storage: Euro-Collins (EC, a modification of Collins) $)^{25}$, University of Wisconsin (UW) solution ${ }^{26}$, and histidine-tryptophan-ketoglutarate (HTK) ${ }^{27}$. In controlled trials, UW has been proven to be superior to EC in lowering DGF and in improving graft survival ${ }^{21}$; however, it is much more expensive. HTK was originally used as a cardioplegic solution, but it was later shown to be an effective preservation fluid for short-term $\left(<24\right.$ hour) preservation ${ }^{28}$. Because of its strong 
buffer capacity, low viscosity, and relatively low costs, it has been the preferred preservation solution in our center for non-heart-beating donor grafts. Recent experimental studies have suggested that for kidneys with prolonged ischemia, machine perfusion is preferable because it counteracts intrarenal vascular resistance, delivers oxygen and nutrients, and removes metabolites from the graft ${ }^{20}$. This should result in better microcirculatory integrity and a lower DGF rate. Moreover, machine perfusion makes these kidneys accessible for viability testing. In the early years of transplantation, the maximum period of cold storage was about 8 hours. By improving preservation solutions, the preservation period has been extended to over 50 hours.

\section{Donors}

The first renal allografts were from living related donors. Later on, cadaveric (post-mortem) and living unrelated donors were also used. In the USA, many transplantations were initially performed with grafts from living donors, while in the Eurotransplant region the majority were performed with cadaveric donor kidneys. Financial and geographical factors may explain the difference. For a long time in the USA, the costs of dialysis were not refunded by insurance companies, and in many areas there was no local dialysis centre due to the low population concentration. Both factors gave rise to a strong pressure on transplantation with a kidney from a related donor.

Because of the shortage of cadaveric organs in the Eurotransplant region, living donation is now actively promoted. The use of renal xenografts as a donor source has not yet reached clinical practice.

Due to the discrepancy between donor supply and demand, which has resulted in growing waiting lists, donor criteria have become less strict. Nowadays, even elderly donors with comorbidity are accepted, resulting in more so-called 'marginal' or 'sub-optimal' donors. There are reports suggesting that the use of non-optimal grafts results in a higher DGF rate ${ }^{30}$ and worse kidney function early after transplantation, especially when cyclosporine is added to the immunosuppressive regimen ${ }^{31}$.

Transplantation with cadaveric donors is usually performed with brain-dead donors. These donors have irreversible brain damage and have intact circulation. Causes of brain death include trauma, intracerebral bleeding, cerebrovascular accident, poisoning, and cerebral tumors. Another category of cadaveric donors includes those who have died due to cardiac arrest, the non-heart-beating (NHB) donors. In contrast to brain-dead donors, perfusion in NHB donors is not intact, which leads to more ischemic damage. Post-transplantation, these grafts have a high DGF rate and a high rate of never-functioning grafts ${ }^{32}$. However, the intermediate-term graft survival and renal function have been shown to be comparable to those with heart- 
beating post-mortem grafts $^{32,33}$.

Another solution to the donor shortage is the use of living unrelated donors. The early immunological complications may be better managed with newer immunosuppressives. The fact that there is less injury to these grafts implies better long-term results. Terasaki et al. have reported that using living unrelated donors as a source of renal grafts results in a better 3-year graft survival than using grafts from cadaveric donors $(>80 \% \text { vs. } 70 \%)^{34}$.

\section{Recipients}

Potential recipients of a renal graft are thoroughly screened for contraindications. In the first decades of transplantation strict criteria were set. The first recipients were highly selected, generally young recipients with little comorbidity. However, the morbidity rate in these so-called healthy recipients was still high, due to the high steroid dose. Since the introduction of immunosuppressive schemes with low dosages of steroids, morbidity has decreased ${ }^{35}$. Moreover, there is more experience with, and possibilities for, supportive care. Both factors have lead to less strict criteria. Nowadays, many contraindications have become 'relative'. Up until the beginning of the $1980 \mathrm{~s}$, the maximum age for placement on the waiting list was 55 years. In contrast, now it is the biological age that counts instead of the calender age, and patients older than 70 years have successfully received kidney grafts. Nearly $40 \%$ of the recipients undergoing transplantation between 1992 and 1995 at the University Hospital Maastricht were over 55 years of age. Cardiovascular morbidity and diabetes mellitus are also no longer absolute contraindications. Other criteria are still valid, e.g., freedom of infection, absence of (active) malignancy, and negative $\mathrm{T}$ cell crossmatch at the time of transplantation. This 'weakening' of the selection criteria for potential recipients has increased the number of patients on the waiting list considerably.

\section{Antigenic stimulation leading to rejection}

Once perfusion is restored, the recipient's immune system comes into contact with antigens of the donor. To recognize these foreign antigens, they have to be presented to immune cells of the recipient in the context of $\mathrm{MCH}$ molecules. The antigen-presenting cells (APC) may be 'professionals' (e.g., dendritic cells, Langerhans cells, and macrophages) or 'non-professionals' (e.g., B lymphocytes, and endothelial cells). Professionals are good presenters of processed proteins but weak presenters of intact proteins; the opposite is true for 'non-professionals'.

Presentation of the antigen can take place in two ways: by 'indirect' or 'direct' 
recognition. In the indirect pathway, donor antigens are presented to the recipient's T-cell receptor by APCs of the recipient. In the direct pathway, the donor peptide is presented by a donor cell to the recipient's T-cell receptor (TCR). This is schematically depicted in Figure 1.1. Antigens are presented as peptides while placed in the groove of the MCH-molecule. In the indirect pathway, the foreign antigens are generally processed by the APC before they are presented as peptides. Direct recognition bypasses the requirement of processing the antigens by the recipient's APC. The T-cell receptor of the recipient responds directly to the peptide-MHC complex on donor cells. The direct pathway appears to be the predominant form of transplant antigen presentation.

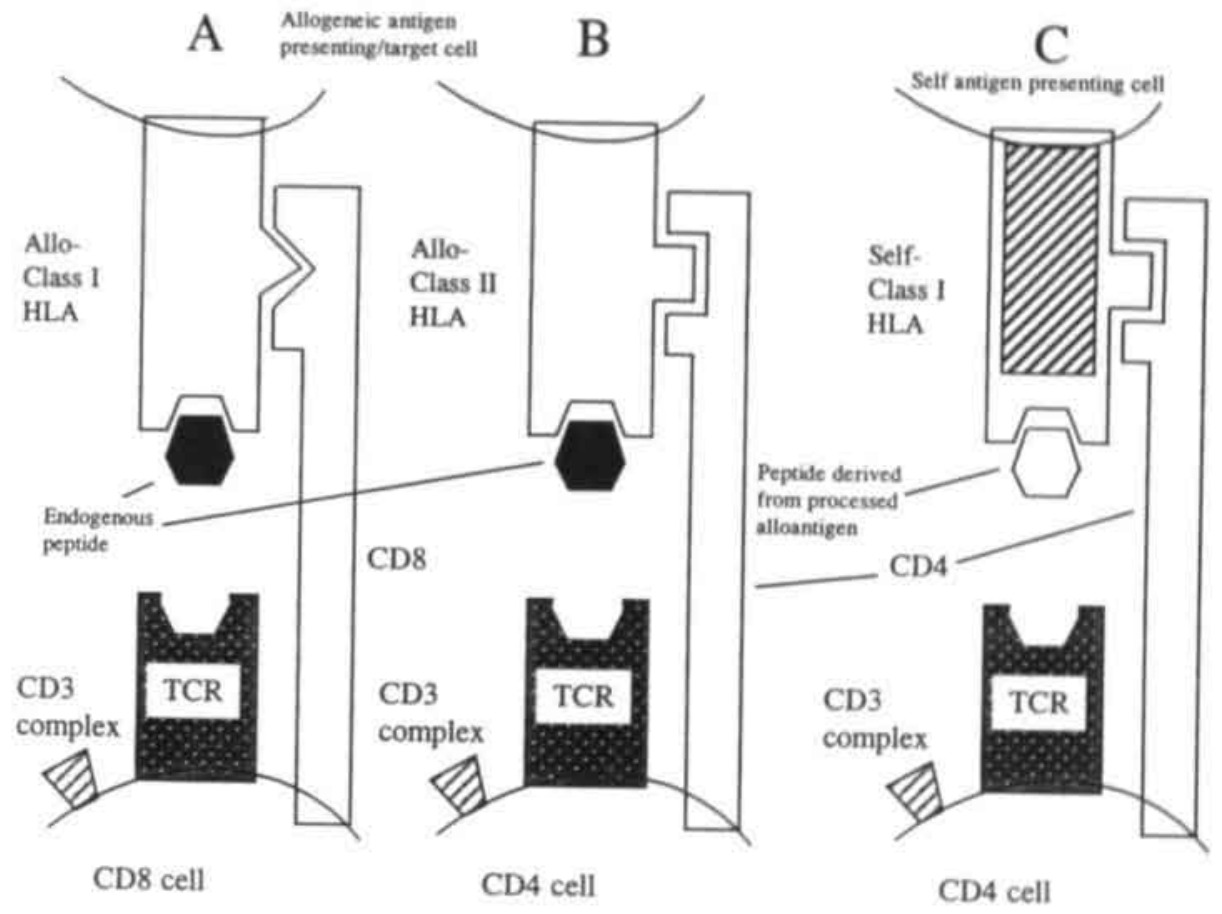

Figure 1.1 Model of allorecognition via the 'direct' $(A+B)$ and 'indirect' $(C)$ pathway.

Presentation of only the antigen in the direct or indirect way is not sufficient for full activation of the immune system. In fact, if a second signal is not provided, anergy or tolerance may be the result. This second signal is needed for stabilization of the cell-cell contact and to provide a co-stimulatory signal. 
One kind of second signal is the binding of the recipient's CD4 or CD8 protein to the presenting cell's MHC molecule in order to stabilize the T-cell-APC interaction. The CD4 protein is present on helper $\mathrm{T}$ cells and binds to the monomorphic component of MHC class II molecules. CD8 protein is present on cytotoxic T cells and binds to the monomorphic component of the MHC class I molecule. Antigenic recognition stimulates redistribution of the TCR with CD3 molecule, together with $\mathrm{CD} 4$ or CD8. This multimeric complex activates intracellular protein tyrosine kinases, eventually leading to activation of the $\mathrm{T}$ cell via calcineurin. Calcineurin is a target molecule for immunosuppressives like cyclosporine and tacrolimus ${ }^{36}$.

Other groups of molecules also play a role as adhesion molecules. They enable recipient lymphocytes to infiltrate the transplant and to bind to APCs. Examples of these molecules are the selectins, the integrins, and the molecules of the immunoglobulin superfamily (e.g., L-selectine and MadCam-1, ICAM-1 and LFA-1, B7, and CD28). Cytokines also play an important role as soluble mediators for communication between cells and for regulation of the immune response. They are produced by lymphocytes, among other cell types. Cytokines may act synergistically, antagonistically, or competitively, depending on the site, timing, and intensity of the stimulus that triggers their release. Cytokines increase the level of costimulatory ligands and are released when there is injury, trauma, or infection. This may explain the increased rate of rejection in these circumstances.

T-helper (CD4-positive) cells consist of at least two subsets: Th1 and Th2 cells. They differ in function and interact with each other via cytokines. Thl cells produce interleukin-2 (IL-2), interferon-gamma, and tumor necrosis factor-beta. Th2 cells primarily produce IL-4, IL-5, IL-6, IL-10, and IL13. Th1 cells favor cellular immunity, while Th2 cells favor humoral immune mechanism. Based on the murine infection model, it was suggested that Thl response is involved in transplant rejection, while $\mathrm{Th} 2$ response is involved in transplantation tolerance.

\section{Types of rejection and their clinical presentation}

Different types of rejection can be discriminated, including hyperacute rejection, acute rejection, and chronic rejection (or chronic allograft dysfunction).

Rejection is suspected when there is a decrease in renal function. Diagnostics like ultrasound, renal perfusion scans, and urine analysis have a low sensitivity and specificity. In the case of clinical suspicion of rejection, a needle core biopsy of the kidney has to be performed. In recent years, there have been consensus meetings on the histological definitions of the different kinds of rejections. This has resulted 
in the Banff classification, the latest revision of which was in $1997^{37}$. Clinical studies have shown good correlations between clinical outcome and the Banff classification.

Hyperacute rejection occurs within minutes after reperfusion of the graft. Within minutes, pink color becomes purple, and later black/hemorrhagic. At that stage, the kidney has to be removed. If this rejection is clinically milder, it presents after transplantation with a decrease in urine output and decreased perfusion on a nuclear scan. Historically, a biopsy is taken 1 hour after reperfusion to diagnose hyperacute rejection. Preformed antibodies directed against donor alloantigens are thought to be responsible for this kind of rejection. These antibodies bind to endothelial antigens and activate complement. Antigen-dependent cellular cytotoxicity may also be involved. Polymorphonuclear leukocytes and platelets are attracted, resulting in thrombi and infiltration. By performing a crossmatch before transplantation, the presence of anti-HLA antibodies can be detected and transplantation cancelled. However, hyperacute rejection is still present in $0.1 \%-1 \%$ of the transplants ${ }^{38}$.

Acute rejection usually occurs within the first months after transplantation, although it can also occur later on. The early type starts within days after transplantation and is called "acute accelerated rejection", an antibody-mediated form of acute rejection. In the classical full-blown case, renal clearance decreases rapidly with sodium retention, causing edema and hypertension, while the inflammation causes enlargement of the graft with pain and fever. Usually the clinical picture is milder, or even subclinical, e.g., in the case of DGF. Histologically, the kidney is infiltrated with mononuclear cells, mainly consisting of $\mathrm{T}$ cells, B cells, macrophages, and large granular lymphocytes. Antibodies play a role in acute rejection episodes, and the presence of anti-class $I$ antibodies are associated with a worse prognosis ${ }^{39,40}$. The graft endothelium is probably the first target of these antibodies. The effector mechanism triggered includes recruitment of polymorphonuclear monocytes and mononuclear cells. Then, cells infiltrate the interstitium. Generally, this infiltration is responsive to anti-rejection treatment, while the vascular damage is more resistant. Repair mechanisms may result in scarring. This scarring, especially in the case of recurrent attacks, may culminate in 'chronic changes' seen in grafts with late failure. Vascular acute rejections often do not respond to increased dose of steroids and have an unfavorable prognosis compared to interstitial acute rejections.

Chronic rejection, or chronic transplant nephropathy, is a process that usually becomes clinically apparent 6 months post-transplantation. It is one of the most important causes of graft $\operatorname{loss}^{41}$. Its cause is multifactorial, including immunological and non-immunological factors ${ }^{42}$. The clinical picture is a slow decrease in renal 
function over months to years with the occurrence of proteinuria. The patient usually does not have complaints, unless due to renal failure. Histologically, a biopsy will show a picture of arteriosclerosis, smooth muscle proliferation, and nephrosclerosis. The glomeruli and small vessels will appear to be the prime site of immunological attack, with secondary tubular atrophy and interstitial fibrosis. Again, the initial damage seems to involve the vascular endothelium. Both humoral and cellular immunity play a role. Chronic rejection is unresponsive to the current immunosuppressives, and no effective treatment yet exists.

\section{Immunosuppression}

Because in the first successful renal allograft procedure the kidney donor and recipient were identical twins, no immunological reaction of recipient against donor kidney occurred. In other combinations, the donor graft is recognized as 'foreign' and will be rejected, unless this is prevented. Therefore, recipients need immunosuppressive drugs to prevent rejection.

In clinical practice, one can divide immunosuppressives into so-called basic immunosuppressives and immunosuppressives for the treatment of rejection. Generally, basic immunosuppression consists of a combination of various drugs. In the early phase after transplantation, dosages are kept at a higher level than they are later. Sometimes, a course of poly- or monoclonal cytotoxic antibodies is added. This is called induction therapy. The application of induction therapy is more popular in the USA than in Europe.

When, regardless of this basic immunosuppression, rejection occurs, antirejection therapy consisting of boluses of high-dose steroids is given. When the rejection is reversed, it is called steroid-sensitive. If the rejection process continues, it is called steroid-resistant. In this case, polyclonal (rATG, ALG) or monoclonal antibodies are prescribed.

Administering steroid boluses carries the risk of complications. Steroid-resistant rejections lead to short-term and long-term graft loss. Therefore, both types of rejection are major endpoints for clinical trials in which the efficacy of various combinations of immunosuppressives is tested.

In the azathioprine era, the rejection rate was much higher, and graft loss due to steroid-resistant rejection was able to be prevented by primary treatment of acute rejection by ATG instead of high dosages of steroids ${ }^{43}$. Although this has not yet been proven in the cyclosporine era, some centres, including ours, treat acute rejections in recipients on a cyclosporine-based immunosuppressive regimen primarily with ATG. 
The first drug clinically used was 6-mercaptopurine, an antiproliferative drug introduced in $1959^{44}$. This was later replaced by a derivative, azathioprine (Imuran, Wellcome, England) because of the latter's better bioavailability and lower myelotoxicity. Azathioprine was used in combination with steroids. The 1-year graft survival with this combination was $\pm 60 \%-70 \%$, while $\pm 70 \%$ of the patients needed more than one treatment for acute rejection ${ }^{46}$. An induction course with anti-lymphocyte globulin (ATG) for the first weeks, in addition to azathioprine and steroids, reduced the number of acute rejections ${ }^{47}$. In the 1970 s, cyclosporine A (Sandimmune, Sandoz/Novartis, Switzerland) was introduced ${ }^{48}$. Its mechanism of action is prevention of activation of $\mathrm{T}$ lymphocytes at the level of cytokine gene transcription (as reviewed by Schreiber et al. ${ }^{49}$ ). Cyclosporine reduced the number of acute rejections, and the amount of corticosteroids could be decreased considerably. While cyclosporine-based immunosuppressive regimens have improved the 1-year graft survival ${ }^{50.52}$, its superiority in terms of graft survival after the first year post-transplant over azathioprine/steroid-based immunosuppressive regimens has not been firmly established. Cyclosporine-based immunosuppression was the standard immunosuppression in most centers up until the mid1990s. Cyclosporine is generally used in combination with steroids (double therapy) or with both steroids and azathioprine (triple therapy). In the Netherlands, cyclosporine/Sandimmune was replaced in 1995 by cyclosporine/Neoral, a new microemulsion formulation. Neoral has a more rapid and consistent absorption as it is virtually bile-independent and this better absorption leads to lower intrapatient variability than Sandimmune ${ }^{53}$.

Recently, two new drugs have been introduced: tacrolimus (Prograft, Fujisawa, Japan) and mycophenolate mofetil (Cellcept, Roche, Switzerland). Tacrolimus is used instead of cyclosporine, while mycophenolate mofetil is generally used together with cyclosporine or tacrolimus instead of azathioprine. In randomized multicenter studies comparing tacrolimus with cyclosporine/Sandimmune (both in combination with steroids and azathioprine), tacrolimus reduced the incidence of acute rejection from $45 \%$ to $30 \%^{54.55}$. In fact, the number of steroid-resistant rejections was halved. Also, in comparison with cyclosporine/Neoral, tacrolimus reduces the number of acute rejections ${ }^{56}$. Replacing azathioprine with mycophenolate mofetil reduces the incidence of acute rejection by $\pm 50 \%$ compared to the combination of azathioprine with cyclosporine and steroids ${ }^{57,58}$ and to the combination of azathioprine with tacrolimus and steroids ${ }^{39}$. Because of the reduction in steroid-resistant rejection, tacrolimus and mycophenolate mofetil are expected to result in improved long-term graft survival. Even newer drugs, recently introduced or under investigation, are monoclonal antibodies against the IL-2 receptor (daclizumab, Roche, Switzerland; basiliximab, Novartis, Switzerland), and sirolimus (Novartis, Switzerland; Wyeth, USA). In Table 1.1, the most common immunosuppressives are mentioned, together with their mechanism of action. In 
Figure 1.2, the site of action of these immunosuppressives in the rejection cascade is depicted. Because various drugs are active on different sites, it is logical to combine various agents.

Table 1.1 Immunosuppressives used in renal transplantation and their mechanisms of action.

\begin{tabular}{ll}
\hline $\begin{array}{l}\text { 6-Mercaptopurine } \\
\text { Azathioprine } \\
\text { Mycophenolate mofetil }\end{array}$ & $\begin{array}{l}\text { purine synthesis inhibition } \\
\text { purine synthesis inhibition } \\
\text { purine synthesis inhibition (selective for lymphocytes) }\end{array}$ \\
$\begin{array}{l}\text { Corticosteroids } \\
\text { byclosporine } \\
\text { Tacrolimus }\end{array}$ & $\begin{array}{l}\text { inhibition of IL-2 synthesis } \\
\text { inhibition of IL-2 synthesis }\end{array}$ \\
$\begin{array}{l}\text { Sirolimus } \\
\text { Daclizumab }\end{array}$ & $\begin{array}{l}\text { inhibition of cytokine signal transduction } \\
\text { Basiliximab }\end{array}$ \\
ATG / ALG / OKT3 & blockade of IL-2 receptor \\
\hline
\end{tabular}

\section{Short- and long-term results}

Complications occurring in the first days post-transplantation are mainly surgical: urine leakage, thrombosis of the graft, and delayed graft function. The latter two complications can be caused by injury to the graft and immunological factors. Thrombosis may be the result of hyperacute rejection, and DGF rate is higher in allosensitized recipient $\mathrm{s}^{23}$. At a later stage, such surgical complications as wound infections and lymphocele may occur.

Non-surgical complications include rejections, infections, and malignancies. The different types of rejections are discussed before. Because of the immunosuppression, patients have a higher risk of infection and malignancies. Not only bacterial infections, but also viral infections can occur. Infections with cytomegalovirus in particular may be life-threatening. A malignancy that is related to infection with Epstein-Barr virus (EBV) is non-Hodgkin's lymphoma, especially in EBV-negative recipients of EBV-positive grafts. This situation is common in young recipients. This lymphoma has the highest incidence in the first year after transplantation, while other malignancies, e.g., skin cancer, have a higher incidence much later. The 1-year patient and graft survival is $\pm 95 \%$ and $\pm 85 \%$, respectively. These percentages fluctuate according to selection criteria for donor (cadaveric, NHB, living-related, age limit), recipient (age limit, comorbidity, transplant history), and histocompatibility matching. 


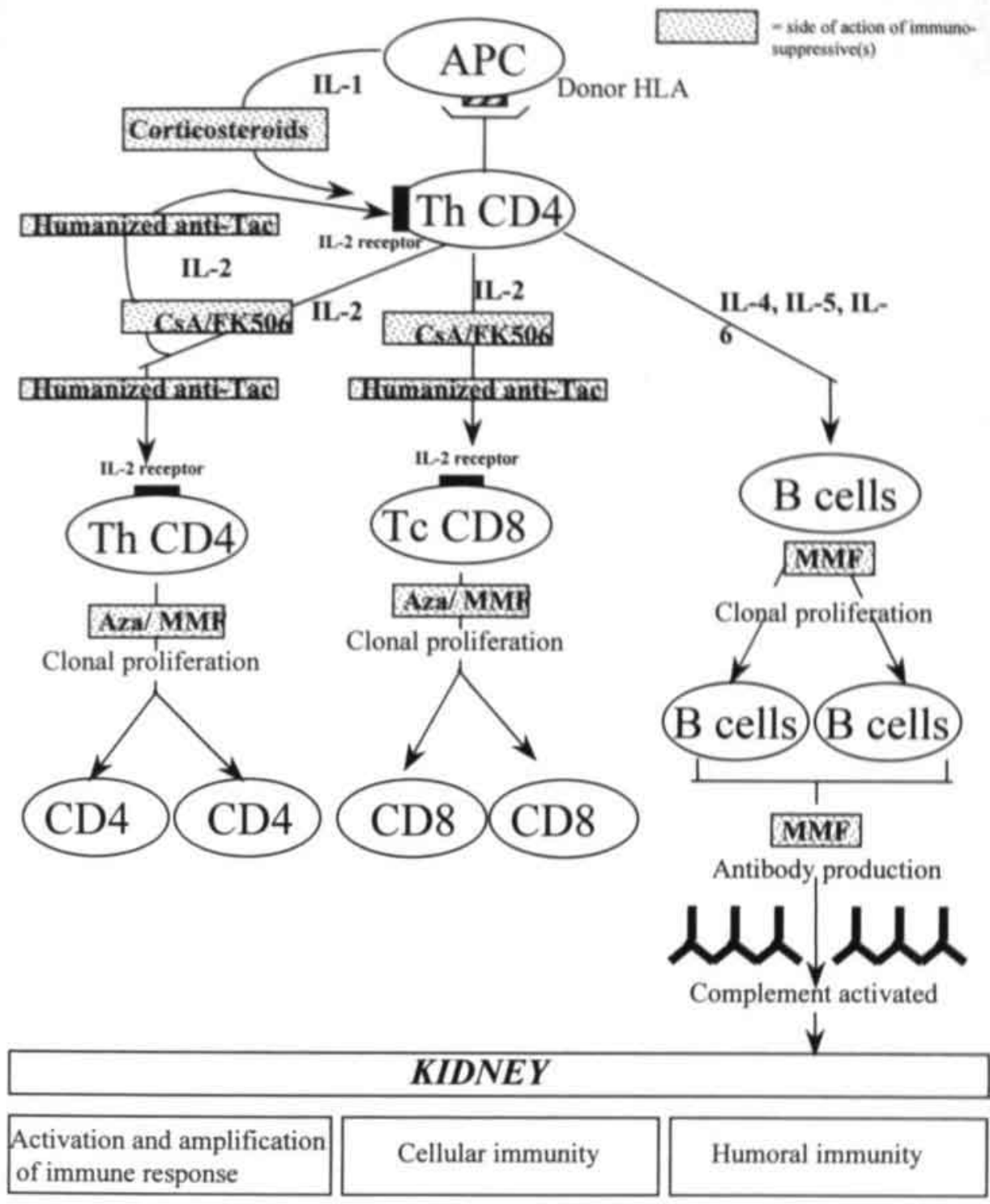

Figure 1.2 Actions of immunosuppressives in the rejection cascade,

Long-term results of renal transplantation are influenced by the selection of recipient and donor, matching for immunological and non-immunological factors, and post-operative management of the recipient and the graft. This is depicted schematically in Figure 1.3. 


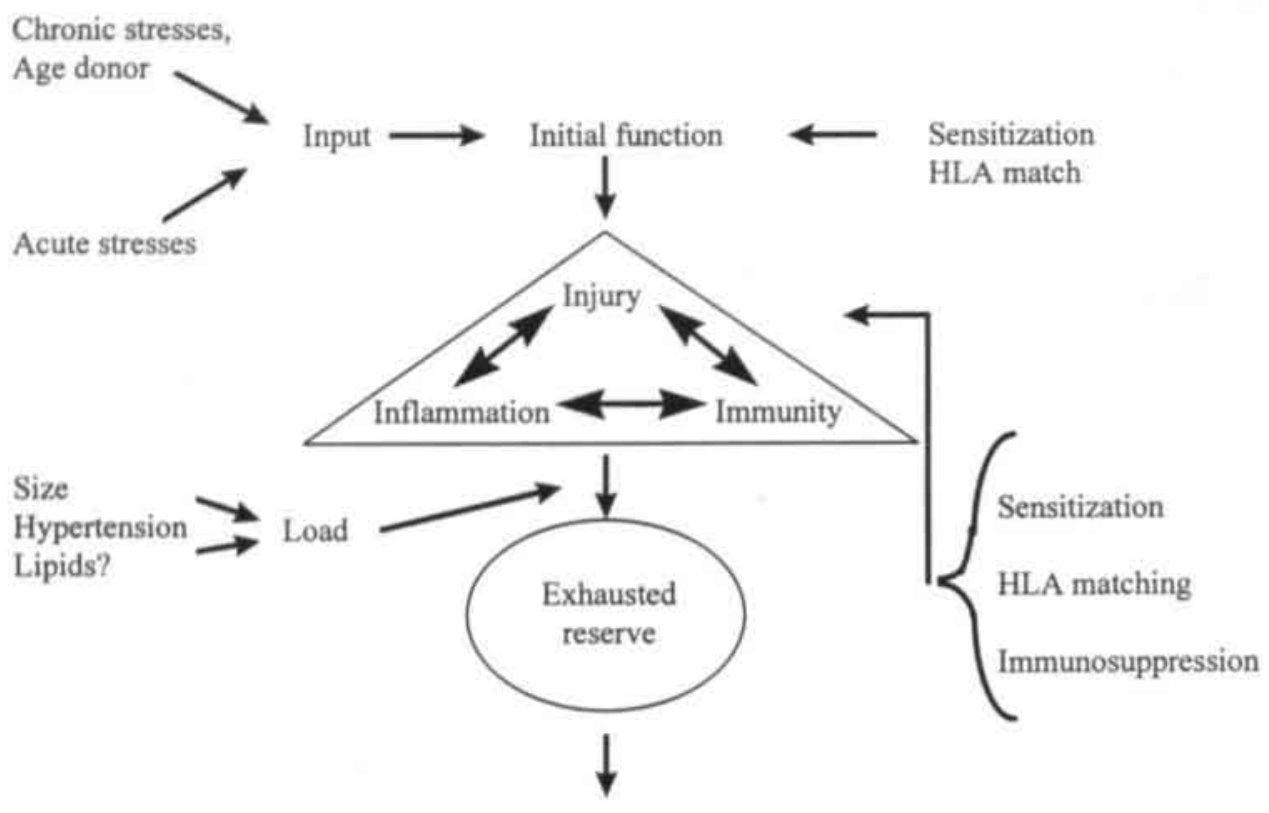

Graft failure

Figure 1.3 Interaction of input, immunity, and load factors in renal transplantation (adapted from Halloran $\mathrm{PF}^{\circ}$ ).

Many grafts are lost due to death of the recipient with a functioning graft. Also, the original disease leading to renal failure may recur leading to graft loss. Other grafts are lost due to chronic allograft nephropathy. Many non-immunological as well as immunological factors may play a role. Among the former are: aspecific injury (pre-existing and due to preservation) in the donor kidney, size mismatch of donor and recipient ${ }^{61}$, post-transplant hypertension ${ }^{62}$, non-compliance ${ }^{63}$, and possibly hyperlipidemia ${ }^{64}$. Among the immunological factors are mismatch for HLA antigens ${ }^{45,65}$ and the presence of anti-HLA antibodies ${ }^{66,67}$.

The interaction between aspecific injury and immunology on graft outcome has recently been thoroughly investigated. It has been reported that the combined presence is related to an increase in the frequency of acute rejections ${ }^{20}$ and worse graft survival ${ }^{68}$. This is supported by the facts that matching becomes more important as the ischemia time increases ${ }^{69}$, that rejection is more frequent in patients with $\mathrm{DGF}^{\circ}$, and that the combination of acute rejection and DGF results in worse graft survival ${ }^{\text {To }}$. 
Better results of living donor transplantation ${ }^{34}$ may, in part, be due to less (ischemic) injury of the graft as a result of better quality of the donor kidney and less preservation damage due to short periods of preservation. In addition, it has been reported that in hypotensive brain-dead rats, a non-immune response occurs" that may aggravate the injury to the graft. Besides the influence of kidney injury on DGF and long-term graft survival, injury-induced inflammation has been linked to the occurrence of acute rejection ${ }^{n}$.

\section{Major histocompatibility complex.}

That major histocompatibility complex (MHC; in humans "human leukocyte antigen complex") exists, has been known since the 1960s. The system consists of a number of closely linked, highly polymorphic loci. The genes of these loci code for transplantation antigens that are expressed on the cell surface. It has been shown that, in men, the MHC system is located on the short arm of chromosome $6^{73,74}$. There are two classes of MHC antigens. Class I antigens (A, B, and C) are present on nearly all nucleated cells and on platelets. MHC class II antigens (DP, DQ, and DR) were discovered in the 1970s and are present on B lymphocytes, macrophages, Langerhans and dendritic cells, activated T lymphocytes, endothelial cells, and renal tubular cells. For each of the HLA loci, many alleles have been described. Human cells are diploid and contain two homologous sets of chromosomes. Therefore, two alleles are present for each locus. The location of the HLA-A, -B, -C, and -DR on chromosome 6 in men is depicted in Figure 1.4. Table 1.2 shows the list of the serologically detected HLA antigens (A, B, and DR) that are recognized at present.

Both class I and II MHC antigens are cell surface glycoproteins. Class I antigens have a two-chain structure: a heavy glycoprotein chain with a molecular weight of about 44000 that is non-covalently linked to $\beta_{2}$-microglobulin (molecular weight about 12000). The variability accounting for different specificities resides in the heavy chain, which shows a structural homology with immunoglobulins. The heavy chain is anchored in the cell membrane by the $\mathrm{COOH}$-terminal. The remainder of the molecule is organized in three domains $\left(\alpha_{1}, \alpha_{2}\right.$, and $\left.\alpha_{3}\right)$, while the $\beta_{2^{*}}$ microglobulin forms the fourth domain. The three-dimensional structure of the class I antigen has a large groove between the $\alpha$-helices of the $\alpha_{1}$ and $\alpha_{2}$ domains. This groove provides the binding place for processed foreign antigens ${ }^{74}$. 


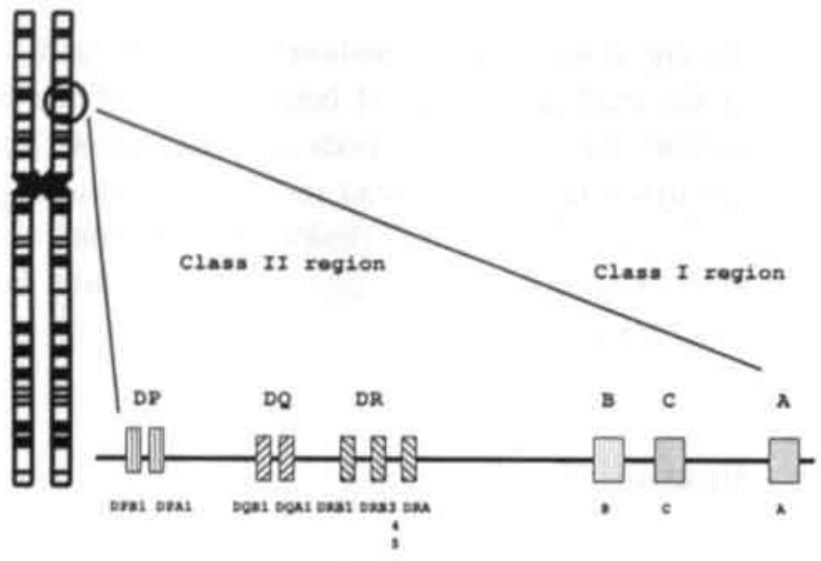

Figure 1.4 Schematic location of expressed major HLA class I and II genes on the short arm of chromosome 6 .

Table 1.2 Listing of serologically recognized HLA-A, -B, and -DR specificities ${ }^{\text {I2? }}$.

\begin{tabular}{llll}
\hline HLA-A & HLA-B & & HLA-DR \\
\hline A1 & B5 & B50(21) & DR1 \\
A2 & B7 & B51(5) & DR103 \\
A203 & B703 & B5102 & DR2 \\
A210 & B8 & B5103 & DR3 \\
A3 & B12 & B52(5) & DR4 \\
A9 & B13 & B53 & DR5 \\
A10 & B14 & B54(22) & DR6 \\
A11 & B15 & B55(22) & DR7 \\
A19 & B16 & B56(22) & DR8 \\
A23(9) & B17 & B57(17) & DR9 \\
A24(9) & B18 & B58(17) \\
A2403 & B21 & B59 & DR11(5) \\
A25(10) & B22 & B60(40) & DR12(5) \\
A26(10) & B27 & B61(40) & DR13(6) \\
A28 & B2708 & B62(15) & DR14(6) \\
A29(19) & B35 & B63(15) & DR1403 \\
A30(19) & B37 & B64(14) & DR15(2) \\
A31(19) & B38(16) & B65(14) & DR16(2) \\
A32(19) & B39(16) & B67 & DR17(3) \\
A33(19) & B3901 & B70 & DR18(3) \\
A34(10) & B3902 & B71(70) & DR51 \\
A36 & B40 & B72(70) & DR52 \\
A43 & B4005 & B73 & DR53 \\
A66(10) & B41 & B75(15) & \\
A68(28) & B42 & B76(15) & \\
A69(28) & B44(12) & B77(15) & \\
A74(19) & B45(12) & B78 & \\
A80 & B46 & B81 & \\
& B47 & Bw44 & \\
& B48 & Bw6 & \\
\hline
\end{tabular}

Antigens in parathesis 0 denote "broad" antigens from which the new specificities have "split". 
Class II antigens comprise transmembrane heterodimers, composed of two heavy chains of slightly unequal molecular weight ( $\alpha$ and $\beta$ ), non-covalently associated to each other. Both chains are anchored in the cell membrane. As with the class 1 antigen, the three-dimensional structure of the class II antigen has an antigenbinding groove.

MHC antigens are fundamental to the function of the immune response. They present self and foreign peptides, and they provide a basis for the distinction between self and non-self. Many immune responses can only occur in the context of the appropriate MHC alleles (MHC restriction). The MHC polymorphism may influence the effectiveness of the binding of peptides and thereby influence the immune response. A population with many different MHC antigens may therefore, be better equipped to survive changes in the environment. This is reflected in associations of several MHC antigens and diseases, as reviewed by Thorsby ${ }^{76}$.

\section{HLA-typing}

Typing of HLA class I antigens started in the beginning of the 1960s with an in vivo test: intradermal transfer of test lymphocytes to evoke a local graft-versus-host reaction $^{7}$. With improvements in (in vitro) serological testing, this method was soon abandoned for clinical use. The first serological test was based on agglutination. It had the disadvantage of low reproducibility, and so many different approaches were used ${ }^{7}$. Later, these tests were replaced by the complementdependent cytotoxicity $(\mathrm{CDC})$ test $^{78}$. Terasaki introduced a variant of this tests, now known as the standard NIH microlymphocytotoxicity test ${ }^{79}$.

In the 1970s, HLA class II antigens were discovered ${ }^{80}$. They could be detected by an in vitro culture of lymphocytes, the mixed lymphocyte reaction (MLR), and were named MLR-locus D. In 1975, some class II antigens could also be detected by serology and these antigens were named DR (D related) ${ }^{81}$. Because class II antigens were not present on T cells, the small B-cell population had to be enriched by T-cell depletion (e.g., by nylon wool column separation or SRBC rosetting) or by selective positive identification of B cells (two-color-fluorescence by labelling B cells with fluorescent anti-lg antibody and lysing cells stained by ethidium

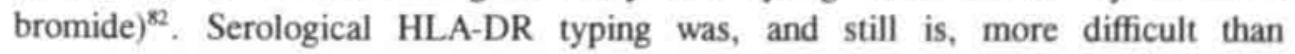
HLA-A, -B, and -C typing. This is reflected in the great percentage of discordant results for HLA-DR typing in proficiency testing.

Several HLA antigens defined in histocompatibility workshops were later recognized as representing not one antigen, but rather as consisting of different alleles. The 'group' antigen was named 'broad' antigen, while the new alleles were 
named 'splits'. Matching for 'splits' resulted, in a better outcome than matching for 'broads's3.

In the 1990s, DNA typing was introduced, showing a greater polymorphism for both HLA class I and II antigens than was revealed by serological methods. In the beginning, the restriced fragment length polymorphism (RFLP) technique was used. Due to the limitations of this method, it was soon replaced by PCR-SSOP and SSP. The SSOP method is defined as detection of HLA alleles of an individual by hybridization with Sequence Specific Oligonucleotide Probes. The method is based on fixing PCR amplified DNA to a nylon membrane and subsequent hybridization of labelled specific oligonucleotide probes with the DNA on the membrane. From the resulting hybridization pattern the HLA typing can be deduced.

With the SSP method, the HLA alleles of an individual are detected by amplification with Sequence Specific Primers (SSP). In this method, highly specific PCR reactions are used to discriminate between different HLA types. Two specific primers are allowed to amplify part of the HLA gene, and a PCR product is only formed when both primers match perfectly. Using a number of primer combinations enables the identification of the HLA alleles present.

For both PCR-SSOP and SSP, the polymorphism detected is limited to the areas where the primers and probes are located. To keep up with the increasing number of alleles discovered in recent years, the number of primers and probes have to increase equally. Therefore, Sequence-Based Typing (SBT) was introduced as high resolution typing technique in 1993. This typing strategy is based on direct DNA sequencing determining the nucleotide order of the HLA alleles present. SBT detects all polymorphisms located within the sequenced part of the gene and, therefore, enables identification of the subtypes and detection of new alleles.

That HLA differences at the nucleotide level show clinically relevance was described by Opelz et al. In recipients of a renal allograft without a serological HLA-ABDR mismatch, graft survival was lower in the group with the HLA-DR mismatch at the DNA level $(69 \%)$ than in patients without such a mismatch $(87 \%)^{84}$.

\section{Techniques for HLA typing, crossmatching, and screening for anti-HLA antibodies}

Currently, various techniques are used in the tissue typing laboratory for HLA typing, crossmatching, and to screen sera for anti-HLA antibodies.

As mentioned earlier, the complement-dependent cytotoxicity (CDC) assay is the most frequently used technique. It is based on the binding of preformed antibodies 
directed against cell surface antigens after incubation of serum and lymphocytes. In a second step, (rabbit) complement is added and activated in the presence of bound complement-fixing antibodies. As a result, the cell is lysed and the cell membrane becomes permeable to vital dye. In this way, dead cells $(=$ cells with bound complement-fixing antibodies) can be discriminated from living cells. This is schematically depicted in Figure 1.5. The CDC technique detects complementfixing antibodies (IgM and $\operatorname{IgG}$ isotypes gamma 1, 2, and 3 ) that react with cell surface antigens.

The CDC technique has many variants, the most important of which are NIH ( $\mathrm{T}$ cells, class I) and two-color fluorescence (B cells, class I+II). By combining both techniques, class I and class II reactions can be discriminated.

The ELISA technique uses selected soluble HLA antigens (sHLA) bound to a microtiter plate. Incubation with serum results in binding of antibodies directed against the HLA antigens on the plate. In a second step using anti-human IgG, IgG antibodies bound to the sHLA can be detected by an enzymatic coloring technique. This is schematically depicted in Figure 1.5.

The ELISA technique detects specific anti-HLA $\operatorname{lgG}$ (both complement-fixing and non-complement-fixing). Currently, this technique is commercially available for screening sera and is under investigation for crossmatching.

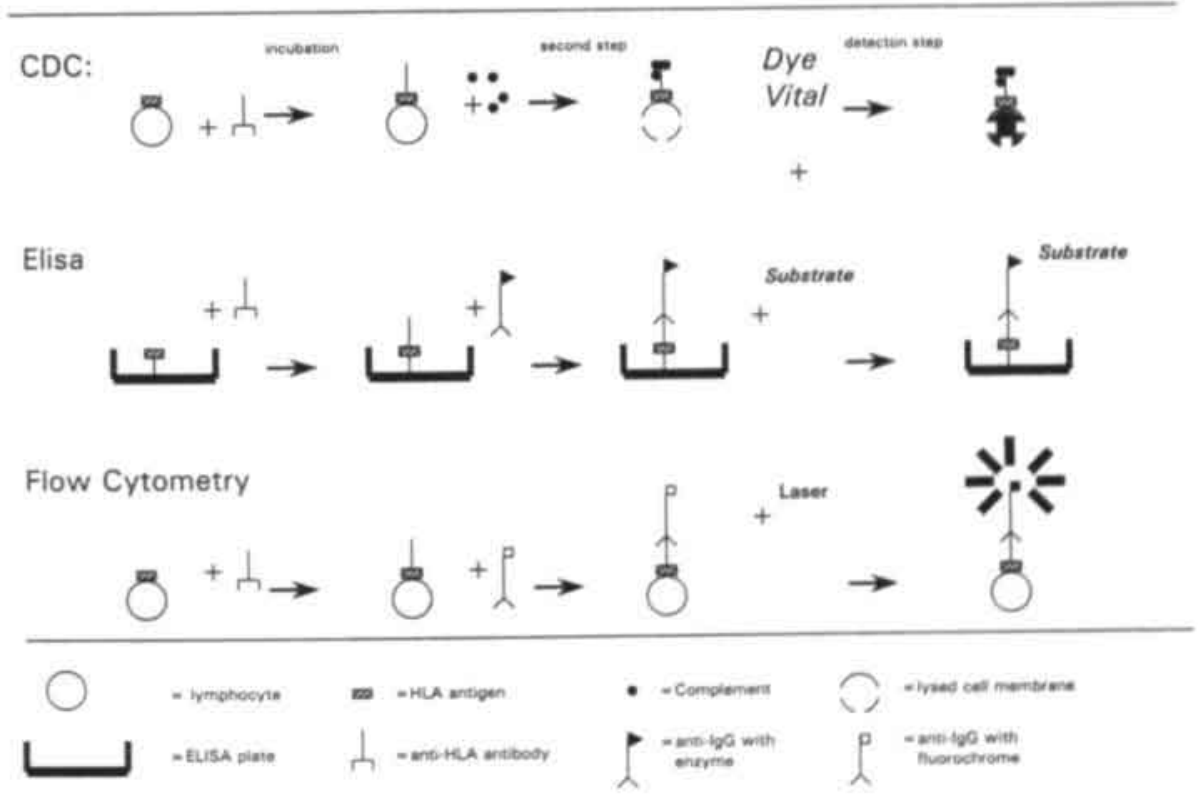

Figure 1.5 Principles of techniques for detection of anti-HLA antibodies by CDC, ELISA, and flow cytometry. 
The principle of the flow cytometry technique is described in more detail in chapter 4. Cells and serum are incubated. Cell membrane-bound antibodies are made visible by an anti-IgG antibody with a fluorescent label. This is schematically depicted in Figure 1.5. Flow cytometry detects IgG antibodies (complement-fixing and non-fixing) directed against cell surface antigens. Flow cytometry is used as a crossmatch technique, but can also be used as a first step screening test for antibody screening.

Several DNA techniques are used for HLA typing on the genomic level. Most of them are polymerase chain reaction (PCR)-based. The sequence-based typing technique is the most sophisticated and types on the level of individual nucleotides.

\section{Prevention of rejection}

Different immunological strategies for preventing rejection exist, such as HLA matching, crossmatching, screening for anti-HLA antibodies, and pretransplant transfusion.

\section{HLA matching}

The formal proof that antigens of the MHC system are important in transplantation came in the form of the excellent results of HLA-identical transplants compared to haplo-identical transplants ${ }^{85}$. Therefore, by matching donor and recipient for HLA antigens, theoretically the immune response should be less intense. While the recipient may carry antigens not present in the donor, donor antigens not present in the recipient are avoided. This matching can be performed on different levels of increasing similarity: broad, split, or DNA. The greater the similarity, the less likely an antigen will be recognized as foreign and the better the match will be. However, it is also true that the greater the level of similarity, the more difficult it will be for a recipient to receive an HLA-matched graft.

There is consensus on the beneficial effect of preventing any mismatch for HLA-A, $-B$, and -DR (zero mismatch) on graft survival ${ }^{86,87}$. Numerous reports have shown a beneficial effect of matching for less well-matched kidneys ${ }^{88.89}$. Yet, recently, the question was raised as to whether the benefit of this kind of match outweighs the detrimental effect of extended preservation time, especially when marginal donors are used ${ }^{90}$.

\section{Crossmatching}

The presence of donor-directed anti-HLA class I antibodies at the time of transplantation may induce hyperacute rejection. By performing a crossmatch, these 
antibodies can be detected pretransplantation and the transplantation can be cancelled $^{91}$. As discussed previously, the most commonly used technique is the complement-dependent cytotoxicity assay. This technique detects class I complement-fixing antibodies. In order to increase its sensitivity, several modifications have been proposed, among them modifying the incubation time or temperature ${ }^{92}$, the number of wash steps ${ }^{93}$, and adding anti-human immunoglobulin reagent ${ }^{24}$. There is no consensus as to whether these modifications result in an improved correlation with clinical outcome.

Detection of donor-directed anti-HLA antibodies after transplantation has been reported to be associated with the occurrence of acute rejection and worse graft survival $^{\text {ss }}$.

Other crossmatch techniques have been introduced. In many centers, the flow cytometry (FC) crossmatch has replaced the CDC crossmatch as the technique for pretransplant crossmatching because of its improved sensitivity and relationship to clinical outcome ${ }^{96-102}$. The principles of FC and FC crossmatch are reviewed in chapter 4. Under investigation is Crosstat, a crossmatch technique that uses soluble HLA antigens of the donor coated on ELISA plates ${ }^{103}$.

\section{Screening for anti-HLA antibodies: pre- and post-transplantation}

Anti-HLA antibodies can be formed after contact with foreign HLA antigens, such as by blood transfusion, pregnancy, and transplantation. The titer might change over time, and after each contact with HLA antigens, new specificities may be formed. Therefore, regular screening for anti-HLA antibodies of serum of patients on the renal transplant waiting list is necessary. Usually, the serum with the highest percentage of panel reactivity (peak) and the latest serum pretransplant (current) are used in the crossmatch procedure at the time of transplantation.

Preformed anti-HLA antibodies directed against mismatched donor antigens may be detrimental for graft outcome. An increase in rate of $\mathrm{DGF}^{104}$, an increase in rejection rate, and poor graft survival have been reported in the presence of antiHLA $\mathrm{IgG}^{66,67}$. Therefore, it is essential to know if a patient is sensitized because this information can be used to select the optimal donor-recipient combination or the type of immunosuppressive therapy after transplantation. Screening serum after transplantation has also been reported to be important because detection of these antibodies is reported to be related to complications and worse graft survival ${ }^{39,40}$. Studies need to be designed to answer the question of whether clinical outcome may be improved by a change in patient management after detection of these antibodies.

The presence of antibodies can be detected in screening assays. Most centers use (a variant of) the complement-dependent cytotoxicity assay. Screening results can be 
expressed as the percentage of the panel that reacts (percentage panel reactive antibodies, PRA), or as the HLA specificity detected. PRA can vary according to the composition of the panel, e.g., selected panel or random panel, and panel size. HLA specificity can be ascertained by comparing the HLA antigens of reactive lymphocytes with those of non-reactive lymphocytes. If PRA is very high ( $>85 \%)$, detection of HLA specificity may not be possible because of the low number of non-reactive lymphocytes. Positive reactions may also be caused by the presence of autoantibodies (usually of the IgM type) or non-HLA antibodies. Non-complementfixing antibodies (e.g., IgG4) are not detected even when they are anti-HLA directed. By CDC-NIH screening class 1 antibodies can be detected, while by CDC-TCF class I + II antibodies are detected. In CDC-TCF screening, reactivity to B lymphocytes is scored. Because the density of HLA class I antigens is higher on the cell membrane of B lymphocytes than that of $\mathrm{T}$ lymphocytes low avidity or low-titre HLA class I antibodies may react in the CDC-TCF and not in the CDC-NIH. To discriminate class I and class II reactions in these cases, HLA class I antibody absorption can be performed by pretreatment of serum with a pool of platelets, or cells can be treated with blocking antibodies.

Recently, other screening techniques have been introduced. Flow cytometry screening uses a small panel of cells and can be used as a quick first-line screening method to select antibody-positive sera ${ }^{105}$. Because of the large number of cells needed, it is not an ideal technique for the detection of specificity, and these sera have to be tested further by a second technique. Another technique uses soluble HLA molecules coated to an ELISA plate by anti-HLA antibodies. This technique is commercially available as Prastat (Sangstat Medical Corporation, Menlo Park, CA, USA). Its advantage is that it can detect HLA-specific IgG, both complementfixing and non-complement-fixing. Neither IgM nor autoantibodies are detected.

\section{Transfusions}

Patients with end-stage renal failure have anemia due to a lack of erythropoietin. Before the introduction of recombinant erythropoietin in the mid-1980s, patients on dialysis frequently needed blood transfusions, resulting in the production of antiHLA antibodies in $\pm 40 \%$ of all blood recipients ${ }^{106}$. This sensitization was disadvantageous, both because of the difficulty in finding a crossmatch-negative graft, and because of the negative impact of preformed antibodies on graft survival ${ }^{45}$.

In contrast, as early as 1946, Medawar showed that donor-specific blood transfusion delayed the rejection of skin homografts in rabbits ${ }^{107}$. The beneficial effect of blood transfusions in humans was shown in the mid-1960s ${ }^{108,109}$. In a multicenter study Opelz et al. showed that patients without a transfusion had worse graft survival and more rejections than transfused patients. This was called 'the 
transfusion effect ${ }^{110}$. These findings have been confirmed in a single center study $^{\text {II! }}$. It was shown that a single transfusion was sufficient to result in a benefit from 'the transfusion effect' ${ }^{\prime 11.112}$. Blood depleted of leukocytes was shown not to induce this effect ${ }^{112}$. In the early 1980s, the transfusion benefit was reported to have nearly vanished, probably due to improved results in non-transfused patients resulting from improved patient care, better matching, and the introduction of cyclosporine ${ }^{113}$. However, as recently shown by a prospective multicenter study by Opelz, also in the cyclosporine era pretransplant blood transfusion has a beneficial effect on rejection rate and graft survival in kidney allograft recipients ${ }^{114}$. The mechanisms involved in this beneficial effect are still largely unknown. Proposed hypotheses include both non-specific mechanisms, e.g., clonal deletion ${ }^{113}$, macrophage blockade by iron overload ${ }^{116}$, patient selection by production of antiHLA antibodies after transfusion, and immunological mechanisms, e.g., induction of anti-idiotypic antibodies ${ }^{117}$, and activation of suppressor cells ${ }^{118}$.

The optimal protocol to obtain the beneficial 'transfusion effect' is not known. Many factors may be of importance, e.g., the number of leukocytes ${ }^{112}$, blood storage time $e^{112,119}$ and method ${ }^{120}$, time interval between transfusion and transplantation ${ }^{121}$, patient characteristics ${ }^{121}$, and HLA compatibility between blood donor and recipient ${ }^{12 \cdot 124}$.

The sharing of at least one HLA-DR antigen between blood donor and recipient has been reported to result in less alloimmunization, a lower rejection rate, and better graft survival ${ }^{122-124}$. However, control patients in these studies generally were patients who had received transplants previously and received random, non-typed transfusions instead of typed, non-sharing transfusions. Furthermore, the number of transfusions differed between patients and controls in some studies. Also, sensitization was tested against a panel of third-party lymphocytes and was not donor-directed.

HLA-B + DR matching between blood donor and recipient resulted in a decreased frequency of cytotoxic $\mathrm{T}$ lymphocytes against mismatched class $\mathrm{I}$ antigens ${ }^{125}$. However, this study has been challenged by others ${ }^{126.127}$.

Therefore, the benefit of HLA compatibility between blood donor and recipient is still unclear.

\section{Aim of the thesis}

Screening for HLA antibodies and matching for HLA antigens have played a central role in renal allografting since the $1960 \mathrm{~s}$. However, the management of recipients, including immunosuppressives, have changed considerably and new histocompatibility techniques have been introduced. Therefore, both standard and new techniques have to be compared with each other and with clinical outcome 
parameters to evaluate their value.

The purpose of this thesis is to answer the following questions.

1. What is the incidence of donor-directed HLA antibodies after transfusion of a single unit of leukocyte-poor erythrocyte concentrate to immunologically naive patients on the renal transplant waiting list?

Is there a difference in the occurrence of these antibodies between patients receiving DR-matched blood transfusion and patients receiving nonmatched blood transfusion?

Is there a difference in acute rejection rate or graft survival after transplantation between patients receiving DR-matched blood transfusion and patients receiving non-matched blood transfusion? (Chapter 2)

2. What is the method agreement between Prastat, an ELISA-based screening technique, and NIH/TCF complement-dependent-cytotoxicity (CDC) screening? Do the two screening techniques differ regarding result of the screening and acute rejection rate or graft survival? (Chapter 3 )

3. What is the relationship between pretransplant FC crossmatch and acute rejection and 1-year renal graft survival in patients transplanted with a negative HLA class I CDC crossmatch (NIH)? (Chapter 5)

4. Do the result of FC and CDC crossmatch with post-transplant serum differ with respect to acute rejection rate or renal graft survival? (Chapter 6)

5. What is the efficacy and tolerability of tacrolimus compared to cyclosporine, what are the pharmacokinetics of tacrolimus and mycophenolic acid when used in combination, and how is tacrolimus to be used together with new drugs? (Chapter 7)

6. Which immunological and non-immunological factors influence outcome parameters in renal graft recipients on a cyclosporine-based immunosuppressive regimen transplanted at the University Hospital Maastricht? (Chapter 8) 


\section{References}

1. Kolff WJ. De kunstmatige nier. Thesis, University of Groningen, Kampen, JH Kok nv, 1946.

2. William Drukker. History of peritoneal dialysis. In: Replacement of renal function by dialysis. JF Maher (ed) 3rd edition, Kluwer Academic Publishers 1989: 487.

3. William Drukker. History of haemodialysis. In: Replacement of renal function by dialysis. JF Maher (eds) 3rd edition, Kluwer Academic Publishers 1989: 42.

4. Quinton WE, Dillard DH, Scribner BH. Cannulation of blood vessels for prolonged hemodialysis. Trans Am Soc Artif Intern Organs 1960; 6: 104.

5. Waiser J, Budde K, Schreiber M, Peibst O, Koch U, Bohler T, Hoffken B, Hauser I, Neumayer HH. The quality of life in end stage renal disease care. Transpl Int 1998: 11 Suppl 1: S42-5.

6. Schnuelle P, Lorenz D, Trede M, van der Woude FJ. Impact of renal cadaveric transplantation on survival in end-stage renal failure: evidence for reduced mortality risk compared with hemodialysis during long-term follow-up. J Am Soc Nephrol 1998; 9: 2135-41.

7. Karlberg I, Nyberg G. Cost-effectiveness studies of renal transplantation. Int J Technol Assess Health Care 1995; 11: 611-22.

8. Murray JE, Merrill JP, Harrison JH. Renal homotransplantation in identical twins. Surg Forum 1955; 6: 432-3.

9. Starzl TE, Marchioro TL, Holmes JH, Hermann G, Brittain RS, Stonington OH, Talmage DW, Waddell WR. Renal homografts in patients with major donorrecipient blood group incompatibilities. Surgery 1964; 55: 195-200.

10. Dausset J, Nenna A. Presence d'une leuco-agglutinine dans le serum d'un cas d'agranulocytose chronique. Compt. Rend. Soc. Biol.(Paris) 1952; 146: 1539-41.

11. Dausset J. Iso-leuco-anticorps. Acta Haematol. 1958; 20: 156-66.

12. Payne $R$. The association of febrile transfusion reactions with leuko-agglutinins. Vox Sang. 1957; 2: 233-41.

13. van Rood JJ, van Leeuwen A, Eernisse JG. Leucocyte antibodies in sera of pregnant women. Vox Sang. 1959; 4: 427-44.

14. van Rood JJ, Eernisse JG, van Leeuwen A. Leucocyte antibodies in sera from pregnant women. Nature 1958; 181: 1735-6.

15. van Rood JJ. A proposal for international cooperation in organ transplantation, Eurotransplant. Histocompatibility Testing. Munksgaard, Copenhagen 1967: 451.

16. Worldwide transplant center directory kidney transplants. In: Cecka JM, Terasaki PI (eds). Clinical Transplants 1998, Los Angeles, UCLA Tissue Typing Laboratory. 1999: 519.

17. Statistisch verslag van de Registratie Nierfunctievervanging Nederland (Renine) 1998. Eds Ramsteijn PG, de Charro FTh, Geerlings W, Krediet RT, Tjandra YI. Utrecht, Elinkwijk 1998: 22.

18. Paller MS. Free radical-mediated postischemic injury in renal transplantation. Renal Failure 1992; 14: 257-60. 
19. Kahan BD, Mickey R, Flechner SM, Lorber MI, Wideman CA, Kerman RH, Terasaki PI, van Buren CT. Multivariate analysis of risk factors impacting on immediate and eventual cadaver allograft survival in cyclosporine-treated recipients. Transplantation 1987; 43: 65-70.

20. Halloran P, Aprile M, Farewell V, Ontario renal transplant research group. Factors influencing early renal function in cadaver kidney transplants. A case-control study. Transplantation 1988; 45: 122-7.

21. Ploeg RJ, van Bockel JH, Langendijk PTH, Groenewegen $\mathrm{M}$, van der Woude FJ, Persijn GG, Thorogood J, Hermans J. Effect of preservation solution on results of cadaveric kidney transplantation. Lancet 1992; 340: 129-36.

22. Luciani J, Frantz P, Thibault P, Ghesquierre F, Conseiller C, Cousin MT, Glaser P, LeGrain M, Viars P, Kuss R. Early anuria prevention in human kidney transplantation. Advantage of fluid load under pulmonary arterial pressure monitoring during surgical period. Transplantation 1979; 28: 308-12.

23. Iwaki Y, Terasaki PI. Primary nonfunction in human cadaver kidney transplantation: Evidence for hidden hyperacute rejection. Clin Transplant 1987; 1: 125-31.

24. Pratschke J, Wilhelm MJ, Kusaka M, Basker M, Cooper DKC, Hancock WW, Tilney NL. Brain death and its influence on donor organ quality and outcome after transplantation. Transplantation 1999; 67: 343-8.

25. Rijksen JFWB. Composition of the Euro-Collins solution. Eurotransplant manual 1972, Leiden, the Netherlands 1972: 30

26. Wahlberg JA, Love R, Landegaard L, Southhard JH, Belzer FO. 72-hour preservation of the canine pancreas. Transplantation 1987; 43: 5-8.

27. Isemer FE, Ludwig A, Schunk O, Bretschneider HJ, Peiper HJ. Kidney organ procurement with HTK solution of Bretschneider. Transplant Proc. 1988; 20: 885-6.

28. Groenewoud AF, Thorogood J. Current status of the Eurotransplant randomized multicenter study comparing kidney graft preservation with histidine-tryptophanketoglutarate, university of Wisconsin, and Euro-Collins solutions. The HTK study group. Transplant Proc 1993; 25 (1 pt 2): 1582-5.

29. Booster MH, Wijnen RMH, Yin M, Tiebosch ATM, Heineman E, Maessen JG, Buurman WA, Kurvers HAJM, Stubenitsky BM, Bonke H, Kootstra G. Enhanced resistance to the effects of normothermic ischemia in kidneys using pulsatile machine perfusion. Transplant Proc 1993; 25: 3006-11.

30. Cecka JM, Terasaki PI. The UNOS scientific renal transplant registry-1991. In: Terasaki PI, Cecka JM (eds). Clinical Transplants 1991, Los Angeles, UCLA Tissue Typing Laboratory. 1992: 1-11.

31. Leunissen KML, Bosman FT, Nieman FHM, Kootstra G, Vromen MAM, Noordzij TC, van Hooff JP. Amplification of the nephrotoxic effect of cyclosporine by preexistent chronic histological lesions in the kidney. Transplantation 1989; 48: 590-3. 
32. Wijnen RMH, Booster MH, Stubenitsky BM, de Boer J, Heineman E, Kootstra G. Outcome of transplantation of non-heart-beating donor kidneys. Lancet 1995; 345; $1067-70$.

33. Cho YW, Terasaki PI. Cecka JM, Gjertson DW. Transplantation of kidneys from donors whose hearts have stopped beating. NEJM 1998; 338: 221-5.

34. Terasaki PI, Cecka JM, Gjertson DW, Takemoto S. High survival rates of kidney transplants from spousal and living unrelated donors. N Engl J Med 1995; 333: 333-6.

35. van Hooff JP, van Es A, Koolen MI, Kalff MW, van den Broek PH, de Graeff J. Less aggressive rejections treatment and low-dose corticosteroids leading to satisfactory cadaveric kidney graft survival and low morbidity rate. Proc EDTA 1980: 17: 435-9.

36. Liu J, Farmer JD, Lane WS, Friedman J, Weissman I, Schreiber SL. Calcineurin is a common target of cyclophilin-cyclosporine A and FKBP-FK506 complexes. Cell 1991; 66: 807-15.

37. Racusen LC, Solez K, Colvin RB, Bonsib SM, Castro MC, Cavallo T, Croker BP, Demetris AJ, Drachenberg CB, Fogo AB, Furness P, Gaber LW, Gibson IW, Glotz D, Goldberg JC, Grande J, Halloran PF, Hansen HE, Hartley B, Hayry PJ, Hill CM, Hoffman EO, Hunsicker LG, Lindblad AS, Marcussen N, Mihatsch MJ, Nadasdy T, Nickerson P, Olsen TS, Papadimitriou JC, Randhawa PS, Rayner DC, Roberts I, Rose S, Rush D, Salinas-Madrigal L, Salomon DR, Sund S, Taskinen E, Trpkov K, Yamagushi Y. The Banff 97 working classification of renal allograft pathology. Kidney Int. 1999; 55: 713-23.

38. Slakey DP. Thomas JM. Mechanism of cell-mediated rejection. In: Kidney transplant rejection. 3rd edition, revised and expanded. Eds: Racusen LC, Solez K, Burdick JF. New York, Marcel Dekker Inc. 1998: 33.

39. Martin S, Dyer PA, Mallick NP, Gokal R, Johnson RW. Posttransplant anti-donor lymphocytotoxic antibody production in relation to graft outcome. Transplantation 1987: 44: 50-3.

40. Lobo PI, Spencer CE, Stevenson WC, Pruett TL. Evidence demonstrating poor kidney graft survival when acute rejections are associated with $\mathrm{IgG}$ donor-specific lymphocytotoxin. Transplantation 1995: 59: 357-60.

41. Slakey DP. Thomas JM. Mechanism of cell-mediated rejection. In: Kidney transplant rejection. Eds: Racusen LC, Solez K, Burdick JF. 3rd edition, revised and expanded. New York, Marcel Dekker Inc. 1998: 49.

42. Tullius SG, Hancock WW, Heemann U, Azuma H, Tilney NL. Reversibility of chronic renal allograft rejection. Critical effect of time after transplantation suggests both host immune dependent and independent phases of progressive injury. Transplantation 1994; 58: $93-9$.

43. Hoitsma AJ, van Lier HJ, Reekers P, Koene RA. Improved patient and graft survival after treatment of acute rejections of cadaveric renal allografts with rabbit antithymocyte globulin. Transplantation 1985; 39: 274-9.

44. Schwartz RS, Dameshek W. Drug induced immunological tolerance. Nature 1959; 183: $1682-3$. 
45. van Hooff JP, van der Steen GJ, Schippers HMA, van Rood JJ. Efficacy of HL-A matching in Eurotransplant. Lancet 1972; 2: 1385-8.

46. van Es A, Persijn GG, van Hooff-Eijkenboom YEA, Kalff MW, van Hooff JP. Blood transfusions, HLA-A and B, DR matching, graft survival, and clinical course after cadaveric kidney transplantation. Transplant Proc 1981; 13: 172-4.

47. Starzl TE, Marchiori TL, Porter KA, Iwasaki Y, Cerelli CJ. The use of heterologous antilymphoid agents in canine renal and liver homotransplantation, and in human renal homotransplantation. Surgery Gynaecology, and Obstetrics 1967; 124: 301-18.

48. Calne RY, Rolles K, White DJ, Thiru S, Evans DC, McMaster P, Dunn DC, Craddock GN, Henderson RG, Aziz S, Lewis P. Cyclosporine A initially as the only immunosuppressant in 34 recipients of cadaveric organs. Lancet 1979; ii: 1033-36.

49. Schreiber S, Crabtree G. The mechanism of action of cyclosporine A and FK506. Immunol Today 1992; 12: 136-42.

50. Hall BM, Tiller DJ, Hardie I, Mahony J, Mathew T, Thatcher G, Miach P, Thomson N, Sheil AG. Comparison of three immunosuppressive regimens in cadaver renal transplantation: long-term cyclosporine, short term cyclosporine followed by azathioprine and prednisolone, and azathioprine and prednisolone without cyclosporine. N Eng J Med 1988; 318: 1499-507.

51. European Multicentre Trial Group. Cyclosporine in cadaveric renal transplantation: one year follow-up of a multicentre trial. Lancet 1983; 2: 986-9.

52. The Canadian Multicentre Transplant Study Group. A randomized clinical trial of cyclosporine in cadaveric renal transplantation. N Engl J Med 1983; 309: 809-15.

53. Friman S, Bäckman L. A new microemulsion formulation of cyclosporine; pharmacokinetic and clinical features. Clin Pharmacokinet 1996; 30: 181-93.

54. Mayer AD, Dmitrewski J, Squifflet JP, Bessen T, Grabensee B, Klein B, Eigler FW, Heemann U, Pichlmayr R, Behrend M, Vanrenterghem Y, Donck J, van Hooff J, Christiaans M, Morales JM, Andres A, Johnson RWG, Short C, Buchholz B, Rehmert N, Land W, Schleibner S, Forsythe JLR, Talbot D, Neumayer HH, Hauser I, Ericzon BG, Brattström C, Claesson K, Mühlbacher F, Pohanka E. Multicenter randomized trial comparing tacrolimus (FK506) and cyclosporine in the prevention of renal allograft rejection. Transplantation 1997: 64: 436-43.

55. Pirsch JD, Miller J, Deierhoi MH, Vincenti F, Filo RS. A comparison of tacrolimus (FK506) and cyclosporine for immunosuppression after cadaveric renal transplantation. FK506 Kidney transplant study group. Transplantation 1997; 63: 977-83.

56. Christiaans M, van Hooff J, van Duijnhoven E. Single center experience of tacrolimus versus cyclosporine-neoral in renal transplantation. Kidney Int 1999; 55: 2598.

57. U.S. Renal Transplant Mycophenolate Mofetil Study Group. Mycophenolate mofetil for the prevention of acute rejection in primary cadaveric renal allograft recipients. Transplantation 1995; 60: 225-32. 
58. European Mycophenolate Mofetil Cooperative Study Group. Placebo-controlled study of mycophenolate mofetil combined with cyclosporine and corticosteroids for prevention of acute rejection. Lancet 1995; 345: 1321-5.

59. van Hooff JP, Christiaans MHL. Use of tacrolimus in renal transplantation. Transplant Proc 1999 in press.

60. Halloran PF. Transplant Proc 1998; 30: S27.

61. Lee LS, Auersvald LA, Claus EB, Bia MJ, Friedman AL, Lorber MI, Basadonna GP. Body size mismatch between donor and recipient and the development of chronic rejection in renal transplantation. Transplant Proc 1997; 29: 111.

62. Opelz G, Wujciak T, Ritz E. Association of chronic kidney graft failure with recipient blood pressure. Kidney Int 1998; 53: 217-22.

63. Hong JH. Sumrani N, Delanay V, Davis R, Dibenedetto A, Butt KM. Causes of late renal allograft failure in the ciclosporin era. Nephron 1992; 62: 272-9.

64. Kobashigawa JA, Kasiske BL. Hyperlipidemia in solid organ transplantation. Transplantation 1997; 63: 331-8.

65. Thorogood J, Persijn GG, Schreuder GMTh, D'Amaro J, Zantvoort FA, van Houwelingen JC, van Rood JJ. The effect of HLA matching on kidney graft survival in separate posttransplantation intervals. Transplantation 1990; 50; 146-50.

66. Chapman JR, Taylor CJ, Ting A, Morris PJ. Immunoglobulin class and specificity of antibodies causing positive $\mathrm{T}$ cell crossmatches. Relationship to renal transplant outcome. Transplantation 1986; 42: 608-13.

67. Taylor CJ, Chapman JR, Ting A, Morris PJ. Characterization of lymphocytotoxic antibodies causing a positive crossmatch in renal transplantation. Transplantation 1989; 48: 953-8.

68. Yilmaz S, Paavonen T, Häyry P. Chronic rejection of rat renal allografts. Transplantation 1992; 53: 823-7.

69. van Es A, Hermans J, van Bockel JH, Persijn GG, van Hooff JP, de Graeff J. Effect of warm ischemia time and HLA (A and B) matching on renal cadaveric graft survival and rejection episodes. Transplantation 1983; 36: 255-8.

70. Shoskes DA, Cecka JM. Deleterious effects of delayed graft function in cadaveric renal transplant recipients independent of acute rejections. Transplantation 1998; 66: 1697-701.

71. van der Hoeven JAB, Ploeg RJ, Postema F, Molema G, de Vos P, Girbes ARJ, van Suylichem PTR, Ter Horst GJ. Induction of organ dysfunction and activation of inflammatory markers in donor liver and kidney during hypotensive brain death. Transplant Proc. 1999; 31: 1006-7.

72. Halloran PF. Non-immunologic tissue injury and stress in chronic allograft dysfunction. Graft 1998; 1: 25-9.

73. van Someren H, Westerveld A, Hagemeijer A, Mees JR, Meera Khan P, Zaalberg OP. Human antigen and enzyme markers in man-Chinese hamster somatic cell hybrids: Evidence for synteny between HL-A, PGM3, ME1, and IPO-B loci. Proc Natl Acad Sci USA 1974; 71: 962-5. 
74. Breuning MH, van den Berg-Loonen PM, Bernini LF, Bijlsma JB, van Loghem E, Meera Khan P, Nijenhuis LE. Localization of HLA on the short arm of chromosome 6. Hum Genet 1977; 37: 131-9.

75. Bjorkman PJ, Saper MA, Samraoui B, Bennett WS, Strominger J, Wiley DC. The foreign antigen binding site and $\mathrm{T}$ cell antigen recognition regions of class $\mathrm{I}$ histocompatibility antigens. Nature 1987; 329: 512-28.

76. Thorsby E. Invited anniversary review: HLA associated diseases. Human Immunology 1997: 53: 1-11.

77. van Rood JJ. Leucocyte grouping. A method and its application. Thesis Leiden. 1962 Pasmans Den Haag.

78. Engelfriet CP, Brittan A. The cytotoxic test for leucocyte antibodies: a simple and reliable technique. Vox Sang 1965; 10: 660-74.

79. Terasaki PI, McClelland JD. Microdroplet assay of human serum cytotoxins. Nature 1964; 204: 998-1000.

80. Eijsvoogel VP, van Rood JJ, Du Toit ED, Schellekens PT. Position of a locus determining mixed lymphocyte reaction distinct from the known HL-A loci. Eur. J. Immunol. 1972; 2: 413-8.

81. van Rood JJ, van Leeuwen A, Keuning JJ, van Oud Alblas AB. The serological recognition of the human MLC determinants using a modified cytotoxicity technique. Tissue Antigens 1975; 5: 73-9.

82. van Rood JJ, van Leeuwen A, Ploem JS. Simultaneous detection of two cell populations by two-colour fluorescence and application to the recognition of B cell determinants. Nature 1976; 262: 795-7.

83. Opelz G. Importance of HLA antigen splits for kidney transplant matching. Lancet 1988; 2: 61-4.

84. Opelz G, Mytilineos J, Scherer S, Dunckley H, Trejaut J, Chapman J, Middleton D. Savage D. Fischer O, Bignon JD, et al. Survival of DNA HLA-DR typed and matched renal cadaver transplants. The Collaborative Transplant Study. Lancet 1991; 383: 461-3.

85. Singal DP, Mickey MR, Terasaki PI. Serotyping for homotransplantation. 23. Analysis of kidney transplants from parental versus sibling donors. Transplantation 1969: 7: 246-58.

86. Hata Y, Cecka JM, Takemoto S, Ozawa M, Cho YW, Terasaki PI. Effects of changes in the criteria for nationally shared kidney transplants for HLA-matched patients. Transplantation 1998; 65: 208-12.

87. Persijn GG. D'Amaro J, de Lange P, Schreuder GMTh, Thorogood J, Zantvoort F, van Rood JJ. The effect of mismatching and sharing of HLA-A, -B, and -DR antigens on kidney graft survival in Eurotransplant 1982- 1988. In: Terasaki PI (ed). Clinical Transplants 1988, Los Angeles, UCLA Tissue Typing Laboratory. 1989: 237-48.

88. Persijn GG, Gabb BW, van Leeuwen A, Nachtegaal A, Hoogeboom J, van Rood JJ. Matching for HLA antigens of A, B and DR loci in renal transplantation by Eurotransplant. Lancet 1978; i: 1278-81. 
89. Cecka JM. The UNOS scientific renal transplant registry. In: Cecka JM, Terasaki PI (eds). Clinical Transplants 1998, Los Angeles, UCLA Tissue Typing Laboratory. 1999: 1-16.

90. Fuggle $\mathbf{S}$ on behalf of Members of the Kidney Advisory Group, UKTSSA. The effect of HLA-A, B, DR mismatches on kidney transplant survival in the UK. Poster XVII World Congress of the Transplantation Society, Montreal, July 12-17 1998.

91. Patel R, Terasaki PI. Significance of the positive crossmatch test in kidney transplantation. N Engl J Med 1969; 280: 735-9.

92. Iwaki Y, Terasaki PI, Park MS, Heintz R, Silberman H, Berne T. Dilutions and specificity analysis of pretransplant sera. Transplant Proc 1979; 11: 944-9.

93. Amos DB, Bashir H, Boyle W. MacQueen M, Tülikainen A. A simple microcytotoxicity test. Transplantation 1969; 7: 220-3.

94. Johnson AH, Rossen RD, Butler WT. Detection of alloantibodies using a sensitive antiglobulin microcytotoxicity test: identification of low levels of preformed antibodies in accelerated allograft rejection. Tissue Antigens 1972; 2: 215-26.

95. Trpkov K, Campbell P, Pazderka F, Cockfield S, Solez K, Halloran PF. Pathologic features of acute renal allograft rejection associated with donor-specific antibody. Transplantation 1996; 61: 1586-92.

96. Garovoy MR, Rheinschmidt MA, Bigos M, Perkins H, Colombe B, Feduska N, Salvatierra O. Flow Cytometry Analysis: A high technology crossmatch technique facilitating transplantation. Transplant Proc 1983; 15: 1939-44.

97. Chapman JR, Deierhoi MH, Carter NP, Ting A, Morris PJ. Analysis of Flow cytometry and cytotoxicity crossmatches in renal transplantation. Transplant Proc 1985; 17: 2480-1.

98. Talbot D, Givan AL, Shenton BK, Stratton A, Proud G, Taylor RMR. Rapid detection of low levels of donor specific IgG by flow cytometry with single and dual colour fluorescence in renal transplantation. J. of Immunological Methods 1988; 112: $279-83$.

99. Cook DJ, Terasaki PI, Iwaki Y, Terashita GY, Lau M. An approach to reducing early kidney transplant failure by flow cytometry crossmatching. Clin Transplantation 1987: 1: 253-6.

100. Cook DJ, Terasaki PI, Iwaki Y, Terashita G, Fujikawa J, Gera J, Takeda A, Danovitch G, Rosenthal JT, Fine R, Ettenger R, Mendez R, Dainko E, Kavalich A, Martin D, Teichman S, Ward H, Koyle M, Berne T, Brennan LP, Lieberman E, Cohen A. Strauss F. Flow cytometry crossmatching for kidney transplantation. In: Terasaki PI (ed). Clinical Transplants 1988, Los Angeles, UCLA Tissue Typing Laboratory. 1989: 375-80.

101. Kerman RH, Van Buren CT, Lewis RM, DeVera V, Baghdahsarian V, Gerolami $\mathrm{K}$, Kahan BD. Improved graft survival for flow cytometry and antihuman-globulin crossmatch-negative retransplant recipients. Transplantation 1990; 49: 52-6.

102. Talbot D, Givan AL, Shenton BK, Stratton A, Proud G, Taylor RMR. The relevance of a more sensitive crossmatch assay to renal transplantation. Transplantation 1989; 47: 552-5. 
103. Buelow R, Chiang TR, Monteiro F, Cornejo MC, Ellingson L, Claas F, Gaber O, Gelder F, Kotb M, Orosz C, Pouletty P. Soluble HLA antigens and ELISA - a new technology for crossmatch testing. Transplantation 1995; 60: 1594-9.

104 Baldwin WM, Halloran PF. Clinical syndromes associated with antibody in allografts. In: Kidney transplant rejection. 3rd edition, revised and expanded. Eds: Racusen LC, Solez K, Burdick JF. New York, Marcel Dekker Inc. 1998: 134.

105. Harmer AW, Sutton M, Baune A, Vaughan RW, Welsh KI. A highly sensitive, rapid screening method for the detection of antibodies directed against HLA class I and II antigens. Transpl Int 1993; 6: 277-80.

106. Opelz G, Graver B, Mickey MR, Terasaki PI. Lymphocytotoxic antibody response to transfusions in potential kidney transplant recipients. Transplantation 1981; 32: 177-83.

107. Medawar PB. Immunity to homologous grafted skin. II. The relationship between the antigens of blood and skin. Brit J Exp Pathol 1946; 27: 15-24.

108. Dossetor JB, Mackinnon K, Gault M, Maclean LD. Cadaver kidney transplants. Transplantation 1967; 5: 844-53.

109. Michielsen P. Hemodialyse et transplantation renale. Proc EDTA 1966; 3: 162-7.

110. Opelz G, Sengar DPS, Mickey MR, Terasaki PI. Effect of blood transfusions on subsequent kidney transplants. Transplant Proc 1973; 5: 253-9.

111. van Hooff JP, Kalff MW, Poelgeest AE, Persijn GG, van Rood JJ. Blood transfusions and kidney transplantation. Transplantation 1976; 22: 306-7.

112. Persijn GG, Cohen B, Lansbergen Q, van Rood JJ. Retrospective and prospective studies on the effect of blood transfusions in renal transplantation in the Netherlands. Transplantation 1979; $28: 396-401$.

113. Opelz G. Improved kidney graft survival in nontransfused recipients. Transplant Proc 1987; 19: 149-52.

114. Opelz G, Vanrenterghem Y, Kirste G, Gray DWR, Horsburgh T, Lachance JG, Largiader F, Lange H, Vujaklija-Stipanovic K, Alvarez-Grande J, Schott W, Hoyer J, Schnuelle P, Descoeudres C, Ruder H, Wujciak T, Schwarz V. Prospective evaluation of pretransplant blood transfusions in cadaver kidney recipients. Transplantation 1997; 63: 964-7.

115. Terasaki PR. The beneficial transfusion effect on kidney graft survival attributed to clonal deletion. Transplantation 1984; 37: 119-25.

116. Keown PA, Descamps B. Improved renal allograft survival after blood transfusion: a non-specific, erythrocyte mediated immunoregulatory process? Lancet 1979; 1: 20-2.

117. Singal DP, Joseph S. Role of blood transfusions in the induction of antibodies against recognition sites on $\mathrm{T}$ lymphocytes in renal transplant patients. Human Immunol 1982; 4: 93-108.

118. Lagaaij EL, Ruigrok M, van Rood JJ, Hendriks GF, van der Woude F, Weimar W, van Houwelingen $\mathrm{HC}$, Goulmy E. Blood transfusion induced changes in cellmediated lympholysis: to immunize or not to immunize. J Immunol 1991; 147: 3348-52. 
119. Mincheff MS, Meryman HT, Kapoor V, Alsop P, Wotzel M. Blood transfusion and immunomodulation: a possible mechanism. Vox Sang 1993; 65: 18-23.

120. Opelz G, Terasaki PI. Poor kidney transplant survival in recipients with frozen blood transfusions or no transfusions. Lancet 1974; $2: 696-8$.

121. Cecka JM, Cicciarelli J. The transfusion effect. In: Terasaki PI (ed). Clinical Transplants 1986, Los Angeles, UCLA Tissue Typing Laboratory. 1987: 73.

122. Lagaaij EL, Hennemann IPH, Ruigrok M, de Haan MW, Persijn GG, Termijtelen A, Hendricks GF, Wiemar W, Claas FH, van Rood J. Effect of one-HLA-DRantigen-matched and completely HLA-DR-mismatched blood transfusions on survival of heart and kidney allografts. N Eng J Med 1989; 321 (11): 701-5.

123. Middleton D, Martin J, Douglas J, McClelland M. Transfusion of one HLA-DR antigen-matched blood to potential recipients of a renal allograft. Transplantation 1994; 58 (7): 845-8.

124. Bayle F, Masson D, Zaoui P, Vialtel P, Janbon B, Bensa J-C, Cordonnier DJ. Beneficial effects of one HLA haplo- or semi-identical transfusion versus three untyped blood units on alloimmunization and acute rejection episodes in first renal allograft recipients. Transplantation 1995; 59: 719-23.

125. van Twuyver E, Mooijaart RJ, ten Berge IJ, van der Horst AR, Wilmink JM, Kast WM, Melief CJ, de Waal LP. Pretransplant blood transfusion revisited. N Engl J Med 1991; 325: 1210-3.

126. Young NT, Roelen DT, Iggo N. Gray DW, Roake JA, Graham V, Wood KJ, Dallman MJ, Welsh KI, Morris PJ. Effect of one-HLA-haplotype-matched and HLA-mismatched blood transfusions on recipient T lymphocyte allorepertoires. Transplantation 1997; 63: 1160-5.

127. Baudouin V, De Vitry N, Hiesse C, Lang P, Bloch J, Legouvello S, Sterkers G. Cytotoxic $\mathrm{T}$ lymphocyte changes after HLA-DR match and mismatch blood transfusion. Transplantation 1997; 63: 1155-60.

128. Bodmer JG, Marsh SGE, Albert ED, Bodmer WF, Bontrop RE, Charron D, Dupont B, Erlich HA, Fauchet R, Mach B, Mayr WR, Parham P, Sasazuki T, Schreuder GMTh, Strominger JL, Svejgaard A, Terasaki PI. Nomenclature for factors of the HLA system, 1996. Tissue Antigens 1997; 49: 293-321. 


\section{Chapter 2}

\section{HLA-DR matched transfusions. Development of donor-specific T- and B-cell antibodies and renal allograft outcome}

MHL Christiaans, JP van Hooff, F Nieman, EM van den Berg-Loonen

Transplantation 1999; 67: 1029-35 


\section{Abstract}

\section{Background}

Pretransplant blood transfusions are reported to decrease acute rejection rate and increase graft survival after renal transplantation. This has been attributed to matching for HLA-DR with the transfusion donor, which also results in a lower rate of sensitization.

\section{Methods}

The development of donor-specific T- and B-cell antibodies was measured by National Institutes of Health and two-color fluorescence assays after one transfusion in 247 naive patients. Auto-crossmatches were performed to exclude autoantibodies. Patients were grouped according to DR matching $(n=107)$ or nonmatching $(\mathrm{n}=140)$ with the transfusion donor. In 103 renal allograft recipients, acute rejection rate and graft survival were analyzed by Cox regression.

\section{Results}

T-cell antibodies developed in $6.5 \%$ of the patients. There was no difference between the DR-matched and nonmatched group. No auto-antibodies against T-cells developed, whereas one quarter of the sera had a positive B-cell auto-crossmatch. There was no difference with regard to B-cell antibodies (auto-antibody-positive sera excluded) between the DR-matched $(15.8 \%)$ and non-matched $(18.6 \%)$ group. Sharing of HLA A and/or B antigens did not result in a lower frequency of donordirected T- or B-cell antibodies. None of the risk factors, including DR sharing with transfusion donor, contributed significantly towards graft survival (odds ratio for DR sharing: $1.02 ; 95 \% \mathrm{Cl}: 0.45-2.32 ; P=0.97)$. DR sharing was no risk factor towards acute rejection either, in contrast to DR mismatch with kidney donor (odds ratio: 2.9 ), and use of cyclosporine versus tacrolimus (odds ratio: 4.4).

\section{Conclusions}

Development of donor-directed T-cell antibodies after one transfusion of leukocytepoor blood is low and irrespective of HLA-DR match with transfusion donor. B-cell antibodies develop more frequently and independent of HLA-DR match. In $26 \%$ of the sera, B-cell auto-antibodies are detected. Rejection rate and graft survival are not significantly different between HLA-DR-matched and nonmatched transfusions. 


\section{Introduction}

In both the azathioprine and the cyclosporine (CsA) era, the beneficial effect of pretransplant blood transfusion has been proven on rejection rate and graft survival in kidney allograft recipients ${ }^{1,2}$. To obtain the beneficial effect different transfusion protocols are used. The minimal quantity and type of transfusion are still under debate. The transfusion effect was already demonstrable after a single transfusion of packed erythrocytes ${ }^{3.4}$, whereas neither filtrated leukocyte-poor erythrocytes ${ }^{4}$ nor frozen blood ${ }^{5.6}$ seemed to induce the desired effect.

A drawback of transfusion is the possible induction of anti-HLA antibodies. Development of HLA antibodies makes it more difficult to find a suitable donor organ and counterbalances the beneficial effect of transfusion on rejection rate and graft survival. Sensitization has been reported to be less frequent if transfusion donor and recipient share certain HLA-antigens; this has been reported for sharing of one HLA-DR allele ${ }^{7,8}$ or a complete haplotype ${ }^{9}$. In these studies, sensitization was determined by screening the recipient sera for cytotoxic antibodies against a panel of third-party lymphocytes. Sera were not tested against transfusion donor lymphocytes, nor were the antibodies differentiated in B-cell and T-cell directed antibodies. Only in the prospective study of Lagaaij was the presence of autoantibodies taken into account.

Sharing of an HLA-DR antigen or a complete haplotype between transfusion donor and recipient has been reported to result in fewer rejection episodes after renal transplantation $^{8.9}$ and a better renal graft survival'. The majority of control patients used in these studies were patients who had received transplants previously and received random nontyped blood transfusions instead of typed nonsharing transfusions ${ }^{8.9}$. Changes in patient management over the years may well influence outcome parameters; therefore, the real effect of sharing or nonsharing for HLA-DR is not clear. The studies were usually small in size ${ }^{7,8}$, and one of them was a multicenter study?

The frequency of occurrence of anti-HLA antibodies after a single random transfusion in recipients previously not challenged with non-self-HLA antigens is not well known. In particular, data about the specificity of these antibodies are lacking as well as data on their being donor-directed.

In this study, we investigated the influence of transfusing a single unit of either DR-matched or nonmatched blood on the development of donor-directed anti-HLA antibodies in a large cohort of immunologically naive patients before renal transplantation. Rejection rate and graft survival were compared between patients 
receiving only 1 unit of either HLA-DR-matched or nonmatched blood before transplantation.

\section{Patients and Methods}

\section{Transfusion protocol}

Leukocyte-poor blood was contracted from the local blood banks. Until 1988, the center transfusion policy consisted of administration of three blood transfusions with an interval of 1 month between transfusions to all patients on the waiting list for transplantation. Patients entering the protocol had never been transfused before or, for female patients, were not immunized by pregnancy. If a patient became immunized after one of these transfusions, no further blood was given. After June 1988 , the number of transfusions was reduced to one unit of blood. The leukocytepoor blood was prepared by removal of plasma and buffy coat by the local blood bank. Buffy coat was sent to the tissue typing laboratory; lymphocytes were isolated by Ficoll-Isopaque gradient centrifugation and stored in liquid nitrogen. A blood unit was transfused within $72 \mathrm{hr}$ of donation. Serum from the recipient was collected at weeks $0,2,3$, and 4 . Frozen cells from the transfusion donor were serotyped for HLA class I and II antigens and crossmatched with these sera. Positivity was defined as more than $20 \%$ cell death with the patient serum compared to the positive and negative control sera.

\section{Patients}

Nontransfused patients awaiting a first renal allograft $(\mathrm{n}=247 ; 225$ men and 22 women) have been transfused with at least one unit of leukocyte-poor erythrocytes. Blood donors were chosen randomly. To 93 patients, up to three blood transfusions were given with an interval of 1 month, as this was the center transfusion policy until 1988. After that date, the number of transfusions was reduced to one; therefore, 154 patients received one blood transfusion. None of the patients had preexistent HLA antibodies, and none of the female patients had been pregnant. Their mean age at time of transfusion was 46 years (SD: 14.1; median: 47; range: 15-72). Development of anti-donor antibodies was analyzed after the first transfusion.

Fifteen of the 154 patients received additional leukocyte-containing transfusions for clinical reasons during their time on the renal transplant waiting list. They were therefore excluded from further analysis concerning the influence of DR-matched transfusion on graft outcome. From 139 patients who each received one blood transfusion after June 1988, 103 patients ( 95 men and 8 women) subsequently received an unrelated transplant in the period from March 1989 through April 
1997. The mean age was 49.4 years (SD: 14.5 ; median: 49.3 ; range: 15-73). Additional blood transfusions were required in 49 patients. Only filtrated, leukocyte-depleted blood was used in those cases.

\section{Immunosuppression}

Patients were treated with either CsA $(n=84)$ or tacrolimus $(F K, n=19)$ together with low-dose prednisolone. Twenty-eight patients additionally received azathioprine $(n=22)$ or mycophenolate mofetil $(M M F)(n=6)$. Trough levels were determined in whole blood by high-performance liquid chromatography or monoclonal radioimmunoassay for CsA and by enzyme-linked immunosorbent assay (Imx, Abbott) for FK. The target level for CsA was $0.15-0.20 \mathrm{mg} / \mathrm{L}$ in the first 3 months after transplantation and $0.05-0.15$ after 3 months. The target level for FK was $15 \mathrm{ng} / \mathrm{mL}$ in the first months after transplantation, decreasing to $5-7 \mathrm{ng} / \mathrm{mL}$ after 1 year.

\section{Clinical outcome parameters}

Rejection is defined as any rejection treatment in the first 6 months after grafting. In general, rejections were proven by needle core biopsy. Rejection treatment consisted either of a 10-day course of rabbit anti-lymphocyte globulin (RIVM, Bilthoven, the Netherlands) or three doses of methylprednisolone $(0.5-1.0 \mathrm{~g} / \mathrm{dose})$. Graft failure was defined as loss of kidney function but also included death of recipient with functioning graft. No exclusions were made. All patients were followed until July I, 1997 or their date of death.

\section{Tissue typing and crossmatching}

Patients and blood donors were serologically typed for HLA antigens A, B, and DR by standard complement-dependent cytotoxicity assays (National Institutes of Health (NIH) for class I and two-color fluorescence (TCF) for class II). Screening was performed by the same techniques against a panel of 56 donor lymphocytes. A reaction with donor cells was considered positive if more than $20 \%$ of the lymphocytes were killed. Reactions were scored by automatic reading. The presence of autoantibodies was determined by crossmatching with autologous cells. Transfusion donor-specific crossmatches were performed in the standard NIH assay and the TCF technique for sera drawn at weeks $0,2,3$, and 4 . In some instances, the viability of the liquid-nitrogen-stored donor lymphocytes was insufficient to give reliable results; therefore, the number of crossmatches performed varied in the different groups. For crossmatches with the kidney donor, the dithiothreitol (DTT) crossmatch was also used, in which DTT was added to the serum to reduce IgM antibodies. A negative class I crossmatch $(\mathrm{NIH})$ with current as well as all relevant positive historical samples was mandatory for transplantation. All crossmatches as 
well as screening results were used in the interpretation of the crossmatch results, i.e., if a class I crossmatch turned negative after DTT treatment, the crossmatch was interpreted negative, provided no HLA-specific antibodies had been demonstrated by screening.

An antigen was considered matched if this antigen was present in donor as well as recipient. HLA antigens were considered unacceptable for a patient if antibodies against the specificity had been demonstrated either at the time of transplant or in the past. Transplants were performed under the auspices of Eurotransplant. Matching priority was HLA-DR, HLA-B, and HLA-A, respectively.

\section{Statistics}

Statistical analysis was performed using SPSS for Windows version 7.5. The proportional hazards regression analysis (Cox regression) was performed by both stepwise forward and backward selection techniques for all suspected risk factors except the HLA-DR match of transfusion donor, which was always included in each model. In case rejection was the outcome parameter, the suspected risk factors tested for included (besides HLA-DR match of transfusion donor) age of recipient, number of mismatches with the kidney donor for HLA-A, -B, and -DR $(0$ and $\geq 1$ ), source of kidney (spousal vs. cadaveric and heart-beating vs. non-heartbeating), type of primary immunosuppression (CsA vs. FK), institution of additional immunosuppressives (MMF vs. azathioprine vs. none), age of kidney donor, and the need for additional transfusion of filtrated blood after the protocollary transfusion (yes vs. no). Subsequently, a basic model was searched for, which included only direct risk odds ratio effects that were statistically significant. Thereafter, all interactions between pairs of basic model risk factors were introduced separately (and collectively) into the basic model and tested for statistical significance. Eventually, a final model is presented that only includes statistically significant risk factor effects and interactive effects. With graft survival as outcome parameter, rejection was also included as risk factor in the model. The same policy in model selection was followed for this outcome variable. Parametric (independent groups, Student's $t$-test) and nonparametric tests (Pearson chi-square test, Fisher's exact test) were performed when indicated. A $P$-value $<0.05$ was considered to be statistically significant.

\section{Results}

\section{Donor-specific antibody formation}

The development of donor-specific HLA class 1 antibodies after transfusion was shown in 16 patients $(6.5 \%)$ by a negative NIH crossmatch with the serum from 
week 0 and a positive crossmatch with the sera from weeks 2,3 and 4 . In the remaining 231 patients, the NIH crossmatches remained negative. The TCF crossmatch turned positive in 65 out of 242 patients $(26.9 \%)$ and remained negative in 177 patients $(73.1 \%)$.

Of 247 transfused patients, $140(56.7 \%)$ shared no DR antigen, 99 shared one DR antigen, and 8 shared two DR antigens. The one-DR- and two-DR-matched groups were combined to create the DR-matched group $(n=107)$ versus the nonmatched group $(n=140)$. There was no difference between matched and nonmatched group regarding the development of positive NIH crossmatches $(6.5 \%$ vs. $6.4 \%$, Table 2.1). TCF crossmatches turned positive in 41 patients in the nonmatched group $(30.1 \%)$ versus 24 patients in the DR-matched group $(22.6 \%)(P=0.24)$.

Table 2.1 Donor-directed antibody formation after transfusion

\begin{tabular}{lcccccc}
\hline & $\begin{array}{c}\text { NIH } \\
\text { (n) }\end{array}$ & $\begin{array}{c}\text { TCF } \\
\text { (n) }\end{array}$ & $\begin{array}{c}\text { NIH } \\
\text { negative }\end{array}$ & $\begin{array}{c}\text { TCF } \\
\text { negative }\end{array}$ & $\begin{array}{c}\text { NIH } \\
\text { positive }\end{array}$ & $\begin{array}{c}\text { TCF } \\
\text { positive }\end{array}$ \\
\hline DR sharing 0 & 140 & 136 & $93.6 \%$ & $69.9 \%$ & $6.4 \%$ & $30.1 \%$ \\
DR sharing 1/2 & 107 & 106 & $93.5 \%$ & $77.4 \%$ & $6.5 \%$ & $22.6 \%$ \\
Total & 247 & 242 & $93.5 \%$ & $73.1 \%$ & $6.5 \%$ & $29.6 \%$ \\
\hline
\end{tabular}

* NIH: $P=1.00$ (Fisher's Exact): TCF: $P=0.24$ (Fisher's Exact): positive $=$ more than $20 \%$ kill in the donor-specific crossmatch

\section{Autoantibodies}

Auto-crossmatches were performed for all sera used in the donor-specific crossmatches. None of the auto-crossmatches was found to be positive in the NIH technique. In 9 out of 242 patients, the auto-crossmatch by TCF could not be analyzed for technical reasons. Sixty out of 233 patients were demonstrated to have a positive auto-crossmatch. When the patients with a positive auto-crossmatch were excluded from the analysis, allo-antibodies were demonstrated in 18 out of 97 patients of the nonmatched group $(18.6 \%)$ and in 12 out of 76 patients in the matched group (15.8\%) $(P=0.69$, Table 2.2$)$.

Table 2.2 Formation of donor-directed antibodies after one transfusion corrected for the presence of auto-antibodies"

\begin{tabular}{lcccccc}
\hline & \multicolumn{2}{c}{ Total } & \multicolumn{2}{c}{ TCF negative } & \multicolumn{2}{c}{ TCF positive } \\
& $\mathbf{n}$ & $\%$ & n & $\%$ & n & $\%$ \\
\hline DR sharing 0 & 97 & 56.1 & 79 & 81.4 & 18 & 18.6 \\
DR sharing 1/2 & 76 & 43.9 & 64 & 84.2 & 12 & 15.8 \\
Total & 173 & 100 & 143 & 82.7 & 30 & 17.3 \\
\hline
\end{tabular}

* $P=0.69$ (Fisher's Exact) 


\section{Antibody formation according to sharing for class I and class II antigens}

In 244 patients, the correlation between sharing for HLA class I and II antigens with the transfusion donor was analyzed. Sharing for HLA-A was not correlated with sharing for either $\mathrm{B}$ or $\mathrm{DR}(P=0.50$ and $P=0.77$, respectively). Sharing for HLA-B was shown to be correlated with sharing for HLA-DR, as might be expected. Seven patients shared two B antigens $(2.9 \%), 73$ shared one B antigen $(29.9 \%)$, and 164 shared none $(67.2 \%)$. The percentage of nonmatched HLA-DR patients was $43.4 \%$ in the B-matched group vs. $62.8 \%$ in the B-nonmatched group ( $P=0.006$ by Fisher's exact test). Sharing for A plus B antigens was also significantly correlated with match for DR ( $P=0.04$ by Spearman correlation).

The correlation between sharing for $\mathrm{A}, \mathrm{B}, \mathrm{A}+\mathrm{B}$, and $\mathrm{B}+\mathrm{DR}$ and the formation of class I antibodies $(\mathrm{NIH})$ are given in Table 2.3, part A. The results for class II antibodies (TCF) are given in Table 2.3, part B. Formation of antibodies was in no combination significantly related to sharing of HLA antigens.

Table 2.3 Donor-directed antibody formation by NIH (part A) or by TCF (part B) after one transfusion according to HLA sharing

\begin{tabular}{|c|c|c|c|c|c|c|c|c|c|c|}
\hline & \multicolumn{2}{|c|}{ HLA-A } & \multicolumn{2}{|c|}{ HLA-B } & \multicolumn{3}{|c|}{ HLA-AB } & \multicolumn{3}{|c|}{ HLA-BDR } \\
\hline & 0 & $1 / 2$ & 0 & $1 / 2$ & 01 & 1A & IB & 01 & IB & IDR \\
\hline \multicolumn{11}{|l|}{$\mathbf{A}^{*}$} \\
\hline NIH negative & $94 \%$ & $93 \%$ & $93 \%$ & $95 \%$ & $92 \%$ & $95 \%$ & $93 \%$ & $92 \%$ & $95 \%$ & $93 \%$ \\
\hline NIH positive & $7 \pi$ & $7 \%$ & $7 \%$ & $5 \%$ & $8 \%$ & $5 \%$ & $7 \%$ & $8 \%$ & $5 \%$ & 78 \\
\hline Total & 123 & 121 & 164 & 80 & 86 & 115 & 43 & 103 & 96 & 45 \\
\hline \multicolumn{11}{|l|}{$\mathrm{B}^{\mathrm{b}}$} \\
\hline TCF negative & $74 \%$ & $72 \%$ & $74 \%$ & $70 \%$ & $74 \%$ & $74 \%$ & $67 \%$ & $69 \%$ & $79 \%$ & $69 \%$ \\
\hline TCF positive & $26 \%$ & $28 \%$ & $26 \%$ & $30 \%$ & $26 \%$ & $26 \%$ & $33 \%$ & $31 \%$ & $21 \%$ & $31 \%$ \\
\hline Total & 122 & 117 & 160 & 79 & 85 & 112 & 42 & 100 & 94 & 45 \\
\hline
\end{tabular}

- HLA-A: $P=1.00$ (Fisher's Exact), HLA-B: $P=0.59$ (Fisher's Exact): HLA-AB: $P=0.70$ (Pearson chi-square); HLA-BDR: $P=0.77$ (Pearson chi-square)

b HLAA: $P=0.77$ (Fisher's Exact); HLA-B: $P=0.44$ (Fisher's Exict); HLA-AB: $P=0.62$ (Pearson chi-square): HLA-BDR: $P=0.25$ (Pearson chi-square)

\section{Relationship between DR sharing of transfusion donor/recipient and clinical outcome after transplantation}

Out of 139 patients who received one leukocyte-poor blood transfusion, 103 patients have received transplants. Fifty-nine patients have received a nonmatched DR transfusion $(57.3 \%)$ and 44 patients $(42.7 \%)$ a matched one. There was no significant difference between these groups for any parameter shown in Table 2.4. 
Table 2.4 Basic characteristics of 103 renal allograft recipients according to number of HLA-DR matches with transfusion donor.

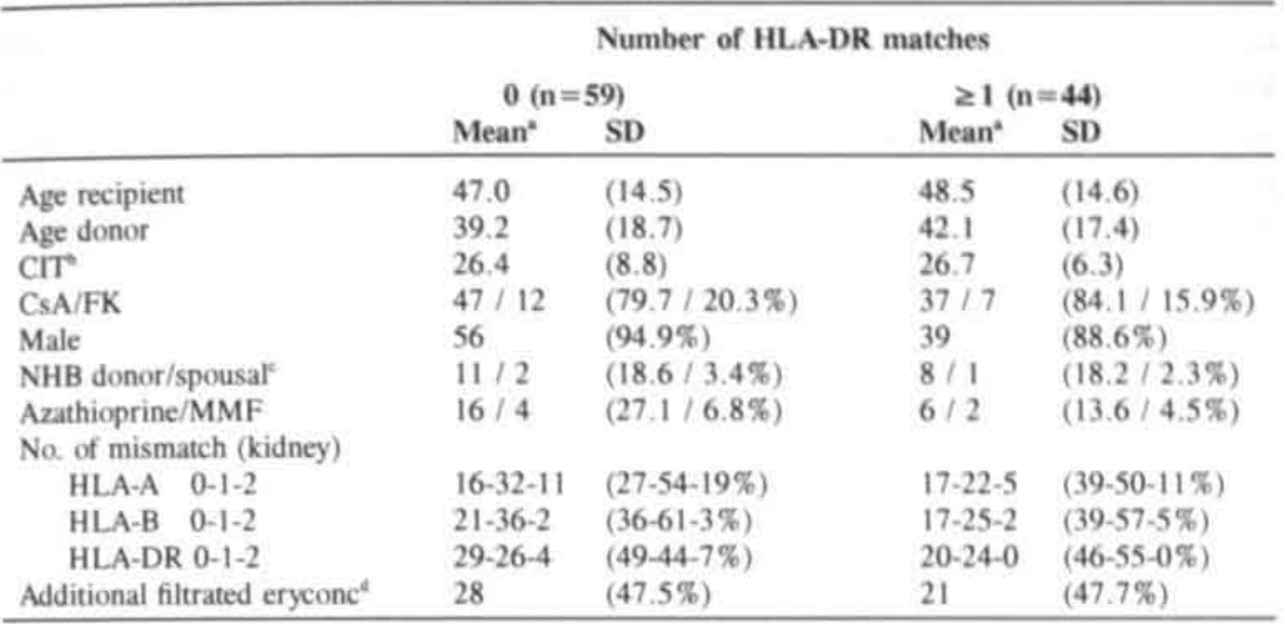

- Data are given as numbers or mean $\pm \mathrm{SD}$

' CIT, cold ischemia time

"NHB, non-heartbeating

"Eryconc, erythrocytes concentration.

\section{Graft survival}

Figure 2.1 depicts the graft survival rate (Cox regression model plot) for the 103 patients who received transplants according to DR match with the transfusion donor. In the Cox regression analysis, DR matching did not contribute significantly towards graft survival $(P=0.97$; odds ratio: $1.02 ; 95 \%$ confidence interval $(\mathrm{CI})$ : $0.45-2.32$ ). There also was no significant contribution towards graft survival for any of the other riskfactors (age of recipient, number of mismatches with the kidney donor for HLA-A, -B, and -DR, source of kidney, type of primary immunosuppression, institution of additional immunosuppressives, age of kidney donor, the need for transfusion of filtrated blood, and the occurrence of rejection).

\section{Rejection-free survival}

In the Cox regression analysis, DR match with the transfusion donor did not contribute significantly towards rejection ( $P=0.99$; partial odds ratio: $1.0 ; 95 \% \mathrm{CI}$ : $0.5-2.0$ ). Risk factors that did contribute significantly towards rejection were type of primary immunosuppression and mismatch for HLA-DR with kidney donor. The partial Odds-ratio for rejection was 4.4 (95\% CI: 1.1-18.6) in patients using CsA versus FK, and 2.9 (95\% CI: 1.3-6.3) in patients transplanted with a kidney with a DR mismatch versus no DR mismatch. The contribution measured as a change in 
-2LL of type of primary immunosuppression, given a model including only DR match with the transfusion donor and mismatch for HLA-DR with kidney donor is 6.35 (with $1 \mathrm{df}, P=0.012$ ), and the contribution of mismatch for HLA-DR with kidney donor given a model including only DR match with the transfusion donor and type of primary immunosuppression is 7.87 (with $1 \mathrm{df}, P=0.0005$ ). So, next to DR match with the transfusion donor, both risk factors are necessary to the basic model. Basic model -2LL change amounts to 11.7 ( $3 \mathrm{df}, P=0.008)$, and this model is at the same time the final model because interactions between the three risk factors turned out to be nonsignificant. Figure 2.2 depicts the rejection-free survival (Cox regression model plot) for the 103 transplanted patients according to DR match with the transfusion donor.

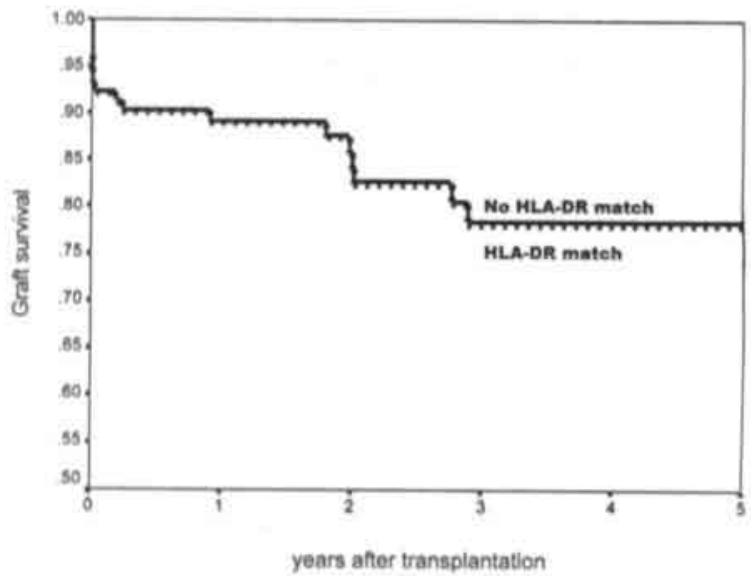

Figure 2.1 Predicted graft survival based on HLA-DR match with transfusion donor. Cox regression model plot by risk factor HLA-DR match of transfusion donor.

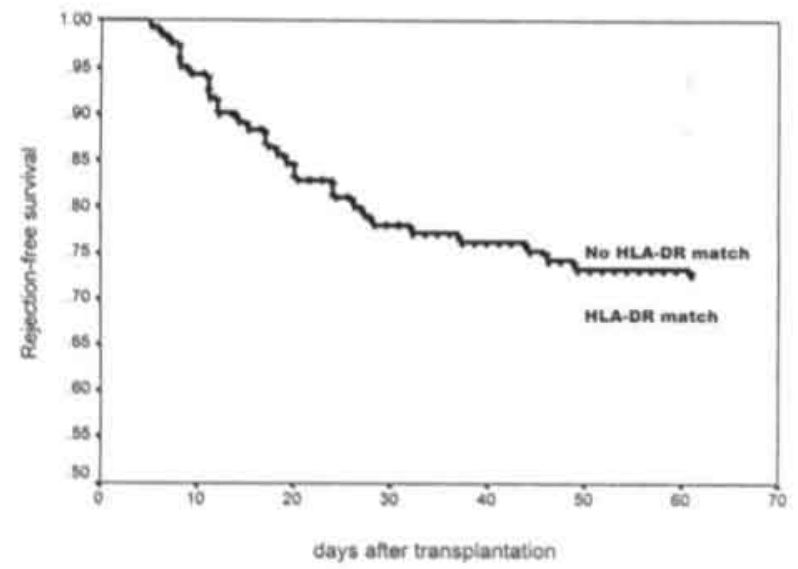

Figure 2.2 Predicted rejection-free survival based on HLA-DR match with transfusion donor. Cox regression model plot by HLA-DR match of transfusion donor with risk factors HLA-DR mismatch between kidncy donor and recipient $(0 \mathrm{vs} \geq 1)$ and type of primary immunosuppression (CsA vs. FK). 


\section{Discussion}

Decreased formation of antibodies and improved renal graft survival as a result of matching for HLA-DR between transfusion donor and recipient have been reported $^{\text {t9.9}}$. In these studies, the presence of cytotoxic antibodies was defined by screening of the recipient sera with a panel of lymphocytes. In the present study, a different approach was used and development of antibodies was measured by performing donor-specific crossmatches. The antibodies were studied by performing both the NIH (T cell) and TCF (B and T cells) crossmatches with transfusion donor lymphocytes. All sera were also investigated with autologous lymphocytes to determine the presence of autoantibodies. All 247 patients included in this study were so-called "naive patients", i.e., had not been in contact with nonself-HLA antigens.

This study shows no difference in occurrence of cytotoxic antibodies between patients transfused with a HLA-DR-matched or nonmatched transfusion. These results are in accordance with several studies that showed an increase in the frequency of cytotoxic $T$ cell precursors several weeks after transfusion, irrespective of the HLA-DR match of the transfusion ${ }^{12-14}$.

For all studies on the transfusion of HLA-DR-matched blood, it should be kept in mind that the composition of a unit of blood is not standardized, i.e., there are no firm data about the necessary number of leukocytes in a unit, nor is the optimal time period between donation and transfusion known to accomplish the beneficial effect.

The transfusion studies described so far differ in a number of respects. The type and the amount of blood given are different. Leukocyte-depleted blood was most often used ${ }^{7.8}$; occasionally, whole blood was transfused ${ }^{7.9}$. There were differences in the number of transfusions administered. Lagaaij transfused the heart and kidney patients in the retrospective part of her study with 1 unit of blood but used 3 units in the prospective kidney study ${ }^{7}$. Middleton used 2 units of blood ${ }^{8}$, while Bayle gave 1 transfusion to his matched group of patients and 3 to the random group. It is obvious that transfusion of 2 or 3 bloodunits will influence the prevalence of HLA antibodies ${ }^{10.11}$. In the present study, leukocyte-depleted blood was administered within 72 hours after donation, to secure donation of viable lymphocytes ${ }^{5.6}$. We analyzed patients after transfusion of 1 unit of blood.

It is not known whether the difference between DR-matched and DR-nonmatched blood is a result of the fact that patients received a matched transfusion (irrespective of the number of mismatched transfusions) or to the fact that patients received a nonmatched transfusion (irrespective of the number of matched transfusions). This might be important because both Bayle and Middleton 
transfused random units of blood to patients in the control group. In that way, theoretically, as stated by Middleton, over $60 \%$ of the patients received a transfusion with DR-matched blood ${ }^{8}$, while nearly all control patients received a DR-nonmatched transfusion. These differences make the results difficult to compare.

Middleton demonstrated antibodies in $15-21 \%$ of the patients by the NIH technique after two transfusions. The difference between patients receiving matched and nonmatched blood was not statistically significant. Bayle reported antibodies by NIH in $17 \%$ of the 48 naive patients that received three random transfusions. Lagaaij detected NIH antibodies in $12.5 \%$ of the 16 patients who received three DR-shared transfusions in her study on kidney transplant recipients, but reported a percentage as high as $75 \%$ in 16 patients receiving three nonmatched transfusions. Bayle found no antibodies in 36 naive patients who recieved one unit of haplo- or semi-identical blood. The higher frequency of antibodies in his control group could be the effect of the number of transfusions rather than the matching. The additional effect of the haplo-(or semi-)identical transfusion is unclear. The number of naive patients in the studies mentioned are small (32-84 patients). In the present study, comprising 247 patients, the percentage of antibodies detected by NIH donor crossmatches was $6.5 \%$, which is lower than in the above mentioned studies. The difference might be explained by differences in detection (donor-specific vs. PRA), definition of positivity, or type, quantity, and quality of the transfused blood.

Besides the NIH technique, Middleton also used CLL cells for screening the recipient sera and thus detected HLA class II antibodies. Antibodies reactive with CLL cells were detected in $16-21 \%$ of the patients. CLL cells are reported to be insensitive to auto-antibodies. In our study, the occurrence of antibodies detected by the TCF technique was $26.9 \%$. After excluding the patients with autoantibodies, we obtained a comparable percentage of $17.3 \%$ B-cell-positive patients. In both studies, the percentage of patients who developed antibodies against B cells was not significantly different between the DR-matched and the DR-nonmatched transfusion group. This is in contrast to our earlier findings in a smaller patient series, where patients receiving DR-nonmatched transfusions demonstrated a higher percentage of B-cell antibodies ${ }^{15}$. In the current extended study, we could not confirm the earlier findings.

Finding a lower frequency of antibodies might be the result of missing transient antibodies. Dependent on the date the sera were drawn and the number of sera tested, antibodies can be missed. Middleton and Lagaaij tested one serum drawn 2 weeks after transfusion and Bayle tested four sera drawn in the first month after the transfusion. We used three sera drawn on week 2,3 , and 4 after transfusion and tested them directly against the immunizing donor. We therefore think that it is 
unlikely that transient antibodies have been overlooked. The higher percentages in other studies might be a result of aspecific panel reactivity or the presence of autoantibodies.

A positive influence of DR matching on graft survival was only reported by Lagaaij ${ }^{7}$. She mentioned a better graft survival in 32 patients who had received a DR-matched transfusion compared to 30 patients who received a DR-nonmatched transfusion. Remarkably, the frequency of antibodies after transfusion was not mentioned by Lagaaij, nor was the rate of rejection and the cause of failure (immunological/non-immunological). Immunosuppression consisted of azathioprine and prednisolone for both patient groups. None of the other studies, including this one, was able to show a difference in graft survival in patients immunosuppressed with CsA or FK. In the 1970s and 1980s, kidneys were matched primarily on the B locus and not DR; therefore, patients receiving a matched transfusion were probably patients with a frequent HLA-DR antigen. It is also probable that they more often received a DR-matched kidney. This could explain the better results of the DR-matched group on graft outcome. The fact that there was no significant difference in mean number of mismatches for HLA-DR does not mean that the distribution of DR mismatches was equal. The beneficial influence of HLA-DRmatched kidneys on graft survival has been proven, especially for the zero DR mismatch group. The analysis was not multivariate, so it is unknown whether other factors may explain the worse outcome in the non-matched group.

An effect of DR sharing on rejection was noticed by both Middleton and Bayle ${ }^{8.9}$. In their respective studies, they noticed a decrease in the frequency of rejection by DR matching. However, Middleton as well as Bayle used a historical control group for which the match grade of the transfusion donors was unknown. Over $60 \%$ of the control patients in both studies are expected to have received at least one DRmatched transfusion; therefore, the comparison is not really DR-matched versus DR-nonmatched transfusions. Furthermore, actuarial survival (Kaplan-Meier) analysis was used, ignoring important co-variates for rejection such as, for example, matching with the kidney donor. As stated above for graft survival, the fact that the mean number of mismatches with the kidney donor was not significantly different between both groups does not necessarily mean that the distribution of the number of mismatches is equal. In the present study, the data were analyzed by Cox regression, which implicates correction for confounding factors. As matching priority was given to the HLA-DR locus, patients received DR well-matched kidneys irrespective of the DR match of the transfused blood.

In conclusion, in this study for the first time prospective donor-specific crossmatches were used for the detection of the HLA antibody frequency after one 
transfusion in naive patients awaiting renal transplantation. Retrospectively the influence of DR matching on the formation of HLA antibodies was analyzed. The influence of DR-matched transfusions on antibody formation and graft outcome in renal transplant candidates was analyzed on the largest number of patients reported so far. We did not find a significant difference in the occurrence of HLA antibodies between patients receiving DR-matched and DR-nonmatched blood, which is in accordance with Middleton's results. This held for antibodies against $\mathrm{T}$ cells (detected by $\mathrm{NIH}$ ) and for antibodies reactive with B cells (detected by TCF). The anti T-cell antibodies detected were all allo-antibodies. About $25 \%$ of the sera showed autoreactivity with B-cells. These figures were the same in both matching groups. Also, in graft outcome, no difference was shown in rejection rate or graft survival up to 5 years after transplantation between both matching groups. Factors influencing rejection were well known factors as type of immunosuppression and HLA-DR matching with kidney donor. Therefore, we were not able to confirm the data reported by Lagaaij, Bayle, and Middleton.

At this moment, we cannot recommend transfusion of DR-matched blood for pretransplant transfusion in view of the additional costs and efforts it requires. However, a prospective randomized study, comparing DR-matched and DR-nonmatched blood transfusions, will be the only way to obtain a clear-cut answer on the question whether DR-matched transfusions have a beneficial effect on transplantation. According to our data, such a study should include several hundred patients, to obtain statistically significant differences in occurrence of antibodies, rejection rate, and/or graft survival. 


\section{References}

1. Opelz G, Sengar DPS, Mickey MR, Terasaki PI. Effect of blood transfusions on subsequent kidney transplants. Transplant Proc 1973; 5: 253-9.

2. Opelz G, Vanrenterghem Y, Kirste G, Gray DW, Horsburgh T, Lachance JG, Largiader F, Lange H, Vujaklija-Stipanovic K, Alvarez-Grande J, Schott W, Hoyer J. Schnuelle P, Descoeudres C, Ruder H, Wuiciak T, Schwarz V. Prospective evaluation of pretransplant blood transfusions in cadaver kidney recipients. Transplantation 1997;63: 964-7.

3. van Hooff JP, Kalff MW, Poelgeest AE, Persijn GG, van Rood J. Blood transfusions and kidney transplantation. Transplantation 1976: 22: 306-7.

4. Persijn GG, Cohen B, Lansbergen Q, van Rood JJ. Retrospective and prospective studies on the effect of blood transfusions in renal transplantation in the Netherlands. Transplantation 1979; 28: 396-401.

5. Nube MG, Persijn GG. Kalff MW. Kidney transplantation: presensitization and transplant survival; clinical aspects. Neth J Med 1977; 20: 283-8.

6. Fuller TC, Delmonico FL, Cosimi AB, Huggins CE, King M, Russell PS. Effects of various types of RBC transfusions on HLA alloimmunization and renal allograft survival. Transplant Proc 1977; 9: 117-9.

7. Lagaaij EL, Hennemann IPH, Ruigrok M, de Haan MW, Persijn GG, Termijtelen A, Hendricks GF, Weimar W, Claas FH, van Rood JJ. Effect of one-HLA-DRantigen-matched and completely HLA-DR-mismatched blood transfusions on survival of heart and kidney allografts. NEJM 1989; 321: 701-5.

8. Middleton D, Martin J, Douglas J, McClelland M. Transfusion of one HLA-DR antigen-matched blood to potential recipients of a renal allograft. Transplantation 1994; $58: 845-8$.

9. Bayle F, Masson D, Zaoui P, Vialtel P, Janbon B, Bensa J-C, Cordonnier DJ.. Beneficial effects of one HLA haplo- or semi-identical transfusion versus three untyped blood units on alloimmunization and acute rejection episodes in first renal allograft recipients. Transplantation 1995; 59: 719-23.

10. Opelz G, Mickey MR, Terasaki PI. Blood transfusions and unresponsiveness to HLA. Transplantation 1973; 16: 649-54.

11. van den Berg-Loonen PM, Leunissen KML, Kootstra G, van Hooff JP. B-cell antibodies after planned transfusions. Transplant Proc 1987; 19: 743.

12. Baudouin V, de Vitry N, Hiesse C, Lang P, Bloch J, Legouvello S, Sterkers G. Cytotoxic T lymphocyte changes after HLA-DR match and HLA-DR mismatch blood transfusions. Transplantation 1997; 63: 1155-60.

13. Young NT, Roelen DL, Iggo N, Gray DW, Roake JA, Graham V, Wood KJ, Dallman MJ, Welsh KI, Morris PJ. Effect of one-HLA-haplotype-matched and HLA-mismatched blood transfusions on recipient $T$ lymphocyte allorepertoires. Transplantation 1997; 63: 1160-5. 
14. Mast BJ, Viëtor HE, van der Meer-Prins EMW, van Bree SP, Brand A, van den Elsen PJ, Claas FH. Modulation of the T cell compartment by blood transfusion: effect on cytotoxic and helper $\mathrm{T}$ lymphocyte precursor frequencies and $\mathrm{T}$ cell receptor VB usage. Transplantation 1997; 63: 1145-54.

15. van Hooff JP, van den Berg-Loonen PM. The influence of DR-match of blood donor and recipient on the formation of T- and B-cell antibodies and on renal allograft outcome. Transpl Int 1992; 5(S1): 599-600. 


\section{Chapter 3}

\section{HLA class I and II antibodies by ELISA and CDC before and after transplantation}

MHL Christiaans, F Nieman, JP van Hooff, EM van den Berg-Loonen

Transplantation (in press) 


\section{Abstract}

\section{Background}

Anti-class I IgG can be detected by CDC and ELISA. We compared ELISA and for CDC both class I and II antibodies on method agreement and relation to rejection-free and graft survival.

\section{Methods}

Peak, current, and post-transplant sera $(n=429)$ of 143 renal allograft patients were tested by NIH, TCF, and ELISA. Method agreement was assessed by intraclass correlation coefficient (ICC), and rejection and graft survival were analyzed by uni- and multivariate techniques. The screening results for each serum were compared, as was change in result of current to post-transplant serum.

\section{Results}

ICC of ELISA and NIH was insufficient, it was lower for TCF than for NIH. Graft survival was not related to any assay.

Rejection-free survival was related to ELISA and NIH in current and post-transplant serum. For NIH, the change in \%PRA correlated better with rejection than for ELISA. The combined antibody status of current and post-transplant serum was a risk factor for rejection for all assays, and for TCF also in multivariate analysis. The rejection rate was higher when the post-transplant serum was ELISA-negative/CDC-positive than ELISApositive/CDC-negative. For ELISA, class I specificities and not \%PRA in peak and current serum were related to rejection, even when the antibodies were not donordirected. For NIH, \%PRA and not specificity was related to rejection. Class II antibodies were never related to rejection.

\section{Conclusions}

ELISA and NIH are complementary screening techniques in this patient population. They are of equal predictive value for rejection. The optimal strategy in combining these techniques has yet to be determined. 


\section{Introduction}

Preformed anti-HLA class I antibodies directed against mismatched donor-antigens are detrimental to the function of the renal graft when they belong to the $\operatorname{lgG}$ immunoglobulin class. The presence of these antibodies is correlated with rejection and graft failure ${ }^{1.2}$. Although there is no consensus on the influence of HLA-class II antibodies, association with graft failure has been reported ${ }^{3.4}$. The influence of HLA-specific antibodies of the IgM class is still not clear, but reports associated with graft failure have been published ${ }^{2.5}$. Autoantibodies, which are usually of the lgM class, do not affect transplant outcome ${ }^{4,6}$.

A proper definition of antibody specificity is essential for the interpretation of a positive crossmatch. Therefore, the sera of patients on the waiting list for renal transplantation are routinely screened for the presence and specificity of HLAdirected antibodies before transplantation. After transplantation, patient sera are investigated to monitor the graft immunologically. Traditionally, the screening technique has been the complement-dependent cytotoxicity (CDC) assay. With this technique, complement-fixing T-cell-reactive antibodies of the IgG1, IgG2, and IgM classes are detected including non-HLA and autoantibodies. The assay requires the availability of a large panel of viable phenotyped cells. To increase the sensitivity of the technique, several modifications have been made, such as extension of the number of wash steps, an increase in the incubation times and the addition of DTT (dithiotreitol). Positive reactions due to $\mathrm{IgM}$ antibodies and autoantibodies are avoided in the presence of DTT. The addition of anti-human globulin (AHG) increases the sensitivity of the test. By using B lymphocytes instead of T lymphocytes as target cells, HLA class II antibodies can also be detected, in addition to IgM and complement-fixing non-HLA antibodies. The results of the different assays are usually expressed as the percentage of cells reactive with the tested serum, the \%PRA (panel reactive antibodies), and as HLAspecificity. The \%PRA depends on the composition and the size of the cell panel used.

An alternative assay based on the ELISA technique Prastat, has been introduced. Soluble HLA antigens are coated onto ELISA plates and incubated with serum. HLA-specific immunoglobulin in the test serum binds to the HLA antigens and bound IgG is made visible by an enzymatic coloring technique. Prastat detects HLA-specific antibodies of the $\operatorname{lgG}$ class including non-complement-fixing antibodies, but not IgM. Originally, the assay was designed for HLA class I-specific antibodies; however, it was recently observed that HLA class II antibodies are also detected. 
mandatory for the transplantation to be performed; this held for current as well as for historical sera. In the NIH crossmatch, the presence of donor-directed antibodies in the recipient serum was defined as cell death $>20 \%$ above background. Screening results were used in the interpretation of the crossmatch.

\section{Antibody screening}

\section{Sera}

In this study, the following sera were used in CDC and ELISA for each patient: (1) current serum, (2) peak (historical) serum, and (3) post-transplant serum. The current serum was drawn at the time of transplantation. The peak serum was selected as the historical serum with the highest panel reactivity by $\mathrm{NIH}$; for antibody-negative patients, the serum drawn 4 weeks after the pretransplant blood transfusion was considered the peak serum. For retransplant patients without HLA antibodies, the peak serum used had a bleeding date after failure of the previous graft, preferably after transplantectomy. Post-transplant sera were obtained within 6 months after transplantation, in the case of rejection after rejection, and in the case of transplantectomy between 2-4 weeks later. Sera drawn within 10 days after the last administration of OKT3 were excluded to avoid falsepositive results ${ }^{17}$. All sera were also investigated for the presence of autoantibodies by performing crossmatches with liquid-nitrogen-stored peripheral blood lymphocytes of the recipient.

\section{Screening by complement-dependent-cytotoxicity}

Screening for the presence of anti-HLA class I antibodies was performed with and without the addition of DTT using a selected panel of 64 lymphocytes. Most HLA antigens were represented three times. The presence of class II antibodies was established at the same time and from the same wells, but with a different positive control for the calculations. Briefly, $1 \mu \mathrm{L}$ of test serum was added to the cell suspension in Terasaki plates. Control sera on each plate were $3 \mathrm{HLA}-\mathrm{ABC}$ and 3 HLA-DR-multispecific antisera, 3 negative sera, and 1 specific positive HLA class I or II antiserum. Cells and serum were incubated for 45 minutes at $20^{\circ} \mathrm{C}$ in the dark. Rabbit complement $(2.5 \mu \mathrm{L}$, Bioscope, Wageningen $)$ was added and incubated for 105 minutes at $20^{\circ} \mathrm{C}$ in the dark. After the addition of propidiumiodide ink $(2.5 \mu \mathrm{L})$, the plates were read. Reading was performed via a Leitz Patimed automated microfluorometer microscope, and positivity of a well was defined as $>40 \%$ cell death of the patient serum compared to three positive control sera $(100 \%)$. The $40 \%$ value was established by extended comparison of the microfluorometer microscope results with the previously used classical NIH technique in which the results were obtained by eye reading (positivity $\geq 10 \%$ ). The percentage of cells positive with the test serum (PRA) was reported separately for $T$ cells and $B$ cells. The specificity of the antibodies was analyzed by 
comparing the positive reaction pattern with the HLA profile of the cells in the cell panel. The results of the assays were expressed as \%PRA and as positive or negative. In NIH, a result was interpreted as negative if $<10 \%$ of the panel cells had a positive reaction; for TCF, a result $<25 \%$ was considered negative.

\section{Screening by ELISA}

The ELISA technique used was the commercially available Prastat (Sangstat Medical Corporation, Menlo Park, CA, USA). Soluble HLA (sHLA) molecules are recovered from the supernatant of 44 Epstein-Barr virus-transformed, HLAphenotyped cells. A 96-well microtiter plate is precoated with a pan-HLA monoclonal antibody (TP25) directed towards the alpha-3 domain of the HLA class I molecule. Originally, Prastat was claimed to contain only HLA class I molecules; however, it was shown to also contain HLA class II molecules. It is not clear in which way these HLA class II molecules are captured on the ELISA plate. Presented on the plates are 44 specimens of sHLA molecules, together with two positive controls, one negative control and one no-antigen control.

The test was performed according to the manufacturer's instructions. In short, 100 $\mu \mathrm{L}$ of the serum sample (dilution 1:101) was dispersed into each well and incubated for 2 hours at $21^{\circ} \mathrm{C}$. Positive and negative controls were used in each run. After three washes with an automatic plate-washer, $100 \mu \mathrm{L}$ of diluted peroxidaseconjugated goat-antihuman-lgG was added and the plates were again incubated for 1 hour. The unbound conjugate was removed by three wash steps, and $100 \mu \mathrm{L}$ O-phenylenediamine dihydrochloride was added as chromogenic substrate and then incubated for 15 minutes at $21^{\circ} \mathrm{C}$ in the dark. The reaction was stopped by adding $100 \mu \mathrm{L} 1 \mathrm{~N} \mathrm{HCl}$ and the plates were read on a Dynatech MR 5000-plate reader (Chantilly, VA). The run was considered valid if the results of the negative and positive controls fell within the limits specified by the manufacturer. A delta value was calculated for each well as the optical density (OD) of the test well minus the $\mathrm{OD}$ of the no-antigen well. A well was considered positive if the delta value of the well was $\geq 0.35$ times the mean delta value of the positive control wells.

Data were analyzed with SOFTSTAT II software (Sangstat, Medical Corporation, Menlo Park, CA, USA). The percentage of wells with a delta value in the positive range was calculated, as were the HLA class I and II antibody specificities. The result was considered negative for ELISA-PRA when $<10 \%$ of the wells showed a positive reaction.

\section{Clinical outcome parameters}

Rejection was defined as any rejection treatment in the first 6 months after grafting. There was clinical suspicion of rejection in the case of an unexplained rise or insufficient decrease in serum creatinine, with or without other signs such as fever, tenderness of the graft, decreased renal perfusion on nuclear scan, or 
hypertension. Unless there was a contraindication for biopsy (e.g., bleeding disorder, uncontrolled severe hypertension, neonatal kidney), a needle core biopsy was performed for confirmation. In the case of delayed graft function (need for dialysis in the first week after transplantation), a protocol biopsy was performed at week 1 to rule out rejection as the cause of non-function of the graft. Rejection treatment consisted of a 10-day course of rabbit anti-lymphocyte globulin (RIVM, Bilthoven, the Netherlands) or OKT3-Orthoclone (Jansen Cilag, Tilburg, the Netherlands). For patients who had received rabbit antilymphocyte globulin in the past or who had a seriously compromised cardiovascular system, rejection was treated with three doses of methylprednisolone $(0.5-1.0 \mathrm{~g} /$ dose $)$. Graft failure was defined as loss of kidney function, but also included death of the recipient with a functioning graft. No exclusions were made. All patients were followed up until January 1, 1996 or their date of death.

\section{Statistics}

Statistical analysis was performed using SPSS for Windows, version 7.5. Associations between antibody positivity or percentage of PRA measured by ELISA, NIH, and TCF were estimated using Spearman's rho. The intraclass correlation coefficient (ICC) was established with the results expressed as percentage PRA, using variance components generated from repeated measurements ANOVA. ICC is interpreted as a measure of agreement between methods and varies from 0 (no agreement at all) to 1 (perfect agreement) ${ }^{18}$. The lowest acceptable method agreement was defined at 0.85 .

For analysis of rejection-free and graft survival, the proportional hazards regression analysis (Cox regression) was performed, both by stepwise forward selection and by backward elimination techniques for all suspected risk factors except the result of the assay. In case rejection was the outcome parameter, the suspected risk factors tested for included: age and gender of recipient, age and gender of donor, transplant number, cause of death of donor (trauma vs. CVA vs. intracranial bleeding vs. other), non-heart-beating donor (yes vs. no), first and second warm ischemia time, cold ischemia time, number of HLA-A, -B, and -DR mismatches, cause of renal failure recipient, additional use of azathioprine (yes vs. no), type of dialysis pretransplant (hemodialysis vs. peritoneal dialysis vs. none), occurrence of delayed graft function.

A basic model was tested for that included only direct odds ratio effects that were statistically significant. Subsequently, all possible interaction terms representing combinations of basic effects were introduced separately and hierarchically into this model. If interactions were statistically significant, they were included in the definitive model. The result of the assay was defined as a single risk factor and subsequently introduced as a risk factor in addition to the basic model. In this way, the additional predictive value of the assay could be tested for under conditions of 
relevant risk factors.

With graft survival as outcome parameter, 'rejection' was included as a potential risk factor in the model. For this outcome variable, the same policy for model selection was followed as for rejection-free survival. Non-parametric tests (Pearson chi-square test, Fisher's exact test, Kruskal-Wallis test) were performed when indicated. A $P$-value $<0.05$ was considered to be statistically significant.

\section{Results}

\section{Method agreement between ELISA and CDC}

The results of 429 sera from 143 renal transplant patients screened with ELISA and CDC techniques are shown in Table 3.1. Sera were drawn before and after transplantation and represented the peak, current, and post-transplant serum of each patient. The results of the assays were expressed as the number of antibodypositive patients and as percentage of cells reactive with the serum (PRA). Both \%PRA and number of antibody-positive patients were similar for ELISA and NIH in current sera, for TCF they were higher. In Table 3.2, rank correlations and method agreement between ELISA and NIH/TCF are shown. The results showed that all correlations between ELISA and CDC were statistically significant $(P<0.001)$. ELISA had a higher rank correlation with NIH than with TCF as shown by the rho values. Method agreement, expressed as intraclass correlation coefficient (ICC), indicated insufficient agreement for one assay to be replaced by the other. This is illustrated in Figure 3.1, which shows the PRA dotplot for NIH versus ELISA of current sera. Since lack of agreement could be due to the presence of autoantibodies not detected in the ELISA assay, auto-crossmatches were performed with recipient lymphocytes in both NIH and TCF. Results were reanalyzed excluding autoantibody-positive sera; consequently, the total number of patients varied depending on the number of sera excluded (Table 3.2). The analysis showed that exclusion of autoantibody-positive sera did not improve either the correlation or the method agreement between ELISA and NIH or TCF.

Table 3.1 Antibody screening by ELISA, NIH, and TCF of peak, current, and postransplant sera of 143 renal transplant ptients.

\begin{tabular}{lllllll}
\hline & \multicolumn{2}{c}{ Peak serum } & \multicolumn{2}{c}{ Current serum } & \multicolumn{2}{c}{ Post-transplant serum } \\
& Pos pat & \%PRA (SD) & Pos pat & \%PRA (SD) & Pos pat & \%PRA (SD) \\
\hline ELISA & $56(39.2 \%)$ & $21 \%(30.4)$ & $46(32.2 \%)$ & $17 \%(27.3)$ & $52(36.4 \%)$ & $19 \%(29.6)$ \\
NIH & $60(42.9 \%)$ & $24 \%(34.7)$ & $44(30.8 \%)$ & $17 \%(28.9)$ & $60(42.0 \%)$ & $25 \%(35.3)$ \\
TCF & $80(55.9 \%)$ & $44 \%(41.8)$ & $61(42.7 \%)$ & $31 \%(36.0)$ & $84(58.7 \%)$ & $49 \%(41.5)$ \\
\hline
\end{tabular}

Results for each assay are expressed as number of positive patients and mean FPRA with standard deviation per serum. Antibody positivity is defined as $>10 \%$ PRA in ELISA and NIH, and $>25 \%$ in TCF. 
Table 3.2 Correlation between ELISA and NIH/TCF with peak, current, and post-transplant sera in 143 renal transplant recipients before (2a) and after (2b) exclusion of autoantibodies.

\begin{tabular}{lcccccccc}
\hline 2a & \multicolumn{3}{c}{ NIH (\%PRA) } & \multicolumn{3}{c}{ TCF (\%PRA) } \\
& & rho & ICC & $\boldsymbol{P}$ & & rho & ICC & $\boldsymbol{P}$ \\
\hline Peak & & 0.720 & 0.685 & $<0.001$ & & 0.619 & 0.558 & $<0.001$ \\
Current & & 0.712 & 0.718 & $<0.001$ & & 0.600 & 0.631 & $<0.001$ \\
Post & & 0.609 & 0.634 & $<0.001$ & & 0.551 & 0.525 & $<0.001$ \\
\hline 2b & \multicolumn{3}{c}{ NIH (\%PRA) } & & & TCF (\%PRA) \\
& $\mathbf{n}$ & rho & ICC & $\boldsymbol{P}$ & n & rho & ICC & $\boldsymbol{P}$ \\
\hline Peak & 137 & 0.741 & 0.728 & $<0.001$ & 114 & 0.663 & 0.653 & $<0.001$ \\
Current & 139 & 0.706 & 0.750 & $<0.001$ & 116 & 0.566 & 0.618 & $<0.001$ \\
Post & 133 & 0.661 & 0.734 & $<0.001$ & 102 & 0.584 & 0.635 & $<0.001$ \\
\hline
\end{tabular}

rho $=$ Spearman's rho correlation coefficient.

$I C C=$ Intraclass correlation coefficient, measure of method agreement.

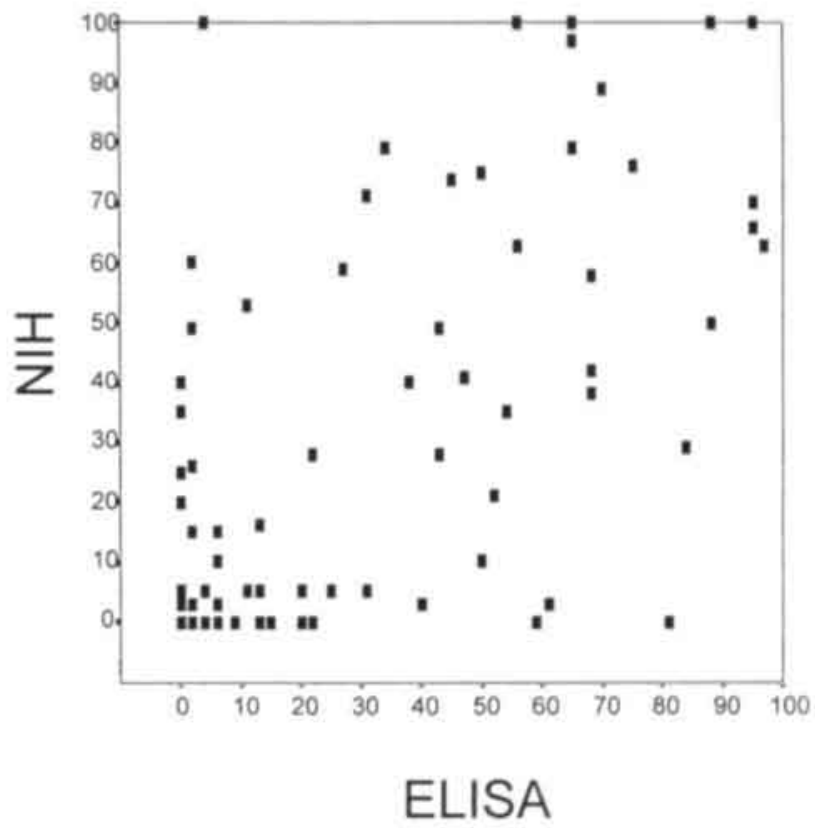

Figure 3.1 Dotplot of \%PRA by ELISA and by NIH for current serum samples from 143 renal transplant patients.

Concordance between ELISA and NIH, and between ELISA and TCF is shown in Table 3.3. The percentages of ELISApos/NIHneg (Epos/Nneg) sera were $8 \%, 9 \%$, and $8 \%$ for peak, current, and post-transplant serum, respectively, while for 
Eneg/Npos $11 \%, 8 \%$, and $14 \%$ were found. In the comparison ELISA/TCF more sera were found in the ELISAneg/TCFpos (Eneg/Tpos) group (22\%, 17\%, 27\%) than in the Epos/Tneg group (5\%,6\%, 4\%). Discordance between ELISA and NIH was detected in $20 \%$ of all sera, for ELISA and TCF in $27 \%$.

Table 3.3 Concordance for antibody detection between ELISA and NIH/TCF in peak, current, and post-transplant sera of 143 renal patients.

\begin{tabular}{lcccc}
\hline & $+1+$ & $+/-$ & $-1+$ & $-/-$ \\
& $\mathbf{n}$ & $\mathbf{n}$ & $\mathbf{n}$ & $\mathbf{n}$ \\
\hline ELISA/NIH & & & & \\
Peak & $44(31 \%)$ & $12(8 \%)$ & $16(11 \%)$ & $71(50 \%)$ \\
Current & $33(23 \%)$ & $13(9 \%)$ & $11(8 \%)$ & $86(60 \%)$ \\
Post-transplant & $40(28 \%)$ & $12(8 \%)$ & $20(14 \%)$ & $71(50 \%)$ \\
ELISA/TCF & & & & \\
Peak & $49(34 \%)$ & $7(5 \%)$ & $31(22 \%)$ & $56(39 \%)$ \\
Current & $37(26 \%)$ & $9(6 \%)$ & $24(17 \%)$ & $73(51 \%)$ \\
Post-transplant & $47(33 \%)$ & $5(4 \%)$ & $38(27 \%)$ & $53(37 \%)$ \\
\hline
\end{tabular}

\section{HLA specificities}

Broad HLA specificities for class 1 and II were determined by ELISA and CDC (NIH/TCF). According to the local transplant protocol, no kidneys were transplanted against which HLA antibodies had been demonstrated by CDC in any pretransplant recipient serum. However, when an HLA antibody specificity had been demonstrated directed against a 'split' antigen, donor kidneys sharing 'broad' HLA antigens but representing the alternative 'split' antigen were accepted. That explains why, in one patient, antibodies with anti-donor specificity were demonstrated by CDC in peak and current serum. The number of patients with HLA specificities was recorded, as was the number of sera with donor-directed specificities. The results of the analysis are shown in Table 3.4. The number of patients with HLA-class I specificities detected by CDC and ELISA were comparable. For donor-directed specificities, the number of patients was too small. The number of patients with HLA class II specificities was higher by ELISA than by TCF $(P<0.01)$. As for donor-directed antibodies, ELISA detected more HLA class I and II specificities than CDC. Since patients were selected for transplantation based on the CDC results for peak and current sera, the comparison between CDC and ELISA for these sera is not valid. For post-transplant sera, the percentage of donor-directed and non-donor-directed specificities detected in ELISA and CDC did not differ. 
Table 3.4 HLA-class I and II specificities in peak, current, and post-transplant sera detected by ELISA and CDC.

\begin{tabular}{lcccc}
\hline & \multicolumn{2}{c}{ Class I } & \multicolumn{2}{c}{ Class II } \\
& Total & Donor-directed & Total & Donor-directed \\
\hline Peak & & & & \\
ELISA & 31 & 4 & 5 & 6 \\
CDC & 36 & 1 & 25 & 0 \\
Current & 26 & 5 & 2 & 7 \\
ELISA & 35 & $1 \cdot$ & 19 & 0 \\
CDC & 29 & 1 & 4 & 8 \\
Post-transplant & 27 & 1 & & 2 \\
ELISA & & & & \\
CDC & & & & \\
\hline
\end{tabular}

- One patient showed anti-A29(19) antibodies in peak and current sera while the donor carried the A30(19) antigen (thus donor-directed on broad, not on split level); the post-transplant serum of this patient was $100 \%$ positive and no specificities could be assigned.

The presence or absence of HLA specificities in concordant and discordant sera was studied. No significant differences between ELISA, NIH, and TCF were noticed in peak and current sera. In post-transplant sera, HLA specificities were detected in 10/12 (83\%) of Epos/Nneg sera versus 7/20 (35\%) of Eneg/Npos sera $(P=0.02)$. For Epos/Tneg, $5 / 5(100 \%)$ sera showed HLA specificity versus $6 / 38$ $(16 \%)$ Eneg/Tpos $(P=0.0004)$. In patients with concordant results in both assays, no difference in the number of sera with HLA specificities was shown; only posttransplant ELISA determined more sera with specificities.

\section{Relationship between screening assays and clinical outcome}

Patients were analyzed grouped according to antibody positivity with peak, current, and post-transplant serum, combined antibody status of current and post-transplant serum, and as concordant/discordant for ELISA/NIH and ELISA/TCF.

\section{Graft survival}

To analyze the additional value of an assay it is necessary to take into account all significant risk factors. Significant risk factors in the basic model of graft survival were gender and donor age $(-2 \mathrm{LL}$ chi-square $=9.05,2 \mathrm{df}, P=0.011)$. There was no interaction between the two factors. None of the assays alone contributed significantly to graft survival (median follow-up 5.29 years), not even when introduced into this model. Reanalysis of the data after exclusion of autocrossmatch-positive sera did not alter the results (results not shown).

\section{Rejection}

Fifty-seven patients were treated for rejection. A renal core biopsy was performed 
in 40 of these patients. In 36 cases, the diagnosis of rejection was confirmed histologically, while 3 biopsies showed no signs of rejection and 1 biopsy was inadequate for examination. In the analyses presented, all 57 patients treated for rejection were included as rejectors. The results of the analyses with rejection defined as biopsy-proven rejection $(\mathrm{n}=36)$ showed very similar results.

Details of the analysis of rejection-free survival for peak, current, and posttransplant sera of 143 patients are shown in Table 3.5. For peak sera, antibody positivity did not contribute significantly to rejection in any assay. In current sera, the results of ELISA as well as of NIH were significant risk factors for rejection, while TCF was not. For post-transplant sera, all assays were significantly correlated with rejection. The odds ratio for antibody positivity varied between 2.12 (ELISA) and $2.75(\mathrm{NIH})(P<0.001$ and $P=0.004)$.

Table 3.5 Cox regression analysis of rejection-free survival of 143 renal transplant recipients. Antibody positivity in peak, current, and post-transplant serum is introduced as a single risk factor and in addition to the basic model".

\begin{tabular}{llcccccc}
\hline & & \multicolumn{4}{c}{ Assay as single risk factor } & \multicolumn{3}{c}{ Assay in addition to basic model } \\
& Assay & $\boldsymbol{P}$ & OR & $\mathbf{9 5 \% - C I}$ & $\boldsymbol{P}$ & OR & $\mathbf{9 5 \% - C I}$ \\
\hline \multirow{2}{*}{ Peak } & ELISA & 0.059 & 1.64 & $(0.98-2.77)$ & 0.308 & 1.37 & $(0.75-2.50)$ \\
& NIH & 0.068 & 1.61 & $(0.96-2.71)$ & 0.398 & 1.30 & $(0.71-2.35)$ \\
\multirow{4}{*}{ Current } & TCF & 0.310 & 1.31 & $(0.77-2.22)$ & 0.696 & 0.89 & $(0.49-1.61)$ \\
& ELISA & 0.002 & 2.21 & $(1.31-3.73)$ & 0.042 & 1.87 & $(1.03-3.40)$ \\
& NIH & 0.013 & 1.93 & $(1.14-3.28)$ & 0.106 & 1.63 & $(0.91-2.92)$ \\
& TCF & 0.098 & 1.55 & $(0.92-2.60)$ & 0.365 & 1.30 & $(0.74-2.31)$ \\
& ELISA & 0.004 & 2.12 & $(1.26-3.57)$ & 0.006 & 2.27 & $(1.27-4.02)$ \\
& NIH & 0.000 & 2.75 & $(1.62-4.68)$ & 0.011 & 2.06 & $(1.18-3.61)$ \\
& TCF & 0.002 & 2.49 & $(1.38-4.49)$ & 0.005 & 2.26 & $(1.24-4.11)$ \\
\hline
\end{tabular}

- The basic model contains the well-known significant risk factors: number of mismatches for HLA-DR, gender, and transplant number (2LL chi-square: 24.356 , df: $6, P=0.0004$ ).

Significant risk factors in the basic model of rejection were gender, transplant number, and number of HLA-DR mismatches $(-2 \mathrm{LL}$ chi-square $=24.356,6 \mathrm{df}$, $P=0.0004)$. The analysis showed that, for peak sera, none of the assays tested contributed significantly to rejection if introduced in the basic model. For current sera, only ELISA showed a significant contribution (partial odds ratio 1.87, $P=0.04$ ). For post-transplant sera, all assays contributed significantly. Reanalysis of the data after exclusion of autocrossmatch-positive sera did not alter the results very much (results not shown).

Analysis of the difference in \%PRA in post-transplant and current serum ( $\triangle \mathrm{PRA})$ showed that $\triangle$ PRA was a risk factor for rejection by NIH $(P=0.006)$ but not by 
ELISA $(P=0.96)$ and TCF $(P=0.08)$. Patients were grouped by $\triangle$ PRA from current to posttransplant serum into three groups according to Monteiro $^{12}:$ (1) decrease $>10 \%,(2)$ indeterminate: between $10 \%$ decrease and $10 \%$ increase, and (3) increase $>10 \%$. Compared with group 2, both group 1 and group 3 had an increased risk of rejection by ELISA (OR 2.47 and 2.06), while by NIH group I had a decreased risk (OR 0.84 ) and group 3 had an increased risk (OR 2.61). When the \% current PRA was already in the model, only the difference in PRA by NIH was a significant risk factor $(P=0.002)$.

Comparison of negative/positive antibody status of current and post-transplant serum was analyzed. Patients were classified into four groups, combining the results of current and post-transplant serum: neg/neg, neg/pos, pos/neg, and pos/pos. Results are shown in Figure 3.2. Cox regression analysis revealed that combined antibody status is a significant risk factor for rejection in ELISA $(P=0.008)$, NIH $(P=0.001)$, and TCF $(P=0.018)$. The odds ratio for the pos/pos group compared to the neg/neg group was 2.6 (95\% Cl: 1.4-4.7) for ELISA, 2.8 (95\% CI: $1.6-5.1)$ for NIH and $2.6(95 \% \mathrm{CI}: 1.3-5.0)$ for TCF. The odds ratio for the neg/pos group compared to the neg/neg group was $2.4(95 \% \mathrm{Cl}: 1.2-4.9)$ for $\mathrm{NIH}$ and 2.3 (95\% CI: 1.1-4.7) for TCF. ELISA neg/pos patients did not have a higher risk for rejection than neg/neg patients. The differences between the other groups were not statistically significant. When combined antibody status of current and post-transplant serum was introduced into a model already containing the wellknown risk factors for rejection (number of DR mismatches, gender, and transplant number), only TCF contributed to rejection $(P=0.025)$. Lack of significance for the other assays might, however, have been due to low patient numbers in the different groups.

Rejection-free survival was also analyzed while patients were classified according to concordant or discordant results in ELISA and NIH/TCF. The graphs of these analyses are shown in Figure 3.3. Patients grouped according to results of peak serum did not differ significantly with regard to rejection, whereas patients grouped according to results of current serum did for both ELISA/NIH $(P=0.002)$ and ELISA/TCF $(P=0.004)$. The odds ratio for concordant positive patients compared to concordant negative patients was 2.8 for ELISA/NIH $(P=0.0005)$ and 2.4 for ELISA/TCF $(P=0.002)$. Both for ELISA/NIH and for ELISA/TCF, patients with neg/pos current sera had a lower rejection risk than patients positive for both assays, while pos/neg patients had a rejection rate that did not differ significantly from patients with concordant results.

For ELISA/NIH in post-transplant sera, neg/pos patients had a rejection rate comparable to that of pos/pos patients (OR: 2.8 and 3.2), while pos/neg patients 
had an intermediate rejection rate between neg/neg and pos/pos patients. Comparable results were found for ELISA and TCF.
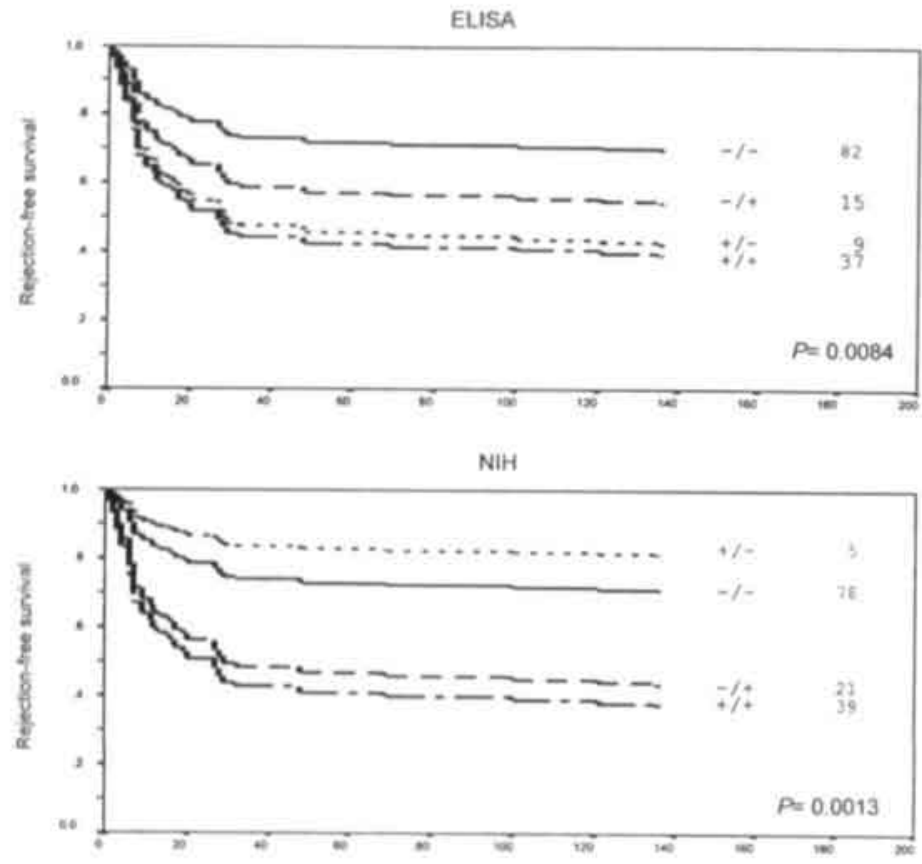

TCF

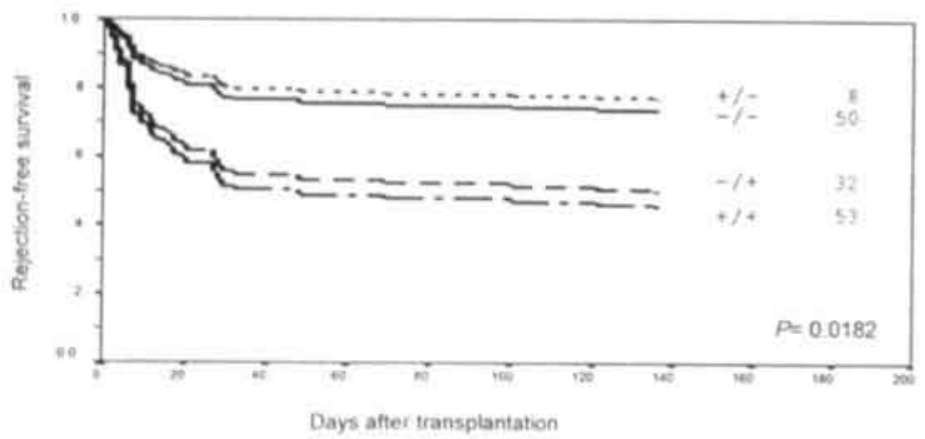

Figure 3.2 Rejection-free survival in 143 renal transplant recipients, grouped according to combined antibody status of current and post-transplant serum. Cox regression model plots by combined antibody status for ELISA. NIH, and TCF. The number of patients in each group is indicated, as is the overall $P$-value of the risk factor. 

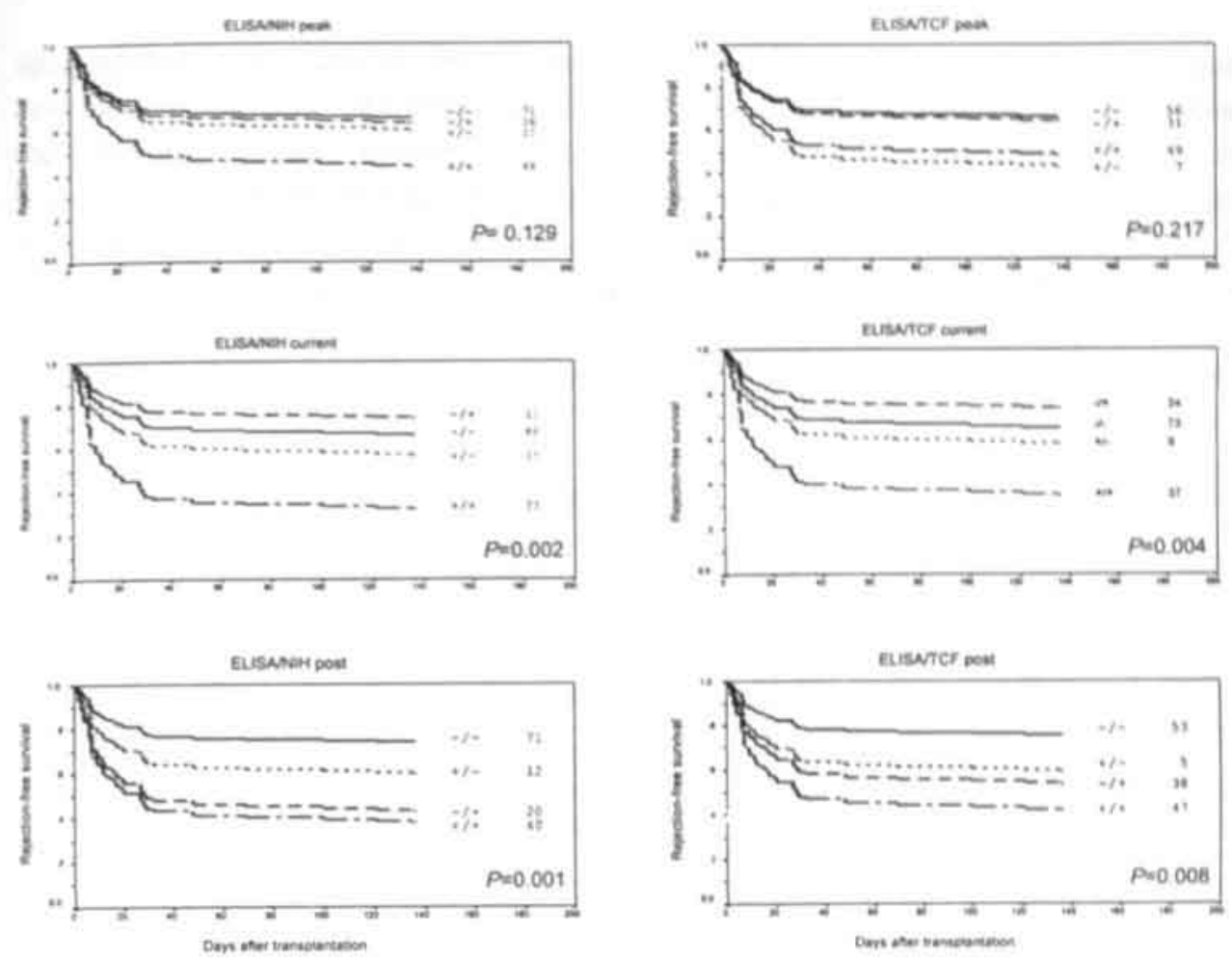

Figure 3.3 Rejection-free survival based on ELISA, NIH, and TCF results with peak, current, and post-transplant serum in 143 renal transplant recipients. Cox regression model plots by concordance/discordance groups for ELISA/NIH (left) and ELISA/TCF (right) with peak (top), current (middle), and post-transplant serum (bottom). The number of patients in each concordance/discordance group is indicated, as is the overall $P$-value of the risk factor.

\section{HLA specificities and clinical outcome}

There was no significant difference in graft survival between patients with and without HLA specificities in peak, current, or post-transplant serum. This held for class I and class II specificities.

To analyze rejection, patients were classified into three groups according to the results of the screening assay. The groups consisted of (1) antibody-negative patients, (2) antibody-positive patients without clearcut HLA specificities in the serum, and (3) antibody-positive patients with assigned HLA specificity in the serum. The incidence of rejection and median PRA for these groups are shown in Table 3.6 for HLA class I and class II specificities. Classified according to class I specificity, patients in group 3 had a lower median PRA than patients in group 2 . This difference was statistically significant for peak, current, and post-transplant 
serum by ELISA ( $P=0.008, P=0.001$, and $P=0.009$, respectively), and by peak and post-transplant serum by NIH $(P=0.004$ and $P=0.001)$, while the current serum showed a tendency towards significance $(P=0.066)$. When patients were classified according to class II specificity, the median PRA between groups 2 and 3 was not significantly different for either ELISA $(P=0.356-0.869)$ or TCF $(P=0.199-0.911)$.

Table 3.6 Incidence of rejection in patients classified according to antibody-positivity and HLA-class I (6a) or II (6b) specificity in peak, current, and post-transplant serum by CDC and ELISA.

\begin{tabular}{|c|c|c|c|c|c|c|c|c|}
\hline & \multicolumn{2}{|c|}{ Ab neg } & \multicolumn{3}{|c|}{ Ab pos / Spec neg } & \multicolumn{3}{|c|}{ Ab pos / Spec pos } \\
\hline & $\mathbf{n}$ & rej\% & $\mathbf{n}$ & rej\% & \%PRA & $\mathbf{n}$ & rej\% & \%PRA \\
\hline \multicolumn{9}{|l|}{$6 a$} \\
\hline \multicolumn{9}{|l|}{ ELISA } \\
\hline Peak & 87 & 35 & 28 & 32 & 69 & 28 & 64 & 43 \\
\hline Current & 97 & 32 & 22 & 41 & 68 & 24 & 71 & 39 \\
\hline Post & 91 & 33 & 26 & 50 & 68 & 26 & 54 & 42 \\
\hline \multicolumn{9}{|l|}{ NIH } \\
\hline Peak & 83 & 35 & 27 & 41 & 85 & 33 & 52 & 40 \\
\hline Current & 99 & 34 & 18 & 61 & 68 & 26 & 46 & 46 \\
\hline Post & 83 & 28 & 34 & 59 & 83 & 26 & 54 & 43 \\
\hline \multicolumn{9}{|l|}{$6 \mathrm{~b}$} \\
\hline \multicolumn{9}{|l|}{ ELISA } \\
\hline Peak & 87 & 35 & 35 & 40 & 61 & 21 & 62 & 45 \\
\hline Current & 97 & 32 & 23 & 52 & 50 & 23 & 61 & 50 \\
\hline Post & 91 & 33 & 33 & 46 & 50 & 19 & 63 & 56 \\
\hline \multicolumn{9}{|l|}{ TCF } \\
\hline Peak & 63 & 37 & 75 & 43 & 85 & 5 & 40 & 90 \\
\hline Current & 82 & 35 & 59 & 46 & 70 & 2 & 50 & 92 \\
\hline Post & 58 & 26 & 80 & 49 & 90 & 4 & 50 & 84 \\
\hline
\end{tabular}

Detection of antibodies with HLA class I specificity by ELISA resulted in an increase in rejection rate compared to antibody-positive patients without specificity, regardless of the lower PRA. The rejection rate was significantly different between the groups for peak and current sera $(P=0.013$ and $P=0.0002)$ in contrast to the post-transplant serum $(P=0.08)$. Detection of antibodies with HLA-class I specificity by NIH did not result in such an increase in rejection. Differences in rejection rate were not statistically significant in peak or current sera $(P=0.27$ and $P=0.08$ ). In post-transplant sera, the highest rejection incidence was found in the antibody-positive group with no assigned HLA specificities and the highest \%PRA.

Classifying patients according to detection of class II antibodies by ELISA showed 
that patients with HLA specificities had a higher incidence of rejection than patients without specificities. Differences in rejection rate were statistically significant between patients grouped according to the result of current $(P<0.02)$ and posttransplant serum $(P<0.04)$. The numbers of patients with specificity by TCF were too small to analyze properly. Antibody positivity by TCF did not have an effect on rejection in pretransplant sera, whereas it did with post-transplant serum $(P<0.02)$.

\section{Discussion}

Originally, the commercial ELISA assay, Prastat, was launched as a screening technique for the detection of anti-HLA class I antibodies of the IgG type. Therefore, the first reports compared Prastat to such class I screening techniques as NIH and AHG. Recently, it was shown that the soluble HLA antigens trapped on the trays were class I as well as class II. Thus, both anti-HLA class I and II IgG antibodies are detected. In the present study, we compared the results obtained with the ELISA technique with those of both NIH and TCF, and we also studied the relationship between the screening assay results and clinical outcome.

\section{Method agreement}

The numbers of antibody-positive patients as well as the mean PRA were similar with ELISA and NIH. However, the method agreement and rank correlations were shown to be insufficient. The correlations between ELISA and NIH (rho= $0.609-0.720$ ) were slightly better than those reported previously, the latter ranging from 0.4 to $0.6^{7.8,11,12}$. When the influence of autoantibodies was eliminated, no improvement in the correlations could be shown ( $r$ ho $=0.661-0.741)$. This contrasts with the findings of Monteiro, who showed an increase from 0.4 to 0.6 , and of Zachary, who observed an increase from 0.56 to 0.78 , after exclusion of autoantibodies ${ }^{11,12}$.

Because ELISA and TCF both detect HLA class I and II antibodies, method agreement was expected to be better when ELISA was compared with TCF. Method agreement for \%PRA between ELISA and TCF (ICC: 0.525-0.631) was, however, lower than between ELISA and NIH (ICC: 0.634-0.718). Exclusion of sera with autoantibodies did not improve the ICC. A higher \%PRA was obtained in the TCF assay. The TCF technique is very sensitive and detects quite some "background noise". These usually weak reactions are considered false-positive and, for that reason, the cut-off point of the test is set at $25 \%$ compared to $10 \%$ with the other techniques. Nevertheless, more patients remained antibody-positive 
in TCF compared to ELISA. In that respect, it should be noted that the ELISA kit was intended to detect only class I antibodies, and the panel, therefore, was not selected or standardized for class II antigens ${ }^{5}$.

Discordance between ELISA and NIH/TCF was $20 \%$ and $27 \%$. For NIH, this percentage is comparable to those reported previously, while for TCF the percentage is higher than reported ${ }^{9.5 .20}$. Class II screening results were reported by Worthington using CLL-lines as target cells, whereas in this study unseparated peripheral blood lymphocytes were used ${ }^{5}$. More sera were NIH- or TCF-positive and ELISA-negative than vice versa. Patients who were antibody-positive by one assay and antibody-negative by the other assay usually had a lower \%PRA than patients who were antibody-positive by both assays. This discordance might at least partially be explained by false-positive reactions or low titers of antibodies. HLA class I specificities were detected in comparable numbers with ELISA and CDC, while HLA class II specificities were more frequently detected by ELISA, which might be due to false-positive reactions of TCF.

We conclude that, in this patient population, the method agreement between ELISA and NIH or TCF is too low to allow one to replace one test by the other, and that this cannot be attributed to the presence of autoantibodies or HLA class II antibodies, as has been suggested by Schönemann ${ }^{20}$.

\section{ELISA and clinical outcome}

To ascertain the clinical value of the various assays, we have to investigate the relationship of results with rejection and graft survival. Especially, when the method agreement is insufficient, differences in correlation with clinical outcome may indicate a preference of one assay over the other.

Rejection and graft survival are determined by many risk factors, and these may be related to PRA. Therefore, a multivariate analysis is mandatory. None of the assays contributed significantly to graft survival up to 5 years, either directly (as a single risk factor in the model) or in a multifactorial analysis. Excluding positive sera containing autoantibodies from the analysis did not change the results. These findings confirm the conclusions of Dalla Vecchia and Neylan ${ }^{9.19}$. They are at variance with the results of Kerman et al. ${ }^{10}$, who found $11 \%-14 \%$ lower graft survival in a large patient population with a pretransplant ELISA-positive serum. A difference in graft survival of $11 \%-14 \%$ cannot be detected with the number of patients in our study.

Although there was no influence on graft survival, screening results did contribute significantly to the occurrence of acute rejection. Results were analyzed when 
grouped in three ways: (1) according to antibody positivity with peak, current, and posttransplant serum, (2) for combined antibody status of current and posttransplant serum, and (3) as concordant/discordant for ELISA/NIH and ELISA/TCF. Previous studies on the relationship between ELISA and rejection used univariate analyses ${ }^{5,7-10.12 .13 .19 .20}$, while in this study both uni- and multivariate analyses were performed.

Positivity of current serum by ELISA and NIH was a risk factor for rejection. As might be expected, the odds ratios were lower when antibody positivity was introduced into the basic model already containing the other significant risk factors. Antibody positivity by ELISA remained significant, while antibody positivity by NIH did not. Because the difference between ELISA and NIH was not statistically significant, both assays probably have an equal predictive value for rejection. These findings are a confirmation and extension of the findings by Dalla Vecchia and Kerman ${ }^{9.10 .13}$. Yet, in contrast to our findings, these authors found no similar result with the $\mathrm{CDC}$ assay in univariate analysis.

It has been reported that the presence of HLA antibodies is correlated with the occurrence of vascular rejection. Usually this type of rejection presents as steroidresistant rejection ${ }^{21.22}$. Because rejection treatment consisted of rATG, we were unable to analyze our data to ascertain whether antibody-positive patients had a higher incidence of steroid-resistant rejection.

The difference in \%PRA between current and post-transplant serum ( $\triangle P R A)$ was analyzed in a univariate way. $\triangle \mathrm{PRA}$ was a risk factor for rejection only with NIH. A rise in PRA $>10 \%$ post-transplant by both ELISA and NIH was a risk factor for rejection. This is in contrast to the findings of Monteiro ${ }^{12}$, who did not find an association between rise in PRA $>10 \%$ by NIH and rejection.

Combined antibody status of current and post-transplant serum was a significant risk factor for all assays (odds ratio 2.6-2.8) in univariate analysis, but only for TCF in multivariate analysis. In the other assays, the lack of statistical significance might have been caused by the relatively small sample size. The combined antibody status defined by NIH and TCF were risk factors of the same magnitude as by ELISA in the univariate analysis.

To investigate whether the combination of results of ELISA and NIH or TCF per serum could discriminate the group of patients at an increased risk for rejection, patients were classified according to concordance of sera for ELISA and NIH, or for ELISA and TCF. Patients reacting pos/pos in current serum had an increased risk for rejection compared to neg/neg patients (rejection-free survival $30 \%$ versus $70 \%$ ). This held for both ELISA/NIH and ELISA/TCF. Thus, concordant results in current serum have a strong clinical impact on subsequent transplantation, while discordant reactions in current serum do not influence rejection-free survival. This 
may imply that discordant reactions are due to false-positive reactions or clinically less relevant antibodies.

In contrast to the results of current serum, analysis of post-transplant serum showed that patients with discordant ELISAneg/NIHpos or ELISAneg/TCFpos sera were at an increased risk for rejection. They had a rejection-free survival of $40 \%$, comparable to that of pos/pos patients. The positive NIH reactions could not be explained by the presence of rATG in the serum of rejectors because the presence of rATG in serum, in contrast to OKT3, does not result in a positive NIH-assay ${ }^{17}$.

The most important aspect of HLA screening of patient sera is the determination of the HLA specificity of the antibodies. In our center, HLA antigens were not considered acceptable for a patient if antibodies against the given specificity had been demonstrated either at the time of transplantation or in the past. Thus, antibody positivity by TCF and/or NIH in peak and current serum do not indicate donor-directed activity. Detection by ELISA of HLA class I antibodies in peak and current serum was correlated with a high incidence of rejection $(67 \%)$, even when the antibodies were not donor-directed $(63 \%)$, but not with graft loss. This suggests that it reflects a general state of sensitization. However, when no HLA-specific antibodies could be defined in ELISA-positive sera, there was no increase in acute rejections.

Detection of HLA class II antibodies by ELISA and TCF often coincided with detection of HLA class I specificity. The number of patients with only HLA class II antibodies was too small to be analyzed as a subgroup. By ELISA many more patients were considered to have anti-HLA class II antibodies than by TCF. Because the rejection rate of these patients hardly differed from that of antibodypositive patients without HLA class II specificity, their clinical significance seems to be minor.

In conclusion, ELISA and CDC present themselves as complementary screening techniques in this study. An optimal strategy for combining these techniques needs to be determined, also taking into account cost-effectiveness. The ELISA kit is rather expensive; on the other hand, the CDC assay is more time-consuming. An important issue to be addressed is the combination of screening and final crossmatch technique. Antibodies detected in screening by one assay may not be detected in the crossmatch performed in another technique. In present practice, this will often be the case, since most centers use $C D C$ as the final crossmatch technique. Yet, an ELISA-based HLA class I crossmatch kit has been introduced, making it possible to compare results obtained in one technique with clinical outcome. More information on this issue could be provided by a prospective, randomized trial comparing patients screened and crossmatched by ELISA or CDC. 


\section{References}

1. Chapman JR, Taylor CJ. Ting A, Morris PJ. Immunoglobulin class and specificity of antibodies causing positive $\mathrm{T}$ cell crossmatches. Relationship to renal transplant outcome. Transplantation 1986; 42: 608-13.

2. Taylor CJ, Chapman JR, Ting A, Morris PJ. Characterization of lymphocytotoxic antibodies causing a positive crossmatch in renal transplantation. Transplantation 1989; 48: 953-8.

3. Cardella CJ, Falk IA, Nicholson MH, Harding M. Cook GT. Successful renal transplantation in patients with T-cell reactivity to donor. Lancet 1982;2: 1240-3.

4. Ting A. The lymphocytotoxic crossmatch test in clinical renal transplantation. Transplantation 1983; 35: 403-7.

5. Worthington JE, Thomas AA, Dyer PA, Martin S. Detection of HLA-specific antibodies by PRA-STAT and their association with transplant outcome. Transplantation 1998; 65: 121-5.

6. Ettenger RB, Jordan S, Fine RN. Autolymphocytotoxic antibodies in patients on dialysis awaiting renal transplantation. Transplantation 1981: $32: 248-51$.

7. Harmer AW, Heads AJ, Vaugha RW. Detection of HLA Class I- and Class IIspecific antibodies by flow cytometry and PRA-STAT screening in renal transplant recipients. Transplantation 1997; 63: 1828-32.

8. Regan J, Monteiro F, Speiser D, Kalil J, Pouletty P, Buelow R. Pretransplant rejection risk assessment through Enzyme-Linked Immunosorbent Assay analysis of anti-HLA Class I antibodies. Am J Kidney Dis 1996; 28; 92-8.

9. Dalla Vecchia LK, Book BK, Milgrom ML, Jindal RM, Leapman SB, Filo RS, Pescovitz MD. Predictive value of enzyme-linked immunosorbent assay-detected IgG anti-HLA antibodies for pediatric renal allograft rejection. Transplantation 1997; $64: 1744-7$.

10. Kerman RH, Susskind B, Buelow R, Regan J, Pouletty P, Williams J, Gerdami K, Kerman DH, Katz SM, Van Buren CT, Kahan BD. Correlation of ELISA-detected $\operatorname{IgG}$ and IgA anti-HLA antibodies in pretransplant sera with renal allograft rejection. Transplantation 1996; 62: 201-5.

11. Zachary AA, Griffin J, Lucas DP, Hart JM, Leffell MS. Evaluation of HLA antibodies with the PRA-STAT test. An ELISA test using soluble HLA class I molecules. Transplantation 1995; 60: 1600-6.

12. Monteiro F, Buelow R, Mineiro C, Rodriques H, Kalil J. Identification of patients at high risk of graft loss by pre- and posttransplant monitoring of anti-HLA class I IgG antibodies by enzyme-linked immunosorbent assay. Transplantation 1997; 63: 542-6.

13. Kerman RH, Orosz CG, Lorber MI. Clinical relevance of anti-HLA antibodies pre and post transplant. Am J Med Sci 1997; 313: 275-8.

14. Christiaans MHL, Overhof R, ten Haaft A, Nieman F, van Hooff JP, van den Berg-Loonen PM. No advantage of FC-XM over CDC in immunologically welldocumented renal allograft recipients. Transplantation 1996; 62: 1341-7. 
15. Christiaans MHL, Overhof-de Roos R, Nieman F, van Hooff JP, van den BergLoonen EM. Donor-specific antibodies after transplantation by flow cytometry. Relative change in fluorescence ratio most sensitive risk factor for graft survival. Transplantation 1998; 65: 427-33.

16. Christiaans MHL, van Hooff JP, Nieman F, van den Berg-Loonen PM. HLA-DR matched transfusions: development of donor-specific T- and B-cell antibodies and renal allograft outcome. Transplantation 1999; 67: 1029-35.

17. van den Berg-Loonen E, Overhof R. Tillemans $M$, van Hooff H. Allo and auto crossmatches after transplantation. Transpl Int 1992; 5 [Suppl 1]: 594-5.

18. Chapter 8 Reliability. In: Steiner DL, Norman GR, eds. Health measurement scales. A practical guide to their development and use. 2nd edition. Oxford University Press, 1995.

19. Neylan J, De Smet W, Stijlemans B, Brinker K, Gonwa T, Moran J, Segers A on behalf of the anti-CD45 study group. Detection of clinically relevant antibodies pretransplant and posttransplant with PRA-STAT. Transplant Proc 1997; 29: 330-2.

20. Schönemann C, Groth J, Leverenz S, May G. HLA class I and class II antibodies. Monitoring before and after kidney transplantation and their clinical relevance. Transplantation 1998; 65: 1519-23.

21. van Saase JLCM, van der Woude FJ, Thorogood J, et al. The relation between acute vascular and interstitial renal allograft rejection and subsequent chronic rejection. Transplantation 1995; 59: 1280-5.

22. Yard B, Spruyt-Gerritse M, Claas F, Thorogood J, Bruijn JA, Paape ME, Stein SY, van Es LA, Van Bockel JH, Kooymans-Coutinho M, Daha MR, van der Woude FJ. The clinical significance of allospecific antibodies against endothelial cells detected with an antibody-dependent cellular cytotoxicity assay for vascular rejection and graft loss after renal transplantation. Transplantation 1993; 55: $1287-93$. 


\section{Chapter 4}

\section{Flow cytometry: principles and crossmatch protocol}




\section{Introduction}

Flow cytometry (FC) crossmatching is reported to be a powerful tool in the area of histocompatibility due to its high sensitivity and specificity in the detection of cellular antigens and preformed antibodies ${ }^{1.3}$. It is an extension of the traditional indirect immunofluorescence technique. Serum of the patient is incubated with cells of the potential donor to allow antibody to bind to the cells. Thereafter, a fluorescein isothiocyanate (FITC)-conjugated anti-human IgG antibody is added that binds to the already attached antibodies on the donor cells. Cell fluorescence is measured in a flow cytometer. FC crossmatches differ from the classical complement-dependent cytotoxicity crossmatches in that they have more objective parameters and a higher sensitivity; however, they also require a larger number of cells for testing. In the next sections, the principles of flow cytometry are described. Flow cytometry is then applied for donor-recipient crossmatching. Finally, the crossmatch protocols used for this thesis are presented.

\section{Principles of flow cytometry}

\section{Principle}

Flow cytometry is a process, whereby multiple characteristics of individual cells or particles are simultaneously analyzed ${ }^{4-7}$. Cells are individually sent through a cytometer. Each cell passes the light source and the interactions of the cell or particles with the light beam are recorded by sensors. The refined light source is most commonly a laser. With the use of sophisticated computer analysis systems, detailed information on structural and functional properties of cells and cell subpopulations, such as size, shape, membrane permeability, and surface receptors, can be obtained. In histocompatibility laboratories, flow cytometers are primarily used for the identification of cell surface markers and for the detection of antibodies reactive with HLA molecules on the cell surface.

\section{Flow cytometer}

The major components of a flow cytometer are shown in Figure 4.1. Under pressure, a cell suspension stained with fluorochrome-labelled antibodies is injected into a sheath stream of buffer. The cells are focused in the center of the sample stream. They become aligned in a single file and pass through the flow cell and into the focused beam of light. The interaction of every cell with the beam of light results in scattering of the light and the emittance of fluorescence. The kind of fluorescence emitted is dependent on the light source available and the fluorochrome used. The scattered light and fluorescence signals of each cell are 
recorded by different photomultiplier tubes (PMTs). After conversion into an electronic signal, they are transferred to the computer for data analysis. For most instruments, either selected events ('gated' data) or all events (list mode) may be stored for subsequent analysis.

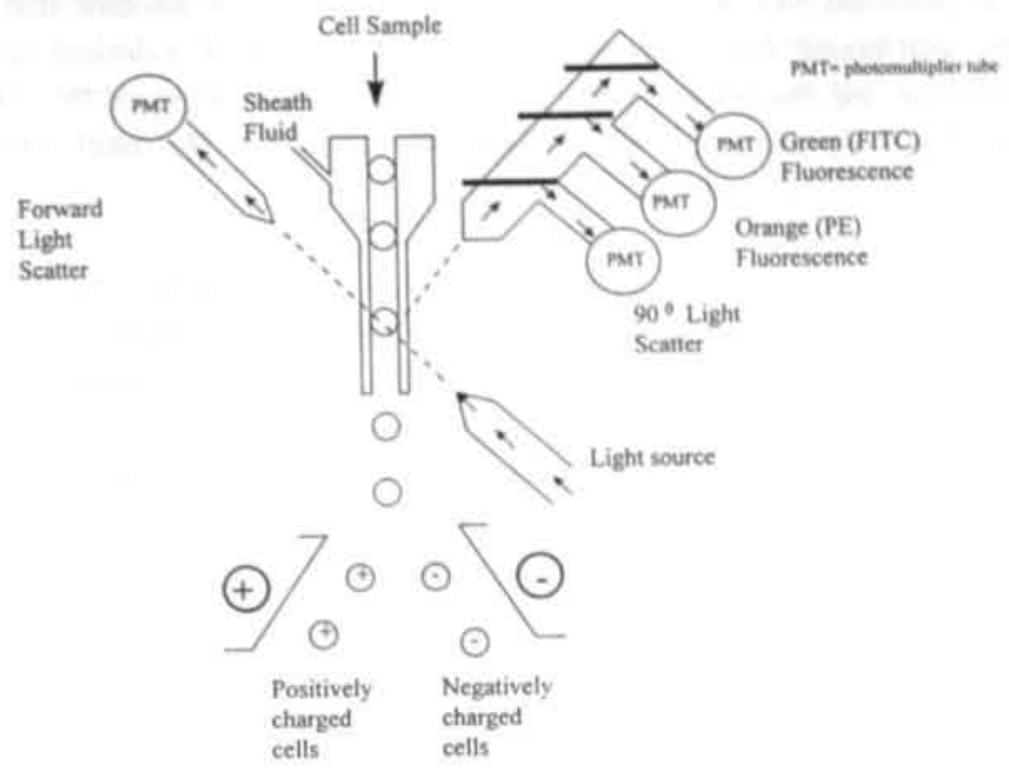

Figure 4.1 Main components of a flow cytometer

\section{Laser}

The light source is a critical component of the cytometer. The common light source used to be an argon laser that produced a blue-green 488 -nm beam suitable for exciting fluorochromes such as phycoerythrin and fluorescein isothiocyanate. Currently, lasers are used that emit 15-25 milliwatts of energy (compared to 5 watt in the past) and that allow air cooling. The present machines are more compact and less expensive in both initial and maintenance costs. Other lasers, such as heliumneon (633-nm excitation) or helium-cadmium (325-nm excitation), may be employed in addition to the argon laser, allowing multi-beam measurements.

\section{Scatter parameters}

The most appealing feature of flow cytometry is its ability to simultaneously measure multiple parameters of individual cells at high speed. As can be seen in Figure 4.1, the parameters most commonly used are: (1) forward scatter angle (FSC), (2) side scatter angle (SSC; $90^{\circ}$ light scatter), (3) orange fluorescence and (4) green fluorescence. For flow cytometry crossmatching, these parameters are 
used to analyze cell populations, i.e., lymphocytes with specific characteristics. As the cell is hit by the focused beam of light, some light photons may be absorbed while others are refracted or scattered. The amount of laser light that is scattered in the forward direction is proportional to the cell size, while the amount of laser light that is side-scattered correlates with cell complexity. The photons that are absorbed by the cell-bound fluorochrome are emitted as light of a longer wavelength and detected by the fluorescence detectors that are at an angle of $90^{\circ}$ to the focused beam. Not only these parameters, but also changes in electrical resistance can be measured and correlated with cell volume.

By means of certain characteristics (i.e., FSC, SSC) leukocytes can be differentiated into subpopulations of monocytes, granulocytes, and lymphocytes. Boundaries or 'gates' are set electronically to define a window around these subpopulations (Figure 4.2). This allows analysis of a single cell type out of a mixed cell population. By using appropriate fluorochrome labels, even subsets of cells within a 'gate' can be measured. Mature T lymphocytes, for example, can be stained with a PE-conjugated monoclonal antibody directed against the CD3 complex. After 'gating' the lymphocytes by FSS and SSC, this subset can be detected by their orange fluorescence.

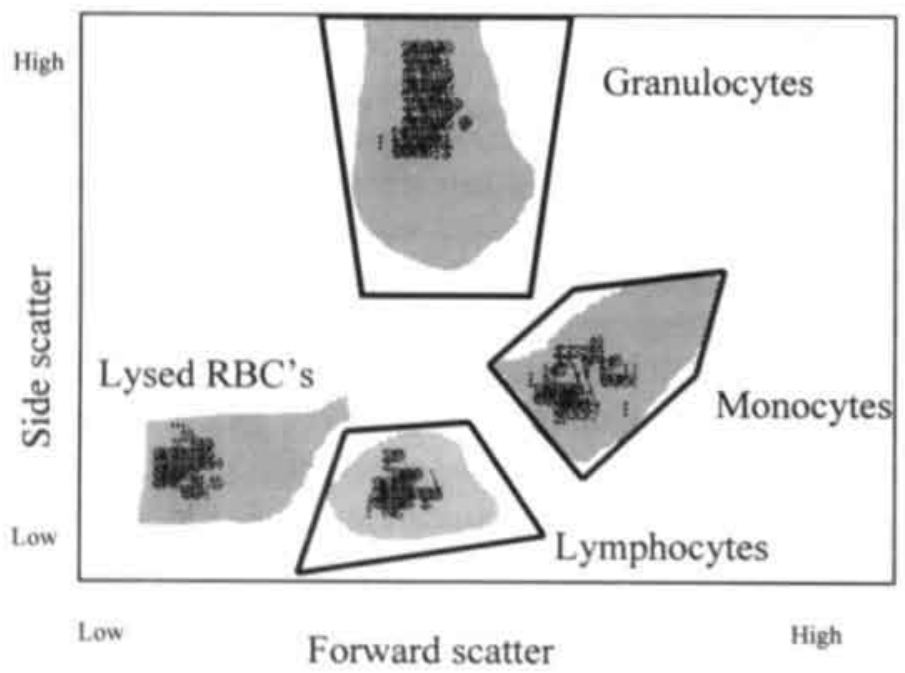

Figure 4.2 Schematic example of identification of 3 distinct (gated) populations based on light scatter.

\section{Photomultiplier}

The refracted light or fluorescent emission arrives at the PMT and is converted into electric current with an amplitude correlating with the intensity of the light signal. 
The individual pulses are recorded on an electronic scale that is divided into voltage increments or channels. In addition to recording the light, the PMT also amplifies the signal output. The output can either be directly proportional to the fluorescence emission (linear) or be expressed as a percentual increase in fluorescence intensity corresponding to a constant increase in channel number (logarithmic). The latter is generally used to study subsets of cells varying widely in fluorescence intensity in order to display all populations on the same scale. Figure 4.3 is an example of a single parameter histogram.

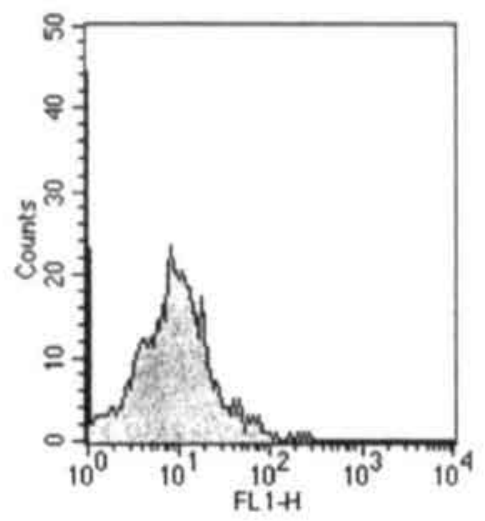

Figure 4.3 Single parameter histogram. The relative fluorescence intensity is displayed on the $\mathrm{X}$-axis and the frequency of events (the number of cells) on the Y-axis.

\section{Fluorescence intensity}

The fluorescence intensity is often designated by a channel number ( 0 to 256 or 0 to 1023) and most instruments define the mean, median, and peak channel of fluorescence intensity for a given cell population. The mean and median channel of fluorescence are used as qualitative measures of antigen density. Increased antigen density results in increased fluorescence intensity that is indicated by a shift to higher channel values. Mean or median channel of fluorescence is also used in some FC crossmatch techniques as the criterion for determining a positive reaction.

\section{Two-color fluorescence}

To differentiate subpopulations of cells, two- or three-color fluorescence analysis is needed. The optimal fluorochromes have approximately the same maximum excitation wavelength, while their emission spectra are sufficiently different to allow for their signals to be easily detected separately.

For two-color analysis, the combination of FITC and PE is widely used. Although excited by a single light source of $488 \mathrm{~nm}$ (argon laser), they emit their radiation at 
different wavelengths ( $517 \mathrm{~nm}$ green and $578 \mathrm{~nm}$ orange, respectively). For threecolor analysis, a second laser is needed with a different wavelength to excite the third fluorochrome (e.g., rhodamine). Essential for both types of analysis are the precise alignment and calibration of lasers and optics, particularly when two lasers are used.

With two-color analysis, it is possible to analyze the B- or T-lymphocyte subsets in a sample of leukocytes. With a PE-conjugated anti-CD3 monoclonal antibody, the subset of $\mathrm{T}$ lymphocytes can be analyzed for their binding of alloantibodies. By using an anti-CD19 instead of an anti-CD3 PE-conjugated monoclonal, the subset of B lymphocytes can be analysed. By three colour analysis T and B lymphocytes can be analyzed simultaneously in the same sample.

\section{Quality assurance}

Correct interpretation of results is possible only with proper controls. Alignment of the light source and optical system must be verified routinely. A slight deviation adversely affects the collection of the FSC and SSC as well as the fluorescent excitations. This alignment control is usually performed by analyzing a standard cell or particle suspension, such as fluorescent latex beads or fixed stained cells. Appropriate positive and negative controls have to be used for fluorescent antibody staining. Indirect staining often results in a high level of nonspecific background fluorescence due to Fc-receptor binding. However, dead or dying cells will also nonspecifically absorb fluorescence-conjugated antiglobulins. Control for background staining is essential for interpretation of flow cytometry crossmatches. For two-color analysis positive and negative controls are necessary to establish the correct definition of single and double-stained cell populations.

Some fluorochromes emit light over a range of wavelengths. When staining samples with two or more fluorochromes, one fluorochrome often emits some light at the same wavelength as the other fluorochrome. This phenomenon is called spectral overlap. Thus part of the FITC (FL1) signal falls into the PE (FL2) detector. Filtering with optical filters and electronic fluorescence compensation eliminates most of the overlapping signal. A percentage of the contaminating signal is subtracted from the signal that is measured.

For the FC crossmatches described in this thesis, the quality assurance of the routine instrument function included calibration by test beads (Calibrite Beads, Becton \& Dickinson, California, USA) and fluorescence compensation. 


\section{Flow cytometry crossmatches}

\section{History}

The FC crossmatch was introduced in 1983 by Garovoy et al. as a more sensitive method than the CDC crossmatch for the detection of preformed anti-HLA antibodies in renal transplant recipients. The main advantages reported are increased sensitivity ${ }^{1.3}$, detection of non-complement fixing antibodies ${ }^{8.9}$, and correlation with transplant outcome $\mathrm{1}^{10-14}$. Disadvantages are the large number of cells needed to perform a test, the amount of serum required, and the initial cost of the apparatus.

There are different protocols in use for performing FC crossmatches. They differ in the type of antibody used for measuring cell-bound antibody ${ }^{2,11.13}$, the expression of channel fluorescence $e^{2.11 .13}$, and the definition of positivity ${ }^{2,11.15-17}$. The label used for cell-bound antibody is specific anti-immunoglobulin $\mathrm{G}^{11.13}$ or total antiimmunoglobulin $^{2}$. Fluorescence is expressed as mean ${ }^{2.13}$ or median channel ${ }^{11}$. Positivity is expressed as the number of channel shifts ${ }^{2.11 .15 .16}$ or as the fluorescence ratio of test and control serum ${ }^{17}$. The FC crossmatch is performed for $\mathrm{T}$ cells ${ }^{18}$ or for $\mathrm{T}$ and $\mathrm{B}$ cells $\mathrm{s}^{11.13 .17}$. The serum used for the FC crossmatch is current serum $^{11.13}$, although peak serum may also be included ${ }^{16.17}$. Because of all the different protocols, one must be cautious when comparing these studies. Most of the studies were performed in recipients transplanted with a negative NIH crossmatch irrespective of the FC crossmatch result. In some studies the result of the FC crossmatch influenced the decision to perform the transplantation or the type of immunosuppression used ${ }^{15.17}$.

Cook et al. studied a group of NIH crossmatch-negative recipients of a cadaveric kidney graft ${ }^{10}$. Depending on the FC crossmatch result, recipients were added to the FC-positive or FC-negative group. At 1 month, graft survival was worse for recipients with a positive FC crossmatch than it was for those with a negative FC crossmatch. This held for first transplants (78\% versus $93 \%$ ) as well as for retransplants ( $44 \%$ versus $79 \%$ ). For first transplants, only sensitized recipients (PRA $>10 \%$ ) were shown to have an increased risk. At 3 months after transplantation, better graft survival was reported for FC crossmatch-negative recipients of first transplants $(77.8 \%$ versus $95.8 \%)$ and retransplants $(33.3 \%$ versus $88.2 \%$ ) by the same authors ${ }^{19}$. However, graft survival at 1 year showed no significant difference in first transplants $(70 \%$ versus $75 \%)$; in retransplants, the significant difference remained $(45 \% \text { versus } 65 \%, P=0.05)^{11}$. A large study of 841 first transplants by Ogura and coworkers showed improved 1-year graft survival for 693 FC crossmatch-negative recipients (82\%) compared to 148 FC crossmatch 
positive patients $(75 \% ; P=0.01)^{18}$. Talbot et al. found no significant difference in graft survival at 3 months between FC crossmatch-positive and FC crossmatchnegative transplants if the original NIH crossmatch at the time of transplantation was negative ${ }^{13}$. Kerman et al. showed a significant difference in 1-year graft survival for retransplant patients ( $50 \%$ versus $89 \%$ ). He described the anti-human globulin (AHG) crossmatch as being as sensitive as the FC crossmatch ( $46 \%$ versus $77 \%)^{12}$. A drawback in comparing these studies is that major inclusion and exclusion criteria differed, e.g., the type of donor (living related or cadaveric), exclusion of technical and/or nonimmunological failures, and differences between the FC crossmatch-positive and -negative group in PRA grade or HLA match. All these factors are known to influence graft survival.

Most studies reported fewer acute rejections in FC crossmatch-negative than in FC crossmatch-positive recipients ${ }^{12,13}$. Thistlethwaite et al. found more irreversible rejections in FC crossmatch-positive recipients at 3 months postgrafting. This was true for recipients treated with cyclosporin $\mathrm{A}$ and prednisolone, as well as for recipients treated with azathioprine in addition (triple therapy) ${ }^{14}$. Lazda et al. could not confirm these finding $\mathrm{s}^{20}$.

Other clinical parameters were investigated by Talbot et al. in a retrospective study. FC crossmatch-negative and -positive patients were studied at 3 months after transplantation excluding nonimmunological failures. The FC crossmatch-negative patients had a significantly lower serum creatinine $(152.3$ versus $181.6 \mu \mathrm{mol} / \mathrm{L})$, a shorter duration of primary nonfunction (6.0 versus 16.3 days), and fewer days of hospitalization ( 28.9 versus 45.4 days $)^{13}$. They were not able to confirm these results in a prospective study, although there was a tendency towards a better clinical course for FC crossmatch-negative recipients. The latter showed less graft failure and less need for rejection treatment with antithymocyte globulin ${ }^{21}$.

Assuming the FC crossmatch detects clinically relevant antibodies, the question remains whether these are the same antibodies detected by the NIH crossmatch (complement binding) or additional non-complement-binding antibodies. Talbot et al. studied the isotype of the IgG antibody in the FC crossmatch ${ }^{9}$ and showed the presence of gamma 1, 2, 3, as well as 4 . IgG4 is a non-complement-binding isotype. Karuppan showed postoperative complications to be correlated with a positive FC crossmatch due to non-complement-fixing antibodies ${ }^{22}$ and harmful antibodies to have HLA specificity, particularly class $\mathrm{I}^{23,24}$.

Although FC crossmatching has been performed for more than 10 years now, quality control programms have only recently been started by exchanging sera and cells between different centers. 
The method most often used for FC crossmatch is the two-color fluorescence procedure, which combines the direct and the indirect fluorescence techniques. Cells are incubated with patient serum and antigen-antibody binding is made visible by a FITC-conjugated anti-human IgG antibody (indirect technique), while the $\mathrm{T}$ cells are designated by a PE-conjugated mouse anti-CD3 monoclonal (direct technique). In the following sections, the protocol used at the Tissue Typing Laboratory of the University Hospital Maastricht (azM) for FC crossmatches is described. The protocol was based on the FC crossmatch described by Scornik et al. ${ }^{17}$.

\section{Protocol}

Cells were liquid-nitrogen-stored spleen lymphocytes of the kidney donor and peripheral blood lymphocytes of the recipient. The lymphocytes were purified by Ficoll gradient centrifugation and stored with DMSO as the freeze protecting agent. For patients on the Eurotransplant waiting list, sera were collected every 3 months and stored at $-30^{\circ} \mathrm{C}$. Three sera per patient were tested for FC crossmatch: (1) the peak serum, i.e., the serum with the highest percentage of panel reactivity (for retransplant patients, this was drawn after failure of the previous graft), (2) the current serum, i.e., the serum drawn at the time of transplantation, and (3) the post-transplant serum, i.e., the serum drawn after rejection or, if no rejection episode occurred, within 6 months after transplantation. Serum was drawn 2-4 weeks after removal of a kidney graft. To avoid false-positive results, sera drawn within 10 days after the last administration of OKT3 were excluded ${ }^{25}$.

The same lot of FITC-labelled $\mathrm{F}\left(\mathrm{ab}^{\prime}\right) 2$ anti-human $\mathrm{IgG}$ was used (TAGO, Burlingame, California, USA) for all FC crossmatches performed. Every testing day, fresh stock solution was thawed and microaggregates of immunoglobulins and immune complexes of goat $\mathrm{F}\left(\mathrm{ab}^{\prime}\right) 2$ anti-human $\operatorname{IgG}$ FITC were removed by centrifugation at $3000 \mathrm{rpm}$ for 5 minutes. The anti-IgG was diluted 1:32 and stored in the dark at $4^{\circ} \mathrm{C}$ until use. Mouse monoclonal PE-conjugated anti-human CD3 was used undiluted (Dakopatts a/s, Glostrup, Denmark).

Thawed cells were added to $8 \mathrm{~mL}$ of RPMI- $20 \%$ FCS at $4^{\circ} \mathrm{C}$. After centrifugation (700x g for 5 minutes at $4^{\circ} \mathrm{C}$ ), the supernatant was removed. Eight milliliters of PBS- $1 \%$ BSA was added to the pellet and after centrifugation at $18^{\circ} \mathrm{C}$ the volume was adjusted to $8 \mathrm{~mL}$ with PBS-1\% BSA. The procedure was repeated and finally the cells were resuspended in $2 \mathrm{~mL}$ PBS-1\% BSA. Viability was tested by staining the cells with trypan blue. The cell suspension was brought to a concentration of $2^{*} 10^{6}$ cells $/ \mathrm{mL}$ and $50 \mu \mathrm{L}$ of the suspension ( $10^{5}$ cells) was centrifuged to a pellet. Fifty microliters of serum was added to the pellet and incubated for 30 minutes at $21^{\circ} \mathrm{C}$. After two washes with $1 \mathrm{~mL}$ PBS-1\% BSA, $20 \mu \mathrm{L}$ of goat $\mathrm{F}\left(\mathrm{ab}^{\prime}\right) 2$ anti- 
human IgG FITC and $5 \mu \mathrm{L}$ PE- $\alpha \mathrm{CD} 3$ mouse monoclonal were added and incubated for 30 minutes at $4^{\circ} \mathrm{C}$ in the dark. The mixture was washed twice with cold PBS-1\% BSA, resuspended in $250 \mu \mathrm{L}$ PBS, and stored at $4^{\circ} \mathrm{C}$ in the dark until measurements were taken.

\section{Flow cytometry}

Cell samples were measured immediately after preparation in a FACScan flow cytometer (Becton Dickinson) using an argon laser (15 milliwatts) and the software program Lysis II (Becton Dickinson). Routine instrument function quality control included calibration and fluorescence compensation by test beads (Calibrite Beads, Becton \& Dickinson). Instrument settings were amplifier gain mode on a linear scale (256 channels) for FSC and SSC and on a logarithmic 4-log scale for FL1 (FITC) and FL2 (PE). The FSC threshold was set at 52 to eliminate acquisition of unwanted small particles. The fluorescence compensation FL1-FL2 by the test beads was usually $0.7 \%$.

In the FSC-SSC dotplot events with FSC 52-180 and SSC 0-200 were gated and this subset of lymphocytes was named R1. For each sample 10,000 events in gate R1 were acquired. The parameters FSC, SSC, FL1, and FL2 of each of these events were analyzed. Analysis was performed with the sofware programm Lysis II (Becton Dickinson). A dotplot was made of FSC versus FL2 of all events in gate R1 (Figure 4.4). The events were clustered into four groups and distinguished by FL2 $(>100$ or $<100)$ and FSC $(<90$ or $>90)$. Events with FL2 $<100$ were considered to be CD3-negative lymphocythes, while events with FL2 $>100$ were considered to be CD3-positive lymphocytes. The CD3-positive cluster with lower FSC (52-90) was gated R2. The CD3-positive cluster with higher FSC (90-180) was gated R3. Cells in both CD3-negative clusters had high background staining of FL1 and were considered to be non-T lymphocytes, most probably B lymphocytes. A histogram (FLl logarithmic versus number of events) was made from the events in gates R2 and R3 in the FSC-FL2 dotplot. The clearest discrimination between negative and positive control sera was seen in gate R3. Cells in gate R2 showed higher FLl fluorescence channels with the negative control sera, indicating more aspecific FITC staining compared to cells in gate R3. This might be caused by cells in gate R2 being less vital than cells in gate R3. Dead cells will nonspecifically absorb fluorescence-conjugated antiglobulins, resulting in higher background staining. R2 and R3 differ in the height of the FSC. The decrease in FSC when cells become less vital has previously been described. Chrest and coworkers studied the relationship between the FSC-SSC characteristics of murine T cells and their viability. Analysis of cells with low forward and high side scatter characteristics showed that this group contained (non)viable cells with apoptotic nuclei and necrotic cells $^{26}$. To avoid aspecific staining of these cells, we only analyzed the 
cells in gate R3 (FL2>100 and FSC >90) as being viable T lymphocytes for this thesis.

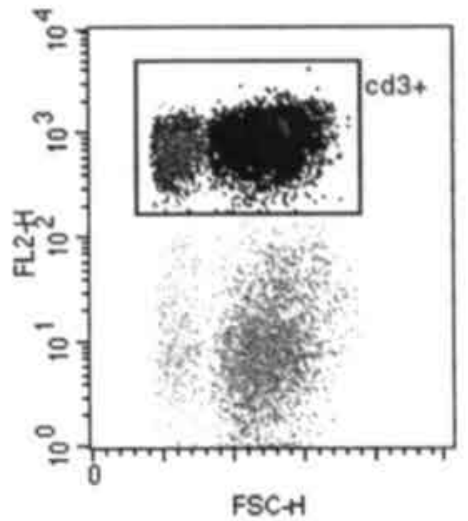

Figure 4.4 Dotplot of forward scatter vs. FL2 of lymphocytes gated on FSC 52-180, and SSC 0-200.

From the CD3 positive cells in gate R3 (events with FSC $90-180$ and SSC $0-200$ ) a histogram of FLl (FITC; logarithmic scale) was made. The median and mean channel were calculated using Lysis II software (Becton Dickinson). Comparison of positive and negative control sera, both neat and diluted, showed the median channel to discriminate best between positive and negative results. All FC crossmatch results in this thesis were calculated using the median channel.

To determine the optimal FITC concentration to be used, FITC-labelled antibody was tested at different dilutions against a positive control serum, CL8010 (Cedarlane, Ontario, Candada). CL8010 is a rabbit anti-human lymphocyte serum that is routinely used as positive control serum for HLA-ABC typing in standard microlymphocytotoxicity assays in our laboratory. Lymphocytes were incubated with CL8010 at dilutions of 1:16, 1:128, 1:256, 1:512, and 1:1024. A pool of negative human sera that had been extensively tested by both FC and $C D C$, was used as negative control. Goat F(ab')2 anti-human IgG FITC (Dakopatts, Glostrup, Denmark) was tested at dilutions of 1:8, 1:16, and 1:32. Further dilution of the goat $\mathrm{F}\left(\mathrm{ab}^{\prime}\right) 2$ anti-human IgG FITC resulted in lower FC results, as shown by previous studies. Results are presented in Figure 4.5 and Table 4.1. The FC result (median channel) was exponentially plotted on the $\mathrm{Y}$-axis as the ratio between test result and negative control serum, while the dilution of the serum is given on the $\mathrm{X}$-axis. For all $\mathrm{FC}$ crossmatches performed, goat $\mathrm{F}\left(\mathrm{ab}^{\prime}\right) 2$ anti-human IgG FITC was used in a dilution of 1:32 (Dakopatts, Glostrup Denmark). The positive control serum was MS106, which is an alloantiserum with $100 \%$ panel reactive antibodies 
that is routinely used for all our CDC tests. The negative control was pooled serum from 20 healthy, male blood bank donors.

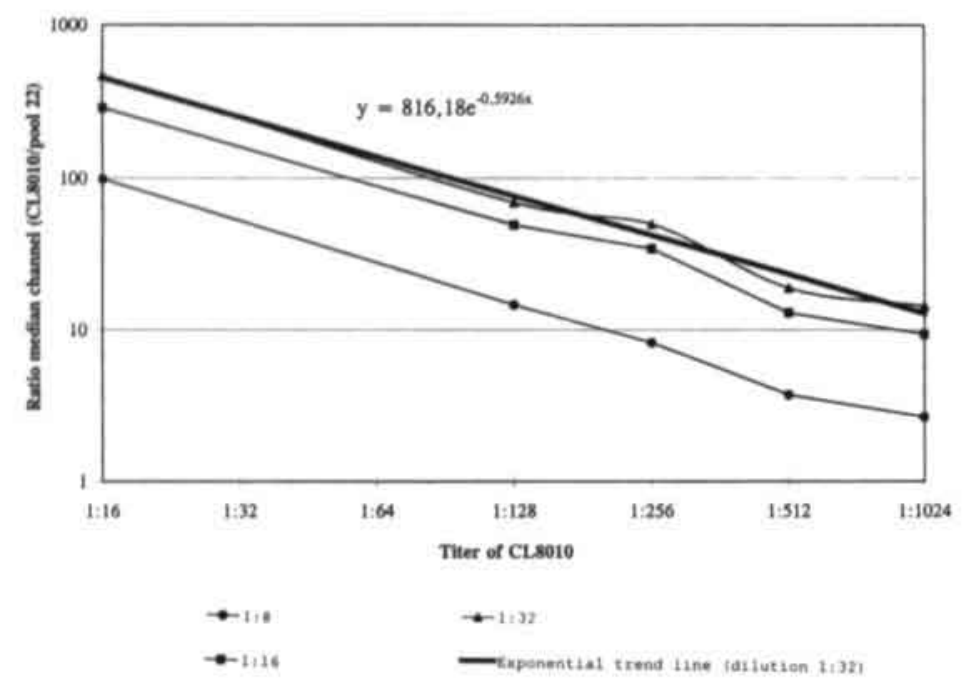

Figure 4.5 Titration curve FITC-IgG (batch 3202) for positive control serum (HLA-class I monoclonal antibody CL8010)

Table 4.1 Data of titration curve of CL8010 at different titers of FITC-IgG.

\begin{tabular}{llrrr}
\hline Serum & Dilution & & & \\
& & $\mathbf{1}: \mathbf{8}$ & $\mathbf{1}: \mathbf{1 6}$ & $\mathbf{1}: \mathbf{3 2}$ \\
\hline CL8010 & $1: 16$ & 98.96 & 286.77 & 464.46 \\
CL8010 & $1: 128$ & 14.70 & 49.19 & 69.00 \\
CL8010 & $1: 256$ & 8.27 & 34.33 & 49.91 \\
CL8010 & $1: 512$ & 3.75 & 13.00 & 18.89 \\
CL8010 & $1: 1024$ & 2.66 & 9.40 & 14.43 \\
Pool 21 & neat & 1.00 & 1.00 & 1.00 \\
\hline
\end{tabular}

\section{Definition of positive and negative $\mathrm{FC}$ crossmatches}

Positive and negative FC crossmatches were determined as described by Scornik et al. ${ }^{27}$. A panel of ten cells was tested against nine negative control sera. Crossmatch results were expressed as the fluorescence ratio between recipient serum and negative control (mean 1.185, SD 0.487; Table 4.2.). The cut-off point for positive and negative crossmatches was set at 2.65 (mean +3 SD). A crossmatch was regarded positive if the ratio of the median channel of the patient serum/negative control was $\geq 2.65$, provided the ratio positive control serum/negative control serum was $\geq 2.65$. The cut-off point for a negative crossmatch was set at 2.65 provided the ratio of the median channel of the positive control/negative control 
was $\geq 2.65$.

Table 4.2 Ratio of median channel of fluorescence of sera and pool 22.

\begin{tabular}{|c|c|c|c|c|c|c|c|c|c|c|c|}
\hline \multirow[b]{2}{*}{ cellno } & \multicolumn{3}{|c|}{ Positive sera } & \multirow[b]{2}{*}{ pook2 } & \multirow[b]{2}{*}{ m.c. } & \multirow[b]{2}{*}{92104} & \multirow[b]{2}{*}{92091} & \multirow[b]{2}{*}{92092} & \multirow[b]{2}{*}{92043} & \multicolumn{2}{|c|}{ Negative sena } \\
\hline & MS106 & MS106 & pooL1 & & & & & & & $A B 10$ & AB pool \\
\hline 111013 & 37.831 & 74.944 & 1.239 & 1.000 & 0.808 & 0.808 & 0.808 & 0.897 & 0.930 & 0.864 & 1.197 \\
\hline 122513 & 8.356 & 8.356 & 1.000 & 1.000 & 0.838 & 0.864 & 0.838 & 0.838 & 0.901 & 1.000 & 0.932 \\
\hline 122733 & 96.630 & nd & 1.000 & 1.000 & 1.000 & 1.000 & 1.036 & 1.196 & 1.783 & 1.080 & 1.333 \\
\hline 123673 & 103.859 & 148.495 & 1.288 & 1.000 & 0.935 & 1.076 & 1.076 & 0.967 & 1.158 & 1.245 & 1.337 \\
\hline 124283 & 74.987 & 50.481 & 1.487 & 1.000 & 1.000 & 1.117 & 1.240 & 1.117 & 1.071 & 1.331 & 1.597 \\
\hline 110773 & 184.435 & 96.513 & 1.382 & 1.000 & 1.157 & 0.901 & 1.157 & 0.963 & 0.838 & 3.277 & 1.115 \\
\hline 111653 & 179.346 & 25.463 & 0.748 & 1.000 & 0.866 & 0.833 & 0.776 & 0.776 & 0.724 & 1.114 & 0.898 \\
\hline 111763 & 293.961 & 236.886 & 1.074 & 1.000 & 1.153 & 1.332 & 1.908 & 1.114 & 1.332 & 1.432 & 1.432 \\
\hline 111953 & 183.785 & 468,342 & 1.430 & 1.000 & 1.483 & 1.772 & 4.054 & 1.430 & 1.591 & 1.235 & 1.906 \\
\hline 112193 & 197.904 & 92.966 & 0.837 & 1.000 & 1.000 & 0.803 & 1.242 & 0.966 & 1.000 & 1.152 & 1.382 \\
\hline
\end{tabular}

Mean natio of negative sera $=1.185 ;$ SD of ratio of negative sen $=0.487$

nd $=$ not done

Comparison of sensitivity of the FC crossmatch with sensitivity of the CDC crossmatch provides the relative sensitivity of the two techniques. Positivity in FC crossmatch was defined as a fluorescence ratio $\geq 2.65$. In Figure 4.5 the titration curve for positive control serum CL8010 with FITC-labelled anti IgG 1:32 is shown. The mathematical formula of the trend lines for this FITC dilution is $y=816.18 \mathrm{e}^{0.5926 x}$. According to this comparison, the ratio of 2.65 is reached at a dilution of CL8010 of $1: 13,000$. In the CDC crossmatch, the cut-off point for positivity ( $20 \%$ kill above background) is reached with the same serum at a dilution of 1:16. A similar increase in sensitivity was shown by Talbot and coworkers ${ }^{3}$.

Specificity of the FC crossmatch was studied by testing antisera with HLA class I specificity with positive and negative cells. Four polyclonal monospecific human allosera (anti-A2, -A1, -B57, and -B8, respectively) were studied with two different cells (HLA-types A2,A31;B7,B57 and A1,A3;B7,B8). Results are shown in Table 4.3. When the relevant HLA antigen was not present on the cells, the median channel of the FC crossmatch was the same as the median channel of the negative control serum. There were no false-positive results. When the relevant HLA antigen was present on the cells, the median channel of the FC crossmatch was positive. Correct dilutions had to be determined for every FITC batch. In this study, 1:16 was used. The FC crossmatch was also tested for sera containing anticlass II HLA alloantibodies (DR15 and DQ1). FC crossmatches with HLA class II positive sera did not differ from negative control sera; therefore, the FC crossmatch used was not influenced by the presence of class II antibodies. 
Table 4.3 Ratio of median channel of fluorescence of two different cells with antisera with class I specificity.

\begin{tabular}{|c|c|c|c|c|c|}
\hline Serum spec. & $\begin{array}{l}\text { cellno } \\
\text { HLA A and B } \\
\text { FITC }\end{array}$ & $\begin{array}{r}11101 \\
\text { A2A31;B7B57 } \\
1: 16 \\
\end{array}$ & $\begin{array}{r}11195 \\
\text { A1A3;B7B8 } \\
1: 16 \\
\end{array}$ & $\begin{array}{r}11101 \\
\text { A2A31;B7B57 } \\
1: 32 \\
\end{array}$ & $\begin{array}{r}11195 \\
\text { A1A } 3 ; \text { B7B8 } \\
1: 32 \\
\end{array}$ \\
\hline CL8010 & $1: 16$ & 115.517 & 121.817 & 77.819 & 99.942 \\
\hline CL8010 & $1: 64$ & 31.634 & 42.911 & 30.538 & 32.762 \\
\hline MS106 & $1: 2$ & 58.314 & 32.180 & 2.742 & 8.655 \\
\hline MS106 & $1: 16$ & 5.236 & 10.548 & 3.166 & 3.784 \\
\hline $\mathrm{A} 2$ & $1: 1$ & 7.776 & 0.981 & 9.658 & 0.963 \\
\hline $\mathrm{A} 1+\mathrm{A} 2$ & $1: 1$ & 6.497 & 4.610 & 3.929 & 1.979 \\
\hline B57 & $1: 1$ & 10.002 & 0.981 & 1.781 & 1.073 \\
\hline B8 & $1: 1$ & 1.037 & 10.935 & 1.000 & 4.866 \\
\hline pool 21 & neat & 1.114 & 1.175 & 1.038 & 1.037 \\
\hline pool 22 & neat & 1.000 & 1.000 & 1.000 & 1.000 \\
\hline $\mathrm{MC}$ & neat & nd & 0.882 & 1.056 & 0.930 \\
\hline
\end{tabular}

Positive result $=$ ratio $\geq 2.65$. Italic $=$ expected to be positive, bold $=$ positive result, $\mathrm{nd}=\mathrm{not}$ done

Positive control sera:

Negative control sera:

Antisera with specificity:
CL8010 (monoclonal) and MS106 (polyclonal). pool 21 and 22 (pooled sera from healthy male blood blank donors) and MC (serum from a healthy nontransfused male).

human allosera. 


\section{References}

1. Garovoy MR, Rheinschmidt MA, Bigos M, Perkins H, Colombe B, Feduska N, Salvatierra $\mathrm{O}$. Flow cytometry analysis: A high technology crossmatch technique facilitating transplantation. Transplant Proc 1983; 15: 1939-44.

2. Chapman JR, Deierhoi MH, Carter NP, Ting A, Morris PJ. Analysis of flow cytometry and cytotoxicity crossmatches in renal transplantation. Transplant Proc 1985; 17: 2480-1.

3. Talbot D, Givan AL, Shenton BK, Stratton A, Proud G, Taylor RMR. Rapid detection of low levels of donor specific IgG by flow cytometry with single and dual colour fluorescence in renal transplantation. J of Immunological Methods 1988; 112: $279-83$.

4. Leffell MS, Bray RA. Chapter V 1: Basic principles and quality assurance of immunofluorescence and flow cytometry. In: ASHI Laboratory Manual, 3rd edition. American Society for Histocompatibility and Immunogenetics. 1996.

5. Bray RA, Stempora L. Chapter V 2: Phenotyping by immunofluorescence. In: ASHI Laboratory Manual, 3rd edition. American Society for Histocompatibility and Immunogenetics. 1996.

6. Cook DJ, Scornik JC. Chapter V 3: Flow cytometry crossmatch. In: ASHI Laboratory Manual, 3rd edition. American Society for Histocompatibility and Immunogenetics. 1996.

7. Scornik JC. Chapter 20.3: Flow cytometry crossmatch. In: ASHI Laboratory Manual, 2nd edition. American Society for Histocompatibility and Immunogenetics. 1990.

8. Garovoy MR. Flow cytometry crossmatch testing in renal transplantation. Transplant Immunol Lett 1988; 5: 1-2.

9. Talbot D, Givan AL, Shenton BK, Proud G, Taylor RMR. The nature of donorspecific IgG isotypes identified by flow cytometry in the preoperative crossmatch. Transplant Proc 1988; 20 (1 suppl 1): 84-5.

10. Cook DJ, Terasaki PI, Iwaki Y, Terashita GY, Lau M. An approach to reducing early kidney transplant failure by flow cytometry crossmatching. Clin Transplant 1987; 1: 253-6.

11. Cook DJ, Terasaki PI, Iwaki Y, Terashita G, Fujikawa J, Gera J, Takeda A, Danovitch G, Rosenthal JT, Fine R, Ettenger R, Mendez R, Dainko E, Kavalich A, Martin D, Teichman S, Ward H, Koyle M, Berne T, Brennan LP, Lieberman E, Cohen A, Strauss F. Flow cytometry crossmatching for kidney transplantation. In: Terasaki PI, ed. Clinical Transplants 1988. UCLA Tissue Typing Laboratory L.A., 1988: $375-80$.

12. Kerman RH, Van Buren CT, Lewis RM, DeVera V, Baghdahsarian V, Gerolami K, Kahan BD. Improved graft survival for flow cytometry and antihuman-globulin crossmatch-negative retransplant recipients. Transplantation 1990; 49: 52-6.

13. Talbot D, Givan AL, Shenton BK, Stratton A, Proud G, Taylor RMR. The relevance of a more sensitive crossmatch assay to renal transplantation. Transplantation 1989; 47: 552-5. 
14. Thistlethwaite JR, Buckingham M, Stuart JK, Gaber AO, Mayes JT, Stuart FP. T cell immunofluorescence flow cytometry cross-match results in cadaver donor renal transplantation. Transplant Proc 1987; 19: 722-4.

15. Talbot D, Cavanagh G, Coates E, Givan AL, Shenton BK, Lennard TWJ, Proud G, Taylor RMR. Improved graft outcome and reduced complications due to flow cytometric crossmatching and DR matching in renal transplantation. Transplantation 1992; 53: 925-8.

16. Kerman RH, Kimball PM, van Buren CT, Lewis RM, DeVera V, Baghdahsarian V, Heydari A, Kahan BD. AHG and DTE/AHG procedure identification of crossmatch-appropriate donor-recipient pairings that result in improved graft survival. Transplantation 1991; 51: 316-20.

17. Scornik JC, Brunson ME, Schaub B, Howard RJ, Pfaff WW. The crossmatch in renal transplantation: evaluation of flow cytometry as a replacement for standard cytotoxicity. Transplantation 1994; 57: 621-5.

18. Ogura K, Terasaki PI, Johnson C, Mendez R, Rosenthal JT, Ettenger R, Martin DC, Dainko E, Cohen L, Mackett T, Berne T, Barba L, Lieberman E. The significance of a positive flow cytometry crossmatch test in primary kidney transplantation. Transplantation 1993; 56: 294-8.

19. Iwaki Y, Cook DJ, Terasaki PI, Lau M, Terashita GY, Danovitch G, Fine R, Ettenger R, Mendez P, Kavalich A, Martin D, Soderblom R, Ward H, Berne T, Lieberman E, Straus F. Flow cytometry crossmatching in human cadaver kidney transplantation. Transplantation Proc 1987; 19; 1; 764-6.

20. Lazda VA, Pollak R, Mozes MF, Jonasson O. The relationship between flow cytometer crossmatch results and subsequent rejection episodes in cadaver renal allograft recipients. Transplantation 1989; 45: 562-5.

21. Talbot D, Givan AL, Shenton BK, Stratton A, Coates E, Parrott MR, Forsythe JLR, Rigg K, Lennard TWJ, Proud G, Taylor RMR. The prospective value of the preoperative flow cytometric crossmatch assay in renal transplantation. Transplantation 1990; 49: 809-10.

22. Karuppan SS, Ohlman S, Möller E. The occurrence of cytotoxic and noncomplement-fixing antibodies in the crossmatch serum of patients with early acute rejection episodes. Transplantation $1992 ; 54 ; 839-43$.

23. Karuppan SS, Lindholm A, Möller E. Characterization and significance of donorreactive B cell antibodies in current sera of kidney transplant patients. Transpantation 1990; 49: 510-5.

24. Karuppan SS, Lindholm A, Möller E. Fewer acute rejection episodes and improved outcome in kidney-transplanted patients with selection criteria based on crossmatching. Transplantation 1992; 53: 666-73.

25. van den Berg-Loonen E, Overhof R. Tillemans $M$, van Hooff H. Allo and auto crossmatches after transplantation. Transpl Int. 1992, vol 5 (suppl 1); S 594-5.

26. Chrest FJ, Buchholz MA, Kim YH, Kwon TK, Nordin AA. Identification and quantification of apoptotic cells following anti-CD3 activation of murine $\mathrm{G}_{0} \mathrm{~T}$ cells. Cytometry 1993; 14: 883-90. 
27. Scornik JC, Salomon DR, Lim PB, Howard RJ, Pfaff WW. Posttransplant antidonor antibodies and graft rejection: evaluation by two-color flow cytometry. Transplantation 1989; 47: 287-90. 


\section{Chapter 5}

No advantage of flow cytometry crossmatch over complementdependent cytotoxicity in immunologically welldocumented renal allograft recipients

MHL Christiaans, R Overhof, A ten Haaft, F Nieman, JP van Hooff, EM van den Berg-Loonen

Transplantation 1996; 62: 1341-7 


\begin{abstract}
The effect of flow cytometry crossmatches on clinical outcome was studied retrospectively in two groups of immunologically well-documented patients who had received transplants with a negative complement-dependent cytotoxicity crossmatch. The first group consisted of 114 consecutive renal allograft recipients, and the second group consisted of 76 immunologically at-risk recipients. Flow cytometry crossmatches were performed with current and historic sera.
\end{abstract}

In group 1, positive flow cytometry (FC) crossmatches were shown in 15/114 (13\%) recipients. Rejection occurred in 8/15 (53\%) FC-positive versus 41/99 (41\%) FC-negative recipients. The 1-year graft survival rate was $80 \%$ for FC-positive patients and $87 \%$ for FC-negative patients. Sixty-seven patients were nonsensitized patients; 4 of them had a positive FC crossmatch but no rejection episodes, graft loss, or patient loss. Of 47 retransplanted and/or sensitized recipients 11 had a positive FC crossmatch. Rejection treatment was needed in 8/11 (73\%) FC-positive patients compared with 19/36 (53\%) FCnegative patients. Their 1-year graft survival rates were $73 \%$ and $81 \%$. None of these differences reached statistical significance.

Group 2 consisted of 76 at-risk recipients; 37 were retransplant patients and 39 were sensitized first transplant patients. Twenty-one (28\%) patients showed a positive FC crossmatch. Rejection episodes did not differ between the FC-positive $(48 \%)$ and FCnegative patients $(46 \%)$. There was no difference in 1-year graft survival rate $(76 \%$ vs. $80 \%)$ or in 1-year patient survival rate (100\% vs. $95 \%)$.

We conclude that FC crossmatches in our patient group are not superior to the classical complement-dependent cytotoxicity crossmatches with regard to clinical outcome. On the contrary, transplantation with a mandatory negative FC crossmatch would have excluded $28 \%$ of the recipients from transplantation, who in fact are doing well. 


\section{Introduction}

Pretransplant crossmatching for renal transplantation has been routinely performed since the late 1960s. The complement-dependent cytotoxicity (CDC) crossmatch is the most commonly used histocompatibility technique, historically referred to as the National Institutes of Health $(\mathrm{NIH})$ crossmatch. To accomplish an increase in sensitivity, other crossmatch techniques have been introduced. Variants of the NIH technique have been added by modifying the incubation time or temperature, the number of wash steps, and the addition of antiglobulin reagent. New crossmatch techniques have also been used, one of these being the flow cytometry (FC) crossmatch. The FC crossmatch technique is used predominantly in the United States, but an increasing number of European laboratories are in favor of this technique. The main advantages mentioned are increased sensitivity ${ }^{1,2}$, detection of non-complement-fixing antibodies ${ }^{3.4}$, and correlation with transplant outcome ${ }^{5.9}$. The main disadvantages are the large number of cells needed to perform a test, the amount of serum required, and the initial cost of the apparatus.

Patients who had received transplants with a positive FC crossmatch and a negative NIH crossmatch were shown to have decreased graft survival. This was demonstrated for first transplants ${ }^{5,10}$ as well as retransplants ${ }^{6,7}$. Also, the number of rejection eposodes were correlated with positive FC crossmatch $^{7.9}$. There was not much difference in clinical outcome when the FC crossmatch results were compared with the anti-human immunoglobulin test? ${ }^{7}$. In studies comparing the effect of FC crossmatching with that of CDC crossmatching, different CDC crossmatches were used $^{7,11-13}$. Current sera were used ${ }^{6}$ as well as historical sera ${ }^{11-13}$. In some studies, autoantibodies ${ }^{12.13}$ or class II antibodies ${ }^{13}$ were tested for.

There are different protocols for performing FC crossmatches. They differ in the type of antibody used for measuring cell-bound antibody $y^{2,6,8}$, the expression of the channel fluorescence ${ }^{2.6,8}$, and their definition of positivity ${ }^{2.6,11-13}$. The label used for cell-bound antibody was specific anti-immunoglobulin $\mathrm{G}^{6.8}$ or total antiimmunoglobulin ${ }^{2}$. Fluorescence was expressed as mean ${ }^{2.8}$ or median peak channel ${ }^{6}$. Positivity was expressed as the number of channel shifts ${ }^{2,6,11,12}$ or as the fluorescence ratio of test and control sera ${ }^{13}$. The FC crossmatch was performed for $\mathrm{T}$ cells $^{10}$ or for $\mathrm{T}$ and $\mathrm{B}$ cells $\mathrm{s}^{6,8,13}$. The serum used for the FC crossmatch was current serum ${ }^{6,8}$ or included peak serum as well ${ }^{12,13}$.

In comparing $\mathrm{FC}$ and $\mathrm{CDC}$ crossmatches, the role of other factors that influence transplant outcome should be taken into account. Besides screening and crossmatch techniques, the local policy of selecting donor-recipient-combinations and the posttransplant immunosuppressive regimen ${ }^{7,14-16}$ greatly influence transplant outcome. It is possible, therefore, that the claimed advantages of FC crossmatch over CDC crossmatch also depend on the local regimen concerning tissue typing. Transplantation in our center is performed under the auspices of Eurotransplant. 
Matching priority is HLA-DR, HLA-B, and HLA-A, respectively. Selection criteria used for transplantation include a mandatory negative class I CDC crossmatch at the time of transplantation with current as well as historic sera. HLA antigens are considered not acceptable for a given patient if the particular specificity was demonstrable in any previous screening test. In female patients, the paternally inherited HLA antigens of the children were excluded from the transplants.

The outcome of patients with a positive FC crossmatch and a negative CDC crossmatch identifies the clinical value of FC crossmatching. Therefore, we decided to determine the result of $\mathrm{FC}$ crossmatching in a consecutive group of patients (group 1) who had received transplants in our center over a 4-year period. All patients received transplants according to the above-mentioned criteria and were treated with cyclosporine (CsA) as a basic immunosuppressive drug. Subsequently, a second patient group (group 2) was studied to investigate the effect of FC crossmatch in patients who were considered to be immunologically at risk. All patients in this group were either sensitized or retransplanted due to immunological failure of a previous graft within 1 year after transplantation.

\section{Materials and Methods}

\section{Patients}

Two different patient groups were studied. All were treated with CsA. Group 1 consisted of 140 consecutive cadaveric kidney graft recipients who had received transplants from January 1986 up to and including December 1989 in our center. All recipients had negative $\mathrm{NIH}$ crossmatches at the time of transplantation. They were tested retrospectively by $\mathrm{FC}$ crossmatch against donor $\mathrm{T}$ cells and autologous $\mathrm{T}$ cells. In 114 combinations, donor and recipient lymphocytes were available. Their characteristics are presented in Table 5.1. Group 2 consisted of patients who were sensitized (panel reactive antibody (PRA) $>5$, i.e., immunized or highly immunized according to Eurotransplant criteria) or who were retransplanted due to immunological failure of a previous graft within 1 year after transplantation. From January 1983 up to and including December 1992, 100 patients, who fulfilled these criteria, were transplated in our center. Lymphocytes were available from 76 donor-recipient combinations. The characteristics of group 2 are presented in Table 5.1. Of 76 patients, 47 were also included in the first group. 
Table 5.1 Pretransplant recipient and donor characteristics*

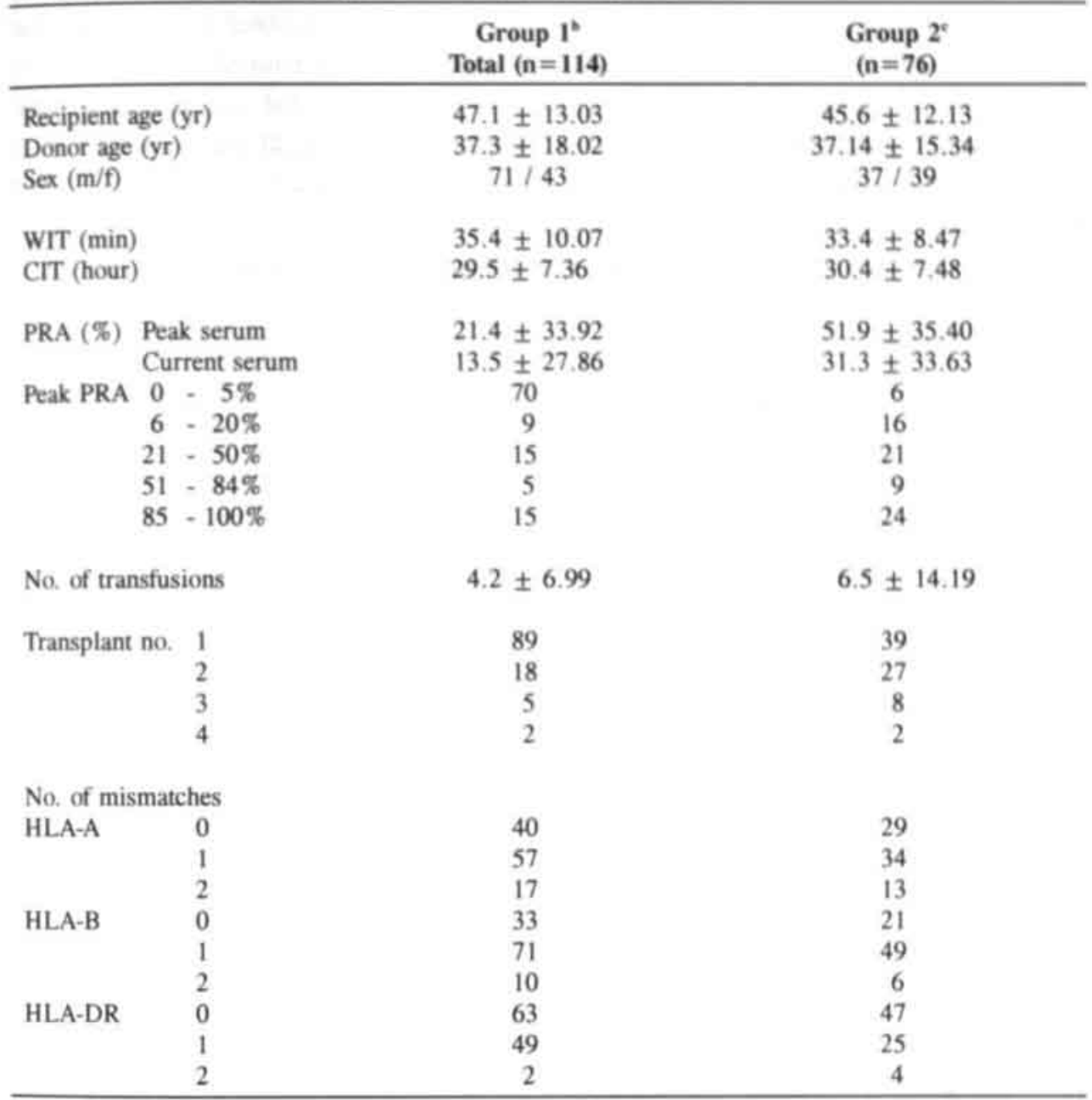

* Data are given as numbers or mean $\pm \mathrm{SD}$

- Consecutive transplants from 1986 through 1989

"All patients at risk (1983-1992); sensitized or regrafted due to immunological failure of a previous graft within 1 year after transplant.

\section{Immunosuppression}

The immunosuppresive regimen consisted of CsA and low-dose prednisolone (PRED; $10 \mathrm{mg} /$ day) for recipients of first grafts. The CsA was started intravenously before surgery (continuous infusion: $4 \mathrm{mg} / \mathrm{kg}$ every $24 \mathrm{hr}$ ), and switched orally $(4 \mathrm{mg} / \mathrm{kg}$ b.i.d.) at day 2 . The PRED dose was diminished to $7.5 \mathrm{mg} /$ day at month 1 and to $5 \mathrm{mg} /$ day at month 3 . For recipients without rejection, the PRED dose was further tapered to $0 \mathrm{mg}$ in the next months. For 
highly immunized recipients (PRA $>85 \%$ ) and retransplant recipients, azathioprine (AZA, $\pm 1 \mathrm{mg} / \mathrm{kg}$ body weight) was added to the above-described regimen and the dose of PRED was tapered to $5 \mathrm{mg}$. The CsA level was determined in whole blood by high-performance liquid chromatography or by monoclonal radioimmunoassay. The trough CsA level for CsA+PRED recipients was $0.15-0.20 \mathrm{mg} / \mathrm{L}$ in the first 3 months after transplantation and $0.10-0.15 \mathrm{mg} / \mathrm{L}$ after 3 months. For CsA + PRED + AZA recipients, the trough CsA level was $0.10-0.15 \mathrm{mg} / \mathrm{L}$ in the first 3 months after transplantation and $0.05-0.10 \mathrm{mg} / \mathrm{L}$ after 3 months.

\section{Clinical outcome parameters}

Rejection is defined as any rejection treatment in the first 6 months after grafting. In general, rejection episodes were proven by needle core biopsy. Rejection treatment consisted of a 10-day course of rabbit antilymphocyte globulin (RIVM, Bilthoven, the Netherlands). For patients who had received rabbit antilymphocyte globulin in the past or who had a seriously compromised cardiovascular system, the rejection was treated with three doses of methylprednisolone (0.5-1.0 g/dose).

Graft failure was defined as loss of kidney function but also included death of recipient with functioning graft. No exclusions were made.

\section{Center policy of tissue typing}

Nontransfused patients received at least one leukocyte-poor blood transfusion before transplantation. Serum was collected at weekly intervals, i.e., weeks 0,2 , 3, and 4. Each sample was investigated for the development of HLA antibodies using crossmatches with blood donor lymphocytes.

Serum samples were collected every 3 months during the time patients were on the waiting list. If after the initial transfusion additional blood transfusions were required, only filtrated, leukocyte-depleted blood was used. Serum was also collected after these transfusions. Screening for the presence of anti-HLA class I and II antibodies was performed with and without dithiothreitol (DTT) by using a select panel of 64 lymphocytes. Only DTT-resistant T-cell reactive antibodies were included in the PRA. Reading was performed using a Leitz Patimed automated microfluorometer microscope and positivity was defined as at least $40 \%$ cell death by the patient serum compared with three positive control sera (100\%). Routinely, all serum samples obtained from each patient were investigated every 3 months. All samples were investigated for the presence of autoantibodies by crossmatching with autologous patient lymphocytes.

HLA antigens were considered not acceptable for a patient if antibodies against the specificity had been demonstrated either at the time of transplantation or in the past. Also, mismatches from previous transplants were excluded, as were the paternally inherited antigens of the children in female patients. Transplants were 
performed under the auspices of Eurotransplant. Matching priority was HLA-DR, HLA-B, and HLA-A.

\section{Crossmatches}

Conventional crossmatches in our center included three complement-dependent crossmatches: the standard NIH crossmatch, the two-color fluorescence crossmatch, and a DTT crossmatch in which the serum was treated with DTT to reduce $\operatorname{lgM}$ antibodies. Sera used for crossmatches at the time of transplant were the current serum drawn at the time of transplantation, the last screened serum sample, and all necessary positive historical samples (peak sera). A negative class 1 crossmatch $(\mathrm{NIH})$ was mandatory for transplantation. This holds for current as well as for historical sera. Routinely, class I (NIH), class II (two-color fluorescence), and DTT crossmatches were performed for current and historically positive sera. All crossmatches as well as screening results were used in the interpretation of the crossmatch results. That is, if a class I crossmatch turned negative after DTT treatment, the crossmatch was interpreted as negative, provided no HLA-specific antibodies had been demonstrated by screening. For the present study, all serum samples tested at the time of transplantaton were retested by CDC and FC crossmatching using liquid-nitrogen-stored spleen lymphocytes of the donor. For antibody-negative patients with no historically positive serum, the serum drawn 4 weeks after the leukocyte-poor blood transfusion was used as the "peak" serum. For antibody-negative retransplant patients, a serum sample taken after failure of the graft, preferably after transplantectomy, was used as peak serum. All sera were also investigated for the presence of autoantibodies using crossmatches with recipient peripheral blood lymphocytes stored in liquid nitrogen.

\section{Flow Cytometry}

The FC crossmatch technique was performed as described by Scornik et al. ${ }^{13}$ Frozen lymphocytes were thawed in $8 \mathrm{~mL}$ of RPMI containing $20 \%$ fetal calf serum and washed twice in $8 \mathrm{~mL}$ of phosphate-buffered saline (PBS) with $1 \%$ bovine serum albumin (BSA). Cells were centrifuged for $5 \mathrm{~min}$ at $700 \mathrm{xg}$. Lymphocytes $\left(10^{5}\right)$ were centrifuged to a pellet, the supernatant was removed, and cells were tested for viability. The cells were incubated with $50 \mu \mathrm{L}$ of serum for 30 min at $21^{\circ} \mathrm{C}$. After washing twice with $8 \mathrm{~mL}$ of PBS-BSA $1 \%, 20 \mu \mathrm{L}$ of a fluorescein-conjugated $\mathrm{F}(\mathrm{ab})$ '2 goat-anti-human IgG (Tago, Burlingame, CA) diluted 1:16 were added, as well as $5 \mu \mathrm{L}$ of phycoerythrin-conjugated anti-CD3 mouse monoclonal antibody (Dakopatts, Glostrup, Denmark), and incubated for 30 minutes at $4^{\circ} \mathrm{C}$. Finally, cells were washed twice with $8 \mathrm{~mL}$ of PBS-BSA $1 \%$ and resuspended in $250 \mu \mathrm{L}$ of PBS. For all donor crossmatches, spleen lymphocytes were incubated with (1) the recipient's current serum, (2) the recipient's peak PRA 
serum, (3) a positive control serum, and (4) a negative control serum. The positive control was an alloantiserum routinely used in our laboratory with $100 \%$ PRA. The negative control was pooled serum from 20 healthy male blood bank donors. Autocrossmatches were performed using recipient peripheral blood lymphocytes and were tested against the same sera used in the donor crossmatches.

FC crossmatching was performed with a FACScan flow cytometer (Becton Dickinson, 256 channels, logarithmic scale). Routine instrument function quality control included calibration by test beads and fluorescence compensation (Calibrite, Becton Dickinson). From each sample 10,000 cells were analysed in the lymphocyte gate. From the $\mathrm{CD}^{+}$cells, a histogram of fluorescein isothiocyanate (logarithmic scale) was made. The median and mean channel were calculated. Comparison of positive (neat + diluted) and negative control sera showed the median channel to give the best discrimination between positive and negative results. All FC crossmatch results in this analysis were calculated from the median channel.

Positive and negative FC crossmatch was determined as described by Scornik et al. ${ }^{17}$ : a panel of 10 cells was tested against 9 negative control sera. Crossmatch results were expressed as the fluorescent ratio between recipient serum and negative control (mean 1.185, SD 0.487). The cutoff point for positive and negative crossmatches was set at 2.65 (mean $+3 \mathrm{SD}$ ). A crossmatch was regarded as positive if the ratio of the median channel of the patient serum to the negative control was $\geq 2.65$, provided that the ratio of positive control serum to negative control was $\geq 2.65$.

The cutoff point for a negative crossmatch was set at 2.65 provided the ratio of the median channel of the positive control to the negative control was $\geq 2.65$.

Statistical analysis was performed using SPSS/PC+ version 4.0. Pearson chisquare test, odds ratio analysis, Mann-Whitney $U$ test and independent groups Student's $t$ test were performed when indicated. A $P$-value $<0.05$ was considered to be statistically significant.

\section{Results}

\section{Group 1: Consecutive Patients}

FC crossmatch results

Of 114 recipients, 15 had a positive FC crossmatch (13\%). Eleven showed a current positive FC crossmatch $(9.6 \%)$, while 10 had a peak positive FC crossmatch $(8.8 \%)$. Six recipients were found te be FC crossmatch positive with peak as well as current serum. Forty-seven patients were sensitized or received regrafts (at risk), whereas 67 patients were nonsensitized. From the nonsensitized 
group, four first-graft recipients were positive, two with current serum and two with peak serum (Table 5.2).

FC crossmatch-positive recipients had statistically significant higher peak and current PRA compared with FC crossmatch-negative recipients (Mann-Whitney $U$ test $P<0.01$ ). No significant differences were shown for the other pretranspant characteristics (Table 5.3).

Table 5.2 FC crossmatches and clinical outcome in 114 consecutive cadaveric renal allografts (group 1$)^{\circ}$

\begin{tabular}{|c|c|c|c|c|c|c|}
\hline \multirow[t]{2}{*}{ FC crossmatch } & \multicolumn{2}{|c|}{ Rejection } & \multicolumn{2}{|c|}{$\begin{array}{l}\text { 1-year graf } \\
\text { survival }\end{array}$} & \multicolumn{2}{|c|}{$\begin{array}{l}\text { 1-year patient } \\
\text { survival }\end{array}$} \\
\hline & $\mathbf{n}$ & (\%) & $\mathbf{n}$ & (\%) & $\mathbf{n}$ & (\%) \\
\hline \multicolumn{7}{|l|}{ Current serum } \\
\hline \multicolumn{7}{|l|}{ Non-sensitized graft } \\
\hline FC positive $(n=2)$ & 0 & (0) & 2 & (100) & 2 & $(100)$ \\
\hline FC negative $(n=65)$ & 22 & (34) & 59 & (91) & 62 & (95) \\
\hline \multicolumn{7}{|l|}{ Sensitized/regrafts } \\
\hline FC positive $(n=9)$ & 6 & (67) & 7 & (78) & 9 & $(100)$ \\
\hline FC negative $(n=38)$ & 21 & (55) & 30 & (79) & 36 & (95) \\
\hline \multicolumn{7}{|l|}{ All recipients } \\
\hline FC positive $(n=11)$ & 6 & (55) & 9 & (82) & 11 & $(100)$ \\
\hline FC negative $(n=103)$ & 43 & (42) & 89 & (86) & 98 & (95) \\
\hline \multicolumn{7}{|l|}{ Peak serum } \\
\hline \multicolumn{7}{|l|}{ Non-sensitized graft } \\
\hline FC positive $(\mathrm{n}=2)$ & 0 & (0) & 2 & $(100)$ & 2 & $(100)$ \\
\hline FC negative $(n=65)$ & 22 & (34) & 59 & (91) & 62 & (95) \\
\hline \multicolumn{7}{|l|}{ Sensitized/regrafts } \\
\hline FC positive $(n=8)$ & 5 & (63) & 5 & (63) & 8 & $(100)$ \\
\hline$F C$ negative $(n=39)$ & 22 & (56) & 32 & (82) & 37 & (95) \\
\hline \multicolumn{7}{|l|}{ All recipients } \\
\hline FC positive $(\mathrm{n}=10)$ & 5 & (50) & 7 & (70) & 10 & $(100)$ \\
\hline FC negative $(n=104)$ & 44 & (42) & 91 & (88) & 99 & (95) \\
\hline \multicolumn{7}{|l|}{ Peak or current serum } \\
\hline \multicolumn{7}{|l|}{ Non-sensitized graft } \\
\hline FC positive $(\mathrm{n}=4)$ & 0 & (0) & 4 & (100) & 4 & $(100)$ \\
\hline FC negative $(n=63)$ & 22 & (35) & 57 & (91) & 60 & (95) \\
\hline \multicolumn{7}{|l|}{ Sensitized/regrafts } \\
\hline FC positive $(n=11)$ & 8 & (73) & 8 & (73) & 11 & $(100)$ \\
\hline FC negative $(n=36)$ & 19 & (53) & 29 & (81) & 34 & (94) \\
\hline \multicolumn{7}{|l|}{ All recipients } \\
\hline FC positive $(n=15)$ & 8 & (53) & 12 & (80) & 15 & $(100)$ \\
\hline FC negative $(\mathrm{n}=99)$ & 41 & (41) & 86 & (87) & 94 & (95) \\
\hline
\end{tabular}

- None of the comparisons between FC crossmatch result and clinical outcome reached statistical significance by chi-square test nor by odds ratio analysis.

\section{Clinical outcome}

The 1-year patient survival rate was $100 \%$ for recipients of positive FC crossmatch grafts (15/15) compared with $95 \%$ for recipients of negative FC crossmatch grafts (94/99) (Table 5.2). This was true for peak as well as current sera.

The 1-year graft survival rate was $82 \%$ for current positive FC crossmatch recipients (9/11) and $86 \%$ for current negative FC crossmatch recipients $(89 / 103)$. For peak positive and negative FC crossmatch recipients, this was $70 \%(7 / 10)$ and 
$88 \%(91 / 104)$. When either peak or current FC crossmatch was positive, the 1-year graft survival rate was $80 \%(12 / 15)$; when negative, the rate was $87 \%(86 / 99)$ (Table 5.2).

Table 5.3 Pretransplant recipient and donor characteristics in 114 consecutive cadaveric renal allografts (group i) based on FC crossmatch result".

\begin{tabular}{|c|c|c|c|}
\hline & & $\begin{array}{c}\text { FCXM positive } \\
(n=15)\end{array}$ & $\begin{array}{c}\text { FCXM negative } \\
(\mathrm{n}=99)\end{array}$ \\
\hline \multicolumn{2}{|c|}{ Recipient age (yr) } & $48.3 \pm 13.93$ & $46.9 \pm 12.96$ \\
\hline \multicolumn{2}{|l|}{ Donor age (yr) } & $34.2 \pm 15.45$ & $37.8 \pm 18.41$ \\
\hline \multicolumn{2}{|l|}{$\operatorname{Sex}(m / f)$} & $9 \longdiv { 6 }$ & $62 / 37$ \\
\hline \multicolumn{2}{|l|}{ WIT (min) } & $35.3 \pm 10.49$ & $35.5 \pm 10.06$ \\
\hline \multicolumn{2}{|l|}{ CIT (hour) } & $29.1 \pm 7.14$ & $29.6 \pm 7.43$ \\
\hline \multicolumn{2}{|c|}{ PRA (\%) Peak serum } & $45.7 \pm 42.00$ & $17.7 \pm 31.15^{b}$ \\
\hline \multicolumn{2}{|c|}{ Current serum } & $35.3 \pm 37.34$ & $10.2 \pm 24.73^{b}$ \\
\hline \multirow{5}{*}{$\begin{array}{r}\text { Peak PRA } \\
2 \\
5 \\
8 \\
5\end{array}$} & $-5 \%$ & 5 & 65 \\
\hline & $-20 \%$ & 2 & 7 \\
\hline & $-50 \%$ & 1 & 14 \\
\hline & $-84 \%$ & 2 & 3 \\
\hline & $-100 \%$ & 5 & 10 \\
\hline \multicolumn{2}{|c|}{ Number of transfusions } & $7.5 \pm 9.21$ & $3.7 \pm 6.56$ \\
\hline \multirow[t]{4}{*}{ Transplant no. } & 1 & 9 & 80 \\
\hline & 2 & 5 & 13 \\
\hline & 3 & 1 & 4 \\
\hline & 4 & 0 & 2 \\
\hline \multicolumn{4}{|c|}{ Number of mismatches } \\
\hline \multirow[t]{3}{*}{ HLA-A } & 0 & 4 & 36 \\
\hline & 1 & 8 & 49 \\
\hline & 2 & 3 & 14 \\
\hline \multirow[t]{3}{*}{ HLA-B } & 0 & 7 & 26 \\
\hline & 1 & 7 & 64 \\
\hline & 2 & 1 & 9 \\
\hline \multirow[t]{3}{*}{ HLA-DR } & 0 & 12 & 51 \\
\hline & 1 & 3 & 46 \\
\hline & 2 & 0 & 2 \\
\hline
\end{tabular}

- Data are given as numbers or mean $\pm S D$. Group 1 comprised consecutive transplant patients from 1986 through 1989. No statistical significance was shown by Mann-Whitney $U$ test, unpaired Student's $t$ test, or chi-square test between FC crossmatch results and the other characteristics of donor or recipient

" $P<0.01$ by Mann-Whitney $U$-test. 
Rejection treatment was needed for 6 of 11 (55\%) recipients with a current positive FC crossmatch in the first 6 months after transplantation, compared with 43 of 103 (42\%) of those with a current negative FC crossmatch (Table 5.2). For peak positive and negative FC crossmatch recipients, the results were $50 \%$ and $42 \%$, respectively. Of 15 peak and/or current FC crossmatch-positive recipients, 8 needed rejection treatment (53\%) compared with 41 of 99 FC crossmatch-negative recipients. None of the above-mentioned differences reached statistical significance (Table 5.2).

In sensitized or retransplanted recipients, more rejection treatments were needed in patients with positive and negative FC crossmatches. The number of rejections was slightly higher in the positive FC crossmatch patients (73\%), and the 1-year graft survival rate was slightly lower $(73 \%)$. None of these differences was statistically significant. This lack of significance might be the result of the relatively small number of patients in this group (47). Therefore, an additional 29 recipients at risk, i.e., sensitized and/or retransplanted, were selected and added to the initial group. thus constituting group 2 .

\section{Group 2: patients at risk}

\section{$F C$ crossmatch results}

Twenty-one of 76 recipients at risk ( $28 \%)$ had a positive FC crossmatch with either current or peak serum. Fourteen (18\%) showed a current positive FC crossmatch, while $17(22 \%)$ had a peak positive FC crossmatch (Table 5.4). Ten recipients were positive with both current and peak serum. There were no significant differences in pretransplant characteristics between FC crossmatch-positive and FC crossmatch-negative recipients (Table 5.5).

\section{Clinical outcome}

The 1-year patient survival rate was $100 \%$ for current positive FC crossmatch patients (14/14) and 95\% for current negative FC crossmatch patients (59/62) (Table 5.4). For peak positive FC crossmatch, the same survival rate was found.

The 1-year graft survival rate was $86 \%$ for current positive FC crossmatch (12/14) and $77 \%(48 / 62)$ for current negative FC crossmatch. When patients with peak positive FC crossmatches were taken into account, the 1-year graft survival rate was $76 \%(16 / 21)$.

Rejection treatment was needed in 7 of 14 recipients with a current positive FC crossmatch (50\%) compared with 28 of 62 with a current negative FC crossmatch (45\%) (Table 5.4). These data did not alter significantly when peak positive crossmatches were taken into account ( $48 \%$ vs. $46 \%)$. No significant difference in clinical parameters could be demonstrated between positive and negative FC crossmatch patients. FC crossmatch positive and negative recipients had the same clinical course in the first year after transplantation. 
Table 5.4 Flow cytometry crossmatches and clinical outcome in 76 cadaveric renal allografts immunologically at risk (group 2$)^{2}$

\begin{tabular}{|c|c|c|c|c|c|c|}
\hline FC crossmatch & \multicolumn{2}{|c|}{$\begin{array}{l}\text { Rejection } \\
\mathbf{n} \quad(\%)\end{array}$} & \multicolumn{2}{|c|}{$\begin{array}{c}\text { 1-year graft survival } \\
\text { n }(\%)\end{array}$} & \multicolumn{2}{|c|}{$\begin{array}{r}\text { 1-year patient survival } \\
\mathbf{n} \quad(\%)\end{array}$} \\
\hline \multicolumn{7}{|l|}{ Current serum } \\
\hline FC $\operatorname{pos}(n=14)$ & 7 & $(50)$ & 12 & (86) & 14 & (100) \\
\hline FC neg $(n=62)$ & 28 & (45) & 48 & (77) & 59 & (95) \\
\hline \multicolumn{7}{|l|}{ Peak serum } \\
\hline FC $p o s(n=17)$ & 6 & (35) & 12 & (71) & 17 & $(100)$ \\
\hline FC neg $(\mathrm{n}=59)$ & 29 & (49) & 48 & (81) & 56 & (95) \\
\hline \multicolumn{7}{|c|}{ Peak or current serum } \\
\hline FC $p o s(n=21)$ & 10 & (48) & 16 & (76) & 21 & $(100)$ \\
\hline FC neg $(n=55)$ & 25 & (46) & 44 & (80) & 52 & (95) \\
\hline
\end{tabular}

" Group 2 comprised all patients at risk (1983-1992), sensitized or regrafted due to immunological failure of a previous graft within I year after transplant. None of the comparisons between FC crossmatch result and clinical outcome reached statistical significance by chi-square test nor by odds ratio analysis.

\section{Discussion}

Irreversible rejection and subsequent graft loss due to antibody-mediated reactivity is one of the important problems in renal transplantation. The incidence of primary nonfunctioning grafts is higher in sensitized and regrafted recipients ${ }^{18}$. Regrafts have a lower graft survival rate if patients have rejected their first graft between 1 month and 1 year after transplantation ${ }^{19}$. Presensitization of recipients may not always be detected by conventional crossmatch tests based on CDC. FC crossmatches are supposed to be an additional and highly reliable tool in the detection of antidonor antibodies in pretransplant recipient sera.

The outcome of transplantation is determined by many factors besides the crossmatch with donor lymphocytes performed at the time of transplantation. The clinical outcome of a transplant is greatly influenced by the characteristics of the donor organ transplanted ${ }^{20}$, the immunological history of the recipient ${ }^{18}$, and the immunosuppressive regimen used. In our center, all potential recipients are investigated every 3 months for the presence of HLA antibodies by means of extensive screening protocols. All patients are transfused, and posttransfusion serum samples are investigated for the development of HLA antibodies by crossmatches with the specific blood donor. After every additional blood transfusion, the development of anti-HLA class I and II antibodies is studied. 
Table 5.5 Pretransplant recipient and donor characteristics in 76 renal allograft at risk (group 2) based on FC crossmatch result group".

\begin{tabular}{|c|c|c|c|}
\hline & & FCXM positieve $(n=21)$ & FCXM negative $(n=55)$ \\
\hline \multicolumn{2}{|c|}{ Recipient age (yr) } & $45.5 \pm 12.34$ & $45.7 \pm 12.17$ \\
\hline \multicolumn{2}{|c|}{ Donor age (yr) } & $33.9 \pm 14.32$ & $38.4 \pm 15.66$ \\
\hline \multicolumn{2}{|l|}{$\operatorname{Sex}(m / f)$} & $10 / 11$ & $27 / 28$ \\
\hline \multicolumn{2}{|l|}{ WIT (min) } & $33.3 \pm 9.71$ & $33.5 \pm 8.05$ \\
\hline \multicolumn{2}{|l|}{ CIT (hour) } & $29.4 \pm 6.90$ & $30.7 \pm 7.72$ \\
\hline \multirow{2}{*}{ PRA (\%) P } & Peak serum & $53.9 \pm 37.72$ & $51.1 \pm 34.81$ \\
\hline & Current serum & $34.8 \pm 34.15$ & $29.9 \pm 33.66$ \\
\hline \multirow{5}{*}{$\begin{array}{r}\text { Prak PRA } \\
\\
2 \\
5 \\
8 \\
8\end{array}$} & $-5 \%$ & 2 & 4 \\
\hline & $-20 \%$ & 6 & 10 \\
\hline & $.50 \%$ & 2 & 19 \\
\hline & $-84 \%$ & 3 & 6 \\
\hline & $-100 \%$ & 8 & 16 \\
\hline \multicolumn{2}{|c|}{ Number of transfusions } & $4.7 \pm 7.02$ & $7.1 \pm 16.16$ \\
\hline \multirow[t]{4}{*}{ Transplant no. } & 1 & 10 & 29 \\
\hline & 2 & 9 & 18 \\
\hline & 3 & 2 & 6 \\
\hline & 4 & 0 & 2 \\
\hline \multicolumn{4}{|c|}{ Number of mismatches } \\
\hline \multirow[t]{3}{*}{ HLA-A } & 0 & 6 & 23 \\
\hline & 1 & 10 & 24 \\
\hline & 2 & 5 & 8 \\
\hline \multirow[t]{3}{*}{ HLA-B } & 0 & 7 & 14 \\
\hline & 1 & 12 & 37 \\
\hline & 2 & 2 & 4 \\
\hline \multirow[t]{3}{*}{ HLA-DR } & 0 & 16 & 31 \\
\hline & 1 & 5 & 20 \\
\hline & 2 & 0 & 4 \\
\hline
\end{tabular}

" Group 2 comprised all patients at risk (1983-1992), sensitized or regrafted due to immunological failure of a previous graft within 1 year after transplant. Data are given as number or mean $\pm S D$. None of the comparisons between FC crossmatch result and characteristics of donor or recipient reached statistical significance by Mann-Whitney $U$ test, unpaired Student's $t$ test, or chi-square test.

The presence of autoantibodies is investigated using crossmatches with autologous patient lymphocytes for all serum samples obtained. HLA antigens are considered not acceptable for a patient if specific antibodies have been demonstrated either at the time of transplant or in the past. Mismatches from previous transplants are excluded, as are the paternally inherited antigens of the children in female patients. Matching priority for transplantation is HLA-DR, HLA-B, and HLA-A. All patients reveived transplants with a negative CDC crossmatch for current and historical sera. Using this policy, a 1-year graft survival rate of approximately $85 \%$ 
is accomplished.

We wanted to investigate whether performing FC crossmatches, which also detect non-complement binding and low-titer anti-HLA antibodies, would influence the clinical outcome of the transplants. Is the additional sensitivity of the FC crossmatch required in a patient population in which acceptable and unacceptable matches are well defined? Does performance of a more sensitive test not lead to a higher false-positive rate?

The results of our study showed that $13 \%$ of the CDC crossmatch-negative recipients have a positive FC crossmatch in the consecutive patient group (group 1). None of the clinical outcome parameters was significantly correlated with the FC crossmatch result. The effect of the FC crossmatch might be more clearly demonstrable in a patient population at risk, i.e., sensitized or retransplant recipients. We looked at the subgroup of patients in group 1 who fulfilled these criteria, but no effect could be demonstrated. However, the number of recipients at risk in group 1 was rather small; therefore, this number was extended by 29 sensitized and/or retransplant patients (group 2). The results from group 2 showed a higher percentage of positive FC crossmatches (28\%), but again no statistically significant correlation could be obtained for any of the clinical parameters tested.

Different protocols for performing FC crossmatches have been described ${ }^{2,6,8,11-13,21}$ ${ }^{24}$. In this study, FC crossmatches were performed as described by Scornik et al. ${ }^{13}$. Positivity was defined as fluorescence ratio between test and control serum. Antihuman IgG was tested at different concentrations and finally used at the concentration that gave optimal discrimination (diluted 1:16). Sensitivity was tested by diluting different positive sera, and an increased sensitivity was shown compared with $\mathrm{CDC}$, as measured by the number of dilution steps (three to six times). A similar increase was shown by Talbot et al. ${ }^{1}$. The nature of the antibody responsible for the positive FC crossmatch is not exactly known. Specific anti-class II antibodies were shown not to react in the FC protocol used. Therefore, class II antibodies were considered unlikely. Class 1 antibodies are the most likely candidates; however, to establish the involvement of specific anti-class I antibodies, blocking studies, as done by Karuppan et al. ${ }^{21,24}$, have to be performed. Since we did not carry out such experiments, we cannot exclude that some positive FC crossmatches are the result of non-HLA-directed activity.

The main reason for the lack of correlation between the result of FC crossmatches and graft outcome is probably the pretransplant protocol used in our center for recipients. Patient-donor combinations with incompatible HLA antigens are excluded from transplantation. In this study, we showed a 1-year graft survival rate 
of $91 \%$ in first transplant unsensitized recipients. For retransplants and sensitized recipients, this figure dropped to $80 \%$. This figure equals the 1-year graft survival rate reported for sensitized recipients transplanted with a negative FC crossmatch $^{12.25}$.

Since this is a retrospective study on immunologically well-documented patients, we cannot rule out that, prospectively, the FC crossmatch could have a beneficial effect on less well longitudinally studied recipients. Since FC is clearly a more sensitive technique ${ }^{1.2}$, it is possible that the effect of the FC crossmatch is considerable in patients in whom the HLA antibody history is unknown or less well known. It is possible that the real value of the FC crossmatch is confined to crossmatches for this patient group.

In conclusion, our data show that FC crossmatches in the consecutive group of recipients (group 1) are not superior to the classical negative CDC crossmatches with regard to clinical outcome, even though an additional $13 \%$ of the patients are found to be crossmatch positive with the FC technique. On the contrary, one could argue that transplantation with a mandatory negative FC crossmatch might have excluded a number of recipients from transplantation who in fact are doing very well. This holds for $18 \%$ of the group at risk when tested with current serum, and $28 \%$ if either current or peak sera are taken into account. 


\section{References}

1. Talbot D, Givan AL, Shenton BK, Stratton A, Proud G, Taylor RMR. Rapid detection of low levels of donor specific IgG by flow cytometry with single and dual colour fluorescence in renal transplantation. J Immunol Methods 1988; 112: 279-83.

2. Chapman JR, Deierhoi MH, Carter NP, Ting A, Morris PJ. Analysis of flow cytometry and cytotoxicity crossmatches in renal transplantation. Transplant Proc 1985: 17: 2480-1.

3. Garovoy MR. Flow cytometry crossmatch testing in renal transplantation. Transplant Immunol Lett 1988; 5: 1-2.

4. Talbot D, Givan AL, Shenton BK, Proud G, Taylor RMR. The nature of donorspecific IgG isotypes identified by flow cytometry in the preoperative crossmatch. Transplant Proc 1988; 20: S84-5.

5. Cook DJ, Terasaki PI, Iwaki Y, Terashita GA, Lau M. An approach to reducing early kidney transplant failure by flow cytometry crossmatching. Clin Transplant 1987: 1: 253-6.

6. Cook DJ, Terasaki PI, Iwaki Y, Terashita GY, Fujikawa J, Gera J, Takeda A, Danovitch G, Rosenthal JT, Fine R, Ettenger R, Mendez R, Dainko E, Kavalich A, Martin D, Teichman S, Ward H, Koyle M, Berne T, Brennan LP, Lieberman E, Cohen A, Strauss F. Flow cytometry crossmatching for kidney transplantation. In: Terasaki PI, ed. Clinical Transplants 1988. UCLA Tissue Typing Laboratory L.A. 1988: $375-80$.

7. Kerman RH, Van Buren CT, Lewis RM, DeVera V, Baghdahsarian V, Gerolami K, Kahan BD. Improved graft survival for flow cytometry and antihuman-globuline crossmatch negative retransplantation recipients. Transplantation 1990; 49: 52-6.

8. Talbot D, Givan AL, Shenton BK, Stratton A, Proud G, Taylor RMR. The relevance of a more sensitive cross match-assay to renal transplantation. Transplantation 1989; 47: 552-5.

9. Thistlethwaite JR, Buckingham M, Stuart JK, Gaber AO, Mayes JT, Stuart FP. Tcell immunofluorescent flow cytometry crossmatch results in cadaver donor renal transplantation. Transplant Proc 1987; 19: 722-4.

10. Ogura K. Terasaki PI, Johnson C, Mendez R, Rosenthal JT, Ettenger R, Martin DC, Dainko E, Cohen L, Mackett T, Berne T, Barba L, Lieberman E. The significance of a positive flow cytometry crossmatch test in primary kidney transplantation. Transplantation 1993: 56: 294-8.

11. Talbot D, Cavanagh G, Coates E, Givan AL, Shenton BK, Lennard TWJ, Proud G, Taylor RMR. Improved graft outcome and reduced complications due to flow cytometric crossmatching and DR matching in renal transplantation. Transplantation 1992: 53: 925-8.

12. Kerman RH, Kimball PM, van Buren CT, Lewis R $<$ DeVera V, Baghdahsarian V, Heydari A, Kahan BD. AHG and DTE/AHG procedure identification of crossmatchappropriate donor-recipient pairings that result in improved graft survival. Transplantation 1991; 51: 316-20. 
13. Scornik JC, Brunson ME, Schaub B, Howard RJ, Pfaff WW. The crossmatch in renal transplantation: evaluation of flow cytometry as a replacement for standard cytotoxicity. Transplantation 1994: 57: 621-5.

14. Kahan BD, Grevel J. Optimization of cyclosporine therapy in renal transplantation by a pharmacokinetic strategy. Transplantation 1988; 46: 631-44.

15. Iwaki Y, Terasaki PI. Primary nonfunctioning in human cadaveric kidney transplantation: evidence for hidden acute rejection. Clin Transplant 1987; 1: 125.

16. Dafoe DC, Bromberg JS, Grossman RA, Tomaszewski JE, Zmijewski CM, Perloff L, Maji A, Asplund MW, Alfrey EJ, Sack M, Zellers L, Kearns J, Barke CF. Renal transplantation despite a positive antiglobulin crossmatch with and without prophylactic OKT3. Transplantation 1991; 51: 762-8.

17. Scornik JC, Salomon DR, Lim PB, Howard RJ, Pfaff WW. Posttransplant antidonor antibodies and graft rejection: evaluation by two-color flow cytometry. Transplantation 1989; 47: 287-90.

18. Terasaki PI, Mickey MR. Cecka M. Iwaki Y. Cicciarelli J. Clinical kidney transplants 1988. Immunol Lett 1989; 21: 33-8.

19. Thorogood J, van Houwelingen JC, Persijn GG, van Rood JJ. The contribution of failure time of first kidney graft to predicting failure of regrafts. Transplant Proc 1990; 22: 1915-6.

20. Cecka JM, Terasaki PI. Improvement of kidney transplant regraft results by using trauma death donors. Transplantation 1987; 44: 792-5.

21. Karuppan SS, Ohlman S, Möller E. The occurrence of cytotoxic and noncomplement-fixing antibodies in the crossmatch serum of patients with early acute rejection episodes. Transpantation 1992; 54: 839-43.

22. Lazda VA, Pollak R, Mozes MF, Jonasson $\mathrm{O}$. The relationship between flow cytometer crossmatch results and subsequent rejection episodes in cadaver renal allograft recipients. Transplantation 1988; 45: 562-5.

23. Mahoney RJ, Ault KA, Given SR, et al. The flow cytometric crossmatch and early renal transplant loss. Transplantation 1990; 49: 527-35.

24. Karuppan SS, Lindholm A, Möller E. Fewer acute rejection episodes and improved outcome in kidney-transplanted patients with selection criteria based on crossmatching. Transplantation 1992; 53: 666-73.

25. Berteli AJ, Daniel V, Möhring K, Staehler G, Opelz G. Association of kidney failure with a positive flow cytometric crossmatch. Clin Transplant 1992; 6: 31-4. 


\section{Chapter 6}

\section{Donor-specific antibodies after}

transplantation by flow cytometry. Relative change in fluorescence ratio most sensitive risk factor for graft survival

MHL Christiaans, R Overhof-de Roos, F Nieman, JP van Hooff, EM van den Berg-Loonen

Transplantation 1998; 65: 427-33 


\section{Abstract}

\section{Background}

There is no consensus on the role of donor-directed antibodies after renal transplantation detected by complement-dependent-cytotoxicity (CDC) or by flow cytometry (FC).

\section{Methods}

Therefore, antibody formation was studied by FC and correlated with clinical course in a group of patients who received transplants between 1983 and 1993. All had a negative current CDC crossmatch and were treated with cyclosporine. Current and posttransplant sera from 143 donor-recipient combinations were studied retrospectively. Antibodies were considered present in FC if the fluorescence ratio between serum and negative control was $>2.65$.

\section{Results}

Of 143 patients, $17(11.9 \%)$ were found to be positive in the posttransplant FC crossmatch and $126(88.1 \%)$ were negative. Of the positive patients, 3 were already positive in the current FC crossmatch, whereas 14 demonstrated a positive posttransplant FC crossmatch after a negative current FC crossmatch. It was noteworthy that, from 16 patients with a positive current $\mathrm{FC}$ crossmatch, 13 turned negative in the posttransplant crossmatch. In 113 recipients $(79 \%)$, both pre- and posturansplant FC crossmatches were negative. The development of a positive FC crossmatch after transplantation was a significant risk factor for graft survival in Cox-regression analysis $(P=0.01)$.

The results were also studied as relative change in fluorescence ratio (RCFR). RCFR was determined by classifying the recipients in quartiles according to their change in flow cytometric value from current to posttransplant serum. Quartiles were defined as follows: quartile 1 , decrease $>10 \%$; quartile 2 , decrease $0-10 \%$; quartile 3 , increase $>0-30 \%$; and quartile 4 , increase $>30 \%$. RCFR proved to be the only significant risk factor for graft survival (odds ratio for quartile 4 vs. quartile $1,3.27 ; P<0.02$ ). More rejections were shown for increasing quartile numbers $(P<0.001)$.

\section{Conclusions}

Classification of patients by RCFR detected more patients with unfavorable clinical outcome $(25 \%$ vs. $11 \%)$ than by FC crossmatch. 


\section{Introduction}

Development of antibodies directed against the kidney donor after transplantation is related with a higher incidence of rejection and lower graft survival. Martin et al.' showed that cytotoxic antibodies developed after transplantation were usually donor-directed HLA class I antibodies. Antibody positive patients had a lower graft survival compared with patients without so-called panel reactive antibodies (PRA). Lobo et al. ${ }^{2}$ demonstrated that the occurrence of donor-directed cytotoxic antibodies at the time of rejection was a bad prognostic sign for 1-year graft survival. At the time of rejection, antibodies were detected in $23 \%$ of the patients and their 1-year graft survival was $26 \%$. Patients with rejection but without detectable antibodies had a 1-year graft survival of $72 \%$. Rejection-free patients had no antibodies and had a functioning graft at 1 year.

Flow cytometry (FC) is a more sensitive method, which detects complement-fixing antibodies as well as non-complement-fixing antibodies ${ }^{3-6}$. Data are available showing that FC more often detects patients that have developed antibodies after transplantation. Scornik et al. ' showed donor-directed antibodies to be present after transplantation in $27 \%$ of the patients with rejection versus $0 \%$ in the nonrejectors. In approximately half of the patients, these antibodies were detectable before rejection was clinically diagnosed. Recently, Utzig et al. ${ }^{8}$ demonstrated that half of the patients who developed antibodies after transplantation experienced rejection, whereas, in Ab-negative (Abneg) patients, this was only $15 \%$.

One of the major drawbacks of the FC technique is the lack of standardization. The definition of positive and negative test results varies considerably between different investigations. Usually, discrimination between positive and negative results is based on reaction of a 'third-party' negative control serum with donor lymphocytes from different individuals. When monitoring patients after transplantation, a different approach can be used. Development of donor-directed antibodies might be assessed by direct comparison of differences in reactivity of various sera of the same recipient against donor cells. In that way, smaller differences in reactivity against donor cells might be detected. The purpose of the present study was to investigate the effect of the posttransplant FC crossmatch on clinical outcome in renal transplant recipients.

\section{Materials and Methods}

\section{Patients}

All patients included received a cadaveric kidney graft in our center between 
January 1983 and December 1992 and have been described previously ${ }^{10}$. Lymphocytes were available from 143 donor-recipient combinations. The patients were treated with cyclosporine (CsA), and their characteristics are presented in Table 6.1.

Transplants were performed under the auspices of Eurotransplant. Matching priority was HLA-DR, HLA-B, and HLA-A. All patients had negative class I crossmatches $(\mathrm{NIH})$ at the time of transplantation with current as well as Ab-positive (Abpos) historical samples (peak sera). Current sera were drawn at the time of transplantation. Posttransplant sera were obtained after rejection or within 6 months after transplantation if no rejection-episode occurred. In case the kidney graft had to be removed, blood samples were drawn 2-4 weeks later. Sera drawn within 10 days after the last gift of OKT3 were excluded to avoid false positive results $^{11}$. Crossmatches were performed using liquid-nitrogen-stored spleen lymphocytes of the kidney donors.

Table 6.1 Pretransplant recipient and donor characteristics".

$\begin{aligned} & \text { Recipient age (yr) } \\ & \text { Donor age (yr) } \\ & \text { Gender }(\mathrm{m} / \mathrm{f})\end{aligned}$
WIT (min)
CIT (hour)
PRA (\%) Peak serum
$\quad$ Current serum
Current/peak PRA $\quad 0-5 \%$
$\quad 6-84 \%$
$\quad 85-100 \%$

Number of transfusions

Transplant no.

1
2
3
4

Number of mismatches

$$
\begin{array}{ll}
\text { HLA-A } & 0 / 1 / 2 \\
\text { HLA-B } & 0 / 1 / 2 \\
\text { HLA-DR } & 0 / 1 / 2
\end{array}
$$

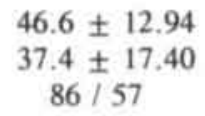

$96 / 73$

$38 / 46$

$9 / 24$

$5 \pm 10.5$

102

30

9

2

\footnotetext{
" Data are given as numbers or mean $\pm S D$.
} 


\section{Immunosuppression}

The immunosuppressive regimen consisted of CsA and low-dose prednisolone (PRED; $10 \mathrm{mg} /$ day) for recipients of first grafts. The CsA was started intravenously before surgery (continuous infusion; $4 \mathrm{mg} / \mathrm{kg} / 24 \mathrm{hr}$ ) and switched orally ( $4 \mathrm{mg} / \mathrm{kg}$ b.i.d.) at day 2 . The PRED dose was diminished to $7.5 \mathrm{mg} /$ day at month 1 and to $5 \mathrm{mg} / \mathrm{day}$ at month 3 . For recipients without rejection, the PRED dose was further tapered to $0 \mathrm{mg}$ in the next months. For highly immunized recipients (PRA $>85 \%$ ) and retransplant recipients, azathioprine (AZA, $\pm 1 \mathrm{mg} / \mathrm{kg}$ body weight) was added to the above-described regimen, and the dose of PRED was tapered to $5 \mathrm{mg}$. The CsA level was determined in whole blood by highperformance liquid chromatography or by monoclonal radioimmunoassay. The trough CsA level for CsA+PRED recipients was $0.15-0.20 \mathrm{mg} / \mathrm{L}$ in the first 3 months after transplantation and $0.10-0.15 \mathrm{mg} / \mathrm{L}$ after 3 months. For CsA + PRED + Aza recipients, the trough CsA level was $0.10-0.15 \mathrm{mg} / \mathrm{L}$ in the first 3 months after transplantation and $0.05-0.10 \mathrm{mg} / \mathrm{L}$ after 3 months.

\section{Clinical outcome parameters}

Rejection was defined as any rejection treatment in the first 6 months after grafting. In general, rejections were proven by needle core biopsy. Rejection treatment consisted of a 10-day course of rabbit anti-lymphocyte globulin (RIVM, Bilthoven, the Netherlands) or OKT3-Orthoclone (Jansen Cilag, Tilburg, the Netherlands). For patients who had received rabbit anti-lymphocyte globulin in the past or who had a seriously compromised cardiovascular system, the rejection was treated with three doses of methylprednisolone $(0.5-1.0 \mathrm{~g} /$ dose $)$.

Graft failure was defined as loss of kidney function but also included death of recipient with functioning graft. No exclusions were made. Graft failure was also analysed with censoring death with functioning graft in the Cox-regression analysis as stated in the text.

All patients were followed up until January 1, 1996, or until their date of death.

\section{Crossmatches}

Crossmatches performed included two complement-dependent crossmatches: the standard NIH crossmatch and the dithiothreitol (DTT) crossmatch in which the serum is treated with dithiotreitol to reduce IgM antibodies. Pre- and posttransplant sera were tested by both complement-dependent techniques as well as by FC using liquid-nitrogen-stored spleen lymphocytes of the kidney donor. In NIH, presence of donor-directed antibodies in the recipient serum was defined as cell death $>20 \%$ above background. If DTT treatment decreased cell death to less than $20 \%$ above background, donor-specific antibodies of the $\mathrm{IgG}$ type were considered absent. 


\section{FC}

The FC crossmatch technique was performed as described previously ${ }^{10}$. In short, $10^{5}$ lymphocytes were incubated with $50 \mu \mathrm{L}$ of serum for $30 \mathrm{~min}$ at $21^{\circ} \mathrm{C}$. After washing, $20 \mu \mathrm{L}$ of a fluorescein-conjugated $\mathrm{F}(\mathrm{ab}){ }^{\prime 2}$ goat anti-human IgG (Tago, Burlingame, CA) diluted 1:16 were added, as well as $5 \mu \mathrm{L}$ of phycoerythrinconjugated anti-CD3 mouse monoclonal Ab (Dakopatts, Glostrup, Denmark) and incubated for $30 \mathrm{~min}$ at $4^{\circ} \mathrm{C}$. Finally, cells were washed twice and resuspended in $250 \mu \mathrm{L}$ of phosphate-buffered saline. For all donor crossmatches, spleen lymphocytes were incubated with (1) the recipient's current serum, (2) the recipient's posttransplant serum, (3) a positive control serum, and (4) a negative control serum. The positive control was an allo-antiserum routinely used in our laboratory with $100 \%$ PRA. The negative control was pooled serum from 20 healthy male blood bank donors. Flow cytometric analysis was performed with a FACScan flow cytometer (Becton Dickinson, 256 channels, logarithmic scale). Routine instrument function quality control included calibration by test beads and fluorescence compensation (Calibrite; Becton Dickinson). From each sample, 10,000 cells were analyzed in the lymphocyte gate. From the $\mathrm{CD}^{+}$cells, a histogram of fluorescein isothiocyanate (logarithmic scale) was made. The median channel of fluorescence was calculated.

Crossmatch results were expressed as the fluorescence ratio between median channel of recipient serum and negative control. The presence of donor-directed antibodies by FC was defined by comparing the fluorescence ratio of patient serum to the fluorescence ratio of negative control sera (mean $=1.185, \mathrm{SD}=0.487$ ) as described by Scornik et al. ${ }^{7}$. The cutoff point for presence or absence of antibodies was set at 2.65 (mean+3SD). Antibodies were regarded as present if the ratio of the median channel of the patient serum to the negative control serum was $\geq 2.65$, provided the ratio of positive control to negative control was $\geq 2.65$. Antibodies were regarded as absent if the ratio of the median channel of the patient serum to the negative control was $<2.65$, provided the ratio of positive control to negative control was $\geq 2.65$.

Presence of donor-specific antibodies was also defined by the relative change in fluorescence ratio (RCFR) of the posttransplant serum compared with the fluorescence ratio of the pretransplant serum. RCFR was defined as the median channel of fluorescence of postserum minus preserum divided by preserum times $100 \%$. An increase in fluorescence ratio is considered an indication for the presence of donor-specific antibodies. 


\section{Statistics}

Statistical analysis was performed using SPSS for Windows version 6.1. The proportional hazards regression analysis (Cox regression) was performed by both stepwise forward and backward selection techniques for all suspected risk factors except the result of the FC technique, which was always included in each model. In case rejection was the outcome parameter, the suspected risk factors tested for included age of recipient, gender, current PRA, transplant number, number of HLA ABDR mismatches, and age of donor. Current PRA was classified according to Eurotransplant criteria, i.e., nonimmunized (NI), immunized (I), and highly immunized (HI). Subsequently, a basic model was tested for, which included only direct risk odds ratio effects that were statistically significant. Thereafter, all interaction terms were introduced separately into the basic model. With graft survival as outcome parameter, rejection was included as a risk factor in the model. The same policy in model selection was followed for the outcome variable. Parametric (independent groups Student's $t$-test) and nonparametric tests (Pearson chi-square test, Fisher's exact test, Kruskal-Wallis test) were performed when indicated. A $P$-value $<0.05$ was considered to be statistically significant.

\section{Results}

\section{Antibodies (CDC) posttransplant and clinical outcome}

Posttransplant sera of 143 patients were tested for the presence of class I antibodies directed against the donor by performing CDC crossmatches (NIH, DTT) with spleen lymphocytes of their kidney donors. For 12 of the donors, the viability of the lymphocytes was insufficient to perform a reliable CDC crossmatch. Of the remaining 131 patients, $8(6.1 \%)$ were shown to be Abpos, whereas, in 123 (93.9\%) patients, no antibodies could be detected (Abneg). Abpos recipients had a significantly lower 1-year graft survival rate compared with Abneg recipients: $37.5 \%$ versus $86.2 \%$ (Fisher's exact test; $P=0.0035$ ). All 8 Abpos patients were treated for rejection $(100 \%)$ versus 46 of the 123 Abneg patients $(37.4 \%$ ) (Fisher's exact test; $P=0.0006$ ). Rejection was diagnosed at a median of 7 days after transplantation for Abpos patients (range, $0-100$ ) and 9 days after transplantation for Abneg patients (range, 1-136) (Kruskal-Wallis test, $\chi^{2}=0.238,1 \mathrm{df} ; P=0.63$ ).

Both groups did not differ significantly for pretransplant characteristics (age of donor, age and gender of recipient, peak and current PRA grade, warm ischemia time, cold ischemia time, number of transfusions, transplant number, number of HLA-ABDR mismatches). The posttransplant serum was drawn 100.5 days after transplantation (median; range, 10-166) for Abpos patients and 40 days (median; 
range, 2-164) for Abneg patients (Kruskal-Wallis test $\chi^{2}=1.15,1 \mathrm{df} ; P=0.28$ ).

\section{Antibodies (FC) posttransplant and clinical outcome}

Posttransplant sera of all patients were tested for the presence of antibodies directed against the donor by FC crossmatches. Of 143 patients, 17 (11.9\%) were found to be positive and $126(88.1 \%)$ were negative.

In 16 recipients $(11.2 \%)$, a pretransplant positive FC crossmatch had been demonstrated (Abprepos group). Of these patients, 3 remained positive in the posttransplant FC crossmatch, whereas 13 turned negative. Fourteen recipients $(9.8 \%)$ demonstrated a positive posttransplant FC crossmatch after a negative current FC crossmatch (Abpostpos group), whereas in 113 recipients (79\%) both pre- and posttransplant FC crossmatches were negative (Abneg group).

\section{Graft survival}

Cox-regression analysis of risk factors (Ab group, age of recipient, gender, current PRA, the occurrence of rejection, transplant number, number of HLA-ABDR mismatches, and age of donor) revealed the $\mathrm{Ab}$ group to be the only risk factor contributing to graft survival $(P=0.051,2 \mathrm{df})$. The Abpostpos group had an odds ratio of $2.25(95 \% \mathrm{CI}: 1.17-4.32)$ compared with the Abneg group (Table 6.2). The 5-year graft survival rates for Abpostpos recipients and Abneg recipients were $35 \%$ and $60 \%$, respectively (Figure 6.1 ). When patients who died with functioning graft were censored, graft survival rates were significantly lower in Abpostpos recipients compared with the other groups $(P<0.01,2 \mathrm{df})$ (Table 6.2).

\section{Rejection}

Cox regression analysis of risk factors (Ab group, age of recipient, gender, current PRA, transplant number, number of HLA-ABDR mismatches, and age of donor) revealed current PRA $(P=0.005,2 \mathrm{df})$, number of HLA-DR mismatches ( $\mathrm{O}$ vs. $\geq 1, P<0.01)$, gender $(P=0.02)$, and $\mathrm{Ab}$ group $(P<0.001,2 \mathrm{df})$ to contribute significantly to incidence of rejection. Patients with antibodies after transplant had a significant higher rejection rate compared with the other groups $(P=0.0001)$. Rejection rates were $80 \%, 25 \%$, and $35 \%$ for Abpostpos, Abprepos, and Abneg patients, respectively (Table 6.3 and Figure 6.2). The number of days between date of rejection and date of serum sample was not significantly different between the three groups (median number of days (range): 16 (7-105), 59 (-62 to 159), and 24 (-81 to 139$)$, respectively) (Kruskal-Wallis test: $\chi^{2}=4.24,2 \mathrm{df} ; P=0.12$ ). 
Table 6.2 Graft survival based on FC crossmatch result.

\begin{tabular}{lcccc}
\hline Risk factor & Odds ratio & $(95 \% \mathrm{Cl}$ range $)$ & df & $P$ \\
\hline Death with functioning graft included in graft loss & & & & \\
FCXM (all) & 2.25 & $(1.17-4.32)$ & 1 & 0.051 \\
FCXM (2 vs. 1) & 1.19 & $(0.56-2.50)$ & 1 & 0.653 \\
FCXM (3 vs. 1) & 0.53 & $(0.21-1.31)$ & 1 & 0.169 \\
FCXM (3 vs. 2) & & & & \\
Death with functioning graft censored & & & & \\
FCXM (all) & 3.11 & $(1.53-6.32)$ & 1 & 0.007 \\
FCXM (2 vs. 1) & 1.14 & $(0.45-2.94)$ & 1 & 0.779 \\
FCXM (3 vs. 1) & 0.37 & $(0.13-1.08)$ & 1 & 0.068 \\
FCXM (3 vs. 2) & & & & \\
\hline
\end{tabular}

2 Cox regression analysis in 143 recipients: suspected risk factors are recipient age, donor age, current PRA, transplant number, number of HLA-ABDR mismatches, occurrence of rejection, and gender. The final model results are shown including FC crossmatch result as the only statistically significant risk factor. Risk factors with more than two categories are broken down to indicator contrasts. FCXM $1=$ negative pretransplant, negative posttransplant; FCXM $2=$ negative pretransplant, positive posttransplant; FCXM $3=$ positive pretransplant, negative or positive posttransplant.

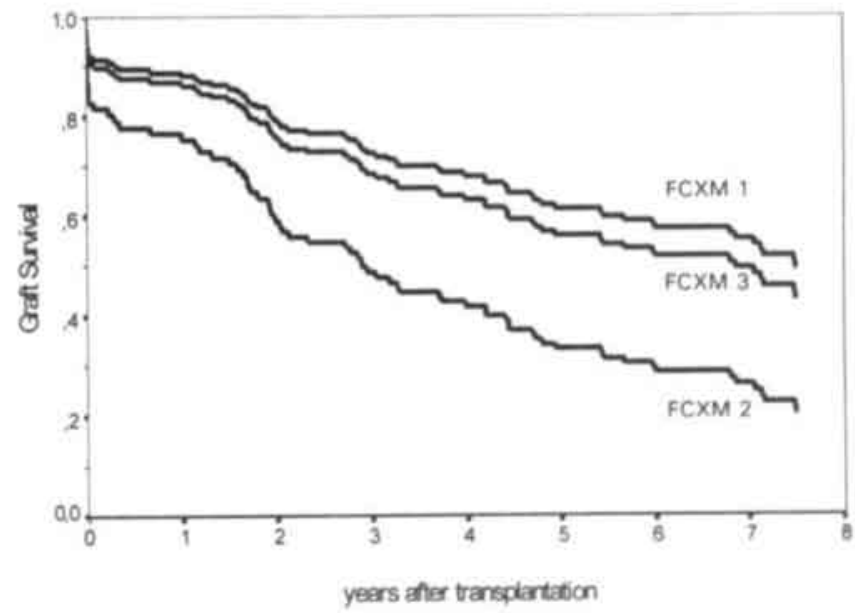

Figure 6.1 Predicted graft survival based on FC crossmatch result with risk factor FC crossmatch result. Cox regression model plot by risk factor FC crossmatch result. FCXM 1, patients with a negative pretransplant, negative posttransplant crossmatch $(\mathrm{n}=113)$; FCXM 2 patients with a negative pretransplant, positive posttransplant crossmatch $(n=14)$ : FCXM 3, patients with a positive pretransplant, negative or positive posttransplant crossmatch $(n=16)$. 


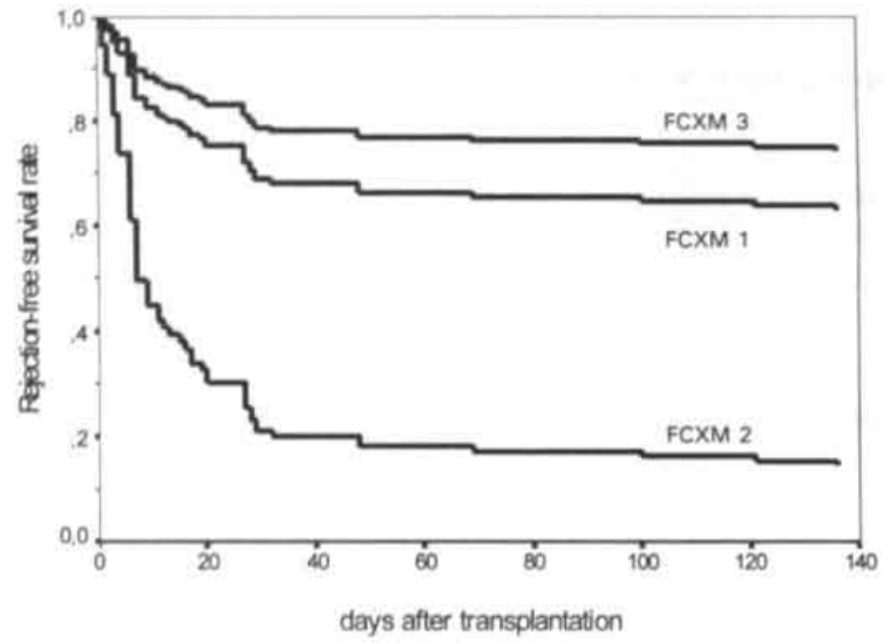

Figure 6.2 Predicted rejection-free survival based on FC crossmatch result. Cox regression model plot by FC crossmatch result with risk factors gender, current PRA, and number of DR mismatches. FCXM I, patients with a negative pretransplant, negative posttransplant crossmatch $(n=113)$; FCXM 2, patients with a negative pretransplant, positive posttransplant crossmatch $(n=14)$; FCXM 3, patients with a positive pretransplant, negative or positive posttransplant crossmatch $(n=16)$. Category survival curves are corrected for gender, number of DR mismatches and current PRA (classified according to Eurotransplant definition).

Table 6.3 Rejection based on FC crossmatch result".

\begin{tabular}{lcccc}
\hline Risk factor & Odds-ratio & $(\mathbf{9 5 \%}$ Cl range $)$ & df & $P$-value \\
\hline FCXM (all) & & -18 & 2 & 0.001 \\
FCXM (2 vs. 1) & 4.18 & $(2.06-8.49)$ & 1 & 0.001 \\
FCXM (3 vs. 1) & 0.64 & $(0.25-1.62)$ & 1 & 0.348 \\
FCXM (3 vs. 2) & 0.15 & $(0.05-0.48)$ & 1 & 0.001 \\
Mismatch & 2.04 & $(1.20-3.48)$ & 1 & 0.009 \\
HLA-DR ( $\geq$ I vs. 0) & 1.88 & $(1.10-3.22)$ & 1 & 0.022 \\
Gender (female) & & & & \\
Current PRA (all) & 1.57 & $(0.85-2.88)$ & 1 & 0.001 \\
Current PRA (I vs. NI) & 6.75 & $(2.57-17.76)$ & 1 & 0.151 \\
Current PRA (HI vs. NI) & 4.32 & $(1.65-11.31)$ & 1 & 0.001 \\
Current PRA (HI vs. I) & & & & 0.003 \\
\hline
\end{tabular}

- Cox regression analysis in 143 recipients: suspected risk factor are recipient age, donor age, current PRA, transplant number, number of HLA-ABDR mismatches, and gender. The final model results are shown including FC crossmatch result, number of DR mismatches, and current PRA as statistically significant risk factors. Risk factors with more than two categories are broken down to indicator contrasts. FCXM $I=$ negative pretransplant, negative posttransplant; FCXM $2=$ negative pretransplant, positive posttransplant; FCXM $3=$ positive pretransplant, negative or positive posttransplant.

- PRA classification according to Eurotransplant: $\mathrm{NI}=$ nonimmunized $(0-5 \%): \mathrm{I}=$ immunized $(6-84 \%) ; \mathrm{HI}=$ highly immunized $(>85 \%)$. 


\section{Antibodies posttransplant defined as RCFR and clinical outcome}

\section{$R C F R$}

The difference between current and posttransplant FC crossmatch value was expressed as the RCFR. An increase in the RCFR was shown in 74 patients; for the remaining patients the ratio was unchanged or decreased. The RCFR ranged from -85 to 1000 with a median of 3 . Because of the skewed distribution, log transformation of the data was performed. In the Cox regression analysis, these log-transformed RCFR proved to contribute significantly to graft survival $(P=0.0043)$ and rejection $(P=0.0026)$. To locate the caesura for increased risk, patients were classified into 4 groups (quartiles) according to their change in fluorescence ratio after grafting compared with pregrafting. The groups were approximately equal in size. Patients in quartile $1(n=35)$ had a decrease of $>10 \%$ in fluorescence ratio between pre- and posttransplant crossmatch result. Patients in quartile $2(\mathrm{n}=34)$ had a decrease from $<10 \%$ to $0 \%$, and patients in quartile 3 $(n=38)$ showed an increase from $0-30 \%$, whereas patients in quartile $4(n=36)$ had an increase $>30 \%$. The size of the groups was sufficiently large for application of Cox regression analysis.

\section{Graft survival}

Cox regression analysis of risk factors (quartile, age of recipient, gender, current PRA, occurrence of rejection, transplant number, number of HLA-ABDR mismatches, and age of donor) revealed that the quartile was the only significant risk factor contributing to graft survival $(P=0.014)$. Patients in quartile 4 had a significant lower graft survival rate compared with patients in quartile 1 (odds ratio for graft loss quartile 4 vs. quartile 1: 3.27 (95\% CI: 1.56-6.85). Differences between the other quartiles were not statistically significant (Table 6.4). Patients in quartile 1 had a 5-year graft survival rate of $76 \%$, whereas in quartile 2 and 3 this was $59 \%$ and in quartile $434 \%$ (Figure 6.3). When patients who died with functioning graft were censored, the patients in quartile 4 had a statistically significant lower graft survival rate compared with patients in quartile $1+2$ $(P=0.04)$ (Table 6.4). 


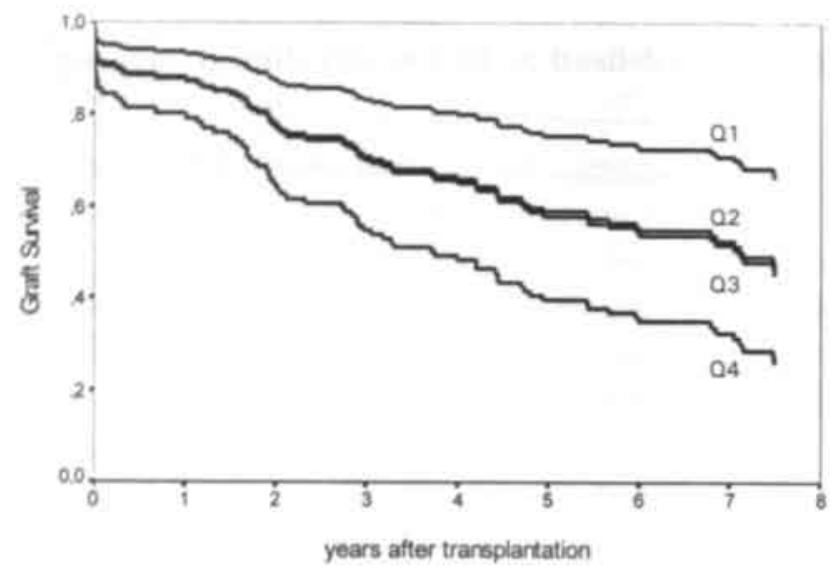

Figure 6.3 Predicted graft survival based on relative change in fluorescence ratio (RCFR). Cox regression model plot by risk factor RCFR. Quartiles are defined as follows: Q1. decrease $>10 \%(n=35) ; Q 2$, decrease $0-10 \%(n=34) ; Q 3$, increase $<30 \%(n=38)$; Q4, increase $>30 \%(\mathrm{n}=36)$.

Table 6.4 Graft survival based on relative change in fluorescence ration (RCFR).

\begin{tabular}{lcccc}
\hline Risk factor & Odds ratio & $\mathbf{( 9 5 \%}$ Cl range) & df & $P$ \\
\hline Death with functioning graft included in graft loss & & & & \\
Quartiles (all) & 1.87 & $(0.86-4.10)$ & 1 & 0.116 \\
Quartiles (2 vs. I) & 1.93 & $(0.90-4.13)$ & 1 & 0.089 \\
Quartiles (3 vs. 1) & 3.27 & $(1.56-6.85)$ & 1 & 0.002 \\
Quartiles (4 vs. 1) & 1.03 & $(0.54-1.97)$ & 1 & 0.926 \\
Quartiles (3 vs. 2) & 1.75 & $(0.94-3.26)$ & 1 & 0.079 \\
Quartiles (4 vs. 2) & 1.69 & $(0.93-3.07)$ & 1 & 0.082 \\
Quartiles (4 vs. 3) & & & & \\
Death with functioning graft censored & & & & \\
Quartiles (all) & 1.09 & $(0.41-2.92)$ & 1 & 0.857 \\
Quartiles (2 vs. 1) & 1.92 & $(0.82-4.48)$ & 1 & 0.133 \\
Quartiles (3 vs. 1) & 2.66 & $(1.14-6.23)$ & 1 & 0.024 \\
Quartiles (4 vs. 1) & 1.75 & $(0.75-4.10)$ & 1 & 0.196 \\
Quartiles (3 vs. 2) & 2.43 & $(1.04-5.70)$ & 1 & 0.041 \\
Quartiles (4 vs. 2) & 1.39 & $(0.69-2.78)$ & 1 & 0.355 \\
\hline Quartiles (4 vs. 3) & & &
\end{tabular}

- Cox regression analysis in 143 recipients; suspected risk factors recipient age, donor age, current PRA, transplant number, number of HLA-ABDR mismatches, occurrence of rejection, and gender. The final model results are shown including FC crossmatch result as the only statistically significant risk factor. Risk factors with more than two categories are broken down to indicator contrasts.

- RCFR classified as quartiles 1 through 4: Q1, decrease $>10 \%:$ Q2, decrease 0-10\%: Q3. increase < $30 \%$ : $Q 4$, increase $>30 \%$. 


\section{Rejection}

Current PRA ( $P<0.0001,2 \mathrm{df}$ ), the number of mismatches for HLA-DR ( $\mathrm{O}$ vs. $\geq 1 ; P=0.02)$, gender $(P<0.01)$, and quartile $(P<0.0001,3 \mathrm{df})$ contributed significantly to rejection as shown by Cox-regression analysis of risk factors (quartile, age of recipient, gender, current PRA, transplant number, number of HLA-ABDR mismatches, and age of donor). Patients in quartile 4 had a significantly higher rejection rate $(75 \%)$ compared with all other quartiles. Quartiles 2 and 3 had an almost equal rate of $30 \%$, whereas quartile 1 showed $15 \%$ rejection. A trend toward more rejections was noticed for increasing quartile numbers; however, the differences between quartiles 1, 2, and 3 were not statistically significant (Table 6.5 and Figure 6.4).

Table 6.5 Rejection based on relative change in fluorescence ratio (RCFR)

\begin{tabular}{lcccc}
\hline Risk factor $^{*}$ & Odds ratio & (95\% Cl range) & df & $\boldsymbol{P}$ \\
\hline Quartiles (all) & & & 3 & 0.001 \\
Quartiles (2 vs. 1) & 2.16 & $(0.88-5.30)$ & 1 & 0.092 \\
Quartiles (3 vs. 1) & 2.07 & $(0.80-5.32)$ & 1 & 0.132 \\
Quartiles (4 vs. 1) & 7.00 & $(2.86-17.16)$ & 1 & 0.001 \\
Quartiles (3 vs. 2) & 0.96 & $(0.42-2.18)$ & 1 & 0.915 \\
Quartiles (4 vs. 2) & 3.24 & $(1.56-6.73)$ & 1 & 0.002 \\
Quartiles (4 vs. 3) & 3.39 & $(1.61-7.13)$ & 1 & 0.001 \\
Mismatch HLA-DR ( $\geq 1$ vs. 0) & 1.87 & $(1.10-3.19)$ & 1 & 0.021 \\
Gender (female) & 2.16 & $(1.23-3.78)$ & 1 & 0.007 \\
Current PRA (all) & - & - & 2 & 0.001 \\
Current PRA (I vs. NI) & 2.32 & $(1.21-4.44)$ & 1 & 0.011 \\
Current PRA (HI vs. NI) & 9.10 & $(3.59-23.09)$ & 1 & 0.001 \\
Current PRA (HI vs. I) & 3.92 & $(1.57-9.78)$ & 1 & 0.003 \\
\hline
\end{tabular}

- Cox regression analysis in 143 recipients: suspected risk factors recipient age, donor age, current PRA, transplant number, number of HLA-ABDR mismatches, and gender. The final model results are shown including FC crossmatch result, number of DR mismatches, gender, and current PRA as statistically significant risk factors. Risk factors with more than two categories are broken down to indicator contrasts.

RCFR classified as quartiles 1 through 4: Q1, decrease $>10 \% ; \mathrm{Q}$, decrease $0-10 \% ; \mathrm{Q}$, increase $<30 \% ; \mathrm{Q} 4$, increase $>30 \%$.

"PRA classification according to Eurotransplant: $\mathrm{NI}=$ non immunized $(0-5 \%) ; \mathrm{I}=$ immunized $(6$ $84 \%) ; \mathrm{HI}=$ highly immunized $(>85 \%)$. 


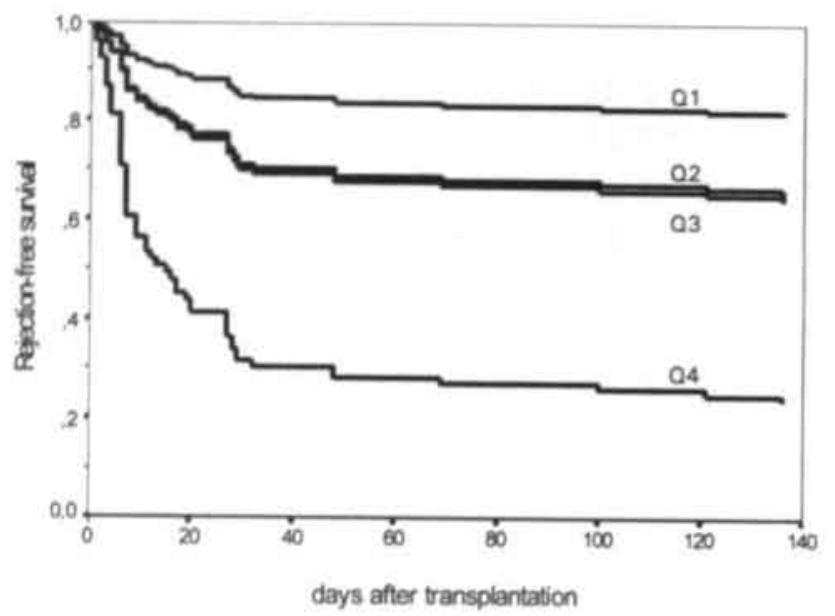

Figure 6.4 Predicted rejection-free survival based on relative change in fluorescence ratio (RCFR). COX regression model plot by RCFR with risk factors gender, current PRA, and number of DR mismatches. Quartiles are defined as follows: Q1, decrease $>10 \%(\mathrm{n}=35) ; \mathrm{Q}$, decrease $0-10 \% \quad(\mathrm{n}=34) ; \mathrm{Q} 3$, increase $<30 \%(\mathrm{n}=38) ; \mathrm{Q}$, increase $>30 \%(n=36)$. Category survival curves are corrected for gender, number of DR mismatches, and current PRA (classified according to Eurotransplant definition).

The interaction term between gender and quartile turned out to be statistically significant $(P=0.0014,3 \mathrm{df})$. Separate analysis for male patients could not be performed due to size of patient population. In female patients, it enhances the discriminating effect by showing an additional statistical difference between quartile 1 and higher quartiles (quartile 1-2: $P=0.02$, quartile 1-3: $P=0.0001$, quartile 1-4: $P=0.0089$ ). The differences between quartile 4 and lower quartiles were maintained.

The number of days between date of rejection and date of serum sample did not differ significantly among the four groups (median number of days (range) for quartile 1: 49 (-62 to 139), for quartile $2: 18.5$ (-81 to 126), for quartile $3: 20$ $(10-83)$, and for quartile 4: $20(7-159)$ (Kruskal-Wallis test: $\chi^{2}=4.17,3 \mathrm{df}$; $P=0.24)$.

\section{Discussion}

Our data confirmed that detection of $\mathrm{Ab}$ development after transplantation by $\mathrm{FC}$ is more frequent than by CDC. The percentage of Abpos patients in 143 recipients who received transplants between 1983 and 1993 increased from $6.1 \%$ by CDC to 
$9.8 \%$ by FC. Flow cytometric results were also studied as RCFR, RCFR was determined by classifying the recipients in quartiles according to their percentual change in flow cytometric value from current to posttransplant serum. Thus, $25 \%$ of the patients (quartile 4) showed an increase $>30 \%$ of FC crossmatch positivity. Many of these patients were considered Abneg by classical FC crossmatches. However, their 5 year graft survival is decreased by $40 \%$ and rejection-free survival by $60 \%$ compared with the patients in quartile 1. RCFR therefore is considered a powerful tool in the determination of rejection.

In a number of studies, higher percentages of FC positive patients were found ${ }^{7.9,12}$. The difference with the present results may be partly explained by a different definition of positivity leading to underestimation of the number of positive results. Discrimination between negative and positive results is usually based on the mean of a number of 'negative' human control sera. These negative sera show variability in their reactivity against lymphocytes from different individuals. The cutoff point defined by the mean channel shift plus standard deviations could result in lack of detection of low but significant Ab concentrations ${ }^{9}$. It is obvious that the sensitivity of the technique increases by choosing the negative control value as low as possible. In the study of Utzig et al. ${ }^{8}$, for example, as many as $78 \%$ of the patients with rejections were Abpos with a specificity of $52 \%$. In their study, no human serum was added to the negative control reaction.

To avoid the use of a negative control serum in the definition of the cutoff point, the FC activity of sera before and after transplantation was compared directly. Because both sera were tested with the same target cells, this comparison was considered justified. The FC crossmatch result was expressed as RCFR. A significant effect on graft survival was shown by the log-transformed data $(P=0.0043)$. The height of RCFR was related to both rejection rate and graft survival. About one half of the patients showed an increase in RCFR, whereas the others remained unchanged or showed a decrease. In the RCFR-increased group, a significantly higher rate of rejection and graft failure was noticed compared with the group with no change or decrease.

Each group was divided again, resulting in 4 quartiles, each consisting of about 35 patients. Quartiles with a small increase (quartile $3,<30 \%$ ) or decrease (quartile $2,<10 \%)$ in RCFR differed neither for the incidence of rejection nor graft survival. However, an increase of more than $30 \%$ (quartile 4) detected patients with a high incidence of rejection and lower graft survival, whereas a decrease of more than $10 \%$ (quartile 1) determined a patient group with a low incidence of rejection and high graft survival. The cut-off points in this study are chosen arbitrarily and are merely indicative. For a more precise estimation of the percentages a larger patient population must be studied. 
It is noteworthy that many patients with an increase of $>30 \%$ in fluorescence ratio did not develop antibodies according to the definition given by Scornik? ${ }^{7}$. However their rate of rejection and graft loss was comparable with FC crossmatch positive patients.

As shown previously, 16 patients had a positive current FC crossmatch with a median fluorescence ratio of $4.94^{10}$. Ten of them also had a positive crossmatch with the historical peak serum drawn up to 2 years before transplantation. In this study, 13 out of the 16 current FC-positive patients were demonstrated to become negative after transplantation. They showed good 1-year graft survival $(93 \%)$ and intermediate rejection rate $(46 \%)$. As has been reported by different authors ${ }^{10,13,14}$, a positive FC crossmatch does not necessarily influence the clinical outcome of transplantation. Only three patients remained positive after transplantation. Two were treated for rejection, and one lost his graft within 1 year after transplantation. The number of patients in both groups are small, which makes it difficult to draw definite conclusions. The mechanism by which certain patients turned negative after transplantation is unknown. The occurrence of anti-idiotypic antibodies as described by Suciu-Foca ${ }^{15}$ is a possible although unproven explanation.

Many risk factors are known to influence graft failure or rejection. In this study, both rejection and graft survival were analyzed by Cox regression. Besides wellknown risk factors, such as PRA-grade, HLA-DR match, and gender of recipient, the RCFR contributed significantly to rejection. It has been shown that occurrence of rejection is one of the most important prognostic factors for long term graft survival $^{16,17}$.

As measured by the Cox regression model for selecting risk factors contributing to graft survival (censoring death with function), rejection tended to contribute $(P=0.09)$ if introduced as a single risk factor. When both RCFR and rejection were given as risk factors, RCFR tended to contribute toward graft survival $(P=0.12)$ whereas rejection did not $(P=0.91)$. When RCFR was introduced as the only suspected risk factor, it was shown to be a significant risk factor (overall $P=0.055$; quartile 4 vs. $1 P=0.02$ ). The fact that rejection did not prove to be a significant risk factor cannot be explained by the correlation between rejection and change in fluorescence. Only $40 \%$ of the rejectors belonged to the group with a major increase in fluorescence.

Acute vascular rejection has been shown to influence graft survival in contrast to acute interstitial rejection ${ }^{17}$. Vascular rejection is an Ab-mediated reaction, whereas interstitial rejection is cell-mediated.

A major increase in fluorescence $(>30 \%)$ was noticed in this study in patients without any clinical sign of rejection. These patients also had worse graft survival, 
suggesting subclinical vascular rejection leading to long-term graft loss.

RCFR was the only statistically significant predictor of graft failure in the present investigation. Because this was a retrospective study, it would be interesting to investigate this approach in a prospective study. An increase in median channel $>30 \%$ discriminates at least twice as many patients with high rejection rates and low graft survival than $\mathrm{Ab}$ determination by "standard" FC techniques. Of the patients with functioning graft at 1 year $(n=125)$, those with $>30 \%$ increase had a half life of 4.6 years, in contrast to patients with $>10 \%$ decrease, who had a half life of 15.1 years. The RCFR might not only be indicative of short-term graft loss but also of a higher chance of long-term graft loss. Further prospective studies are necessary to establish the value of this approach in clinical transplantation. 


\section{References}

1. Martin S, Dyer PA, Mallick NP, Gokal R, Johnson RW. Posttransplant anti-donor lymphocytotoxic antibody production in relation to graft outcome. Transplantation 1987; 44: 50-3.

2. Lobo PI, Spencer CE, Stevensson WC, Pruett TL. Evidence demonstrating poor kidney graft survival when acute rejections are associated with IgG donor-specific lymphocytotoxin. Transplantation 1995: 59: 357-60.

3. Talbot D, Givan AL, Shenton BK, Stratton A, Proud G, Taylor RMR. Rapid detection of low leveis of donor specific IgG by flowcytometry with single and dual colour fluorescence in renal transplantation. J Immunol Methods 1988; 112: 279-83.

4. Chapman JR, Deierhoi MH, Carter NP, Ting A, Morris PJ. Analysis of Flow Cytometry and Cytotoxicity Crossmatches in Renal Transplantation. Transplant Proc 1985; 17: 2480-1.

5. Garovoy MR. Flow cytometry crossmatch testing in renal transplantation. Transplant Immunol Lett 1988; 5: 1-2.

6. Talbot D. Givan AL, Shenton BK, Proud G, Taylor RMR. The nature of donorspecific $\operatorname{IgG}$ isotypes identified by flow cytometry in the preoperative crossmatch. Transplant Proc 1988; 20 (suppl 1): 84-5.

7. Scornik J, Salomon D, Lim P. Howard R, Pfaff W. Posttransplant donor antibodies and graft rejection: Evaluation by two-color flow cytometry. Transplantation 1989; 47: $287-90$.

8. Utzig MJ, Blumke M, Wolff-Vorbeck G, Lang H, Kirste G. Flow Cytometry Cross-match: a method for predicting graft rejection. Transplantation 1997; 63: $551-4$.

9. Groth J, Schönemann C, Kaden J, May G. Dynamics of donor-reactive IgG, IgA and $\operatorname{lgM}$ antibodies against $\mathrm{T}$ and $\mathrm{B}$ lymphocytes early after clinical kidney transplantation using flow cytometry. Transplant Immunol 1996; 4: 215-9.

10. Christiaans MHL, Overhof R, ten Haaft A, Nieman F, van Hooff JP, van den BergLoonen PM. No advantage of FC-XM over CDC in immunologically welldocumented renal allograft recipients. Transplantation 1996; 62: 1341-7.

11. van den Berg-Loonen E, Overhof R, Tillemans M, van Hooff H. Allo and auto crossmatches after transplantation. Transpl Int 1992; 5 (Suppl 1): 594-5.

12. Al-Hussein KA, Shenton BK, Bell A, Talbot D, Clark KR, Rigg KM, Forsythe JLR, Proud G. Taylor RMR. Characterization of donor-directed antibody class in the post-transplant period using flow cytometry in renal transplantation. Transpl Int 1994: 7: 182-9.

13. Mahoney RJ, Norman DJ, Colombe BW, Garovoy MR, Leeber DA. Identification of high- and low-risk second kidney grafts. Transplantation 1996; 61: 1349-55.

14. Kerman RH, Van Buren CT, Lewis RM, et al. Improved graft survival for flow cytometry and antihuman-globuline cross-match negative retransplantation recipients. Transplantation 1990; 49: 52-6. 
15. Suciu-Foca N. Reed E, D'Agati VD, Ho E, Cohen DJ, Benvenisty AI, McCabe R, Brensilver JM, King DW, Hardy MA. Soluble HLA antigens, anti-HLA antibodies, and antiidiotypic antibodies in the circulation of renal transplant recipients. Transplantation 1991; 51: 593-601.

16. Basadonna GP, Matas AJ, Gillingham KJ, Payne WD, Dunn DL, Sutherland DER, Gores PF, Gruessner RWG, Najarian JS. Early versus late acute renal allograft rejection: impact on chronic rejection. Transplantation 1993; 55: 993-5.

17. van Saase JLCM, van der Woude FJ, Thorogood J, Hollander AAMJ, van Es LA, Weening J, van Bockel JH, Bruijn JA. The relation between acute vascular and interstitial renal allograft rejection and subsequent chronic rejection. Transplantation 1995; 59 : 1280-5. 


\section{Chapter 7a}

\section{Use of tacrolimus in renal transplantation}

JP van Hooff, MHL Christiaans

Transplantation Proc (in press) 


\section{Introduction and pharmacokinetics}

Tacrolimus is a very potent immunosuppressive agent. It is 10 to 100 times more effective than cyclosporine when tested in vitro. Dosages on a $\mathrm{mg} / \mathrm{kg}$ basis are correspondingly lower than those of cyclosporine. The solid disperse formulation of tacrolimus ensures a rapid oral absorption. This absorption is independent of bile and occurs throughout the gastrointestinal tract, with the duodenum and jejunum being the main sites of absorption. The large degree of interpatient variability of oral bioavailability (range 6\%-43\%) means that monitoring of blood levels is necessary to guide dosing. Whole blood trough level of tacrolimus is a reliable indicator of systemic exposure.

Tracolimus is metabolized by the cytochrome P450 3A4 isoenzyme. Important clinical important interactions exist with drugs that inhibit (e.g., cimetidine, erythromycin) or induce (phenytoin, barbiturates) this cytochrome, resulting in respectively higher and lower systemic exposure of tacrolimus when used concomitantly.

\section{Efficacy}

The efficacy of the drug has been demonstrated in various trials both in Europe and in the USA. One example is the European renal multicenter trial, in which the efficacy of tacrolimus was compared with that of cyclosporine ${ }^{1}$. Patients $(n=448)$ were randomized to receive triple drug therapy consisting of tacrolimus or cyclosporine in combination with low-dose corticosteroids and azathioprine. At 12 months after transplantation, tacrolimus-based immunosuppression was associated with a significant reduction in the frequency of both acute $(25.9 \%$ vs. $45.7 \%)$ and corticosteroid-resistant (11.3\% vs. $21.6 \%)$ rejection. Analysis of biopsies showed that also the percentage of vascular rejection was nearly halved. One-year graft survival rates did not differ significantly between the two treatment groups. The 1-year results of the USA trial with a total of 412 patients were, to a large extent, comparable with those of the European trial ${ }^{2}$. However, in the 3-year analysis the number of graft failures (graft loss excluding death) was significantly lower in the tacrolimus group ${ }^{3}$. These data suggest that the reduced rate and histologic severity of acute rejection in the first year is beginning to yield a difference in graft survival.

Tacrolimus has also been successfully used as a rescue agent for salvage of allografts with refractory rejection under cyclosporine-based immunosuppression. By their very nature, these trials have been non-randomized ${ }^{4}$. The success rates range from $50 \%$ to $100 \%$.

In the USA trial, 34 cyclosporine patients with a proven refractory rejection were 
converted to tacrolimus ${ }^{3}$. The rejection resolved in 26 patients $(76.5 \%)$, while one of the two tacrolimus patients was rescued with cyclosporine-based therapy for refractory rejection. This superior efficacy of tacrolimus has also been demonstrated in retransplants. The incidence of rejection in retransplants on a tacrolimus-based therapy was significantly less than in both primary and retransplant recipients receiving cyclosporines. A summary of the relative efficacy of both compounds is given in Table 7a.1.

Table 7a.1 Efficacy of tacrolimus versus cyclosporine ( + aza +pred).

\begin{tabular}{lcc}
\hline & Tacrolimus & Cyclosporine \\
\hline Acute rejection & & \\
Steroid-resistant rejection & \\
Recurrent rejection & & \\
Vascular rejection & & \\
Treatment failure & & \\
1-year graft survival & & \\
3-year graft survival (USA) & \\
\hline
\end{tabular}

\section{Safety and side effects}

The safety profiles of tacrolimus- and cyclosporine-based regimens are quite comparable ${ }^{1.2}$. There is no difference with regard to patient survival on to incidence of infections, lymphoma, or cancer (Table 7a.2). Serum creatinine and creatinine clearance do not differ, indicating that the nephrotoxic properties of both drugs are the same. Tremor and diarrhea are observed more often with tacrolimus, while hirsutism and gingival hyperplasia are almost exclusively observed during cyclosporine use. A significantly higher incidence of hyperglycemia has been demonstrated in tacrolimus patients than in cyclosporine patients. Risk factors for developing diabetes mellitus are: high levels of tacrolimus, concomitant use of steroids, race, and high body mass index ${ }^{3,6}$. We have found that tacrolimus inhibitisz C-peptide secretion. This secretion improves considerably when levels are decreased. This is consistent with the clinical experience that if diabetes mellitus occurs, dose reduction usually leads to reversal of the diabetes mellitus. We have furthermore observed that a high proportion of renal transplant recipients have a low c-peptide secretion even prior to transplantation. These patients, in particular, are at risk for developing hyperglycemia during the use of tacrolimus ${ }^{6}$.

The cardiovascular risk profile is much more favorable during the use of tacrolimus than of cyclosporine ${ }^{3}$. There is significantly better control of hypertension, and a higher proportion of patients are off antihypertensive drugs. Moreover, lipids (total cholesterol, LDL cholesterol, and triglycerides) are lower in patients on tacrolimus, even though there is significantly less use of statins after 3 
years $(14.4 \% \text { and } 38.7 \% \text { respectively })^{3}$.

The better control of hypertension and more favorable lipid profile may have potentially beneficial effects in the long term because both have been linked to cardiovascular disease and chronic transplant nephropathy. The latter two are the most important complications after successful renal transplantation. A summary of the relative safety and the side effects of tacrolimus and cyclosporine is given in Table 7a.2.

Table 7a.2 Safety and side effects of tacrolimus vs. cyclosporine (+aza + pred).

\begin{tabular}{|c|c|c|c|}
\hline & Tacrolimus & & Cyclosporine \\
\hline Patient survival & & $=$ & \\
\hline Cancer & & $=$ & \\
\hline PTLD & & $=$ & \\
\hline Infection & & $=$ & \\
\hline Nephrotoxicity & & $=$ & \\
\hline Tremor & $\uparrow$ & & \\
\hline Diarrhoea & $\uparrow$ & & \\
\hline Diabetes Mellitus & $\uparrow$ & & \\
\hline Hirsutism & & & $\uparrow$ \\
\hline Gum hyperplasia & & & $\uparrow$ \\
\hline Cardiovascular risk factors & & & $\uparrow$ \\
\hline
\end{tabular}

\section{Optimal combination}

It has been shown that the addition of azathioprine to the combination of tacrolimus and steroids is not uniformly advantageous, although there is a slight reduction in the incidence of rejection. The incidence of steroid-resistant rejection is not influenced by adding azathioprine?

However, the addition of MMF to tacrolimus-based immunosuppression leads to a very low incidence of both acute and steroid-resistant rejection $(9.8 \%-22.9 \%$ and $1.7 \%-4.3 \%$ respectively) ${ }^{8.9}$. A daily dosage of 2 grams of MMF gives a high incidence of gastrointestinal problems. This may be explained by the observation that when MMF and tacrolimus are combined, MPA levels slowly increase over time $^{10}$. Therefore, it seems advisable to use 2 grams of MMF for the initial 2-3 weeks. Thereafter, dosages can be tapered to 1 gram daily ${ }^{10}$.

\section{Summary}

Tacrolimus is a very potent drug for preventing all types of acute rejection after renal transplantation. In particular the decrease in vascular rejection may have 
important long-term implications. This is demonstrated in the 3-year data of the US multicentre trial, where a significant decrease in graft loss was observed in the tacrolimus patients compared to the cyclosporine patients. The safety profiles of both drugs are the same, but there are some major differences in side effects. Hyperglycemia is observed more during tacrolimus administration, but generally the induction of hyperglycemia is a dose-dependent reversible process, that leads generally to diabetes mellitus during high systemic exposure. Moreover, the frequency of hyperglycemia is higher in certain races and in pre-diabetics. Gum hyperplasia and hirsutism are not seen with tacrolimus, and there is a more favorable cardiovascular risk profile during tacrolimus than during use of cyclosporine. 


\section{References}

1. Mayer AD, Dmitrewski J, Squifflet JP, Bessen T, Grabensee B, Klein B, Eigler FW, Heemann U, Pichlmayr R, Behrend M, Vanrenterghem Y, Donck J, van Hooff J, Christiaans M, Morales JM, Andrés A, Johnson RWG, Short C, Buchholz B, Rehmert N, Land W, Schleibner S, Forsythe JLR, Talbot D, Neumayer HH, Hauser I, Ericzon BG, Brattström C, Claesson K, Mühlbacher F, Pohanka E. Multicenter randomized trial comparing tacrolimus (FK506) and cyclosporine in the prevention of renal allograft rejection. Transplantation 1997; 64: 436-43.

2. Pirsch JD, Miller J, Deierhoi MH, Vincenti F, Filo RS. A comparison of tacrolimus (FK506) and cyclosporine for immunosuppression after cadaveric renal transplantation. FK506 Kidney transplant study group. Transplantation 1997; 63: 977-83.

3. Jensik SC for the FK506 Kidney transplant study group. Tacrolimus (FK506) in kidney transplantation: three-year survival results of the US multicenter, randomized, comparative trial. Transplant Proc 1998; 30: 1216-8.

4. Shapiro R. Tacrolimus (FK-506) in kidney transplantation. Transplant. Proc. 1997; 29: 45-7.

5. Pirsch JD, D'Alessandro M, Knechtle SJ, Odorico JS, Becker BN, Kalayoglu M, Sollinger HW. Tacrolimus and retransplantation: equivalent results to primary renal transplantation. Transplantation 1998; 65: S167.

6. van Hooff JP, van Duijnhoven E, Christiaans MHL. Tacrolimus and glucose metabolism. Transplant Proc. 1999 in press.

7. Shapiro R, Jordan ML, Scantlebury VP, Vivas C, Fung JJ, McCauley J, Randhawa P, Demetris AJ, Irish W. Mitchell S, Hakala TR, Simmons RL, Starzl TE. A prospective randomized trial of FK506-based immunosuppression after renal transplantation. Transplantation 1995; 59: 485-90.

8. Mendez R. for the FK506 MMF Dose-ranging Kidney Transplantation Study Group. FK506 and mycophenolate mofetil in renal transplant recipients: six-month results of a multicenter, randomized dose ranging trial. Transplant Proc. 1998; 30: 1287-9.

9. Vanrenterghem Y. Squifflet JP, Forsythe J, Heeman U, Băckman L, Taube D, Morales JM, Ekberg H, van Hooff J, Zanker B, Dietl KH, Talbot D, Hauser I, Tydén G, Claesson K. Co-administration of tacrolimus and mycophenolate mofetil in cadaveric renal transplant recipients. Transplant Proc 1998; 30: 1290-1.

10. Undre NA, van Hooff J, Christiaans M, Vanrenterghem Y, Donck J, Heeman U, Kohnle M, Zanker B, Land W, Morales JM, Andrés A, Schāfer A, Stevenson P. Pharmacokinetics of tacrolimus and mycophenolic acid (MPA) following the administration of a tacrolimus-based regimen in combination with mycophenolate mofetil (MMF) in kidney transplantation. Transplant Proc. 1998; 30: 1299-302. 


\section{Chapter $7 b$}

Pharmacokinetics of tacrolimus and mycophenolic acid (MPA) following the administration of a tacrolimus-based regimen in combination with mycophenolate mofetil (MMF) in kidney transplantation

NA Undre, J van Hooff, M Christiaans, Y Vanrenterghem, J Donck, U Heeman, M Kohnle,

B Zanker, W Land, JM Morales, A Andrés, A Schäfer, P Stevenson 


\section{Introduction}

In clinical trials, mycophenolate mofetil (MMF) in combination with cyclosporin and corticosteroids has been shown to be a potent immunosuppressive agent in cadaveric renal transplantation ${ }^{1 \cdot 3}$.

After absorption, MMF is hydrolysed to its pharmacologically active form mycophenolic acid (MPA)4. A large portion of MPA is further metabolised to its glucuronide (MPAG), which is inactive, prior to its elimination ${ }^{5}$.

Very little is known about the pharmacokinetics of MMF in organ transplant recipients receiving tacrolimus based immunosuppressive regimen. The present report describes the pharmacokinetics of tacrolimus and MMF in renal allograft recipients receiving both agents.

The objectives of this study were to obtain information on the effect of concomitant administration of MMF on the pharmacokinetics of tacrolimus and to characterise the pharmacokinetics of MPA after the first dose and at steady state when administered in combination with tacrolimus.

\section{Methods}

\section{Subjects}

The pharmacokinetics were evaluated in a subset of adult patients who were recruited into a multi-centre, randomised, parallel-group, dose-finding study and received a single-organ cadaveric renal transplant.

A total 63 patients ( 42 males, 21 females), with a mean age of 48.3 years (range $22-75$ years) and a mean weight of $69.2 \mathrm{~kg}$ (range $37-108 \mathrm{~kg}$ ), were randomised to one of the following three treatment groups:

1. Tacrolimus and corticosteroids - without MMF $(\mathrm{n}=23)$,

2. Tacrolimus, corticosteroids, and $1 \mathrm{~g} /$ day MMF $(n=19)$,

3. Tacrolimus, corticosteroids, and $2 \mathrm{~g}$ /day MMF $(n=19)$,

Two patients did not provide adequate pharmacokinetic profiles.

Oral tacrolimus (initial daily dose of $0.2 \mathrm{mg} / \mathrm{kg}$ adjusted for target blood levels below $15 \mathrm{ng} / \mathrm{mL}$ ) and MMF were administered in 2 divided doses per day. 


\section{Pharmacokinetic profiles}

Whole blood samples were collected on day 3 (first dose of MMF), and on two occasions at steady state, approximately 2 weeks and 3 months post-transplantation. Samples were collected just before administration ( 0 hour) and at $0.5,1,2,3,4$, $6,8,9,10,11$, and 12 hours after administration.

\section{Assay methods}

Concentrations of tacrolimus in blood were determined by an HPLC-MS/MS assay ${ }^{6}$. MPA and MPAG levels were determined by the HPLC method of Tsina et $\mathrm{al}^{7}$. Reference standards for the MPA and MPAG assays were a gift from Roche Bioscience, Palo Alto, USA.

\section{Pharmacokinetic analysis}

Model-independent methods were used to estimate the pharmacokinetic parameters with the computer program TOPFIT version 2.0.

As tacrolimus therapy is based on adjusting the dose for the blood concentrations within target range, any effect of MMF on the pharmacokinetics of tacrolimus was evaluated by comparing the dose normalised (dose-normalised to $0.1 \mathrm{mg} / \mathrm{kg}$ ) area under the concentration-time profile (AUC) between treatment groups.

\section{Results}

\section{Tacrolimus pharmacokinetics}

The oral absorption profiles of tacrolimus collected on three occasions from each of the three treatment groups are illustrated in Figure $7 \mathrm{~b} .1$. The median times to peak concentration which ranged from 1.1 to 2.3 hours indicated that tacrolimus was rapidly absorbed. The effect of concomitant administration of MMF on the pharmacokinetics of tacrolimus was evaluated by comparing the dose-normalised AUC of the three treatment groups. A difference in this parameter among the three treatment groups was not discernible (see Figure 7b.2). 

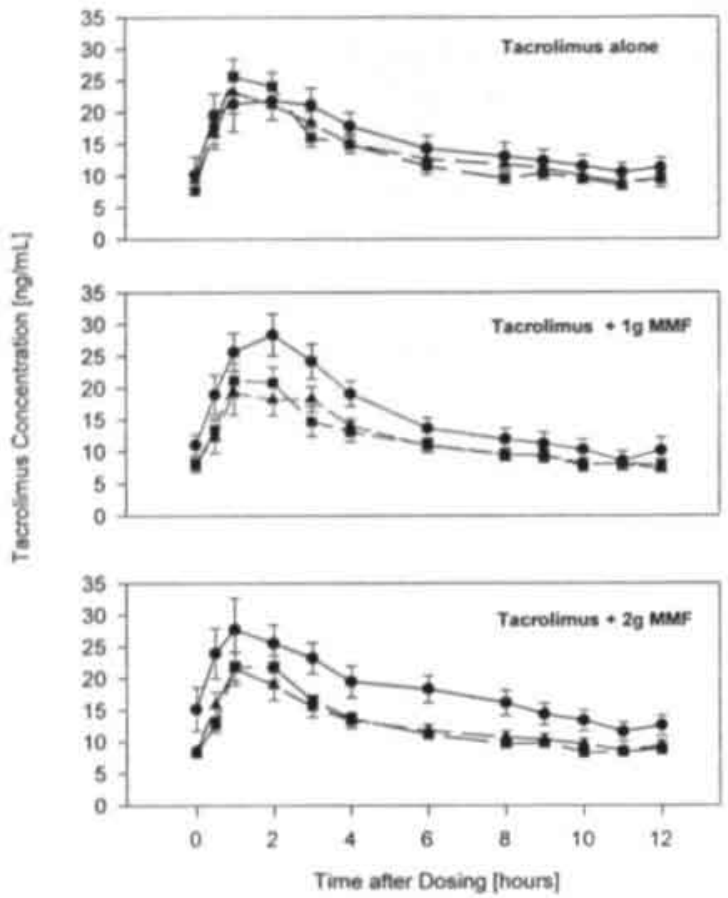

Figure 7b.1 Mean \pm SEM oral absorption profiles of tacrolimus on day $3(\bullet)$, week $3(\mathbf{E})$. and month 3 (A) posttransplant.

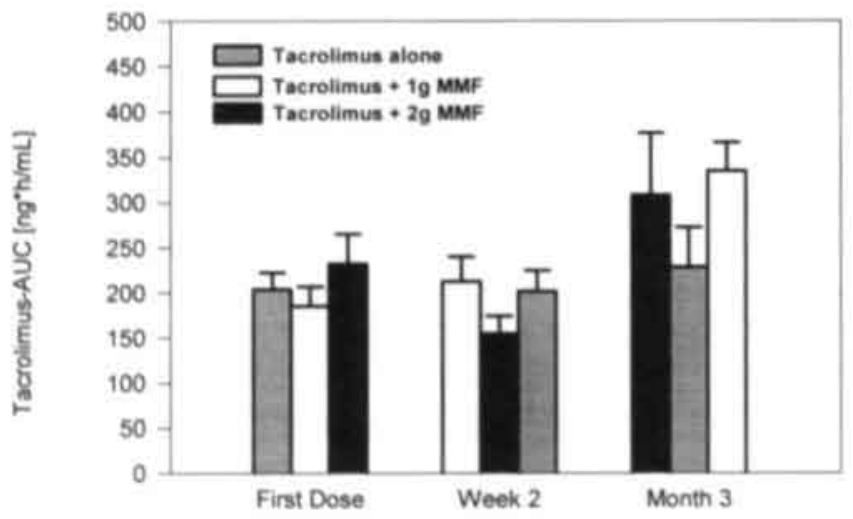

Figure 7b.2 Comparison of mean \pm SEM dose-normalised AUC of tacrolimus following the oral administration of tacrolimus. 


\section{MPA pharmacokinetics}

The blood concentration-time profiles of MPA following the 1-g and the 2-g doses of MMF on three occasions are illustrated in Figure $7 \mathrm{~b} .3$. There was a considerable overlap in the individual AUC values in the two dosing groups (see Figure 7b.4). The mean AUC values for MPA in the 1-g and 2-g groups at week 2 were approximately $25 \mu \mathrm{g} \cdot \mathrm{h} / \mathrm{mL}$ and $40 \mu \mathrm{g} \cdot \mathrm{h} / \mathrm{mL}$, respectively. Corresponding AUC values at month 3 were approximately $20-30 \%$ higher (see Figure $7 \mathrm{~b} .5$ ).
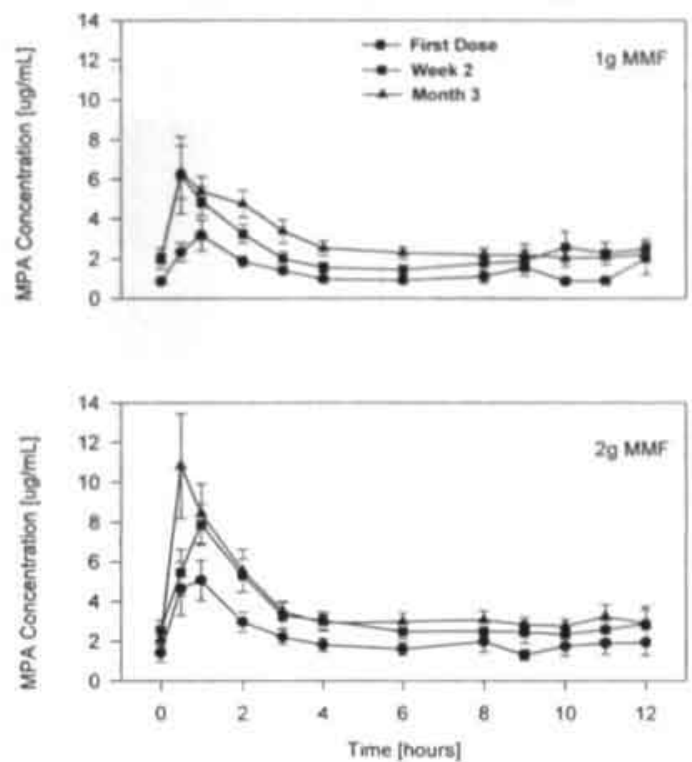

Figure $7 \mathrm{~b} .3$ Mean \pm SEM concentration-time profile of MPA following oral administration of $1-\mathrm{g}$ or 2-g MMF in combination with tacrolimus.

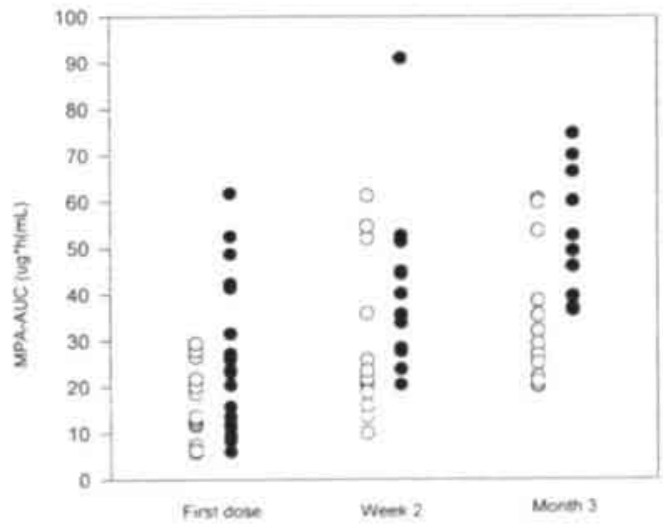

Figure 7b.4 Individual MPA AUC values following oral administration of $1 \mathrm{gg}$ or $2 \mathrm{~g} \mathrm{MMF}$ in combination with tacrolimus. 

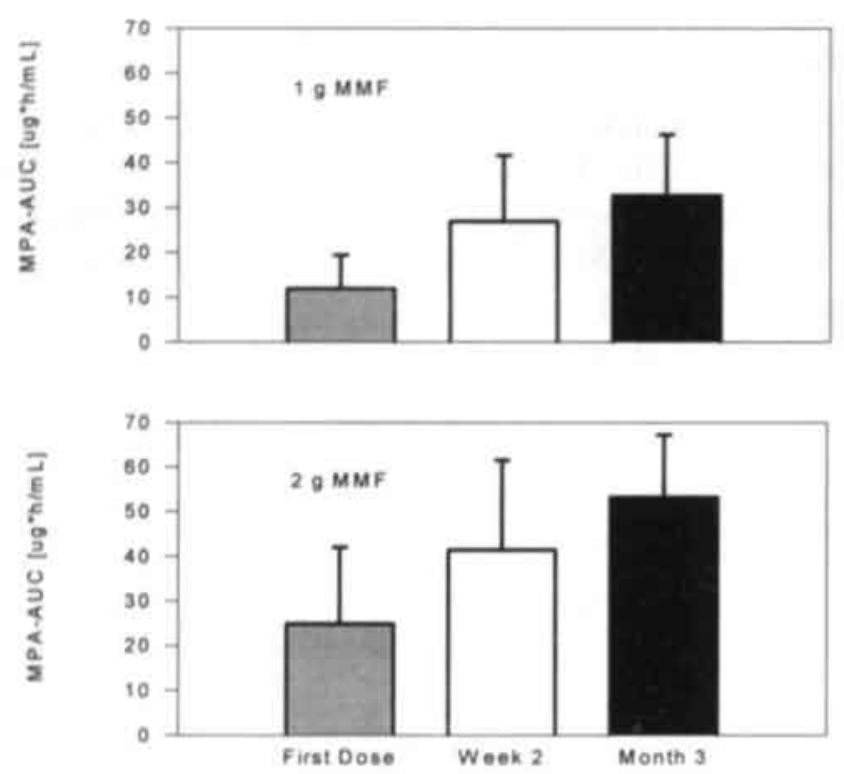

Figure 7b.5 Mean \pm SD MPA AUC values following oral administration of $1-\mathrm{g}$ or $2-\mathrm{g}$ MMF in combination with tacrolimus.

\section{Conclusions}

An effect on the absorption kinetics of tacrolimus, in terms of the rate of absorption or extent of systemic availability, was not discernible with concomitant administration of MMF. This suggests that, in order to achieve tacrolimus blood levels within the recommended target range, no adjustment in tacrolimus dosing is necessary when MMF is administered concomitantly.

There was a considerable overlap in the individual AUC values of MPA at all time points between the 1-g and the 2-g MMF groups, suggesting that the 1-g MMF dosage may provide adequate systemic exposure when given in combination with tacrolimus. The increase in AUC at month 3 suggests that the dose of MMF may need to be decreased with time post-transplant in order to maintain stable systemic exposure to MPA. 


\section{References}

1. European Mycophenolate Mofetil Cooperative Study Group. Placebo-controlled study of mycophenolate mofetil combined with cyclosporine and corticosteroids for prevention of acute rejection. Lancet 1995; 345: 1321-5.

2. The Tricontinential Mycophenolate Mofetil Renal Transplantation Study Group. A blinded, randomized clinical trial of mycophenolate mofetil for the prevention of acute rejection in cadaveric renal transplantation. Transplantation 1996; 61: 1029-37.

3. Sollinger HW for the U.S. Renal Transplant Mycophenolate Mofetil Study Group. Mycophenolate mofetil for the prevention of acute rejection in primary cadaveric renal allograft recipients. Transplantation 1995; 60: 225-32.

4. Lee WA, Gu L, Miksztal A, Chu N, Leung K, Nelson PH. Bioavailability improvement of mycophenolic acid through amino ester derivatization. Pharm Res 1990; 7: 161-6.

5. Sweeney MJ, Hoffman DH, Esterman MA. Metabolism and biochemistry of mycophenolic acid. Cancer Res 1972; 32: 1803-9.

6. Hill HM, Clarke SD, Bentley L, Noctor TAG, Iwasaki K, Shiraga T, Hata T, Undre N. A high sensitivity assay for tacrolimus (FK506) in human blood. Presented at the 1997 AAPS annual meeting, Boston 2-6 November 1997.

7. Tsina I, Chu F, Hama K, Kaloostian M, Tam YL, Tarnowski T, Wong B. Manual and automated (robotic) high-performance liquid chromatography methods for the determination of mycophenolic acid and its glucuronide conjugate in human plasma. J Chromatography B 1996; 675: 119-29. 


\section{Chapter 7c}

\section{Dosing and management guidelines for tacrolimus in renal transplant patients}

JP van Hooff, JMM Boots, EM van Duijnhoven, MHL Christiaans

Transplantation Proc (in press) 


\section{Introduction}

When tested in in vitro models of immunosuppression, tacrolimus is 10 to 100 times more potent than cyclosporine. Thus, corresponding doses on a $\mathrm{mg} / \mathrm{kg}$ basis are much lower than those of cyclosporine. Tacrolimus is metabolized by the cytochrome P450 3A4 isoenzyme, which is present in the upper gastrointestinal tract and the liver'. Substances known to inhibit this isoenzyme (e.g., cimetidine, erythromycin) may therefore decrease the metabolism of tacrolimus (increase blood levels) and, conversely, drugs known to induce this isoenzyme (e.g., phenytoin, barbiturates) may increase the metabolism of tacrolimus (decrease blood levels).

Hepatic impairment decreases the clearance of tacrolimus and thus lower doses may be required in these patients. However, renal function does not influence drug clearance. After transplantation clearance decreases with time, leading to a reduction in the dose required'.

\section{Relationship between whole blood levels, efficacy and toxicity}

In an attempt to define an optimal dosing regimen, a prospective dose-finding study was conducted in renal transplant patients in the USA. A significant linear trend towards increasing rates of toxicity with increasing trough concentrations of tacrolimus was observed. Conversely, episodes of acute rejection were associated with lower trough concentrations. Results from this study indicated that trough levels between $5 \mathrm{ng} / \mathrm{mL}$ and $25 \mathrm{ng} / \mathrm{mL}$ should be targeted in an attempt to optimize the risk/benefit ratio ${ }^{2}$.

These findings were confirmed and extended by an analysis of a European multicenter trial. In this study, the risk of acute rejection was higher in patients with low systemic exposure during the early posttransplant period. It was concluded that to reduce the risk of rejection, a minimum trough concentration of $10 \mathrm{ng} / \mathrm{mL}$ should be achieved by day 2 to 3 posttransplant ${ }^{3}$. Conversely, the risk of toxicity (tremor, diabetes, nephrotoxicity) was significantly higher with prolonged exposure to blood levels $>20 \mathrm{ng} / \mathrm{mL}^{\prime}$.

\section{Dosing and monitoring}

The solid dispersion formulation of tacrolimus is rapidly absorbed after oral administration and the mean bioavailability is approximately $20 \%$ (range $6 \%$ to $43 \%)$ in renal transplant patients'. This degree of variability means that the dosage needs to be individualized using tacrolimus whole blood trough concentration 
monitoring as a guide. There is a highly significant correlation between area under the concentration-time curve and trough levels of tacrolimus, indicating that trough levels are reliable indicators of systemic exposure'.

Generally, in renal transplant patients, tacrolimus is initiated orally at 0.2 to 0.3 $\mathrm{mg} / \mathrm{kg}$ body weight in two divided doses. It has been recommended that the first oral dose of tacrolimus is given within 24 hours of surgery. If one follows this advice, almost $30 \%$ of patients have a trough level below $10 \mathrm{ng} / \mathrm{mL}$ on day 7 and are therefore at risk of rejection ${ }^{4}$. If the first dose is administered before surgery, less than $5 \%$ of patients have levels below $10 \mathrm{ng} / \mathrm{mL}$ on day $7^{4}$.

During the early posttransplant period, regular monitoring of trough levels is recommended. Due to the long half-life of tacrolimus (range 12 to 16 hours), it is not necessary to measure blood levels on a daily basis. In general, tacrolimus doses should be changed in $20 \%$ to $40 \%$ steps with no more than two dose changes per week. Therefore, blood level monitoring should be undertaken 2 to 3 times per week. Additional monitoring might be necessary based on clinical indications.

During maintenance therapy, less frequent blood level monitoring is required. Trough levels should be monitored during regular check-up visits. Additional monitoring should be undertaken after dose adjustment, after switching from another immunosuppressive regimen, or following administration of drugs that could potentially lead to interactions.

\section{Therapeutic conduct at the Maastricht Transplantation Centre}

The policy at the Maastricht Transplantation Centre is to avoid underdosing in the early postoperative period. Underdosing leads to an increased risk of rejection, while toxic effects of overdosing are reversible when doses are tapered. Generally, one or two doses of tacrolimus $(0.1 \mathrm{mg} / \mathrm{kg})$ are administered orally before transplantation. Patients are started on $0.3 \mathrm{mg} / \mathrm{kg} /$ day within 24 hours of surgery. The trough level of tacrolimus is measured 2 days after surgery and is generally between $15 \mathrm{ng} / \mathrm{mL}$ to $20 \mathrm{ng} / \mathrm{mL}$. In exceptional cases where the level is below $5 \mathrm{ng} / \mathrm{mL}$ the dose is doubled. In these circumstances, a pharmacokinetic profile is performed to investigate whether the low level is due to poor absorption or rapid metabolism of the drug. If the level is between $5 \mathrm{ng} / \mathrm{mL}$ and $10 \mathrm{ng} / \mathrm{mL}$ the dose is increased by $20 \%$ to $40 \%$ and if it is between $10 \mathrm{ng} / \mathrm{mL}$ and $15 \mathrm{ng} / \mathrm{mL}$ the dose is increased by $10 \%$ to $20 \%$. When the level is between $20 \mathrm{ng} / \mathrm{mL}$ and $30 \mathrm{ng} / \mathrm{mL}$ the dose is decreased by $20 \%$ to $40 \%$. If the level is between $30 \mathrm{ng} / \mathrm{mL}$ and $40 \mathrm{ng} / \mathrm{mL}$ the dose is decreased by $50 \%$. In rare cases when levels are above $40 \mathrm{ng} / \mathrm{mL}$ with concomitant toxicity, the evening dose is omitted and the dose on the following day is reduced by $50 \%$ or more (Table $7 \mathrm{c} .1$ ). The trough levels of tacrolimus are between $15 \mathrm{ng} / \mathrm{mL}$ and $20 \mathrm{ng} / \mathrm{mL}$ during the first 2 weeks after transplantation, 
$10 \mathrm{ng} / \mathrm{mL}$ to $15 \mathrm{ng} / \mathrm{mL}$ in weeks 3 and 4 , and between $5 \mathrm{ng} / \mathrm{mL}$ and $10 \mathrm{ng} / \mathrm{mL}$ thereafter during the first year. After the first year, the target trough levels are between $5 \mathrm{ng} / \mathrm{mL}$ and $7 \mathrm{ng} / \mathrm{mL}$.

Corticosteroid pulse therapy is used if an acute rejection episode occurs. In these cases, the dose of tacrolimus is increased if trough levels are below $15 \mathrm{ng} / \mathrm{mL}$.

Table 7c.1 Therapeutic conduct at the Maastricht Transplantation Centre.

\begin{tabular}{ll}
\hline Preoperative & Tacrolimus $0.1 \mathrm{mg} / \mathrm{kg}$ once or twice daily \\
Postoperative (start <24 hours after surgery) & $0.3 \mathrm{mg} / \mathrm{kg} / \mathrm{day}$ orally \\
Tacrolimus trough level at day $2-3$ & $15-20 \mathrm{ng} / \mathrm{mL}$ (target) \\
Adjustment of dose according to tacrolimus trough level \\
$<5 \mathrm{ng} / \mathrm{mL}$ & Increase dose by $100 \%$ after pharmacokinetic profile \\
$5-10 \mathrm{ng} / \mathrm{mL}$ & Increase dose by $20 \%-40 \%$ \\
$10-15 \mathrm{ng} / \mathrm{mL}$ & Increase dose by $10 \%-20 \%$ \\
$15-20 \mathrm{ng} / \mathrm{mL}$ & Dose unchanged \\
$20-30 \mathrm{ng} / \mathrm{mL}$ & Decrease dose by $20 \%-40 \%$ \\
$30-40 \mathrm{ng} / \mathrm{mL}$ & Decrease dose by $50 \%$ \\
$>40 \mathrm{ng} / \mathrm{mL}$ & Omit one dose and decrease next dose by $50 \%$ or more \\
\hline
\end{tabular}

\section{Influence of food and diabetic state}

There is a large interindividual variability in oral bioavailability of tacrolimus in diabetic patients $s^{3}$. Therefore, it is logical to perform routine pretransplant pharmacokinetic profiles to detect patients with low systemic exposure, as has been suggested for cyclosporine ${ }^{6}$.

After renal transplantation in nondiabetic patients, the mean difference in systemic exposure to tacrolimus in the fasted and nonfasted state is only $2 \%$ at 3 weeks? This may be the best time to start taking tacrolimus with food. Stringent monitoring of trough levels is necessary because there is a higher interpatient variability in bioavailability in the nonfasting state. In diabetic patients, careful dose adjustment may be required because in some individuals a clinically important decrease in absorption of tacrolimus occurs when it is taken with food ${ }^{8}$.

Stable nondiabetic renal transplant patients who have been taking tacrolimus on an empty stomach for 1 year after transplantation and would like to switch to taking the medication with food, should be on a stable dose with trough levels between 8 and $10 \mathrm{ng} / \mathrm{mL}$. When starting to take tacrolimus with food, the dose should initially be increased by $25 \%$ to avoid low levels. Generally, thereafter, doses are tapered on an individual basis to achieve preconversion trough levels? 


\section{Combination with other immunosuppressants}

In clinical practice tacrolimus is combined with other agents such as corticosteroids and azathioprine or mycophenolate mofetil (MMF). If trough levels of tacrolimus directly after surgery are between $15 \mathrm{ng} / \mathrm{mL}$ and $20 \mathrm{ng} / \mathrm{mL}$, the chance of acute rejection is low and concomitant induction therapy with poly- or monoclonal antibodies is not necessary.

The efficacy of the different combinations varies considerably (Table 7c.2). When tacrolimus is combined with corticosteroids, the acute rejection rate is approximately $27 \%$ and corticosteroid-resistant rejection ranges from $6 \%$ to $11 \%$. 10 . In two large multicenter studies, it was found that the addition of azathioprine to tacrolimus/corticosteroid-based immunosuppressive therapy did not convey increased efficacy ${ }^{9,10}$. The combination of tacrolimus, corticosteroids and $\mathrm{MMF}^{11,12}$ leads to a further reduction in the rejection rate to approximately $16 \%$ and $3 \%$ for corticosteroid-sensitive and corticosteroid-resistant rejection, respectively. With the latter combination there is only a minor increase in morbidity compared with the other combinations.

There are clinically important pharmacologic interactions between tacrolimus and corticosteroids and between tacrolimus and MMF. Corticosteroids increase the metabolism of tacrolimus by induction of the cytochrome P450 3A4 isoenzyme leading to decreased blood levels of tacrolimus ${ }^{13}$. Concomitant administration of tacrolimus and MMF leads to a steadily increasing level of the pharmacologically active form of MMF, mycophenolic acid. After 2 weeks of concomitant administration of MMF $1 \mathrm{~g}$ twice daily and tacrolimus, the dose of MMF needs to be reduced to avoid side effects; thereafter, MMF $1 \mathrm{~g} /$ day may provide adequate systemic exposure ${ }^{14}$.

Table 7c.2 Efficacy of tacrolimus combined with other immunosuppressants.

\begin{tabular}{ccc}
\hline & $\begin{array}{c}\text { Acute } \\
\text { rejection (\%) }\end{array}$ & $\begin{array}{c}\text { Corticosteroid-resistant } \\
\text { acute rejection (\%) }\end{array}$ \\
\hline $\begin{array}{c}\text { Tacrolimus + prednisone } \\
\text { Segoloni et al. }\end{array}$ & 28 & 6 \\
Chang et al. ${ }^{10}$ & 28 & 11 \\
Tacrolimus + prednisone + azathioprine & 29 & 5 \\
Segoloni et al. & 26 & 12 \\
Chang et al. & & \\
Tacrolimus + prednisone + MMF 2 g/day & 23 & 10 \\
Vanrenterghem et al. & 10 & 2 \\
Mendez et al. & & \\
\hline
\end{tabular}




\section{Withdrawal of concomitant therapy}

\section{Corticosteroids}

The Pittsburgh group was the first to show that a large proportion of patients can be weaned off corticosteroids at 1 or 2 years after transplantation ${ }^{15}$. Recently, it was demonstrated that early weaning is also possible ${ }^{16}$. In Maastricht, corticosteroids were stopped at a median of 449 days posttransplant in 57 patients. No acute rejection episodes occurred during the median follow-up of 331 days. Of these patients, $44(77 \%)$ are now on tacrolimus monotherapy. Excluded from the late weaning protocol were three patients with new-onset proteinuria and five highrisk patients.

\section{Azathioprine}

There are no data available from randomized trials regarding withdrawal of azathioprine, although some anecdotal data are available. In a European multicenter study, where patients received tacrolimus, azathioprine and $5 \mathrm{mg}$ prednisone, $77 \%$ of patients were successfully withdrawn from azathioprine after 3 months ${ }^{17}$.

\section{MMF}

Although controlled data are lacking, there is circumstantial evidence that MMF can be withdrawn. In a European trial comparing three doses of MMF in combination with corticosteroids and tacrolimus, only one episode of rejection (which was reversible) occurred in 30 patients in whom MMF had been withdrawn because of adverse events. In this trial, patients were followed-up for only 6 months ${ }^{11}$. Therefore, an analysis of the long-term effect was conducted in a subset of patients who underwent transplantation at 3 closely co-operating centres (Brussels, Leuven and Maastricht). After 2 years, only $12.5 \%$ of patients were still using MMF and it was successfully withdrawn in the remainder ${ }^{18}$.

More data will soon become available because the effect of withdrawing MMF and corticosteroids is the subject of an ongoing prospective, randomized, multicenter, European trial.

\section{Optimal combinations}

Cadaveric renal allografts are transplanted under the auspices of organ exchange organizations to improve HLA matching. In particular, DR identity between donor and recipient is feasible for a high proportion of patients because of the restricted polymorphism of the DR system. There is a strong influence of HLA-DR matching 
on the incidence of acute rejection episodes in patients using tacrolimus, prednisolone and azathioprine. In DR-identical recipients receiving this combination the rejection-free rate was more than $80 \%{ }^{19}$.

On the basis of the aforementioned findings, a tentative scheme of tailor-made immunosuppression for Caucasian renal transplant recipients has been proposed (Table 7c.3).

Low-risk recipients (first graft, panel reactive antibody $<20 \%$, DR-identical) can be initially treated with tacrolimus and low-dose corticosteroids. In cases of corticosteroid-resistant rejection, MMF is added and patients are maintained on a triple-drug regimen. In the remaining patients, corticosteroids can be weaned, especially in those patients who have not been treated for rejection.

All other recipients are initially treated with tacrolimus, low-dose corticosteroids and MMF, administered at a dose of $1 \mathrm{~g}$ twice daily in the first 2 weeks and $0.5 \mathrm{~g}$ twice daily thereafter. Hyperimmunized patients and patients with corticosteroidresistant rejection are maintained on a triple-drug regimen. Recipients not requiring rejection therapy can be weaned off corticosteroids after 3 months and MMF can be withdrawn after 6-12 months.

Table 7c.3 Proposal for tailor-made immunosuppression for Caucasian renal transplant patients.

\begin{tabular}{lccc}
\hline & Tacrolimus & Corticosteroid & MMF $^{\mathbf{b}}$ \\
\hline Low risk patients & + & + & \\
All other patients & + & + & + \\
\hline
\end{tabular}

- Corticosteroids: if rejection occurs, maintain on $5 \mathrm{mg}$, otherwise aim to withdraw by $2-3$ months.

MMF: $1 \mathrm{~g}$ twice daily week $0-2 ; 0.5 \mathrm{~g}$ twice daily week 3 onwards. Maintain treatment in hyperimmunized patients and patients with corticosteroid-resistant rejection and/or vascular rejection; withdraw in all other patients after month 6. 


\section{References}

1. Undre NA, Stevenson P, Schäfer A. Pharmacokinetics of tacrolimus: clinically relevant aspects. Transplant Proc 1999. In press.

2. Laskow DA, Vincenti F, Neylan J, Mendez R, Matas A. Phase II FK506 multicenter concentration control study: one-year follow-up. Transplant Proc 1995; 27: 809-11.

3. Undre NA, van Hooff JP, Christiaans M, Vanrenthergem Y, Donck J, Heeman U, Kohnle M. Zanker B, Land W, Morales JM, Andrés A, Schäfer A, Stevenson P. Low systemic exposure to tacrolimus correlates with acute rejection. Transplant Proc 1999; 31: 296-8.

4. Boots JMM, Christiaans $M$, van Hooff JP: In preparation.

5. van Duijnhoven E, Christiaans M, Schafer A, Undre N, van Hooff J. Tacrolimus dosing requirements in diabetic and nondiabetic patients calculated from pretransplantation data. Transplant Proc 1998; 30: 1266-7.

6. Kahan BD, Ried M, Newburger J. Pharmacokinetics of cyclosporine in human renal transplantation. Transplant Proc 1983; 15: 446-53.

7. Christiaans M, van Duijnhoven E, Beysens T, Undre N, Schäfer A, van Hooff J. Effect of breakfast on the oral bioavailability of tacrolimus and changes in pharmacokinetics at different times posttransplant in renal transplant recipients. Transplant Proc 1998; 30: 1271-3.

8. van Duijnhoven E, Christiaans M, Undre N, Stevenson P, van Hooff J. The effect of breakfast on the oral bioavailability of tacrolimus in diabetic and nondiabetic patients before transplantation. Transplant Proc 1998; 30: 1268-70.

9. Segoloni GP, for the Italian and Spanish Tacrolimus Study Group. Tacrolimus is highly effective in both dual and triple therapy regimens following renal transplantation. 9th Congress of the European Society for Organ Transplantation. Abstract 115, 1999

10. Chang RWS, Palmer A, Kwan J. Nicholson M, Kashi S, Fernando O, Perner F. Neild GH. European multicenter study comparing a dual vs. triple low-dose tacrolimus regimen in renal transplantation. 9th Congress of the European Society for Organ Transplantation. Abstract 1149, 1999.

11. Vanrenterghem Y. Squifflet JP. Forsythe J, Heeman U, Bäckman L, Taube D. Morales JM, Ekberg H, van Hooff J, Zanker B, Dietl KH, Talbot D, Hauser I, Tydèn G, Claesson K. Mühlbacher F. Co-administration of tacrolimus and mycophenolate mofetil in cadaveric renal transplant recipients. Transplant Proc 1998; 30: 1290-1.

12. Mendez R, for the FK 506 MMF Dose-Ranging Kidney Transplant Study Group. FK506 and mycophenolate mofetil in renal transplant recipients: Six-month results of a multicenter, randomized dose ranging trial. Transplant Proc 1998; 30: 1287-9.

13. Undre NA, Schäfer A, and the European Tacrolimus Multicentre Renal Study Group: Factors affecting the pharmacokinetics of tacrolimus in the first year after renal transplantation. Transplant Proc 1998; 30: 1261-3. 
14. Undre NA, van Hooff JP, Christiaans M, Vanrenthergem Y, Donck J, Heeman U, Kohnle M, Zanker B, Land W, Morales JM, Andrés A, Schäfer A, Stevenson P. Pharmacokinetics of FK506 and mycophenolic acid after the administration of a FK506-based regimen in combination with mycophenolate mofetil in kidney transplantation. Transplant Proc. 1998; 30: 1299-302.

15. Shapiro R, Jordan ML, Scantlebury VP, Vivas C, Fung J, McCauley J, Randhawa P, Demetris AJ, Irish W, Jain A, et al. A prospective, randomized trial of FK506/prednisone vs. FK/azathioprine/prednisone in renal transplant patients. Transplant Proc 1995; 27: 814-7.

16. Woodle ES, Buell J, Siegel C, Kulkarni S, Kopelan A, Grewal HP. Corticosteroid withdrawal under tacrolimus primary and rescue therapy in renal transplantation: The Chicago experience. Transplant Proc 1999 (in press)

17. Mayer AD, Dmitrewski J, Squifflet JP, Bessen T, Grabensee B, Klein B, Eigler FW, Heemann U, Pichlmayr R, Behrend M, Vanrenterghem Y, Donck J, van Hooff J, Christiaans M, Morales JM. Andrés A, Johnson RWG, Short C, Buchholz B, Rehmert N, Land W, Schleibner S, Forsythe JR, Talbot D, Neumayer HH, Hauser I, Ericzon BG, Brattstrōm C, Claesson K, Mühlbacher F, Pohanka E. Multicenter randomized trial comparing tacrolimus (FK506) and cyclosporine in the prevention of renal allograft rejection. Transplantation 1997; 64: 436-43.

18 Squifflet JP, van Hooff JP, Vanrenterghem Y. The Benelux experience with the combination of tacrolimus and mycophenolate mofetil. Transplant Proc 1999 (in press).

19. Richards E, Schleibner S, Talbot D, and the European Tacrolimus Multicentre Renal Study Group. An exploratory analysis of prognostic factors for patient outcome during the first year following renal transplantation. Transplant Proc 1998; 30: $1386-8$. 


\section{Chapter 8}

Multivariate analysis of factors related to immediate and longterm clinical outcome in cadaveric renal transplantation: a single-center study

MHL Christiaans, F Nieman, G Kootstra, EM van den Berg-Loonen, JP van Hooff 


\section{Introduction}

Renal transplantation is a first choice therapy for most patients who need renal replacement therapy ${ }^{1}$. Currently, more patients are alive with a functioning graft than are on hemodialysis (HD) or peritoneal dialysis (CAPD) $)^{2}$. Most of the transplants have been performed with cadaveric grafts. The results of cadaveric renal transplantation have improved in recent decades. This has been attributed to the introduction of cyclosporin, to better screening for HLA antibodies, to HLA matching and crossmatching, and to an improvement in patient management after transplantation, especially by prevention and treatment of infections and cardiovascular diseases.

However, because of the increase in the number of patients on the waiting list for kidney transplantation, the donor criteria have been changed to also include nonoptimal donors such as non-heart-beating donors (NHB) and older donors. This may influence immediate post-transplant clinical outcome parameters such as delayed graft function (DGF), acute rejection, and duration of hospitalizsation, as well as long-term parameters such as patient (PS) and graft survival (GS) and quality of function of the graft.

Registry studies have been performed; however, these have involved heterogeneous populations with different pretransplant selections, transplant procedures, and posttransplant management. Furthermore, the quality of the data in these registries is insufficient to analyze such outcome parameters as duration of hospitalization, quality of renal function, and duration of DGF in detail.

This study was designed to investigate the influence of histocompatibility and of donor and patient parameters on these outcome measures in a cohort of consecutive, cadaveric renal transplants in the University Hospital Maastricht. During the period of study, the immunosuppressive regimen as well as the tissue typing procedure were uniform, thereby making it possible to analyze the impact of the abovementioned parameters on clinical outcome parameters in a homogeneous group of patients.

\section{Materials and methods}

\section{Patients}

A total of 299 patients received 346 consecutive cadaveric renal transplants in our center from September 1983 to September 1995. Thirty-nine recipients received two grafts and four patients three grafts. All recipients were treated with a cyclosporine ( $\mathrm{CsA})$-based immunosuppressive regimen after transplantation. Of the 
recipients, 224 were male $(64.7 \%)$ and 122 were female $(35.3 \%)$. The mean age of the recipients was 47.0 years (SD 12.79 years). Of the recipients, 265 had received a first transplant $(76.6 \%), 64$ a second $(18.5 \%), 13$ a third $(3.8 \%)$, and 4 a fourth $(1.2 \%)$. At the time of transplantation, one investigator assigned a ranking score to each patient indicating his or her suitability as a candidate for kidney transplantation (general evaluation) based on medical status (e.g., diabetes mellitus, cardiovascular diseases) and previous transplant history (immunization grade, transplant number, reason for failure of previous graft). A total of 174 patients $(50.3 \%)$ scored 'good', $129(37.6 \%)$ 'moderate', and 24 (6.9\%) 'poor'; for 18 patients $(5.2 \%)$ the score was missing and they were omitted from analyzes in which the item was included as a significant parameter. Characteristics of our study population are shown in Table 8.1.

\section{Immunosuppression}

The immunosuppressive regimen consisted of CsA and low-dose prednisolone (PRED; $10 \mathrm{mg} /$ day) for recipients of first grafts. The CsA was started intravenously before surgery (continuous infusion; $4 \mathrm{mg} / \mathrm{kg}$ every $24 \mathrm{~h}$ ) and then switched to oral administration ( $4 \mathrm{mg} / \mathrm{kg}$ b.i.d.) at day 2 . The PRED dose was diminished to $7.5 \mathrm{mg} /$ day at month 1 and to $5 \mathrm{mg} /$ day at month 3 . For recipients without rejection, the PRED dose was further tapered to $0 \mathrm{mg}$ in the months that followed. For highly immunized recipients (panel reactive antibodies (PRA) current-serum $>85 \%$ ) and retransplant recipients due to immunological failure of a previous graft, azathioprine (AZA, $\pm 1 \mathrm{mg} / \mathrm{kg}$ body weight) was added to the above-described regimen and the dose of PRED was tapered to $5 \mathrm{mg}$. The CsA level was determined in whole blood by high-performance liquid chromatography (HPLC) or by monoclonal radioimmunoassay. The trough CsA level for CsA+PRED recipients was $0.15-0.20 \mathrm{mg} / \mathrm{L}$ in the first 3 months after transplantation and $0.10-0.15 \mathrm{mg} / \mathrm{L}$ after 3 months. For $\mathrm{CsA}+\mathrm{PRED}+\mathrm{AZA}$ recipients, the trough CsA level was $0.10-0.15 \mathrm{mg} / \mathrm{L}$ in the first 3 months after transplantation and $0.05-0.10 \mathrm{mg} / \mathrm{L}$ after 3 months.

\section{Center policy of tissue typing}

Nontransfused patients received at least one leukocyte-poor blood transfusion before transplantation according to the local transfusion protocol, as recently published $^{3}$. Transplants were performed under the auspices of Eurotransplant. Matching priority was HLA-DR, HLA-B, and HLA-A. HLA antigens were considered unacceptable for a patient if antibodies against the specificity had been demonstrated either at the time of transplantation or in the past. Also, mismatches from previous transplants were excluded, as were the paternally inherited antigens of the children of female patients. 
Table 8.1 Characteristics of recipients, donors, and transplantation procedure?

\begin{tabular}{|c|c|c|}
\hline & Mean* & Range" \\
\hline Age recipient & $47.0 \pm 12.79$ & $15.6-72.3$ \\
\hline Gender recipient $(\mathrm{m} / \mathrm{f})$ & $224 / 122$ & $(64.7 \% / 35.3 \%)$ \\
\hline $\begin{array}{l}\text { Cause of renal failure } \\
\text { chronic glomerulonephritis } \\
\text { pyelonephritis } \\
\text { nephrosclerosis } \\
\text { polycystic kidney disease } \\
\text { DM } 1 \\
\text { DM } 2 \\
\text { other }\end{array}$ & $\begin{array}{c}96 \\
28 \\
20 \\
46 \\
19 \\
9 \\
128\end{array}$ & $\begin{array}{l}(27.7 \%) \\
(8.1 \%) \\
(5.8 \%) \\
(13.3 \%) \\
(5.5 \%) \\
(2.6 \%) \\
(37.0 \%)\end{array}$ \\
\hline $\begin{array}{l}\text { Type of renal roplacement therapy pretransplant } \\
\text { hemodialysis } \\
\text { peritoneal dialysis } \\
\text { none }\end{array}$ & $\begin{array}{c}233 \\
108 \\
5\end{array}$ & $\begin{array}{l}(67.3 \%) \\
(31.2 \%) \\
(1.4 \%)\end{array}$ \\
\hline $\begin{array}{c}\text { Transplant no. } \\
1 \\
2 \\
3 \\
4\end{array}$ & $\begin{array}{c}265 \\
64 \\
13 \\
4\end{array}$ & $\begin{array}{l}(76.6 \%) \\
(18.5 \%) \\
(3.8 \%) \\
(1.2 \%)\end{array}$ \\
\hline $\begin{array}{l}\text { PRA ( }(\%) \\
\text { peak serum } \\
\text { current serum }\end{array}$ & $\begin{array}{l}21.4 \pm 1.84 \\
14.0 \pm 1.52\end{array}$ & $\begin{array}{l}0-100 \\
0-100\end{array}$ \\
\hline $\begin{array}{c}\text { Current/neak PRA } \\
0=5 \% \\
6=84 \% \\
85=100 \%\end{array}$ & $\begin{array}{l}256 / 221 \\
65 / 77 \\
25 / 48\end{array}$ & $\begin{array}{l}(74.0 \% / 63.9 \%) \\
(18.8 \% / 22.3 \%) \\
(7.2 \% / 13.9 \%)\end{array}$ \\
\hline Azathioprine $(\mathrm{Y} / \mathrm{N})$ & $71 / 275$ & $(20.5 \% / 79.5 \%)$ \\
\hline $\begin{array}{c}\text { Transplant period } \\
1983-1987 \\
1988-1991 \\
1992-1995\end{array}$ & $\begin{array}{c}125 \\
128 \\
93\end{array}$ & $\begin{array}{l}(36.1 \%) \\
(37.0 \%) \\
(26.9 \%)\end{array}$ \\
\hline Age of donor & $37.5 \pm 16.30$ & $0.0-70.3$ \\
\hline Gender of donor $(\mathrm{m} / \mathrm{n})$ & $213 / 133$ & $(61.6 \% / 38.4 \%)$ \\
\hline Local donor $(\mathrm{Y} / \mathrm{N})$ & $117 / 229$ & $(33.8 \% / 66.2 \%)$ \\
\hline $\begin{array}{l}\text { Type of preservation solution } \\
\text { Eurocollins } \\
\text { UW } \\
\text { HTK } \\
\text { Other (Machine/Betar) }\end{array}$ & $\begin{array}{l}191 \\
102 \\
42 \\
11\end{array}$ & $\begin{array}{l}(55.2 \%) \\
(29.5 \%) \\
(12.1 \%) \\
(3.2 \%)\end{array}$ \\
\hline NHB-donor $(\mathrm{Y} / \mathrm{N})$ & $22 / 324$ & $(6.4 \% / 93.6 \%)$ \\
\hline $\begin{array}{l}\text { Cause of death donor } \\
\text { trauma } \\
\text { CVA } \\
\text { SAB/ICB } \\
\text { Cerobral tumor } \\
\text { Other }\end{array}$ & $\begin{array}{c}168 \\
71 \\
70 \\
4 \\
33\end{array}$ & $\begin{array}{l}(48.6 \%) \\
(20.5 \%) \\
(20.2 \%) \\
(1.2 \%) \\
(9.6 \%)\end{array}$ \\
\hline $\begin{array}{l}\text { WIT (min) } \\
\text { CIT (hours) } \\
\text { WIT2 (min) }\end{array}$ & $\begin{array}{l}4.6 \pm 13.94 \\
29.5 \pm 8.03 \\
35.1 \pm 10.09\end{array}$ & $\begin{array}{c}0-118 \\
2-66 \\
12-90\end{array}$ \\
\hline
\end{tabular}


Mismatch

HLAA $0-\geq 1$

HLAB $0: \geq 1$

HLA-DR $0=\geq 1$

Combined mismatch for HLA-B and -DR

$\begin{array}{ll}\text { B } & \text { DR } \\ 0 & 0 \\ 0 & \geq 1 \\ \geq 1 & 0 \\ \geq 1 & \geq 1\end{array}$

Combined mismatch for HLA.A. HLA-B and -DR

$\begin{array}{llllll}A & B & \text { DR } & & \\ 0 & 0 & 0 & 40 & (11.6) \\ \geq 1 & 0 & 0 & 43 & (12.4) \\ 0 & \geq 1 & 0 & 43 & (12.4) \\ \geq 1 & \geq 1 & 0 & 76 & (22.0) \\ 0 & 0 & \geq 1 & 11 & (3.2) \\ 0 & \geq 1 & \geq 1 & 21 & (6.1) \\ \geq 1 & 0 & \geq 1 & 17 & (4.9) \\ \geq 1 & \geq 1 & \geq 1 & 95 & (27.5)\end{array}$

- Data are given as number or mean $+S D$

- Data ane given as range or perentage 0

+ $\mathrm{CVA}=$ cerebrovasculair accident: $\mathrm{SAD}=$ subarachnoidal bleeding; ICB $=$ intracranial bleeding.

\section{HLA typing}

All kidney donors and recipients were serologically typed for HLA-A, - B, and -DR antigens (broad 1-10). Typing of recipients was performed twice on peripheral blood lymphocytes. Typing of donors was performed on spleen lymphocytes and retyping was done at the Eurotransplant reference laboratory. Antigen splits and crossreactive groups (CREGs) were not considered in evaluating the degree of mismatching for the HLA-A, -B, and -DR antigens between donor and recipient. Recorded were the number of mismatches for each locus, i.e., if the donor was homozygous on the HLA-DR locus for DR1 and the recipient was typed as DR1 DR2, the number of mismatches for HLA-DR was recorded as 0 (zero). The presence of blank alleles in the donor was not excluded. The number of mismatches for HLA-A,- B, and -DR were recoded into 0 and $\geq 1$.

\section{Crossmatch}

Crossmatches in our center included three complement-dependent cytotoxicity (CDC) crossmatches: the standard NIH crossmatch, the two-color fluorescence (TCF) crossmatch, and a dithiotreitol (DTT) crossmatch in which the serum was treated with DTT to reduce IgM antibodies. Sera used for crossmatches at the time of transplant were (1) the current serum drawn at the time of transplantation, (2) the last screened serum sample, and (3) all necessary positive historical samples (peak sera). A negative class 1 crossmatch (NIH) was mandatory for transplantation. This was true for current as well as for historical sera. In the NIH crossmatch, the presence of donor-directed antibodies in the recipient serum was 
defined as cell death $>20 \%$ above background. Screening results were used in the interpretation of the crossmatch.

\section{Antibody screening}

Screening for the presence of anti HLA class I antibodies was performed using the CDC technique with and without the addition of DTT, using a selected panel of 64 lymphocytes. Most HLA antigens were represented three times. The presence of class II antibodies was established at the same time and from the same wells, but with a different positive control for the calculations. Our screening protocol is described in Chapter 3 of this thesis. Class I screening results were expressed as \% PRA for highest historical serum (peak PRA) and serum drawn at the time of transplantation (current PRA). Clinical outcome parameters

Rejection was defined as any rejection treatment in the first 6 months after grafting. In general, rejection episodes were proven by needle core biopsy. Rejection treatment consisted of a 10-day course of rabbit antilymphocyte globulin (RIVM, Bilthoven, the Netherlands) or OKT3-Orthoclone $(\mathrm{n}=3$; Jansen Cilag, Tilburg, the Netherlands). For patients who had received rabbit antilymphocyte globulin in the past or who had a seriously compromised cardiovascular system, the rejection was treated with three doses of methylprednisolon $(0.5-1.0 \mathrm{~g} / \mathrm{dose})$.

Patient failure was defined as death with a functioning graft. If a patient restarted dialysis or was retransplanted due to graft failure, the follow-up ended at the time of graft failure. No exclusions were made.

Graft failure was defined as the loss of kidney function (resumption of dialysis or retransplantation). Death of recipient with a functioning graft was censored. No exclusions were made.

Delayed graft function was defined as the need for dialysis in the first week after transplantation. The first and last dates of dialysis after transplantation were recorded for these patients.

The number of days of hospitalization was defined as from the day of admittance for transplantation until the day of discharge.

Creatinine clearance at year 1 was calculated using the Cockcroft Gault formula $a^{4}$ : creatinine clearance $=$ gender recipient $*(140$-age $) *$ body weight $/(0.81 *$ serum creatinine); for males the correction factor 'gender recipient' had the value 1 , for females 0.85 ; age was reported in years, body weight in $\mathrm{kg}$, and serum creatinine in $\mu \mathrm{mol} / \mathrm{L}$.

All patients were followed up until the date of graft failure, the date of death, or January 1, 1999. 


\section{Statistics}

Statistical analysis was performed using SPSS for Windows, version 7.5.

As a first step, patient, graft, and rejection-free survival were analyzed with the proportional hazards regression analysis (Cox regression), using both stepwise forward selection and backward elimination techniques for all suspected risk factors except number of mismatches for the HLA loci. In case rejection was the outcome, the suspected risk factors tested for included the recipient characteristics: 'age of recipient (years)', 'gender recipient (male vs. female)', 'transplant number $(1+2$ vs. $3+4$ )', 'use of azathioprine (yes vs. no)', '\%PRA (peak and current separately; $0 \%-100 \%$ )', 'original disease (chronic $\mathrm{GN}$-itis vs. pyelonephritis vs. nephrosclerosis vs. PCKD vs. DM1 vs. DM2 vs. other)', 'dialysis modality before transplantation (hemodialysis vs. peritoneal dialysis vs. none)', and 'general evaluation (good vs. moderate vs. poor)'. Included donor characteristics were: 'age of donor (years)', 'gender of donor (male vs. female)', 'cause of death of donor (trauma vs. intracranial bleeding vs. CVA vs. other)', and 'non-heart-beating donor (yes vs. no)'. Included preservation and perioperative characteristics were: 'transplant period (patients divided into tertiles: period 1983-1987, 1988-1991, and 1992-1995)', 'first warm ischemia time (WITl; min; defined as the period from circulatory standstill until start of organ cooling in the donor)', 'second warm ischemia time (WIT2; min; defined as the period at the operation theatre that the graft is taken out of the preservation solution/-machine and time of anastomosis)', 'cold ischemia time (CIT; hours; the period between end of WITI and start of WIT2)', 'type of preservation solution (Eurocollins (EC) vs. University of Wisconsin (UW) vs. histidine-tryptophan-ketoglutarate (HTK) vs. other (machine perfusion/Belzer solution))'. A basic model was tested for, which had to include only direct odds ratio effects that were statistically significant. If two factors were highly associated, the one that was more strongly related to the dependent variable would be chosen. The possibility of interactions between factors in the basic model (different relative effects within different subgroups) was not taken into account since too many additional terms would have to have been included to test for such effects.

With patient and graft survival as outcome variables, suspected risk factors in the basic models were different for the first year(s) and later years. Therefore, patient and graft survival were analyzed separately for these periods. For patient and graft survival, both for the first period and later after transplantation, 'occurrence of acute rejection' and 'DGF' were included as potential risk factors in the model next to the list of risk factors. For patient survival after 4 years and graft survival after 1 year, 'creatinine clearance at year 1 (cockcroft; $\mathrm{mL} / \mathrm{min}$ )', and 'proteinuria at year $1(\mathrm{~g} / 24 \mathrm{~h})^{\prime}$ ' were also included as potential risk factors.

For patient and graft survival, the same policy in model selection was followed as 
for rejection-free survival.

As a second step, for all outcome variables, the mismatch for the HLA loci being studied was used as a single block of risk factors including potential interaction terms between HLA-A, -B, and -DR. A strictly hierarchical model-fitting procedure was followed ${ }^{5}$. If the higher (second grade) interaction term was found to be statistical significant, the data had to be split into subgroups because the effect of mismatch on the outcome parameter was different for the different combinations of HLA loci and one was not allowed to analyze the overall effect of one HLA locus. Only when the higher interaction term was not statistically significant could the lower (first grade) interaction terms be studied and interpreted in the same way. Only when the lower interaction terms turned out not to be statistically significant could the different HLA loci be analyzed as separate risk factors without an additional (synergistic) effect of combinations of mismatches for HLA loci on the outcome parameter.

As a third step, for each outcome variable, risk factors that were included in the basic model were supplemented with the significant HLA locus risk factors to investigate whether mismatch risk factor effects proved to be stable and statistically significant under the potential confounding effects of the risk factors and variables from the basic model in the first step.

Risk factors for DGF, duration of dialysis dependency, duration of hospitalization after transplantation, and creatinine clearance at year 1 were analyzed as dependent variables in logistic and linear regression. For 'DGF' and 'duration of dialysis dependency' as outcome parameters, potential risk factors tested for were the same as those used in the analysis of rejection-free survival in the Cox regression. For 'duration of hospitalization' and 'creatinine clearance at year 1' as outcome parameters, 'occurrence of acute rejection' and 'DGF' were also included as potential risk factors.

Nonparametric tests (Pearson chi-square test, Fisher's exact test, Kruskal-Wallis test) were performed when indicated. A $P$-value $<0.05$ was considered to be statistically significant. 


\section{Results}

\section{Patient survival}

Fifty-one $(14.7 \%)$ of the grafts were lost due to death with a functioning graft during their follow-up. Patient survival is shown in Figure 8.1. In Cox regression analysis, the period of transplantation turned out to be a significant risk factor. Therefore, factors influencing patient survival were analyzed for two separate periods: up to 4 years, and after 4 years post-transplantation.

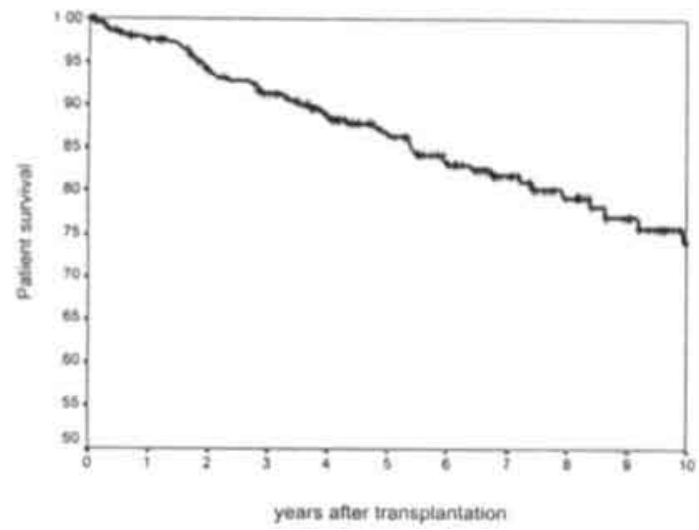

Figure 8.1 Patient survival. Kaplan Meier curve of patient survival of 346 patients transplanted with a cadaveric renal graft af the University Hospital Maastricht. In case of graft failure patients are censored at date of resumption of dialysis or retransplantation.

\section{Patient survival up to $\mathbf{4}$ years}

Significant risk factors in the basic model for patient survival were: age of recipient (seniors at higher risk), recipient of a third or fourth graft, and period of transplantation (decreasing risk with later period of transplantation). The odds ratio to the significant risk factors in the basic model are shown in Table 8.2.

In the analysis of the influence of HLA mismatch (step 2) on patient survival, the second-grade interaction term (HLA-A*HLA-B*HLA-DR) could not be calculated in the maximum likelihood estimation procedure because the information matrix became singular (due to empty cells). In the single risk factor model, the firstgrade interaction term between HLA-B and HLA-DR reached statistical significance. Therefore, data were analyzed separately for 0 and $\geq 1$ mismatch for HLA-B and subsequently in each subgroup for -DR. Only in 0 HLA-B mismatch patients did mismatch for HLA-DR turn out to be a significant risk factor; moreover, only in 0 HLA-DR mismatch patients did mismatch for HLA-B turn out to be a significant risk factor (Table 8.2 ). 
Table 8.2 Cox regression analysis of patient survival (death with functioning graft) up to 4 year after transplantation. Mismatch for HLA-A, -B, and -DR (including interaction terms) is introduced as single risk factor, and in addition to significant risk factors (basic model).

\begin{tabular}{lllll}
\hline Basic modeF & $\boldsymbol{P}$ & df & OR & 95\% CI \\
\hline Age recipient & $<0.0001$ & 1 & 1.12 & $(1.07-1.16)$ \\
Transplant number $3+4$ vs. 1+2 & 0.002 & 1 & 14.21 & $(2.61-77.41)$ \\
Transplant period & 0.01 & 2 & & \\
$\quad 1988-1991$ vs. 1983-1987 & 0.29 & 1 & 0.67 & $(0.32-1.42)$ \\
$1992-1995$ vs. 1983-1987 & 0.003 & 1 & 0.10 & $(0.02-0.45)$ \\
\hline
\end{tabular}

- The basic model for patient death with functioning graft contains the significant risk factors: age of recipient, graft from NHB-donor, and transplant period (chi-square LO 331.96; -2L. chi-square $=41.78 ; 3$ df, $P<0.0001$ ).

\begin{tabular}{|c|c|c|c|c|c|c|c|c|}
\hline \multirow[b]{2}{*}{ Mismatch on } & \multirow[b]{2}{*}{$\boldsymbol{P}$} & \multicolumn{3}{|c|}{ HLA as single risk factor } & \multicolumn{4}{|c|}{ HLA in addition to the basic model } \\
\hline & & df & OR & $95 \% \mathrm{CI}$ & $P$ & df & OR & $95 \% \mathrm{CI}$ \\
\hline HLA-A*HLA-B*HLA-DR & $\approx$ & & & & $=b$ & & & \\
\hline HLA-A ${ }^{*} H L A-B$ & 0.31 & 1 & 2.70 & $(0.38-18.99)$ & 0.12 & 1 & 4.62 & $(0.63-33.74)$ \\
\hline HLA-A*HLA-DR & 0.54 & 1 & 1.75 & $(0.28-10.74)$ & 0.61 & 1 & 1.60 & $(0.25-10.04)$ \\
\hline HLA-B*HLA-DR & 0.05 & 1 & 0.17 & $(0.03-1.09)$ & 0.03 & 1 & 0.13 & $(0.02-0.85)$ \\
\hline HLA-A & 0.87 & 1 & 0.93 & $(0.36-2.36)$ & 0,51 & 1 & 1.38 & $(0.52-3.64)$ \\
\hline if $\mathrm{mm} \mathrm{DR}=0$ & 0.02 & 1 & 4.52 & $(1.01-20.22)$ & 0.05 & 1 & 4.05 & $(0.86-19.12)$ \\
\hline if $\mathrm{mm} D R \geq 1$ & 0.65 & 1 & 0.77 & $(0.25-2.38)$ & 0.43 & 1 & 0.61 & $(0,19-1,97)$ \\
\hline \multirow{2}{*}{ HLA-DR } & 0.02 & 1 & 6.75 & $(1.23-36.94)$ & $0.02^{e}$ & 1 & 8.43 & $(1.46-48.52)$ \\
\hline & 0.76 & 1 & 1.13 & $(0.51-2.52)$ & 0.42 & I & 1.45 & $(0.59-3.56)$ \\
\hline
\end{tabular}

" In the maximum likelihood estimation procedure of the higher interaction term between HLA-A, -B, and DR the information matrix became singular due to empty cells; therefore this item could not be analysed.

- In the maximum likelihood estimation prodedure of the basic model the information matrix became singular due to empty cells; therefore for this item only age recipient was included in basic model.

When HLA mismatch was introduced into a model containing the basic model, the results were similar to those in step 2 (Table 8.2).

\section{Patient survival after 4 years}

Significant risk factors for patient survival were general evaluation (both higher risk for moderate and poor recipients, OR 4.58 and 4.12) and receiving a NHB kidney (OR 15.82; Table 8.3). In the estimation procedure, the information matrix became singular after introduction of the second grade interaction term for HLA mismatch (HLA-A*HLA-B*HLA-DR) as single risk factor and in addition to the basic model (due to empty cells) and, therefore, could not be calculated. Firstgrade interaction terms and mismatch for the individual HLA loci did not contribute significantly to patient survival (Table 8.3). 
Table 8.3 Cox regression analysis of patient survival (death with functioning graft) after 4 years posttransplantation. Mismatch for HLA-A, -B, and -DR (including interaction terms) is introduced as single risk factor, and in addition to significant risk factors (basic model).

\begin{tabular}{lllll}
\hline Basic model & $\boldsymbol{P}$ & df & OR & 95\% CI \\
\hline $\begin{array}{l}\text { NHB donor } \\
\text { General evaluation }\end{array}$ & 0.0008 & 1 & 15.82 & $(3.17-78.94)$ \\
$\quad$ good-> moderate & 0.029 & 2 & & \\
$\quad$ good- $>$ poor & 0.008 & 1 & 4.58 & $(1.49-14.09)$ \\
& 0.21 & 1 & 4.12 & $(0.45-37.72)$ \\
\hline
\end{tabular}

- The basic model for patient death with functioning graft contains the significant risk factors: age of recipient, graft from NHB-donor, and transplant period (chi-square LO 167,$85 ;-2 \mathrm{LL}$ chi-square $=16.13 ; 3$ df, $P<0.0001)$.

\begin{tabular}{|c|c|c|c|c|c|c|c|c|}
\hline \multirow[b]{2}{*}{ Mismatch on } & \multirow[b]{2}{*}{$\boldsymbol{P}$} & \multicolumn{3}{|c|}{ HLA as single risk factor } & \multicolumn{4}{|c|}{ HLA in addition to the basic model } \\
\hline & & df & OR & $95 \% \mathrm{Cl}$ & $P$ & df & OR & $95 \% \mathrm{Cl}$ \\
\hline HLA-A*HLA-B*HLA-DR & $\Rightarrow$ & & & & 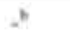 & & & \\
\hline HLA-A*HLA-B & 0.59 & 1 & 0.58 & $(0.08-4.11)$ & 0.72 & 1 & 0.66 & $(0.08-5.86)$ \\
\hline HLA-A*HLA-DR & 0.36 & 1 & 0.45 & $(0.08-2.57)$ & 0.96 & 1 & 0.95 & $(0.13-7.10)$ \\
\hline HLA-B*HLA-DR & 0.66 & 1 & 0.65 & $(0.10-3.53)$ & 0.41 & 1 & 0.39 & $(0.04-3.67)$ \\
\hline HLA-A & 0.16 & 1 & 0.52 & $(0.21-1.26)$ & 0.09 & 1 & 0.42 & $(0.15-1.14)$ \\
\hline HLA-B & 0.91 & 1 & 0.94 & $(0.34-2.61)$ & 0.91 & 1 & 1.07 & $(0.35-3.23)$ \\
\hline HLA-DR & 0.19 & 1 & 1.79 & $(0.75-4.27)$ & 0.07 & I & 2.53 & $(0.89-7.24)$ \\
\hline
\end{tabular}

"In the maximum likelihood estimation procedure of the higher interaction term between HLA-A, -B, and -DR the information matrix became singular due to empty cells; therefore this item could not be analysed.

\section{Graft survival}

A total of 149 of the patients $(43.1 \%)$ lost their graft and were retransplanted or returned to renal replacement therapy during their follow-up. The graft survival is shown in Figure 8.2.

In the Cox regression analysis, significant risk factors in the basic model differed, depending on the time after transplantation. Therefore, graft survival was analyzed separately for the first year after transplantation and from 1 year onwards.

\section{Graft survival up to 1 year}

Cox regression analysis showed that significant risk factors in the first step basic model contributing to graft survival were: DGF (no vs. temporary vs. continuous), \%PRA in peak serum, and duration of WIT2 (Table 8.4). DGF, in particular, was an important risk factor for graft failure within the first year, even when dialysis after transplantation was temporary and graft function was regained (odds ratio $=1.99$ ). 


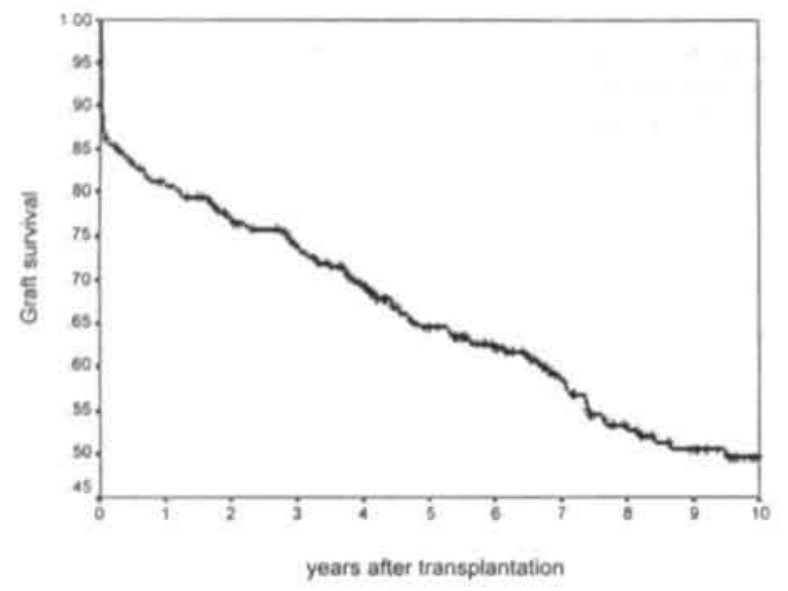

Figure 8.2 Graft survival. Kaplan Meier curve of graft survival of 346 patients transplanted with a cadaveric renal graft at the University Hospital Maastricht. In case of death with function graft patients are censored.

Mismatches for HLA-A, HLA-B, and HLA-DR did not contribute in a statistically significant way to graft survival, either when regarded as a single risk factor or when the basic model was included (Table 8.4). Because the first grade interaction term between HLA-A and HLA-DR reached statistical significance in the model already containing the basic model (step 3 ), data were also analyzed while they were split according to both factors in the interaction term being studied. The results of this analysis showed that neither mismatch for HLA-DR nor mismatch for HLA-A contributed significantly to graft failure under control of the other locus $(P=0.06)$.

\section{Graft survival after 1 year}

Cox regression analysis showed that the significant risk factors in the basic model contributing to graft survival were: creatinine clearance at 1 year, the level of proteinuria at 1 year, and the age of recipient (Table 8.5). Each decrease in creatinine clearance by $1 \mathrm{~mL} / \mathrm{min}$ increased the risk of failure by $6 \%$, while each gram of protein in the $24 \mathrm{~h}$ urine collection increased the risk of failure by $31 \%$. Each year increase in the age of the recipient decreased the risk of graft failure by $3 \%$.

Neither the second-grade nor the first-grade interaction term was statistically significant, either when introduced as a single risk factor or when added to the basic model (Table 8.5). Mismatch for HLA-B contributed to graft failure when introduced as a single risk factor $(\mathrm{OR} 1.81, P=0.02)$ but did not reach statistical significance when introduced into the basic model (OR 1.15, $P=0.62$ ). 
Table 8.4 Cox regression analysis of 1-year graft survival". Mismanch for HLA-A, -B, and -DR (including interaction terms) is introduced as single risk factor, and in addition to significant risk factors (basic model).

\begin{tabular}{lccll}
\hline Basic model $^{\circ}$ & $\boldsymbol{P}$ & df & OR & $95 \% \mathrm{CI}$ \\
\hline DGF & $<0.0001$ & 2 & & \\
Yes temporary vs, no & 0.016 & 1 & 1.99 & $(1.14-3.47)$ \\
Contimuous vs. no & $<0.0001$ & 1 & 18.12 & $(8.32-38.44)$ \\
& & & & \\
PRA peak-serum & 0.028 & 1 & 1.007 & $(1.001-1.013)$ \\
WIT2 & $<0.0001$ & 1 & 1.005 & $(1.003-1.008)$ \\
\hline
\end{tabular}

- The basic model for 1-year graft survival contains the significant risk factors: the occurrence of DGF, PRA peak-serum, and WIT2 (chi-square LO 758.316; -2L chi-square $=51.952 ; 4$ df, $P<0.0001$ ).

\begin{tabular}{|c|c|c|c|c|c|c|c|c|}
\hline \multirow[b]{2}{*}{ Mismatch on } & \multirow[b]{2}{*}{$P$} & \multicolumn{3}{|c|}{ HLA as single risk factor } & \multicolumn{4}{|c|}{ HLA in addition to the basic model } \\
\hline & & df & OR & $95 \% \mathrm{CI}$ & $\boldsymbol{P}$ & df & OR & $95 \% \mathrm{CI}$ \\
\hline HLA-A*HLA-B*HLA-DR & 0.13 & 1 & 7.07 & $(0.59-85.06)$ & 0.10 & 1 & 9.06 & $(0.72-113.62)$ \\
\hline HLA-A*HLA-B & 0.08 & 1 & 0.38 & $(0.13-1.15)$ & 0.12 & 1 & 0.41 & $(0.13-1.27)$ \\
\hline HLA-A*HLA-DR & 0.17 & 1 & 2.13 & $(0.71-6.33)$ & 0.05 & 1 & 2.96 & $(0.98-8.94)$ \\
\hline HLA-B*HLA-DR & 0.34 & 1 & 1.81 & $(0.53-6.26)$ & 0.42 & 1 & 1.68 & $(0.47-6.05)$ \\
\hline HLA-A & 0.25 & 1 & 0.74 & $(0.45-1.23)$ & 0.45 & 1 & 0.82 & $(0.49-1.36)$ \\
\hline HLA-B & 0.63 & 1 & 1.14 & $(0.66-1.66)$ & 0.85 & 1 & 0.95 & $(0.54-1.65)$ \\
\hline HLA-DR & 0.18 & 1 & 1.39 & $(0.86-2.25)$ & 0.38 & 1 & 0.38 & $(0.76-2.04)$ \\
\hline if $\mathrm{mm} \mathrm{DR}=0$ & 0.12 & 1 & 0.58 & $(0.30-1.14)$ & 0.06 & 1 & 0.51 & $(0.26-1.02)$ \\
\hline if $\mathrm{mm} \mathrm{DR} \geq 1$ & 0.91 & 1 & 1.05 & $(0.45-2.43)$ & 0.30 & 1 & 1.59 & $(0.65-3.91)$ \\
\hline HLA-DR if $\mathrm{mm} A=0$ & 0.97 & 1 & 1.02 & $(0.43-2.44)$ & 0.55 & 1 & 0.76 & $(0.31-1.89)$ \\
\hline if $\mathrm{mm} A \geq 1$ & 0.06 & 1 & 1.79 & $(0.96-3.36)$ & 0.06 & 1 & 1.84 & $(0.97-3.49)$ \\
\hline
\end{tabular}

- Cox regression analysis in 346 recipients: suspected risk factors recipient age, gender recipient, peak PRA or current PRA, transplant number, original disease. type of renal replacement therapy before transplantation, use of azathioprine, general evaluation recipient, gender donor, donor age, NHB donor, local donor, type of preservation solution, WIT1, CIT, WIT2, mismatch for HLA-A, -B, -DR (including interaction terms), occurrence of rejection, DGF, transplant period.

The final model results are shown including only statistically significant risk factor. Risk factors with more than two categories are broken down to indicator contrasts.

Graft failure $=$ restart dialysis or retransplant; death with functioning graft was censored.

\section{Rejection}

A total of 131 of the patients $(37.9 \%)$ were treated for acute rejection. The rejection-free survival is shown in Figure 8.3.

Cox regression analysis showed that significant risk factors in the basic model contributing to rejection were \%PRA in peak serum, CIT, and transplant number $(3+4$ vs. $1+2$; Table 8.6$)$. An increase in PRA or transplant number resulted in a higher risk for rejection, while an increase in CIT resulted in a lower risk for rejection. Compared to local kidneys, exchanged (non-local) kidneys have a longer CIT but a better HLA match. To investigate whether the correlation of longer CIT with lower rejection rate was an artificial effect, only recipients of non-local 
kidneys were studied. Within this restricted analysis, CIT was still a significant risk factor, thereby excluding the abovementioned potential artefact.

Table 8.5 Cox regression analysis of graft survival* after year 1 of 273 renal transplant recipients. Mismatch for HLA-A, - B, and -DR (including interaction terms) is introduced as single risk factor, and in addition to significant risk factors (basic model).

\begin{tabular}{lcccc}
\hline Basic model & $\boldsymbol{P}$ & df & OR & 95\% CI \\
\hline Creatinine clearance year I & $<0.0001$ & 1 & 0.94 & $(0.924-0.957)$ \\
Proteimuria year 1 & 0.0001 & 1 & 1.31 & $(1.140-1.495)$ \\
Age of recipient & 0.0005 & 1 & 0.97 & $(0.955-0.987)$ \\
\hline
\end{tabular}

- The basic model of graft survival after 1 year contains the significant risk factors: creatinine clearance at year 1, proteinuria at year 1 , and age of recipient (chi-square LO 848.429; -2LL chi-square $=81.068$;

3 df, $P<0.0001)$.

\begin{tabular}{lcccccccc}
\hline & \multicolumn{4}{c}{ HLA as single risk factor } & \multicolumn{3}{c}{ HLA in addition to the basic model } \\
Mismatch on & $P$ & df & OR & $95 \%$ CI & $P$ & df & OR & $95 \%$ CI \\
\hline HLA-A*HLA-B*HLA-DR & 0.66 & 1 & 1.75 & $(0.14-21.43)$ & 0.67 & 1 & 0.58 & $(0.04-7.42)$ \\
HLA-A*HLA-B & 0.90 & 1 & 0.93 & $(0.31-2.75)$ & 0.43 & 1 & 1.56 & $(0.51-4.74)$ \\
HLA-A*HLA-DR & 0.94 & 1 & 1.04 & $(0.38-2.86)$ & 0.57 & 1 & 1.35 & $(0.47-3.82)$ \\
HLA-B*HI_A-DR & 0.93 & 1 & 0.94 & $(0.28-3.21)$ & 0.62 & 1 & 0.73 & $(0.21-2.49)$ \\
HLA-A & 0.39 & 1 & 0.81 & $(0.50-1.31)$ & 0.38 & 1 & 0.81 & $(0.51-1.29)$ \\
HLA-B & 0.02 & 1 & 1.81 & $(1.08-3.06)$ & 0.62 & 1 & 1.15 & $(0.66-1.98)$ \\
HL_A-DR & 0.95 & 1 & 1.02 & $(0.69-1.59)$ & 0.99 & 1 & 1.00 & $(0.64-1.59)$ \\
\hline
\end{tabular}

- Cox regression analysis in 273 recipients: suspected risk factors recipient age, gender recipient, peak PRA or current PRA, transplant number, original disease, type of renal replacement therapy before transplantation, use of azathioprine, general evaluation recipient, gender donor, donor age, NHB donot, local donor, type of preservation solution, WIT1, CIT, WIT2, mismatch for HLA-A, -B, -DR (including interaction terms), occurrence of rejection, DGF, creatinine clearance at year 1, proteinuria at year $\mathrm{I}$.

The final model results are shown including only statistically significant risk factor. Risk factors with more than two categories are broken down to indicator contrasts.

Graft failure = restart dialysis or retransplant: death with functioning graft was censored.

Because the second-grade interaction term between HLA-A, -B, and -DR was statistically significant, both when introduced as a single factor and when added to the basic model, the data were analyzed while they were split according to the mismatch combinations for HLA-A, -B, and -DR. These results are shown in Table 8.6. HLA-DR mismatch turned out to be a significant risk factor for rejection only in the 0 HLA-A and -B mismatch group and in the $\geq 1$ HLA-A and $\geq 1$ HLA-B mismatch group. HLA-B mismatch turned out to be a significant risk factor for rejection only in the 0 HLA-A and -DR mismatch group. In contrast, HLA-A mismatch was a significant protective factor for rejection in the $\geq 1$ HLA-B and 0 HLA-DR mismatch group. In the other groups, mismatch for none of the HLA loci was a significant risk factor contributing to rejection. These results were very similar when the basic model risk factors were included. 


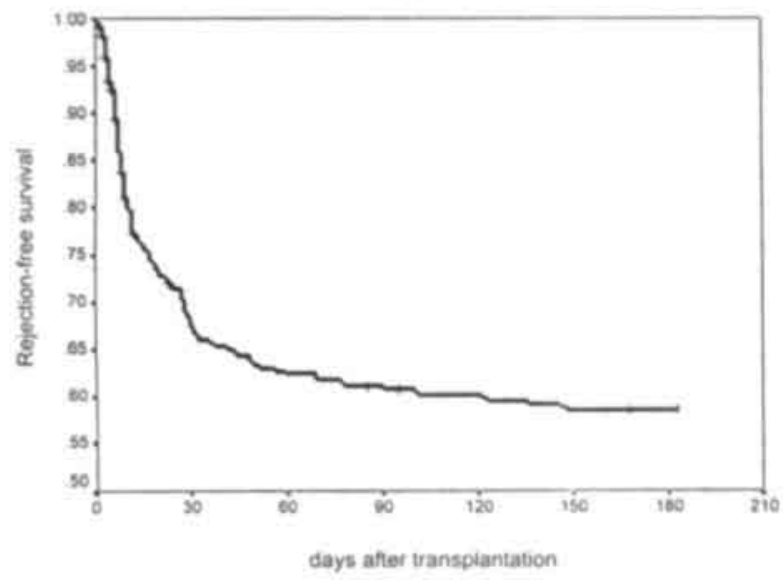

Figure 8.3 Rejection-free survival. Kaplan Meier curve of rejection-free survival of 346 patients transplanted with a cadaveric renal graft at the University Hospital Maastricht. In case of death or graft loss patients are censored.

\section{Delayed graft function (DGF) and duration of DGF}

Delayed graft function was defined as the need for dialysis in the first week after transplantation. A total of 164 of the patients $(47.4 \%)$ had DGF. Twelve of them had a never-functioning graft, while 152 patients eventually regained graft function and dialysis could be stopped at a median of 12 days after transplantation (range 1116 days).

By logistic regression analysis, potential donor, recipient, and transplant variables predicting DGF were analyzed. Risk factors that turned out to be statistically significant were: type of pretransplant dialysis treatment (HD more at risk than CAPD), general evaluation score ('poor' more at risk), gender of recipient (male), longer period of preservation (for both CIT and WIT1) and high \% PRA in peak serum (Table 8.7). With these factors the model's explained variance was $11.7 \%$ with $64.8 \%$ of the DGF correctly predicted $\left(-2 \mathrm{LL} \mathrm{X} \mathrm{X}^{2} 0=399.12\right.$, improvement of the model $\left(-2 \mathrm{LL} \mathrm{X} \mathrm{X}^{2}\right.$-change $\left.)=52.85 ; 8 \mathrm{df}, P<0.0001\right)$. Other potential risk factors, including mismatch for any HLA locus or occurrence of acute rejection, had no significant relation to the (non)occurrence of DGF. 
Table 8.6 Cox regression analysis of rejection-free survival". Mismatch for HLA-A, -B, and -DR (including interaction terms) is introduced as single risk factor, and in addition to significant risk factors (basic model).

\begin{tabular}{lcccc}
\hline Basic model & $P$ & df & OR & 95\% CI \\
\hline PRA peak-serum & $<0.0001$ & 1 & 1.01 & $(1.006-1.015)$ \\
CrT & 0.029 & 1 & 0.97 & $(0.951-0.997)$ \\
Transplant no 3/4 vs. 1/2 & 0.022 & 1 & 2.12 & $(1.113-4.027)$ \\
\hline
\end{tabular}

b The basic model for rejection-free survival contains the significant risk factors: PRA peak-serum, CIT, age of donor, and transplant number $(1+2$ vs, $3+4)$, (chi-square LO 1446.30; $-2 L \mathrm{~L}$ chi-squarechange $=27,42 ; 3$ df, $P<0.0001$ ).

\begin{tabular}{|c|c|c|c|c|c|c|c|c|}
\hline \multirow[b]{2}{*}{ Mismatch on } & \multirow[b]{2}{*}{$P$} & \multicolumn{3}{|c|}{ HLA as single risk factor } & \multicolumn{4}{|c|}{ HLA in addition to the basic model } \\
\hline & & df & OR & $95 \% \mathrm{CI}$ & $P$ & df & OR & $95 \% \mathrm{Cl}$ \\
\hline HLA-A*HLA-B*HLA-DR & 0.03 & 1 & 6.19 & $(1.22-31.50)$ & 0.02 & 1 & 7.27 & $(1.39-37.93)$ \\
\hline \multicolumn{9}{|c|}{$\begin{array}{l}\text { Mismatch for HLA-DR } \\
\text { under control of HLA-A and -B }\end{array}$} \\
\hline $\begin{array}{l}m m A=0 \mathrm{mmB}=0 \\
\mathrm{mmA}=0 \mathrm{mmB} \geq 1 \\
\mathrm{mmA} \geq 1 \mathrm{mmB}=0 \\
\mathrm{mmA} \geq 1 \mathrm{mmB} \geq 1\end{array}$ & $\begin{array}{l}0.02 \\
0.77 \\
0.73 \\
0.02\end{array}$ & $\begin{array}{l}1 \\
1 \\
1 \\
1\end{array}$ & $\begin{array}{l}3.40 \\
0.90 \\
1.17 \\
1.82\end{array}$ & $\begin{array}{l}(1.23-9.45) \\
(0.44-1.84) \\
(0.47-2.91) \\
(1.08-3.07)\end{array}$ & $\begin{array}{l}0.05 \\
0.67 \\
0.89 \\
0.03\end{array}$ & $\begin{array}{l}1 \\
1 \\
1 \\
1\end{array}$ & $\begin{array}{l}2.83 \\
0.85 \\
1.08 \\
1.77\end{array}$ & $\begin{array}{l}(1.01-7.95) \\
(0.41-1.77) \\
(0.35-3.31) \\
(1.05-3.01)\end{array}$ \\
\hline \multicolumn{9}{|c|}{$\begin{array}{l}\text { Mismatch for HLA-B } \\
\text { under control of HLA-A and -DR }\end{array}$} \\
\hline $\begin{array}{l}\operatorname{mm} A=0 \quad m m D R=0 \\
m m A=0 \quad m m D R \geq 1 \\
m m A \geq 1 \quad m m D R=0 \\
m m A \geq 1 \quad m m D R \geq 1\end{array}$ & $\begin{array}{l}0.002 \\
0.67 \\
0.41 \\
0.71\end{array}$ & $\begin{array}{l}1 \\
1 \\
1 \\
1\end{array}$ & $\begin{array}{l}3.26 \\
0.81 \\
0.75 \\
1.16\end{array}$ & $\begin{array}{l}(1.45-7.31) \\
(0.31-2.10) \\
(0.38-1.47) \\
(0.52-2.60)\end{array}$ & $\begin{array}{l}0.01 \\
0.43 \\
0.39 \\
0.54\end{array}$ & $\begin{array}{l}1 \\
1 \\
1 \\
1\end{array}$ & $\begin{array}{r}12.90 \\
0.67 \\
0.72 \\
1.29\end{array}$ & $\begin{array}{l}(1.22-6.90) \\
(0.26-1.78) \\
(0.34-1.52) \\
(0.56-2.94)\end{array}$ \\
\hline \multicolumn{9}{|c|}{$\begin{array}{l}\text { Mismatch for HLA-A } \\
\text { under control of HLA-B and -DR }\end{array}$} \\
\hline $\begin{array}{l}\mathrm{mmB}=0 \mathrm{mmDR}=0 \\
\mathrm{mmB}=0 \mathrm{mmDR} \geq 1 \\
\mathrm{mmB} \geq \mathrm{mmDR}=0 \\
\mathrm{mmB} \geq \mathrm{mmDR} \geq\end{array}$ & $\begin{array}{l}0.19 \\
0.39 \\
0.003 \\
0.55\end{array}$ & $\begin{array}{l}1 \\
1 \\
1 \\
1\end{array}$ & $\begin{array}{l}1.76 \\
0.63 \\
0.41 \\
0.81\end{array}$ & $\begin{array}{l}(0.74-4.21) \\
(0.22-1.80) \\
(0.23-0.73) \\
(0.42-1.59)\end{array}$ & $\begin{array}{l}0.26 \\
0.43 \\
0.01 \\
0.68\end{array}$ & $\begin{array}{l}1 \\
1 \\
1 \\
1\end{array}$ & $\begin{array}{r}1.67 \\
0.63 \\
10.45 \\
0.86\end{array}$ & $\begin{array}{l}(0.68-4.13) \\
(0.20-1.98) \\
(0.25-0.83) \\
(0.43-1.71)\end{array}$ \\
\hline
\end{tabular}

- Cox regression analysis in 346 recipients: suspected risk factors recipient age, gender recipient, peak PRA or current PRA, transplant number (recoded: $1+2$ vx. $3+4$ ), original disease, type of renal replacement therapy before transplantation, use of azathioprine, general evaluation recipient, gender donor, donor age. NHB donor, type of preservation solution, WIT1, CIT, WIT2, mismatch for HLA-A, -B, -DR (including interaction terms).

The final model results are shown including only statistically significant risk factors. Risk factors with more than two categories are broken down to indicator contrasts. 
Table 8.7 Results of logistic regression analysis with as outcome 'occurrence of DGF' $(\mathrm{n}=346)$

\begin{tabular}{|c|c|c|c|c|c|c|}
\hline Risk factor & b & seb & df & $P$ & $\exp (\mathbf{b})$ & $95 \% \mathrm{Cl}$ of $\mathrm{b}$ \\
\hline General evaluation & & & 2 & 0.016 & & \\
\hline good $\rightarrow>$ moderate & 0.28 & 0.26 & 1 & 0.272 & 1.32 & $0.80<->2.18$ \\
\hline good $->$ poor & 1.46 & 0.52 & 1 & 0.005 & 4.29 & $1.56<->11.78$ \\
\hline $\mathrm{CrT}$ & 0.04 & 0.02 & 1 & 0.020 & 1.04 & $1.01<->1.07$ \\
\hline WIT1 & 0.05 & 0.02 & $i$ & 0.003 & 1.05 & $1.02<->1.09$ \\
\hline Type of dialysis pretransplant & & & 2 & 0.188 & & \\
\hline CAPD $\rightarrow$ HD & 0.45 & 0.26 & I & 0.087 & 1.56 & $0.94<->2.61$ \\
\hline CAPD -> none & -5.87 & 9.64 & 1 & 0.543 & 0.003 & $0.000<->454388$ \\
\hline HD $->$ none & -6.33 & 9.68 & 1 & 0.51 & 0.002 & $0.00<->312992$ \\
\hline Male recipient & 0.59 & 0.27 & I & 0.027 & 1.81 & $1.07<->3.06$ \\
\hline PRA peak $(\%)$ & 0.01 & 0.004 & 1 & 0.010 & 1.01 & $1.002<->1.089$ \\
\hline Constant & -2.45 & 0.57 & 1 & $<0.0001$ & & \\
\hline Constant & -1.89 & 0.53 & 1 & 0.00 & & \\
\hline
\end{tabular}

Model improvement: 52.85 by 8 df, $P<0.0001$. Explained variance: $11.7 \% ; 64.8 \%$ correctly predicted.

" constant for HD -> none (constant for HD -> CAPD = negative value of CAPD $->$ HD).

Factors correlated with 'duration of dialysis dependency' were analyzed for the 152 patients with temporary DGF by linear regression analysis. Because of a skewed frequency distribution, the data were log-transformed. The significant risk factors contributing to the duration of dialysis dependency were gender of donor (female) and age of donor (Table 8.8). Mismatch for none of the HLA loci was a significant predictive factor for the duration of dialysis dependency.

Table 8.8 Results of linear regression analysis with as outcome duration of dialysis dependency" $(n=152)$.

\begin{tabular}{lccccc}
\hline Risk factor & b & seb & beta & t & sign. of t \\
\hline Female donor & 0.234 & 0.074 & 0.247 & 3.15 & 0.002 \\
Age donor & 0.005 & 0.002 & 0.193 & 2.46 & 0.015 \\
Constant & 0.550 & 0.125 & & 4.40 & $<0.0001$ \\
\hline
\end{tabular}

$\mathrm{R}^{2}=0.115$, Model F-ratio $=9.65$ at 2 and $149 \mathrm{df} ; P=0.0001$

'Duration of dialysis dependency (days) is logtransformed because of non-normal distribution.

\section{Duration of hospitalization}

After transplantation 51 patients $(14.7 \%)$ lost their graft function $(n=50)$ or died with a functioning graft $(n=1)$ before discharge; these patients were excluded from this analysis. One patient had a missing date of discharge and was excluded from analysis. The median duration of hospitalization was 26 days (range 13-132 days; $\mathrm{n}=294$ ). Risk factors that influenced duration of hospitalization were analyzed by 
linear regression analysis. Because of a positive skewed frequency distribution in the duration, the data were log-transformed. The results of this regression analysis are shown in Table 8.9. Significant risk factors related to an increased duration of hospitalization were (in decreasing importance): DGF, occurrence of acute rejection, transplant in the second or third period (1987-1991 and 1992-1995), age of donor (senior ones at risk), \%PRA in peak serum (higher more at risk), and age of recipient (seniors more at risk).

Table 8.9 Results of linear regression analysis with as outcome 'duration of hospitalization after transplantation' $(\mathrm{n}=294)^{*}$.

\begin{tabular}{|c|c|c|c|c|c|c|c|}
\hline Riskfactor & & b & seb & beta & $t$ & sign of $t$ & $95 \% \mathrm{CI}$ of $\mathrm{b}$ \\
\hline DGF & & 0.172 & 0.018 & 0.435 & 9.50 & $<0.0001$ & $0.135<->0.208$ \\
\hline Acute rejection & & 0.132 & 0.019 & 0.327 & 6.96 & $<0.001$ & $0.095<->0.170$ \\
\hline Age donor & & 0.002 & 0.001 & 0.154 & 3.36 & 0.001 & $0.001<->0.003$ \\
\hline Age recipient & & 0.002 & 0.001 & 0.121 & 2.62 & 0.009 & $<0.001<->0.003$ \\
\hline \multirow{3}{*}{ Transplant period } & $2 v \times 1$ & 0.067 & 0.021 & 0.165 & 3.22 & 0.001 & $0.026<->0.108$ \\
\hline & 3 vs. 1 & 0.043 & 0.023 & 0.096 & 1.86 & 0.064 & $-0.002<->0.089$ \\
\hline & 3 v. $2^{b}$ & -0.024 & 0.023 & -0.053 & -1.04 & 0.301 & $-0.069<->0.022$ \\
\hline PRA peak (\$) & & 0.00074 & 0.0003 & 0.125 & 2.67 & 0.008 & $<0.001<->0.001$ \\
\hline Constant & & 1.119 & 0.042 & - & 26.62 & $<0.001$ & $1.036<->1.202$ \\
\hline Constant" & & 1.186 & 0.042 & - & 28.39 & $<0.001$ & $1.104<->1.269$ \\
\hline
\end{tabular}

- Duration of dialysis dependency (days) is logtransformed because of non-normal distribution. $\mathrm{n}=294$ ( 52 patients graft failure or dying during hospitalization).

$\mathrm{R}^{2}=0.412$, Model F-Ratio $=28.67$ at 7 and 286 df; $P<0.0001$.

" constant for transplant period 3 vs. 2 (constant for transplant period 2 vs. $1=$ negative value of 1 vs. 2)

\section{Creatinine clearance at 1 year after transplantation}

Out of 346 transplant patients, 73 had graft failure during the first year after transplantation. The mean creatinine clearance for the 273 patients with a functioning graft was $49 \mathrm{~mL} / \mathrm{min}$ (SD: 18.8, range: 9-105). For these patients, a linear regression analysis was performed to determine statistically significant risk factors related to creatinine clearance. Three patients had to be excluded because data on risk factors shown to be significant were missing. The results of this analysis on the 270 remaining patients are shown in Table 8.10.

Risk factors resulting in significantly lower creatinine clearance were: age of recipients (seniors more at risk), pretransplant treatment by peritoneal dialysis, age of donors (seniors more at risk), preservation of the graft with Eurocollins instead of UW or HTK, longer CIT, mismatch for HLA-B, and longer duration of hospitalization. One factor related to a higher creatinine clearance was DM2 as 'cause of renal failure'. Neither DGF nor rejection was related to creatinine clearance at I year after transplantation. 
Table 8.10 Results of linear regression analysis with as outcome 'creatinine clearance at I year after transplantation".

\begin{tabular}{lcccccc}
\hline Risk factor & $\mathbf{b}$ & seb & beta & $\mathbf{t}$ & sign. of $\mathbf{t}$ & $\mathbf{9 5 \%} \mathbf{C l}$ of b \\
\hline Age recipient & -0.24 & 0.08 & -0.16 & -3.02 & 0.003 & $-0.40<->-0.08$ \\
HD -> CAPD & 5.73 & 2.14 & 0.14 & 2.67 & 0.008 & $1.51<->9.95$ \\
HD -> none & 3.90 & 7.29 & 0.03 & 0.54 & 0.593 & $-10.45<->18.26$ \\
& & & & & & \\
Other-Chron. GN-itis & 4.47 & 2.48 & 0.11 & 1.80 & 0.073 & $-0.42<->9.36$ \\
Other-pyelonephritis & 3.91 & 4.09 & 0.05 & 0.96 & 0.339 & $-4.13<->11.96$ \\
Other-nefrosclerosis & 4.75 & 4.73 & 0.05 & 1.00 & 0.317 & $-4.58<->14.07$ \\
Other-PCKD & 4.63 & 3.15 & 0.09 & 1.47 & 0.143 & $-1.58<->10.83$ \\
Other-DM1 & 7.68 & 4.20 & 0.10 & 1.83 & 0.069 & $-0.60<->15.95$ \\
Other-DM2 & 18.53 & 6.33 & 0.16 & 2.93 & 0.004 & $6.07<->30.99$ \\
& & & & & & \\
CIT & -0.31 & 0.12 & -0.14 & -2.52 & 0.012 & $-0.55<->-0.07$ \\
& & & & & & \\
EC - > UW & 12.64 & 2.22 & 0.31 & 5.70 & $<0.001$ & $8.27-17.01$ \\
EC - $>$ HTK & 13.05 & 3.12 & 0.23 & 4.19 & $<0.001$ & $6.91<->19.19$ \\
EC - $>$ Machine & 15.22 & 8.10 & 0.10 & 1.88 & 0.061 & $-0.73<->31.17$ \\
& & & & & & \\
Age donor & -0.42 & 0.06 & -0.36 & -6.54 & $<0.001$ & $-0.54<->-0.29$ \\
HLA-B mismatch & -4.28 & 2.13 & -0.11 & -2.01 & 0.046 & $-8.48<->-0.08$ \\
Hospital stay & -0.16 & 0.06 & -0.14 & -2.61 & 0.009 & $-0.27<->-0.04$ \\
& & & & & & \\
Constant & 81.49 & 5.93 & & 13.75 & $<0.001$ & $69.82<->93.16$ \\
\hline
\end{tabular}

* $\mathrm{n}=270$ ( 3 missing: 73 system missing (no 1 year graft function).

$\mathrm{R}^{2}=0.344$, Model F-Ratio $=8.29$ at 16 and $253 \mathrm{df}: P<0.0001$

\section{Discussion}

Factors related to renal graft outcome have been reported in single center studies ${ }^{6-8}$ and in studies performed by transplant registries such as Eurotransplant, UCLA, and $\mathrm{CTS}^{9-14}$. Registry studies have the advantage of being able to include large numbers of patients. However, the disadvantage of analysing a heterogenous patient group from different centers is the 'noise' of center effects. Center effect is a major factor in renal transplant outcome ${ }^{15}$. Therefore, single-center studies, using a more uniform patient group and management, may be valuable in the analysis of transplant outcome, especially of outcome parameters other than patient and graft survival.

We analyzed all cadaveric renal transplantations performed in the University Hospital Maastricht using cyclosporine $\mathrm{A}$ as basic immunosuppression in the period 1983 through 1995. All patients were treated according to the same 
immunosuppressive protocol, and both patient management and senior staff remained essentially unchanged during this period. From this perspective, this constitutes a homogeneous study population. Parameters potentially related to renal graft outcome were analyzed using multivariate techniques.

\section{Patient survival}

Overall patient survival (censored for graft failure) in the first year after transplantation was $\pm 95 \%$, decreasing to $\pm 85 \%$ at year 5 and $\pm 75 \%$ at year 10 . Factors of statistical significance in patient survival were different for the period up to and beyond 4 years post-transplantation. Significant risk factors related to patient survival within 4 years were: transplant period, age of recipient, and transplantation of a third or fourth graft. Patients who underwent transplantation during the years 1991-1995 (the latest period) had a protective odds ratio of 0.10 compared to patients who did so during the years 1983-1987 (the first period). This might have been due to improvement in patient selection, perioperative care, and management of comorbidity, such as cardiovascular diseases and risk factors. Cardiovascular diseases have a high prevalence in the transplant population ${ }^{16}$ and they form the main cause of death in this population ${ }^{16.17}$. In the present study, where $45 \%$ of the recipients were over 50 years of age and $18 \%$ were over 60 years of age, the death score increased by $12 \%$ for every year of increase in the age of the recipient. With an increase in the age of the recipient at the time of transplantation, death with a functioning graft will become more and more important. Although only $5 \%$ of our patients received more than two transplants, they are at a very high risk of death within 4 years (odds ratio 14). This increased risk of dying with a functioning graft might be related to adverse effects of previous exposure to immunosuppressive medication (e.g., hypertension, hyperlipemia and carcinogenicity) and more comorbidity than first or second transplants. Compared to patients receiving their first or second graft, third and fourth graft recipients significantly more often used azathioprine $(18.2 \%$ vs. $64.7 \%$ $P=0.001)$ and needed treatment for acute rejection $(36.2 \%$ vs. $70.6 \%, P=0.01)$. They also had a trend towards more frequent DGF (65\% vs. $47 \%)$ and chronic glomerulonephritis as their original disease $(47 \%$ vs. $27 \%)$, and they less often had a good general evaluation ( $40 \%$ vs. $54 \%$ ). Thus, a set of incidental factors, each with a potentially negative relation to patient survival, existed simultaneously in these patients.

Patients with a mismatch for either HLA-B or -DR were at a significantly higher risk of dying up to 4 years after transplantation than patients without any mismatch for HLA-B and -DR in bivariate as well as multivariate analysis $(P=0.02-0.05$; odds ratio 4-8). This cannot be explained by differences in donor and patient characteristics or by differences in rejection rate between the different mismatch 
groups (data not shown).

Factors related to death with a functioning graft in the period after 4 years posttransplantation differed from those within 4 years: general evaluation (including comorbidity, age, and transplant history) and receiving a graft from a NHB donor. Because general evaluation is based on comorbidity, age, and transplant history, two of the three factors shown to be risk factors for patient survival early $(<4$ years) after transplantation are indirectly also risk factors for patient survival after 4 years. The relationship between receiving a graft from a NHB donor and increased risk of dying more than 4 years after transplantation has not been reported before. In bivariate analysis, transplants with a NHB donor graft differed significantly only for the following parameters of Table 1 from transplants with a HB donor graft: more often from a local donor $(P=0.001)$, longer WITI $(P=0.007)$, fewer 0 mismatches for HLA-A and -DR $(P=0.02$ and $P=0.01$, respectively), and higher donor age $(P=0.007)$. Differences in recipient characteristics were small and not statistically significant at all. The clinical outcome differed significantly by a higher frequency of DGF $(P=0.004)$, but not by the occurrence of acute rejection or creatinine clearance at year 1 . We have to bear in mind that the number of grafts from NHB donors was relatively small $(\mathrm{n}=22)$. Studies including more non-heart-beating donors than this study have shown that graft and patient survival are comparable in patient receiving grafts from NHB and heartbeating cadaveric donors ${ }^{18,19}$. Therefore, the correlation of receiving a graft from a NHB donor with patient death might have been due to the small sample size.

Mismatching for HLA loci did not contribute significantly to the abovementioned risk factors for late patient survival.

\section{Graft survival}

Overall graft survival (censored for death with functioning graft) in the first year after transplantation was $\pm 80 \%$, decreasing to $\pm 65 \%$ at year 5 and $\pm 50 \%$ at year 10. Because Cox regression analysis showed that significant risk factors differed for the 1-year graft survival and graft survival after 1 year, graft survival was analyzed separately for these periods.

Risk factors for graft failure within 1 year included the immunological factor peak PRA. For graft survival, \%PRA of both peak and current serum were equally strong risk factors and had a high positive correlation with each other. Introducing both factors into the model did not improve the model. As reported before, DGF increased the risk of graft failure. This was found not only for those patients who had to continue dialysis (never-functioning grafts; odds ratio 18.12); also patients who regained graft function and became dialysis-independent were at a doubled risk 
of graft failure within the first year compared to patients with an immediately functioning graft. The cause of DGF is multifactorial and related to immunologic as well as recipient and donor factors. Increased anastomosis time (WIT2) was the third risk factor for graft loss. This might have been due to technical problems during the operation that increased the risk of thrombosis or due to increased ischemia/reperfusion damage due to the longer WIT2. Neither mismatch for any HLA locus nor occurrence of acute rejection was a risk factor for graft loss within the first year after transplantation. Recurrent acute rejections $\mathrm{s}^{6,20}$ and the vascular type of acute rejection ${ }^{21}$ have, in particular, been shown to be risk factors for graft loss. We used polyclonal antilymphocyte antibodies as first line treatment for acute rejection. It has been shown by Theodorakis et al. that treatment for a first acute rejection episode with ATG reduces the number of recurrent rejections compared to treatment with methylprednisolone $(16 \% \text { vs. } 72 \%)^{2}$. In our study, the proportion of recurrent rejections was comparable (16 out of $131,12.2 \%$ ). Thus we probably reversed acute vascular rejection and prevented the occurrence of recurrent acute rejections.

After censoring for death with a functioning graft, graft half-life after year 1 turned out to be $\pm 12 \frac{1}{2}$ years. The extrapolated graft half-life was different between patients who underwent transplantation in the years 1983-1987, 1988-1991, and $1992-1995$ ( $\pm 11, \pm 13.5$, and \pm 19 years, respectively).

Graft loss after the first year was mainly related to the quality of function of the graft (creatinine clearance and proteinuria at year 1) and, to a lesser extent, to the age of the recipient. Renal function at year 1 has been reported to be one of the main prognostic parameters for graft survival ${ }^{23.25}$; proteinuria has also been reported to be a risk factor ${ }^{26.28}$.

Creatinine clearance is a reflection of the quantity of functioning nephrons and, thereby, of the quality of donor parameters (including age of donor) and insults placed upon the graft during procurement, preservation, and the first year after transplantation (DGF, cyclosporine nephrotoxicity, rejection). In this study, 25.4\% of the donors were over 50 years of age, while $7.8 \%$ were over 60 years. Also, in nearly half of them, cardiovascular diseases were the cause of death. Both high donor age and cardiovascular cause of death of the donor are related to a poorer kidney function ${ }^{29-31}$.

Proteinuria is a sign of structural damage of the nephron, mainly of the glomerulus. This damage can be pre-existent in the donor kidney, can be a result of the preservation and transplantation procedure, or can be due to factors occurring after transplantation, such as hypertension and chronic rejection. Some patients had residual function of their native kidneys or previous transplants. Although this residual urine production usually drops after the start of cyclosporine administration, it may be that native kidneys or a previous graft contribute to the 
proteinuria. Therefore, in our analysis, the influence of proteinuria on graft survival might have been underestimated.

Older recipients have a lower risk of graft failure. This is a confirmation of other reports $^{32.33}$ and is considered to be caused by a diminished immunological reaction with an increase in age and, thereby, a lower risk of chronic (immunological) rejection.

Mismatch for HLA-B was a risk factor for late graft failure when introduced as a single risk factor. This has been reported before ${ }^{44.35}$. Mismatch for HLA-B is also generally considered to be a risk factor for chronic rejection. However, in multivariate analysis, mismatch for HLA-B was no longer a risk factor for graft survival. This might have been due to the fact that mismatch for HLA-B is significantly correlated with creatinine clearance and does not contribute to graft survival when creatinine clearance is included in the model.

In our data, 0 HLA-A, - B, and -DR mismatched patients did not have a significantly better graft survival. This may have been due to the small number of patients in this data set and to the confounding 'noise' of other factors influencing graft function.

\section{Acute rejection}

Prevention of acute rejection is important. The vascular type of acute rejection, in particular, has been shown to be related to inferior graft survival ${ }^{21}$. Moreover, the need to increase the immunosuppressive load places the patient at an increased risk of infections and malignancies. The occurrence of acute rejection increases the duration of hospitalization. Patients at a higher immunological risk (high PRA in peak serum and/or third or fourth graft) were at a higher risk of rejection. These findings are a confirmation of previous findings $\mathrm{s}^{36,37}$.

An increase in CIT was related to a decreased risk of rejection. This was not due to the association between CIT and exchanged kidneys, resulting in a better HLA match. Our findings are at variance with reports that have shown that an increase in ischemia time induces inflammation and upregulation of HLA-molecules, thereby increasing the risk of rejection ${ }^{38}$. However, it has also been reported in rats that a temporary uremic state will decrease alloreactivity, resulting in less rejection of renal grafts ${ }^{39}$. Because CIT is related to DGF, our results may be the human counterpart of this 'uremic escape'.

HLA - B, or -DR mismatches result in a higher risk of rejection, but only in certain combinations. Compared to patients with 0 mismatches for HLA-A, -B, and -DR, patients with $\geq 1$ mismatch for either HLA-B or HLA-DR had an increased risk of acute rejection; however, patients with $\geq 1$ mismatch for HLA-A did not have an increased risk of rejection. In patients with $\geq 1$ mismatch for both HLA-A and $-B$, patients with mismatches for HLA-DR had a higher rejection rate than patients 
without mismatches for HLA-DR. Mismatches for HLA-B or -DR do not result in an increased risk of rejection in the other combinations, while HLA-A mismatches are related to a lower rejection rate in the $\geq 1 \mathrm{~B}$ and $0 \mathrm{DR}$ mismatch group. There is no evidence to support this last result in the literature, nor is there a biological explanation for it. Therefore, it may just be an artefact in our data set. Before conclusions can be drawn from this result, it must be confirmed in an independent data set. The influence of HLA mismatching is generally analyzed by adding up all mismatches for HLA-A, -B, and -DR. Transplants with 0 mismatches had a lower rejection rate than transplants with at least 1 mismatch for these $l o i^{40}$. Previous reports from our center, grouping mismatches in this way, confirmed these finding $s^{41 . A 2}$. Moreover, if the current data are analyzed in the way we did in our previous reports, the 0 HLA-A, -B, -DR mismatch group still had a significantly lower rejection rate $(20 \%)$ than patients with 1 mismatch for either HLA-A, -B, or -DR $(47 \%)$ and patients with $\geq 1$ mismatch for HLA-A, -B, and -DR (37\%; $P=0.01$ ). Using the technique of a hierarchical model-fitting, we were able to make some nuances to this general view. Specific groups at risk for (or protected from) rejection were discriminated by analyzing the interaction between mismatches for different HLA loci. Our study stressed the influence of the way in which data are grouped. The HLA system is very complicated, and not every mismatch seems to be as important as shown by the concept of 'taboo mismatches'43, 'acceptable mismatch program ${ }^{\prime 4,45}$, and 'non-inherited maternal antigens' ${ }^{\prime 4,46}$. Thus, by 'intelligent mismatching', it may be difficult to ascertain the beneficial effect of HLA matching on rejection rate in a single-center study. Moreover, the size of our study population may be too small to analyze all details of the influence of the complex HLA system on rejection.

\section{Need for dialysis after transplantation (DGF)}

DGF is an important complication after renal transplantation, contributing to lower graft survival and increased complexity of post-transplant care. We have found that parameters shown to be related to DGF are multifactorial, including immunological, preservation, and recipient parameters. Sensitization (\%PRA) has been reported to be related to DGF, possibly by inducing subclinical rejection ${ }^{47}$. Cold lymphocytotoxins, in particular, are reported to be detrimental to immediate graft function ${ }^{48}$. Ischemic/reperfusion damage (WITI and CIT) is well known to increase the DGF rate ${ }^{49}$. This is supported by the fact that grafts from living donors have a lower frequency of DGF than grafts from cadaveric donors ${ }^{50}$. Recipient parameters are also related to DGF. Type of pretransplant dialysis influenced the DGF rate. The DGF rate was higher in hemodialysis patients than in peritoneal dialyzed patients. This is in accordance with the results of van Loo et al. ${ }^{51}$, who showed that hemodialysis within 24 hours pretransplantation with bio-incompatible membrane and/or ultrafiltration increased the rate of early graft dysfunction. 
Generally, most of our hemodialysis patients were dialyzed with a bio-incompatible membrane (and ultrafiltrated) before transplantation. Complement and cytokines are activated by this and, thus, induce inflammation. It is difficult to estimate dry weight by clinical parameters ${ }^{52}$, and hemodialysis patients can be relatively dehydrated compared to CAPD patients. Dehydration after transplantation may increase vasoconstriction, resulting in DGF.

Size mismatch between graft and recipient has been reported to be related to an increased DGF rate ${ }^{33}$. Our finding that male recipients had a higher DGF rate supports this observation, because males generally have a higher body muscle mass and, thus, a need for more nephron mass. However, in our population, the combination of male recipient with female donor did not result in a higher risk of DGF (data not shown).

A poor score on the parameter 'general evaluation of the recipient' turned out to be a significant risk factor for DGF. Besides immunological selection (high PRA), this may also be related to a high prevalence of cardiovascular diseases in these patients. These patients did not tolerate aggressive hydration as well in the perioperative period, resulting in a decreased perfusion of the graft immediately after reperfusion. It has been shown that optimal intravascular filling with colloĩdal fluids, crystalloïds, and mannitol will reduce the frequency of $\mathrm{DGF}^{\mathrm{s}}$. In this study, neither mismatch for HLA nor type of preservation solution was significantly related to DGF.

\section{Duration of DGF}

The duration of DGF was mainly determined by the donor parameters 'gender' and 'age'. Both females and older persons ${ }^{55}$ are known to have a lower number of nephrons; therefore graft function has to recover to a higher extent before the recipient is dialysis-independent. We cannot rule out the possibility that other factors related to these parameters play a role (e.g., cause of death of donor) although these factors, when included into the tested parameters, did not turn out to be significantly related to duration of DGF by itself. Of interest is the finding that neither occurrence of rejection nor parameters related to the frequency of DGF were correlated to the duration of DGF.

\section{Duration of hospitalization}

In our study, duration of hospitalization was a result of both pretransplant conditions and post-transplant complications. The most important ones were the post-transplant complications 'DGF' and 'occurrence of acute rejection'. Of the pretransplant conditions, both 'age of donor' and 'age of recipient' were related to duration of hospitalization. The hospital stay increased with an increase in age. Older recipients had more morbidity. Age of donor was an independent risk factor. 
Immunized patients had a longer hospitalization, possibly due to postoperative complications, such as rejection and infections. Transplant period was related to duration of hospitalization. Patients who underwent transplantation in the first period (1983-1987) had a significantly shorter duration of hospitalization than patients in later periods. In recent years, more high-risk patients have been accepted for transplantation and the type of kidney donor has changed. The increase in high-risk patients is reflected in an increase in the percentage of patients with a score of 'poor' on the general evaluation, from $2.6 \%$ in the period 1983 1987 to $13.6 \%$ in the period $1992-1995(P=0.02)$. This unfavorable change in type of kidney donor is reflected in the increase in mean donor age between these periods from 34.7 years to 40.9 years $(P=0.02)$ and the decrease in the number of trauma donors from $56.8 \%$ to $36.6 \%$, while the number of donors dying due to subarachnoidal or intracranial bleeding increased from $12.8 \%$ to $29.0 \%(P=0.03)$. These changes will result in a longer duration of hospitalization.

\section{Creatinine clearance at 1 year after transplantation}

We confirm and extend the findings that renal function after transplantation has a strong impact on long-term graft survival ${ }^{6.23 .56}$. As function declines over the years, lower 1-year function will result in reaching the point of end-stage renal failure earlier. A decrease in renal function also increases the frequency and severity of hypertension and hyperlipidemia ${ }^{36}$. Both factors may have an impact on patient and graft survival ${ }^{57,5 \%}$. Factors related to creatinine clearance at year 1 can be grouped as donor/preservation-related (donor age, CIT, type of preservation), immunological (HLA-B mismatch), and recipient-related (recipient age, type of dialysis pretransplant, cause of renal failure). With an increase in age comes a decrease in the number of nephrons and renal function ${ }^{55.59}$. Older donors also have a higher incidence of chronic ischemic lesions. It has been shown by our group that a combination of these lesions with the use of cyclosporine results in worsened renal function ${ }^{\infty}$. The type of preservation solution used has an impact on renal graft function $^{61}$. However, it also reflects the period of transplantation (in the first period, only Eurocollins solution was used and in the latest period, UW and HTK are used in addition to Eurocollins) and the type of donor (currently, grafts from multiorgan donors (MOD) are preferentially preserved by UW, while HTK is used for NHB donors). Grafts from MODs are reported to function better than grafts from kidney-only donors ${ }^{2}$. As has been shown in animal studies, longer CIT may cause more ischemic/reperfusion damage ${ }^{63.64}$, resulting into fewer functional nephrons and a lower creatinine clearance. Longer duration of hospitalization is a parameter that may reflect a possible complicated postoperative course (especially DGF and acute rejection). This may explain the association of duration of hospitalization with a poorer renal function at 1 year. Mismatch for HLA-B is related to a lower creatinine clearance. This is most likely an early sign of chronic 
rejection, because mismatch for HLA-B has been reported to be associated with lower graft survival ${ }^{34,15}$. In this study, creatinine clearance was one of the major factors influencing long-term graft survival. In the analysis of graft survival, mismatch for HLA-B was a risk factor for graft failure when introduced as a single factor; however, when creatinine clearance was introduced into the model, it was no longer a significant risk factor. Recipient factors are also related to renal function. Increasing recipient age results in a poorer renal function. This is not due to selection of older or non-trauma donors for older recipients. An unproven explanation may be a decreased perfusion of the graft by lower cardiac output or more frequent use of $\mathrm{ACE}$ inhibitors for the treatment of hypertension. The significantly better renal function for patients with type 2 diabetes mellitus may reflect hyperfiltration or a systemic error in the calculation of the creatinine clearance by the Cockcroft-Gault formula because of an altered relationship between weight and muscle mass in these patients. Also, transplantation of these patients while they still have a residual renal function of their native kidneys (e.g., because of shorter duration of dialysis pretransplant) may contribute to a higher creatinine clearance. CAPD pretransplantation is related to a higher renal function at year 1. Residual function of CAPD patients, which is known to be preserved longer than in $\mathrm{HD}$ patients ${ }^{65}$, may add to graft function resulting in a higher creatinine clearance. However, it is known that residual function decreases when patients use cyclosporine, thereby making this possibility unlikely. Also, the higher weight $(+2.4 \mathrm{~kg})$ and younger age of CAPD patients $(-2.4 \mathrm{yr})$ compared to HD patients does not explain the difference in creatinine clearance. It is unlikely that the type of pretransplant dialysis structurally influences the graft after transplantation. Thus, differences in graft function must be functional. Possible scenarios for functional changes in graft function are: 'steal effect' of the persistent dialysis shunt in HD patients, resulting in lower perfusion of the graft, and the recruitment of renal functional reserve by high-protein intake in former CAPD patients. A high-protein intake results in a higher glomerular filtration rate, also in renal allografts ${ }^{66,67}$. At the time of transplantation, our HD patients had a proteinrestricted diet, in contrast to CAPD patients. It is plausible that after transplantation these differences in diet are still present.

In this single center study, we analyzed the impact of perioperative parameters on the clinical outcome of renal cadaveric transplantation using multivariate techniques. The results show that several donor, preservation, and recipient parameters influence short- and long-term outcome of the renal allograft.

The influence of histocompatibility parameters was modest. Only in rejection rate and creatinine clearance at year 1 did matching for some HLA loci result in a better outcome. However, the analysis of the influence of HLA mismatching on clinical outcome in this study may have been hampered by its complexity, 
'intelligent mismatching', and the relatively small sample size. Not only HLA matching, but also the presence of cytotoxic HLA antibodies and previous transplants influenced DGF and short-term patient and graft survival. Parameters other than histocompatibility are related to short- and long-term outcome parameters. These parameters are donor, preservation, and recipient-related. Some of these factors may be influenced by other donor and recipient management and may therefore be potential targets for improvement of the renal transplant outcome. This is especially true for the prevention of DGF by reducing CIT (e.g., improve in-hospital logistics by reducing waiting time for operation theatre) and modifying pretransplant hemodialysis (use of biocompatible membranes and prevention of ultrafiltrating large volumes). Other factors are less easily influenced (e.g., donor age, recipient age, NHB donor, general evaluation of recipient). Yet, it is important to know the relevant factors because donor and patient characteristics are changing to unfavorable types. By selecting an optimal donor-recipient combination, the accumulation of unfavorable parameters may be prevented, thus improving the clinical outcome of renal cadaveric transplantation. 


\section{References}

1. Forsyth JLR. In Forsythe JRL (ed): Transplantation surgery. London, Philadelphia, Toronto, Sydney, Tokyo: WB Saunders Company Ltd; 1997, Chapter 6 Kidney transplantation.

2. Statistisch verslag van de Registratie Nierfunctievervanging Nederland (Renine) 1998. Eds Ramsteijn PG, de Charro FTh, Geerlings W, Krediet RT, Tjandra YI. Utrecht, Elinkwijk, p 22.

3. Christiaans MHL, van Hooff JP, Nieman F, van den Berg-Loonen EM. HLA-DR matched transfusions; development of donor-specific T- and B-cell antibodies and renal allograft outcome. Transplantation 1999; 67: 1029-35.

4. Cockcroft DW, Gault MH. Prediction of creatinine clearance from serum creatinine. Nephron 1976, 16: 31-41.

5. Schouten JA. In IA Schouten (eds): Een praktische inleiding in methodologie en analyse. Houten Diegem: Bohn Stafleu van Lochum; 1999, 2e editie 1999.

6. Vanrenthergem Y, Peeters J: In Touraine JL, et al (eds): Late graft loss. Great Brittain: Kluwer Academic Publishers; 1997: 85.

7. Cole E, Naimark D, Aprile M, Wade J, Cattran D, Pei Y, Fenton S, Robinette M, Zaltsman J, Bear R, et al. An analysis of predictors of long-term cadaveric renal allograft survival. Clin Transplant 1995; 9: 282-8.

8. Diethelm AG, Deierhoi MH, Hudson SL, Laskow DA, Julian BA, Gaston RS, Bynon JS, Curtis JJ. Progress in renal transplantation. A single center study of 3359 patients over 25 years. Ann. Surg. 1995; 221: 446-57.

9. Smits JM, de Meester J, Persijn GG, Claas FH, Vanrenthergem Y. Long-term results of solid organ transplantation. Report from the Eurotransplant International Foundation. Clin Transpl 1996: 109-27.

10. Thorogood J, van Houwelingen JC, van Rood JJ, Zantvoort FA, Schreuder GMTh, Persijn GG. Factors contributing to the long-term kidney graft survival in Eurotransplant. Transplantation 1992; 54: 152-8.

11. Cecka JM, Terasaki PI. The UNOS scientific renal transplant registry. United Network for Organ Sharing. In: Terasaki PI, Cecka JM (eds): Clinical Transplantation 1995. Los Angeles, UCLA Tissue Typing Laboratory, 1996: 1-18.

12. Opelz G. The benefit of exchanging donor kidneys among transplant centers. $\mathrm{N}$ Engl J Med 1988; 318: 1289-92.

13. Opelz G. Collaborative Transplant Study. 10 year report. Transplant Proc 1992; 24 : 2342-55.

14. Opelz G, Mytilineos J, Wujciak T, Schwarz V, Back D. Current status of HLA matching in renal transplantation. The Collaborative Transplant Study. Clin Investig 1992: 70: 767-72.

15. Thorogood J. Houwelingen JC van , Rood JJ van, Persijn GG. The impact of centre variation on the HLA-DR matching effect in kidney graft surival. Transplantation 1989; 48: 231-7. 
16. Raine AEG, Margreiter R, Brunner FP, Ehrich JHH, Geerlings W, Landais P, Loirat C, Mallick NP, Selwood NH, Tufveson G, Valderrabano F. Report on management of renal failure in Europe, XXII, 1991. Nephrol Dial Transplant 1992; 7 (Suppl. 2): 7-35.

17. Disney AP. Demography and survival of patients receiving treatment for chronic renal failure from the Australia and New Zealand Dialysis and Transplant Registry. Am J Kidney Dis 1995; 25: 165-75.

18. Wijnen RMH, Booster MH, Stubenitsky BM, de Boer J, Heineman E, Kootstra G. Outcome of transplantation of non-heart-beating donor kidneys. Lancet 1995; 345: 1067-70.

19. Cho YW, Terasaki PI, Cecka JM, Gjertson DW. Transplantation of kidneys from donors whose hearts have stopped beating. NEJM 1998; 338: 221-5.

20. Almond PS, Matas AJ. Gillingham KJ, et al. Risk factors for chronic rejection in renal allograft recipients. Transplantation 1993; 55: 752-6.

21. van Saase JLCM, van der Woude FJ, Thorogood J, et al. The relation between acute vascular and interstitial renal allograft rejection and subsequent chronic rejection. Transplantation 1995; 59: 1280-5.

22. Theodorakis J, Schneeberger H, Illner WD, Stangl M, Zanker B, Land W. Aggressive treatment of the first acute rejection episode using first-line antilymphotic preparation reduces further acute rejection episodes after human kidney transplantation. Transpl Int 1998; II S1: S86-S9.

23. Kuo PC, Johnson LB, Schweitzer EJ, Alfrey EJ, Waskerwitz J,Bartlett ST. Utilization of the older donor for renal transplantation. Am J Surg 1996; 172: 551-7.

24. Kasiske BL. Clinical correlates to chronic renal allograft rejection. Kidney Int 1997. 52 (suppl 63): S71-S4.

25. Terasaki PI, Gjertson DW, Cecka JM, Takemoto S. Fit and match hypothesis for kidney transplantation. Transplantation 1996; 62: 441-5.

26. Brenner BM, Cohen RA. Milford EL. In renal transplantation, one size may not fit all. J Am Soc Nephrol 1992; 3: 162-9.

27. Terasaki PI, Koyama H, Cecka JM, Gjertson DW. The hyperfiltration hypothesis in human renal transplantation. Transplantation 1994; 57: 1450-4.

28. Alcalde G, Esallada R, Rodrigo E, Cotorruelo JG, Zubimendi JA, de Francisco E. Arias M. Disproportion between kidney graft and recipient size is the main predictor of long-term proteinuria. Transplant Proc 1997; 29: 127-8.

29. Cecka JM. Donor factors. In: Terasaki PI (eds), Clinical Transplantation 1987. Los Angeles, UCLA Tissue Typing Laboratory, 1988: 423-34.

30. Cho YW, Terasaki PI. Impact of new variables reported to the UNOS registry. In: Terasaki PI, Cecka JM (eds), Clinical Transplantation 1997. Los Angeles, UCLA Tissue Typing Laboratory, 1998: 305-14.

31. Nickerson P. Jeffery J, Gough J. McKenna R, Grimm P, Cheang M, Rush D. Identification of clinical and histopathologic risk factors for dimishe renal function 2 years posttransplant. J Am Soc Nephrol 1998: 9: 482-7. 
32. Jassal SV, Opelz G, Cole E. Transplantation in the elderly: a review. Geriatr Nephrol Urol. 1997; 7: 157-65.

33. Roodnat J, Zietse R, Mulder PG, Rischen-Vos J, van Gelder T, Uzermans JN, Weimar W. The vanishing importance of age in renal transplantation. Transplantation 1999; $67: 567-80$.

34. van Hooff JP, van der Steen GJ, Schippers HMA, van Rood JJ. Efficacy of HL-A matching in Eurotransplant. Lancet 1972; ii: 1385-8.

35. Thorogood J, Persijn GG, Schreuder GMTh, D'Amaro J, Zantvoort FA, van Houwelingen JC, van Rood JJ. The effect of HLA matching on kidney graft survival in separate posttransplantation intervals. Transplantation 1990; 50: 146-50.

36. Stratta RJ, Oh CS, Sollinger HW, Pirsch JD, Kalayoglu M, Belzer FO. Kidney retransplantation in the cyclosporine era. Transplantation 1988; 45: 40-5.

37. Sanfilippo F, Vaughn WK, LeFor WM, Spees EK. Multivariate analysis of risk factors in cadaver dono kidney transplantation. Transplantation 1986; 42: 28-34.

38. Goes N, Urmson J, Ramassar V, Halloran PF. Ischemic acute tubular necrosis induces an extensive local cytokine response: evidence for induction of interferongamma, transforming growth factor-beta 1, granulocyte-macrophage colonystimulating factor, interleukin-2, and interleukin-10. Transplantation 1995, 59; $565-72$.

39. van Schilfgaarde R. In: Uremic escape of renal allograft rejection. A passenger leukocyte mediated phenomenon studied in the rat. Thesis. Tongeren, Michiels 1978.

40. Washburn WK, Shaffer D, Conway P, Madras PN, Monaco AP. A single-center experience with six-antigen-matched kidney transplants. Arch Surg 1995; 130: 277-82.

41. Christiaans MHL, Peltenburg HG, Niemann FHM, Kootstra G, Lavrijssen ATJ, van den Berg-Loonen PM, Tiebosch A, Leunissen KML, van Hooff JP. The impact of ischaemic lesions in the donor kidney, donor age, recipient age and HLA (A, B, C, DR, DQ) matching on clinical course after kidney grafting. Transpl Int 1992; 5 (S1): S627-S8.

42. Christiaans MHL, van den Berg-Loonen PM, Peltenburg HG, Leunissen KML, van Hooff JP. More favourable clinical course in kidney allograft recipients due to HLA-B+ DR matching. Transplant Proc 1991; 23: 2674.

43. Doxiadis II, Smits JM, Schreuder GM, Persijn GG, van Houwelingen HC, van Rood J, Claas FH. Association between specific HLA combinations and probability of kidney allograft loss: the taboo concept. Lancet 1996; 348: 850-3.

44. Claas FH, van Leeuwen A, van Rood JJ. Hyperimmunized patients do not need to wait for an HLA identical donor. Tissue Antigens 1989; 34: 23-9.

45. Claas FH. Negative cross-match selection of HLA-DR compatible kidneys for highly sensitised patients. Immunol Lett. 1991; 29; 61-4

46. Smits JM, Claas FH, van Houwelingen HC, Persijn GG. Do noninherited maternal antigens (NIMA) enhance renal graft survival? Transpl Int 1998; 11: 82-8. 
47. Baldwinn WM, Halloran PF. Clinical syndromes associated with antibody in allografts. In: Kidney transplant rejection (3rd ed), edited by Racusen LC, Solez K, Burdick J, New York Basel Hong Kong, Marcel Dekker Inc, 1998: 134.

48. Lobo PI, Westervelt FB, White C, Rudolf LE. Cold lymphocytotoxins: an important cause of acute tubular necrosis occurring immediately after transplantation. Lancet 1980; 2: 879-82.

49. Shoskes DA, Halloran PF. Delayed graft function in renal transplantation: etiology, management and long-term significance. J Urol 1996; 155: 1831.

50. Terasaki PI, Cecka JM, Gjertson DW, Takemoto S. High survival rates of kidney transplants from spousal and living unrelated donors. NEJM 1995; 333: 333-6.

51. van Loo AA, Vanholder RC, Bernaert PR, Vermassen FE, van der Vennet M, Lameire NH. Pretransplantation hemodialysis strategy influences early renal graft function. J Am Soc Nephrol 1998; 9: 473-81.

52. Leunissen KML, Kouw P, Kooman JP, Cheriex EC, de Vries PMJM, Donker AJM, van Hooff JP. New techniques to determine fluid status in hemodialyzed patients. Kidney Int 1993; 43: S50-S6.

53. Senel FM, Karakayali H, Moray G, Haberal M. Delayed graft function: predictive factors and impact on outcome in living-related kidney transplantations. Renal Fail. 1998; 20: 589-95.

54. Tiggeler RG, Berden JH, Hoitsma AJ, Koene RA. Prevention of acute tubular necrosis in cadaveric kidney transplantation by the combined use of mannitol and moderate hydration. Ann. Surg 1985: 201: 246-51.

55. McLachlan M: In Macias Nunez JF, Cameron JS (eds): Renal function and disease in the elderly. Chapter 1 Anatomical, structural and vascular changes in the aging kidney. London, Durban, Singapore, Sydney, Toronto, Wellington: Butterworths; 1987: 12-3. Ist edition.

56. Kasiske BL, Umen AJ. Persistent hyperlipidemia in renal transplant patients. Medicine (Baltimor) 1987; 66: 309-16.

57. Opelz G, Wujciak T, Ritz E. Association of chronic kidney graft failure with recipient blood pressure. Kidney Int 1998; 53: 217-22.

58. Braun WE. Long-term complications of renal transplantation. Kidney Int 1990; 37: 1363-78.

59. Gaber LW, Moore LW, Alloway RR, Amiri MH, Vera SR, Gaber AO. Glomerulosclerosis as a determinant of posttransplant function of older donor renal allografts. Transplantation 1995; 60: 334-9.

60. Leunissen KML, Bosman FT, Nieman FHM, Kootstra G, Vromen MAM, Noordzij TC, van Hooff JP. Amplification of the nephrotoxic effect of cyclosporine by preexistent chronic histological lesions in the kidney. Transplantation 1989; 48: 590-3.

61. Ploeg RJ, van Bockel JH, Langendijk PTH, Groenewegen M, van der Woude FJ, Persijn GG, Thorogood J, Hermans J. Effect of preservation solution on results of cadaveric kidney transplantation. Lancet 1992; 340: 129-36. 
62. Smits JM, De Meester J, Persijn GG, Claas FH, van Houwelingen HC. The outcome of kidney grafts from multiorgan donors and kidney only donors. Transplantation 1996; 62: 767-71.

63. Wanders A, Akyürek ML, Waltenberger J, Ren ZP, Stafberg C, Funa K, Larsson E, Fellstrōm B. Ischemia-induce transplant arteriosclerosis in the rat. Arterioscler Thromb Vasc Biol 1995: 15: 144-55.

64. Tullius SG, Heemann U, Hancock WW, Azuma H, Tilney NL. Long-term kidney isografts develop functional and morphological changes that mimic those of chronic allograft rejection. Ann Surg 1994; 220: 425-35.

65. Lysaght MJ, Vonesh EF, Gotch F, Ibels L, Keen M, Lindholm B, Nolph KD, Pollock CA, Prowant B, Farrell PC. The influence of dialysis treatment modality on the decline of remaining renal function. ASAIO Trans 1991; 37: 598-604.

66. ter Wee PM, Tegzess AM, Donker AJ. Pair-tested renal reserve filtration capacity in kidney recipients and their donors. J Am Soc Nephrol 1994; 4: 1798-808.

67. Ader JL, Tack I, Durand D, Tran Van T, Rostaing L, Suc JM. Renal functional reserve in kidney and heart transplant recipients. J Am Soc Nephrol 1996; 7: $1145-52$. 


\section{Chapter 9}

\section{General discussion}




\section{General discussion}

Renal transplantation evolved from an experimental treatment with a high morbidity for a limited number of highly selected patients in the 1960s to a preferred treatment for most patients with end-stage renal failure by the end of the 1980 s.

From 1980 to the mid-1990s, the pretransplant work-up, surgical procedure, and follow-up were quite uniform in Western Europe. Many centers were transfusing patients before placing them on the renal transplant waiting list. Screening for antiHLA antibodies and the pretransplant crossmatch were done with complementdependent cytotoxicity assays. Kidneys were allocated by organ exchange organizations such as Eurotransplant. HLA matching between donor and recipient was the most important parameter for allocation. Most transplant recipients were given a cyclosporine-based immunosuppressive regimen. Patient and graft survival were satisfactory and the morbidity of the procedure was considerably lower than it had been in the early 1970 s.

In the 1990s, some changes occurred. Other screening and crossmatch techniques were introduced. The beneficial effect of blood transfusions was called into question, and it was hypothesized that the beneficial effect was dependent on the HLA-DR match between blood transfusion donor and recipient. Tacrolimus and mycophenolate mofetil were registered as new immunosuppressive agents.

Because of the good patient and graft survival, the criteria for placing patients on the waiting list have now become less strict leading to an increase in potential recipients. These are generally older recipients and recipients with more comorbidity. The increase in the number of potential recipients has not been accompanied by a parallel increase in the number of donors, and so the donor shortage has increased considerably. As a result, kidneys are currently being accepted for transplantation that would have been refused in the 1970s. Cadaveric donors have become older and the cause of death has changed from trauma to cerebrovascular diseases. Moreover, the use of living (unrelated) donors is being encouraged.

After a successful transplantation, there is an increase in the quality of life and in life expectancy ${ }^{2}$. Thus, some of the goals of renal transplantation have been reached. However, by 10 years post-transplantation, only about half of all recipients are still alive with a functioning graft. Many grafts are lost due to chronic transplant dysfunction. Both immunological and non-immunological factors may play a major role in this. In the next sections, the consequences of the recent changes in the transplant protocol on acute rejection incidence and graft survival will be discussed. 


\section{Blood transfusion}

In the azathioprine era, it was proven that patients who received a blood transfusion had about a $20 \%$ better graft survival than those who did not ${ }^{3,4}$. This effect occurs as early as after one transfusion. ${ }^{4.5}$. Many questions about this effect have not been answered, such as the quality of transfused blood, the mechanism behind the transfusion effect, etc. In the cyclosporine era, the question was raised as to whether this beneficial effect still existed.

Many centers have abandoned the policy of deliberately transfusing patients on the renal transplant waiting list. However, Opelz et al., in a randomized prospective trial, have reconfirmed a beneficial effect of transfusion on graft survival in patients treated with cyclosporine. Patients who received three transfusions have a $9 \%$ better 5-year graft survival rate than patients who received no transfusion (79\% vs. $70 \%$, respectively) ${ }^{6}$. Although the beneficial effect of transfusions has become smaller, this effect is still of clinical importance.

It has been reported that matching for HLA-DR between transfusion donor and recipient results in a lower grade of panel reactive antibodies ${ }^{7,8}$, a lower rejection rate $^{k .9}$, and better graft survival in recipients of a renal ${ }^{7}$ or heart transplant ${ }^{10}$. These results have been supported by in vitro data. In one study, HLA-DR-shared transfusions resulted in a decreased frequency of cytotoxic T-cell precursors ${ }^{11}$; however questions have since been raised about this study ${ }^{12,13}$.

Studies on the beneficial effect of DR matching for transfusion donor and recipient have some shortcomings. The occurrence of anti-HLA antibodies was detected by testing sera against a panel of third-party lymphocytes and not against the transfusion donor itself. Also, reactivity of these antibodies was usually tested only against $\mathrm{T}$ cells and not against $\mathrm{B}$ cells. The sensitization grade in the study by Lagaaij et al. was rather high in the nonmatched group?. These authors, the only ones who reported a significant difference in renal graft survival, studied only patients treated with azathioprine and not with cyclosporine. In most studies, patients and controls were transfused with different numbers of units of blood and controls were transfused with random blood instead of nonmatched blood. Also, the number of patients in many studies was rather small.

For these reasons, we analyzed a large group of patients $(n=247)$ on the renal transplant waiting list for the presence of donor-directed antibodies (for both class I and II) after transfusion of one unit of DR-matched or -nonmatched leukocyte-poor erythrocyte concentrate (Chapter 2). In contrast to Lagaaij et al., we found that the frequency of donor-directed antibodies (for both class I and II) after transfusion was low and did not differ between recipients of DR-matched and -nonmatched transfusion. Furthermore, rejection rate and graft survival after transplantation did not differ between the two groups in multivariate analysis. We postulated that the effect of DR matching in previous studies was due to methodological shortcomings 
(different number of transfusions between controls and patients) and a better HLA-DR match between recipient and kidney donor in the Lagaaij et al. study. At the time of their study, allocation of kidneys was based on HLA-B match. Patients with an HLA-DR-matched transfusion most commonly had a frequent DR antigen and, thus, a high chance of receiving a HLA-DR matched kidney due to linkage disequilibrium between HLA-B and HLA-DR antigens. Their results may, therefore, be positively influenced by the indirect beneficial effect of HLA-DR matching between kidney donor and recipient. We have performed a multivariate analysis to control for such confounders.

The results of this study do not support the recommendation to match blood donor and recipient for HLA-DR. Transfusion according to our protocol results in a low percentage of patients with donor-directed antibodies. Because the beneficial effect of transfusion still exists in the cyclosporine era, we have no reason to change our protocol of transfusing one random unit of blood to patients before they are placed on the waiting list.

\section{Screening}

Screening for anti-HLA antibodies is part of the pretransplant work-up. In this way anti-HLA antibodies are detected.

Antibody titer may vary over time and screening has to be performed regularly. At our center, every 3 months serum of patients on the renal transplant waiting list is screened; when necessary, screening is performed more frequently (e.g., after transfusion). Both the percentage of panel reactive antibodies (\% PRA) and the HLA specificity of the antibodies are recorded. Pretransplant detection of these antibodies is important because the presence of donor-directed HLA class 1 antibodies in current serum is an absolute contraindication for transplantation. The importance of the presence of class II antibodies in current serum and/or the presence of antibodies (class I and II) in peak serum has still not been established, although an increased incidence of rejection and graft loss have been reported.

In our center, the presence of antibodies with specificity against donor antigens, either class I or II, in any pretransplant serum is a contraindication for transplantation. Patients with a high \%PRA have a high risk of rejection and will generally receive additional immunosuppression.

The standard screening technique used is a complement-dependent lymphocytotoxicity (CDC) assay. It can be performed for HLA class I antibodies (NIH) and for HLA class II antibodies (TCF). CDC has a number of disadvantages, namely, the need for a panel of viable lymphocytes, the fact that it is timeconsuming, and the large intercenter variability.

The number of patients on the waiting list is increasing because of the organ shortage. The screening of many sera with the standard CDC assays is a great deal of work for the tissue-typing laboratory. To reduce the workload, newer screening 
procedures are being sought and newer screening techniques introduced. Such techniques are flow cytometry and enzyme-linked immunosorbant assay (ELISA).

Flow cytometry (FC) screening uses a pool of different lymphocytes. Sera without PRA that need no further investigation can be quickly discriminated from sera that must be investigated further to determine \%PRA and HLA specificity. Flow cytometry is dependent on the availability of large numbers of viable cells and, therefore, is not suitable for routinely screening sera for HLA specificity and \%PRA. Further investigation of FC-positive sera has to be performed using other techniques.

Another method that can be used for selecting antibody-positive sera for further screening is Quikscreen, an ELISA assay using beads covered with soluble HLA (sHLA) antigens to detect anti-HLA antibodies. sHLA antigens play a role in screening sera to determine \%PRA and HLA specificity in the commercial ELISA kit Prastat. Compared to CDC screening. Prastat has the advantages of standardization, low intercenter variation, and HLA-IgG specificity. However, the number of antigens presented is relatively low, and many antigens are represented only once or twice in the panel. The kit is expensive and the clinical significance of different results between ELISA and CDC is still not yet clear. The manufacturer explicitly states in the instruction manual that the clinical significance of \%PRA or HLA specificity based on Prastat has not yet been demonstrated. Although originally presented as HLA class I-specific, it has become clear that both class I and II antibodies are detected.

ELISA has been compared extensively for screening HLA class I antibodies with CDC, most frequently the NIH technique. However, because ELISA detects both class I and II antibodies, it needs to be compared with CDC screening techniques that also detect HLA class I and II antibodies. In Chapter 3, we compared ELISA (Prastat) and CDC (class I and II) for the detection of anti-HLA antibodies in sera of renal transplant recipients. We found that the method agreement between ELISA and NIH was insufficient to replace one method by the other. The method agreement between ELISA and TCF was even worse.

Furthermore, the relationship between screening result and graft survival and rejection was assessed. None of the assays was related to graft survival. The comparison of ELISA and NIH using pretransplant sera showed that the results of both assays equally discriminated patients at risk for rejection, both in univariate analysis and in multivariate analysis, while the result of TCF was not related to rejection. With regard to post-transplant sera and change in antibody status from pretransplant to post-transplant, CDC correlated better with rejection than ELISA. This held true for both NIH and TCF.

The patients in the study underwent transplantation after the presence of donordirected HLA antibodies in any pretransplant serum was excluded by CDC. The question of whether detection of donor-directed HLA specificity pretransplantation 
contributes to the risk of rejection can only be answered for ELISA.

Compared to ELISA-negative sera, only sera with antibody specificity, donordirected or non-donor-directed, were a risk factor for rejection. ELISA-positive sera without attributed HLA specificity were not a risk factor for rejection.

For NIH, \%PRA, and not antibody specificity, was a risk factor for rejection. It can be concluded from this study that ELISA and NIH provide additional information on sensitization. The real place of ELISA compared to CDC has yet to be determined. Currently, it seems that the costs of the ELISA kit are a drawback. ELISA cannot replace the CDC technique in screening for less frequent antibodies and crossmatching. The latter may be resolved if the ELISA-based crossmatch assay is introduced. Such an assay, Crossstat, is currently under investigation.

In comparing ELISA and CDC, one has to consider the fact that the patients have been selected by CDC assays. The best way to prove the clinical value of ELISA compared to CDC would be in a trial in which patients are randomized to either ELISA or CDC for screening and crossmatching and in which both groups are compared for occurrence of post-transplant outcome. Until results of such trial are known, factors other than efficacy, such as costs, will determine which tests are used.

\section{Crossmatch}

When selecting a recipient for a cadaveric donor, the patient's immunological profile is taken into account. If a donor-recipient combination is selected, the final test to be performed is the crossmatch between donor cells and recipient serum. The standard crossmatch is based on the CDC technique. A positive T-cell CDC crossmatch is an absolute contraindication for proceeding with transplantation, while a positive B-cell CDC crossmatch without donor-directed class II antibodies in the screening is not.

Highly immunized patients often react with a positive crossmatch and, consequently, are seldom given a transplant. For this reason, a special program has been developed, the Highly Immunized Trial (HIT) by Opelz ${ }^{14}$. In the HIT program, a tray with sera of highly immunized patients is sent to all participating centers and is crossmatched with every donor. In the case of a negative crossmatch with serum of a patient on the tray, the kidney is transplanted into this patient.

A new crossmatch technique based on the technique of flow cytometry (FC) has been reported. The FC crossmatch is more sensitive and T-cell IgG-specific. In Chapter 4, a review of the literature on FC crossmatching is given, and the validation of the FC crossmatch, as performed in our center, is described.

Several centers have reported FC crossmatch to be superior to NIH crossmatch $^{15-20}$, although others did not find such a difference when anti-human globulin CDC was 
used as the crossmatch technique ${ }^{17}$. We analyzed the additive value of pretransplant FC crossmatching in CDC-negative patients (Chapter 5). No difference was found in the 1-year graft survival or in the acute rejection rate between patients positive or negative by FC. In contrast, when FC crossmatch had been the crossmatch technique, $28 \%$ of our recipients would have been denied transplantation with their donor kidney, while in fact they were doing well. This suggests that the FC crossmatch may be too sensitive. This conclusion is supported by the fact that no report has been published of a hyperacute rejection in a renal allograft recipient transplanted with a $\mathrm{CDC}$-negative/FC-positive pretransplant crossmatch. From a clinical point of view, these positive FC results should be considered as falsepositive results. We have hypothesized that our approach to pretransplant screening prevents transplantation of patients with clinically significant antibodies. Donors are excluded for a patient if any serum of this patient has or had in the past shown anti-HLA antibodies with specificities directed against this donor. We conclude that the FC crossmatch has no additional effect in immunologically well-documented patients transplanted with a negative pretransplant CDC crossmatch.

\section{Post-transplant immunological monitoring}

It is no routine procedure to test sera post-transplantation for the presence of antibodies. However, such immunological monitoring is important. It has been shown that detection of anti-HLA cytotoxic antibodies after transplantation is related to rejection, both when found in a screening assay ${ }^{21.22}$ or by donor-specific crossmatch $^{23,24}$. In Chapter 6, we compared post-transplant crossmatches by CDC and FC in our population. Patients who became positive by either CDC or FC were at an increased risk of rejection and graft loss. However, the increased in vitro sensitivity of the FC crossmatch was not reflected in a comparable increase in the number of patients at risk. This might be due to the definition of FC positivity. Discrimination between negative and positive results was based on the mean reactions of a number of 'negative' human control sera. These negative sera show variability in their reactivity against lymphocytes from different individuals. The cutoff point, defined by the mean channel plus three standard deviations of negative control sera, might result in a lack of detection of low but significant antibody concentrations. To avoid the use of a negative control serum in the definition of the cutoff point, the FC activity of sera before and after transplantation was compared directly. Since both sera were tested with the same target cells, this comparison was considered justified. When the patients were grouped into quartiles according to their relative change in fluorescence ratio, patients in the quartile with the greatest relative increase in fluorescence ratio were shown to be at risk for rejection and graft loss. Interestingly, patients in the quartile with the greatest decrease in fluorescence ratio did best. Whether this might be due to $\lg A$ antibodies is currently under investigation. Our results may be used to discern in an 
early stage after transplantation a large group of patients at risk for graft loss. This group might potentially benefit from an adjustment in their immunosuppressive regimen.

\section{Matching for HLA antigens}

Registry studies consistently show the importance of HLA matching in both rejection rate and graft survival. Minimizing HLA mismatches is an important criterion for allocation. Traditionally, the number of mismatches for the HLA-loci $\mathrm{A}, \mathrm{B}$, and DR are counted from 0 to 6 . With an increase in the number of mismatches, rejection rate increases and graft survival decreases. There is discussion about the level of HLA typing needed for HLA matching. Increasing the typing level to a more detailed level, as well as decreasing the typing level to a more general level have been advocated.

With improved methods of detection, some HLA antigens, supposed to represent only one antigen, could in fact be split into several different antigens by serology or by DNA typing. The original antigen is called the broad antigen. Improvement in graft survival has been shown both for matching HLA-A and -B antigens at the serologically split level ${ }^{25}$ and for matching for HLA-DR at the DNA level ${ }^{26}$ compared to matching for broad antigens. However, by introducing matching on the split or DNA level, the number of HLA antigens increases, resulting in a lower percentage of well-matched donor-recipient combinations.

On the other hand, different broad HLA-A and -B antigens have been shown to share amino acid residues with minimal or no sequence variability. Because these different residues share antigenic determinants, they constitute a basis for common groups, also called crossreactive groups (CREGs) ${ }^{27}$. Many of the antibodies formed are directed against these CREGs and some consider it to be sufficient to match only for CREGs. The concept of CREGs was studied especially in the USA, although in the Eurotransplant region a low chronic rejection rate due to matching for broadly reacting antigens has also been reported ${ }^{28}$. The advantage of matching for CREGs is the possibility of providing more patients with a well-matched kidney. It has been reported, also by our group, that matching for HLA-DQ results in a low rejection rate comparable to that when matching for HLA-DR ${ }^{29.30}$. Because the HLA-DQ locus is less polymorphic than the HLA-DR locus, matching for HLA-DQ instead of for HLA-DR will result in transplantation of more matched grafts. However, the HLA-DR and HLA-DQ loci have a strong linkage disequilibrium and the patients were prospectively matched for HLA-DR antigens. Moreover, the number of patients in the studies was relatively small. Therefore, the question of whether matching for HLA-DQ is preferable to matching for HLA-DR cannot be answered yet. This question can only be answered in a randomized study in which patients are prospectively matched for either HLA-DR or HLA-DQ. 
To decide as to at which level of HLA typing matching has to be performed is, among other factors, dependent on the size of the recipient pool. It is preferable to provide the best match (= matched on split or DNA level); however, if no wellmatched donor-recipient combination is available, e.g., in the case of a small recipient pool, matching for CREGs is probably better than random allocation.

The concept of counting the number of mismatches has disadvantages. Implicitly, every mismatch is considered of equal importance. Yet, it has been shown that this is not the case. The immunogenicity of different HLA antigens may vary and the time period that a mismatch has influence on clinical outcome varies for the different HLA loci.

Recently, the concept of 'taboo' mismatches has been introduced ${ }^{31}$. Analysis of Eurotransplant data showed that not every mismatch results in early graft loss. Mismatches that do result into poorer graft survival are called 'taboo' and those that do not are named 'indifferent'. These taboo mismatches are not recipientspecific, but are intrinsic to the immunogenicity of specific combinations of antigens.

Also, in the concept of Acceptable Mismatch ${ }^{32,33}$. HLA matching for donorrecipient combinations does not give the same value to every mismatch. For patients with a high \%PRA, it is very difficult to provide a good match with a negative crossmatch. They rarely undergo transplantation and their waiting time is very long. In the Acceptable Mismatch program, HLA class I antigens are selected against which the patient has not formed antibodies. Therefore, a number of mismatches are now acceptable and matching has become feasible. In the Eurotransplant allocation scheme, patients on the Acceptable Mismatch program, have preference over other patients if a donor with a suitable HLA typing is available.

It has been reported that HLA-DR mismatch is important in the early phase after transplantation (up to 6 months), while for a graft that has survived this period the number of HLA-DR mismatches does not matter anymore ${ }^{3.35}$. From 6 months up to 3-5 years the number of HLA-B mismatches is of importance, while the number of HLA-A mismatches probably matters for grafts surviving for a longer period of time. Therefore, simply adding the number of mismatches does not seem to be the best way to allocate kidneys.

The allocation of grafts by Eurotransplant has recently been changed, replaced by an algorithm originally designed by Wujciak and Opelz ${ }^{36}$. The preference for transplanting kidneys with zero mismatches for HLA-A, -B, and -DR has been retained, while the importance of other numbers of mismatches has gotten less attention. New items in the algorithm are: waiting time on the transplantation list, 
matchability, distance between donor and recipient, and national import/export balance. Within one year after its introduction, its primary goals (increasing the number of transplantations for patients with long waiting times or poorly matchable HLA phenotype) were met ${ }^{37}$.

In Chapter 8, we analyzed the influence of HLA mismatch on renal graft outcome in our patient population using a strictly hierarchical model-fitting procedure for HLA-A, -B, and -DR mismatches. This procedure allows us to discriminate specific mismatch groups at risk for (or protected from) rejection or graft failure by analyzing the interaction between mismatches for different HLA loci. Patient survival and graft survival were analyzed for different time periods after transplantation. The conclusions that can be drawn from this study are that some specific HLA mismatches are related to rejection, whereas mismatch for HLA-B is related to long-term graft loss. Thus, HLA matching results in an important improvement in clinical outcome parameters after renal transplantation in patients using cyclosporine.

\section{Immunosuppression}

In the 1980s, cyclosporine-based immunosuppression became the standard immunosuppressive therapy for renal transplantation.

Usually cyclosporine was combined with steroids and azathioprine. With the exception of high-risk patients (retransplants, high \%PRA), patients were generally treated with the same immunosuppression during the cyclosporine era. Ideally, a more tailor-made immunosuppressive regimen should be applied.

Important side effects of cyclosporine are nephrotoxicity, hypertension, hypercholesterolemia, hirsutism, and gingival hyperplasia. For nearly 15 years, cyclosporine had no competitors. Because of the side effects and a 40\%-60\% acute rejection rate, newer immunosuppressives were sought. In recent years, tacrolimus and mycophenolate mofetil (MMF) have been introduced.

After the introduction of tacrolimus in Europe, our center joined a group of investigators who were designing and participating in multicenter trials with this drug. Tacrolimus-based immunosuppression was shown to be associated with a reduction in both acute and steroid-resistant rejection of $\pm 50 \%{ }^{38.39}$ compared to cyclosporine (Sandimmune)-based immunosuppression. However, the bioavailability and pharmacokinetics of Sandimmune are highly variable after oral administration. A new formulation of cyclosporine, Neoral, has since been introduced. This has been found to have significantly less pharmacokinetic variability. Data seem to indicate that cyclosporine/Neoral-based immuno-suppression has a lower incidence of rejection than cyclosporine/Sandimmune-based immunosuppression ${ }^{* 0}$. We were able to show that tacrolimus is also superior to cyclosporine/Neoral in preventing acute rejection and steroid-resistant rejection ${ }^{4 !}$.

Tacrolimus has also been successfully used as a rescue agent in salvaging allografts 
with refractory rejection under cyclosporine-based immunosuppression. Generally, $\pm 70 \%-80 \%$ of all grafts can be saved by conversion from cyclosporine to tacrolimus $^{2}$. This is a unique property of tacrolimus because there is hardly any counterpart in the opposite direction.

The safety profiles of tacrolimus and cyclosporine-based regimens are quite comparable (Chapter 7a). There is no difference in patient survival or in the incidence of infections or malignancies.

As for the side effects of the drugs, the nephrotoxic properties of both drugs are similar. Tremor and diarrhea are more frequently observed with tacrolimus, while hirsutism and gingival hyperplasia are almost exclusively observed during cyclosporine use. The incidence of hyperglycemia is significantly higher in tacrolimus-treated patients and is due to a dose-dependent and reversible decrease in C-peptide secretion ${ }^{43}$. Risk factors for developing diabetes mellitus are: high levels of tacrolimus, concomitant use of steroids, prediabetic state, race, and high body mass index. Dose reduction of tacrolimus usually leads to reversal of diabetes mellitus. The cardiovascular profile is much more favorable during the use of tacrolimus. There is better control of hypertension and lower levels of hypercholerolemia, even though there is significantly less use of statins after 3 years. The better cardiovascular profile may have beneficial long-term effects because both have been linked to cardiovascular disease and chronic transplant nephropathy.

Tacrolimus has steroid-sparing properties. A large proportion of patients can be weaned off corticosteroids. We have confirmed and extended these findings (Chapter 7c). Almost $90 \%$ of our patients could be weaned off steroids and more than $75 \%$ of these patients were on tacrolimus monotherapy.

Recently, mycophenolate mofetil (MMF) has been introduced. In combination with cyclosporine, it has been shown to be a potent immunosuppressive agent. Very little was known about the pharmacokinetics in recipients receiving tacrolimus. We have shown that MMF does not influence the absorption kinetics of tacrolimus (Chapter 7b). This suggests that no adjustment in tacrolimus dosing is necessary when MMF is administered concomitantly. Moreover, we were able to demonstrate that after 14 days, 1 gram of MMF may provide adequate systemic exposure when given in combination with tacrolimus.

The combination of tacrolimus and MMF was shown to be associated with a very low incidence of acute and steroid-resistant rejection (Chapter 7c).

Based on our experience, we have formulated dosing ${ }^{44.46}$ and management guidelines (Chapter 7c) for the use of tacrolimus in renal transplant recipients. 
The introduction of these newer drugs has resulted in a decrease in the number of rejections. Steroid-resistant rejection and vascular rejection are specially important risk factors for graft loss. Therefore, one can expect these newer drugs to improve long-term graft survival. Up until now, a significantly improved graft survival rate for patients treated with tacrolimus instead of cyclosporine has not been shown ${ }^{47}$, although a clear trend towards improvement has been reported ${ }^{48,49}$.

\section{Prevention of acute rejection and improvement in graft survival}

Rejections that result in structural damage to the graft, i.e., no return to baseline creatinine level or a high creatinine area under the curve (AUC) during the rejection period, are reported to have a particularly bad prognosis. These rejections usually present themselves as steroid-resistant or vascular rejections. Therefore, prevention of rejection is important in order to improve graft survival. This can be accomplished by pretransplant transfusion, prevention of sensitization (Chapter 3), and transplantation of well-matched kidneys (Chapter 8). Also, the use of newer immunosuppressives, such as the combination of tacrolimus with MMF (Chapter 7c), has been shown to contribute to a low rejection rate. One could argue that, with these powerful drugs, \%PRA and HLA matching not longer influence graft outcome. However, in a clinical trial with tacrolimus both factors were still found to have a significant influence on rejection rate ${ }^{50}$. Therefore, HLA matching is still important in the tacrolimus era. The incidence of rejection with an HLA-DRmatched kidney under tacrolimus-based immunosuppression is less than $20 \%$.

In Chapter 7c, we proposed a scheme for tailor-made immunosuppression in which low-risk recipients (first graft, HLA-DR-identical, and PRA $<20 \%$ ) can be treated with tacrolimus and low-dose corticosteroids. In the case of corticosteroidresistant rejection, MMF is added and patients are maintained on a triple-drug regimen. For the remaining patients, corticosteroids can be weaned in those who have not been treated for rejection. All other patients are initially treated with tacrolimus, low-dose corticosteroids and MMF. Hyperimmunized patients (PRA $>84 \%$ ) and patients with corticosteroid-resistant rejection are maintained on a triple-drug regimen. Recipients not requiring rejection therapy can be weaned off steroids after 3 months and MMF can be withdrawn after 6- 12 months.

\section{Long-term graft survival}

The parameter most predictive of long-term graft survival is the function of the graft at 1 year post-transplantation ${ }^{5-53}$. Well-functioning grafts have a lower rate of graft loss than less well-functioning grafts. In our multivariate analysis of factors influencing clinical outcome (Chapter 8), we confirmed and extended this finding. By linear regression analysis, we were able to discern factors related to the 1-year creatinine clearance. The most important factors negatively related to the prediction 
of 1-year graft function were: cold ischemia time, preservation with Eurocollins solution, donor age, and mismatch for HLA-B.

Mismatch for HLA-B is a risk factor for long-term graft survival in univariate, but not in multivariate, analysis when 1-year creatinine clearance is introduced into the model. Mismatch for HLA-B has been related to chronic rejection. Thus, it may be that this effect is already reflected in graft function at 1-year.

In contrast to general opinion, occurrence of acute rejection was not related to a decreased 1-year graft survival or to 1-year graft function in our study. This was probably due to our use of polyclonal antilymphocyte antibodies as first line treatment for acute rejection. The proportion of recurrent rejections was low $(12.2 \%)$, suggesting that we probably reversed acute vascular rejection and prevented the recurrence of acute rejections. Our explanation is supported by a recent report by Pelletier et al. ${ }^{\text {s4 }}$.

Not only 1-year creatinine clearance, but also the degree of proteinuria was associated with graft survival. Proteinuria is a consequence of graft damage, either pre-existent or occurring during the donation/transplantation procedure. Furthermore, it is related to steroid-resistant rejection and hypertension. Therefore, during the first year after transplantation the interaction between immunological and non-immunological factors determines long-term graft survival by balancing the result of input (quality of the graft) and early damaging events (preservation, steroid-resistant rejection, delayed graft function, hypertension). Consequently, it is essential to seek ways of improving the quality of the cadaveric graft. The prevention of damaging events is important. Optimal donor management and procurement procedure, improved preservation, matching for at least HLA-B and HLA-DR, and shortening of the cold ischemia time are the areas to be targeted. Cold ischemia time is the one that can easily be shortened. The influence of cold ischemia time has been underestimated. It was thought to be related to an increase in delayed graft function only and not to long-term sequelae. Moreover, there are indications that the beneficial effect of matching is completely offset by the negative influence of a long cold ischemia timess.

The duration of cold ischemia depends on allocation (including typing and crossmatching), transport, and in-hospital procedures. In the early days of matching, HLA-typing was performed on peripheral blood. Results were often available before the kidneys were removed. Later, spleen lymphocytes were used. They provide a better quality of HLA class II typing. Therefore, typing results are available at the earliest 8 hours after the kidney has been removed. The introduction of DNA techniques has made it possible to perform reliable HLA class I and II typing on peripheral blood and can be performed before removal of the donor kidney. Thus, a widespread application of this approach may shorten cold ischemia time considerably. Recently, the need for crossmatching in patients who 
have always been negative in the screening procedures has been questioned ${ }^{56,57}$. In these cases, if no crossmatch is performed, cold ischemia time may also be shortened. Transportation time can be shortened when more kidneys are transplanted locally. With the exception of mandatory exchange for zero HLA-A, B, and -DR mismatch kidneys and highly immunized patients in special programs like Acceptable Mismatch and HIT, it is possible to transplant a reasonable proportion of the kidneys locally with at least one HLA-B and one HLA-DR match. If the minimal match cannot be provided, kidneys should be offered for exchange.

An example of how altered allocation can reduce cold ischemia time is the Eurotransplant Senior Program. The purpose of this program is to shorten the cold ischemia time for kidneys that are vulnerable to ischemia due to the advanced age of the donor. Cold ischemia time is shortened by local use of kidneys of older donors ( $>65$ years) for older recipients. Since its introduction, the mean cold ischemia time of these grafts has been reduced from \pm 19 hours to \pm 12 hours (personal communication G. Persijn, Eurotransplant). Thus, the goal of limiting cold ischemia time for these vulnerable grafts has been met. In fact, it seems that shortening the time between donation and grafting is important for both immediate and long-term function for all kinds of kidneys. It would, therefore, be advisable to give this more attention during the allocation process.

Last, but not least, is improvement of in-hospital procedures. Renal transplantation should be regarded as an urgent operation rather a semi-urgent one that can be delayed for half a day or longer. Taken together, these measures may improve graft survival. 


\section{References}

1. Waiser J, Budde K, Schreiber M, Peibst O, Koch U, Bohler T, Hoffken B, Hauser I, Neumayer $\mathrm{HH}$. The quality of life in end stage renal disease care. Transpl Int 1998; 11 Suppl 1: S42-S5.

2. Schnuelle P, Lorenz D, Trede M, van der Woude FJ. Impact of renal cadaveric transplantation on survival in end-stage renal failure: evidence for reduced mortality risk compared with hemodialysis during long-term follow-up. J Am Soc Nephrol 1998; 9: 2135-41.

3. Opelz G, Sengar DPS, Mickey MR, Terasaki PI. Effect of blood transfusions on subsequent kidney transplants. Transplant Proc 1973; 5: 253-9.

4. van Hooff JP, Kalff MW, Poelgeest AE, Persijn GG, van Rood JJ. Blood transfusions and kidney transplantation. Transplantation 1976; 22: 306-7.

5. Persijn GG, Cohen B, Lansbergen Q, van Rood J. Retrospective and prospective studies on the effect of blood transfusions in renal transplantation in the Netherlands. Transplantation 1979; 28: 396-401.

6. Opelz G, Vanrenterghem Y, Kirste G, Gray DWR, Horsburgh T, Lachance JG, Largiader F, Lange H, Vujaklija-Stipanovic K, Alvarez-Grande J, Schott W, Hoyer J, Schnuelle P, Descoeudres C, Ruder H, Wujciak T, and Schwarz V. Prospective evaluation of pretransplant blood transfusions in cadaver kidney recipients. Transplantation 1997; 63: 964-7.

7. Lagaaij EL, Hennemann IPH, Ruigrok M, de Haan MW, Persijn GG, Termijtelen A, Hendricks GF, Wiemar W, Claas FH, van Rood JJ. Effect of one-HLA-DRantigen-matched and completely HLA-DR-mismatched blood transfusions on survival of heart and kidney allografts. N Eng J Med 1989; 321(11): 701-705.

8. Bayle F, Masson D, Zaoui P, Vialtel P, Janbon B, Bensa JC, Cordonnier DJ. Beneficial effects of one HLA haplo- or semi-identical transfusion versus three untyped blood units on alloimmunization and acute rejection episodes in first renal allograft recipients. Transplantation 1995; 59: 719-23.

9. Middleton D, Martin J, Douglas J, McClelland M. Transfusion of one HLA-DR antigen-matched blood to potential recipients of a renal allograft. Transplantation $1994 ; 58(7)$ : 845-8.

10. van der Mast BJ, Balk AHMM. The effect of HLA-DR-shared blood transfusion on the clinical outcome of heart transplantation. Transplantation 1997; 63: 1514-9.

11. van Twuyver E, Mooijaart RJ, ten Berge IJ, van der Horst AR, Wilmink JM, Kast WM, Melief CJM, de Waal LP. Pretransplant blood transfusion revisited. N Engl J Med 1991; 325: 1210-3.

12. Young NT, Roelen DL, Iggo N, Gray WG, Roake JA, Graham V, Wood KJ, Dallman MJ, Welsh KI, Morris PJ. Effect of one-HLA-haplotype-matched and HLA-mismatched blood transfusions on recipient $T$ lymphocyte allorepertoires. Transplantation 1997; 63: 1160-5.

13. Baudouin V, de Vitry N. Hiesse C, Lang P, Bloch J, Legouvello S, Sterkers G. Cytotoxic $T$ lymphocyte changes after HLA-DR match and mismatch blood transfusion. Transplantation 1997: 63: 1155-60. 
14. Opelz G. Collaborative transplant study kidney exchange trial for highly sensitized patients. Clin Transpl 1991: 61-4.

15. Cook DJ, Terasaki PI, Iwaki Y, Terashita GY, Lau M. An approach to reducing early kidney transplant failure by flow cytometry crossmatching. Clin Transplantation 1987; 1: 253-6.

16. Cook DJ, Terasaki PI, Iwaki Y, Terashita G, Fujikawa J, Gera J, Takeda A, Danovitch G, Rosenthal JT, Fine R, Ettenger R, Mendez R, Dainko E, Kavalich A, Martin D, Teichman S, Ward H, Koyle M, Berne T, Brennan LP, Lieberman E, Cohen A, Strauss F. Flow cytometry crossmatching for kidney transplantation. In: Terasaki PI, ed. Clinical Transplants 1988, Los Angeles, UCLA Tissue Typing Laboratory. 1989: 375-80.

17. Kerman RH, Van Buren CT, Lewis RM, DeVera V, Baghdahsarian V, Gerolami K, Kahan BD. Improved graft survival for flow cytometry and antihuman-globuline cross-match negative retransplantation recipients. Transplantation 1990; 49: 52-6.

18. Talbot D, Givan AL, Shenton BK, Stratton A, Proud G, Taylor RMR. The relevance of a more sensitive cross match-assay to renal transplantation. Transplantation 1989; 47: 552-5.

19. Thistlethwaite JR, Buckingham M, Stuart JK, Gabor AO, Mayes JT, Stuart FP. T-cell immunofluorescent flow cytometry crossmatch results in cadaver donor renal transplantation. Transplant Proc 1987; 19: 722-4.

20. Ogura K, Terasaki PI, Johnson C, Mendez R, Rosenthal JT, Ettenger R, Martin DC, Dainko E, Cohen L, Mackett T, Berne T, Barba L, Lieberman E. The significance of a positive flow cytometry crossmatch test in primary kidney transplantation. Transplantation 1993; 56: 294-8.

21. Martin S, Dyer PA, Mallick NP, Gokal R, Johnson RW, Posttransplant anti-donor lymphocytotoxic antibody production in relation to graft outcome. Transplantation 1987; 44: 50-3.

22. Christiaans MHL, Nieman F, van Hooff JP, van den Berg-Loonen EM. HLA-Class I and II antibodies by ELISA and CDC before and after transplantation. Transplantation (in press).

23. Lobo PI, Spencer CE, Stevenson WC, Pruett TL. Evidence demonstrating poor kidney graft survival when acute rejections are associated with IgG donor-specific lymphocytotoxin. Transplantation 1995; 59: 357-60.

24. Scornik J, Salomon D. Lim P. Howard R, Pfaff W. Posttransplant donor antibodies and graft rejection. Evaluation by two-color flow cytometry. Transplantation 1989; 47: 287-90.

25. Opelz G. Importance of HLA antigen splits for kidney transplant matching. Lancet 1988: $2 ; 61-4$.

26. Opelz G, Mytilineos J, Scherer S, Dunckley H, Trejaut J, Chapman J, Middleton D, Savage D, Fischer O, Bignon JD, Bensa JC, Albert E, Noreen H. Survival of DNA HLA-DR typed and matched cadaver kidney transplants. The collaborative transplant study. Lancet 1991: 338: 461-3. 
27. Sanfilippo F, Vaughn WK, Spees EK. Heise ER, Lefor WM. The effect of HLA-A,B matching on cadaver renal allograft rejection using public versus private specificities. Transplantation $1984 ; 38: 483-9$.

28. van Hooff JP, van Leeuwen A, Paul L, Leunissen KML, Lecomte C, D'Amaro J, Cohen B, Alexandre GRJ, van Rood J. The influence of matching for broadly reacting antigens on long-term kidney graft survival. Transplant Proc 1985; 17: 2205-6.

29. Christiaans MHL, van den Berg-Loonen PM, Nieman FHM, van Hooff JP. Very low frequency of rejection due to $\mathrm{HLA}-(\mathrm{A}+) \mathrm{B}+\mathrm{DQ}$ matching. Transplant Proc 1992; 24: 2441-2.

30. Christiaans MHL, Peltenburg HG, Nieman FHM, Kootstra G, Lavrijsen ATJ, van den Berg-Loonen PM, Tiebosch A, Leunissen KML, van Hooff JP. The impact of ischemic lesions in the donor kidney, donor age, recipient age and HLA (A, B, C, DR, DQ) matching on clinical course after kidney grafting. Transpl Int 1992; 5 : S627-S8.

31. Doxiadis II, Smits JM, Schreuder GM, Persijn GG, van Houwelingen HC, van Rood J. Claas FH. Association between specific HLA combinations and probability of kidney allograft loss: the taboo concept. Lancet 1996; 348: 850-3.

32. Claas FH, van Leeuwen A, van Rood JJ. Hyperimmunized patients do not need to wait for an HLA identical donor. Tissue Antigens 1989; 34: 23-9.

33. Claas FH. Negative cross-match selection of HLA-DR compatible kidneys for highly sensitised patients. Immunol Lett. 1991; 29: 61-4.

34. Thorogood J, Persijn GG, Schreuder GMTh, D'Amaro J, Zantvoort FA, van Houwelingen JC, van Rood JJ. The effect of HLA matching on kidney graft survival in separate posttransplantation intervals. Transplantation 1990; 50: 146-50.

35. Gilks WR, Gore SM, Bradley BA. Renal transplant rejection: transient immunodominance of HLA mismatches. Transplantation 1990; 50: 141-6.

36. Wujciak T, Opelz G. A proposal for improved cadaver kidney allocation. Transplantation 1993; 56: 1413-517.

37. De Meester J, Persijn GG, Wujciak T, Opelz G, Vanrenthergem Y. The new Eurotransplant kidney allocation system. Transplantation 1998; 66: 1154-9.

38. Schleibner S, Krauss M, Wagner K, Erhard J, Christiaans M, van Hooff J, Buist L. Mayer D. FK506 versus cyclosporin A in the prevention of renal allograft rejection European pilot study: six-week results. Transpl Int 1995; 8: 86-90.

39. Mayer AD, Dmitrewski J, Squifflet JP, Bessen T, Grabensee B, Klein B, Eigler FW, Heemann U, Pichlmayr R, Behrend M, Vanrenterghem Y, Donck J, van Hooff J, Christiaans M, Morales JM, Andres A, Johnson RWG. Short C, Buchholz B, Rehmert N, Land W, Schleibner S, Forsythe JLR, Talbot D, Neumayer HH, Hauser I, Ericzon BG, Brattstrōm C, Claesson K, Mühlbacher F, Pohanka E. Multicenter randomized trial comparing tacrolimus (FK506) and cyclosporine in the prevention of renal allograft rejection. Transplantation 1997; 64: 436-43.

40. Shah MB, Martin JE, Schroeder TJ, First MR. The evaluation of the safety and tolerability of two formulations of cyclosporine: Neoral and Sandimmune. Transplantation 1999: 67: 1411-7. 
41. Christiaans M, van Hooff J, van Duijnhoven E. Single center experience of tacrolimus versus cyclosporine-neoral in renal transplantation. Kidney Int 1999; 55: 2598.

42. Jordan ML, Shapiro R, Vivas C, Scantlebury V, McCauley J, McMichael J, Randhawa P, Demetris A, Fung J, Starzl TE. Outcome of tacrolimus conversion therapy for renal allograft rejection: 5-year follow-up. Transplant Proc in press.

43. van Hooff JP, van Duijnhoven EM, Christiaans MHL. Tacrolimus and glucose metabolism. Transplant Proc in press.

44. van Duijnhoven E, Christiaans M, Schafer A, Undre N, van Hooff J. Tacrolimus dosing requirements in diabetic and nondiabetic patients calculated from pretransplantation data. Transplant Proc 1998; 30: 1266-7.

45. van Duijnhoven E, Christiaans M, Undre N, Stevenson P, van Hooff J. The effect of breakfast on the oral bioavailability of tacrolimus in diabetic and nondiabetic patients before transplantation. Transplant Proc 1998; 30: 1268-70.

46. Christiaans M, van Duijnhoven E, Beysens T. Undre N, Schafer A, van Hooff J. Effect of breakfast on the oral bioavailability of tacrolimus and changes in pharmacokinetics at different times posttransplant in renal transplant recipients. Transplant Proc 1998; 30: 1271-3.

47. Opelz G. Effect of immunosuppressive therapy on graft half-life projections. Transplant Proc in press.

48. Mayer $\mathrm{AD}$ for the European tacrolimus multicenter renal study group. Four-year follow-up of the European tacrolimus multicenter renal study. Transplant Proc in press.

49. Jensik SC for the FK506 Kidney transplant study group. Tacrolimus (FK506) in kidney transplantation: three-year survival results of the US multicenter, randomized, comparative trial. Transplant Proc 1998; 30: 1216-8.

50. Richards E, Schleibner S, Talbot D, and the European tacrolimus multicenter renal study group. An exploratory analysis of prognostic factors for patient outcome during the first year following renal transplantation. Transplant Proc 1998; 30: 1386-8.

51. Vanrenthergem Y, Peeters J: In Touraine JL, et al (eds): Late graft loss. Great Brittain: Kluwer Academic Publishers: 1997: 85.

52. Kasiske BL. Clinical correlates to chronic renal allograft rejection. Kidney Int 1997. 52 (suppl 63): S71-S4.

53. Kuo PC, Johnson LB, Schweitzer EJ, Alfrey EJ, Waskerwitz J, Bartlett ST. Utilization of the older donor for renal transplantation. Am J Surg 1996, 172: $551-7$.

54. Pelletier RP, Cosio F, Henry ML, Bumgardner GL, Davies EA, Elkhammas EA, Ferguson RM. Acute rejection following renal transplantation. Evidence that severity is the best predictor of subsequent graft survival time. Clin Transplantation 1998: 12: 543-52. 
55. Fuggle $\mathrm{S}$ on behalf of Members of the Kidney Advisory Group, UKTSSA. The effect of HLA-A, B, DR mismatches on kidney transplant survival in the UK. Poster XVII World Congress of the Transplantation Society, Montreal, July 12-17 1998.

56. Kerman RH, Susskind B, Ruth J, Katz S, van Buren CT, Kahan BD. Can an immunologically, nonreactive potential allograft recipient undergo transplantation without a donor-specific crossmatch? Transplantation 1998; 66: 1833-4.

57. Matas AJ, Sutherland DER. Kidney transplantation without a final crossmatch. Transplantation 1998; 66: 1835-6. 


\section{Summary and concluding remarks}

Renal transplantation has evolved in the last decades from an experimental treatment of end-stage renal disease for few selected patients to the preferred treatment for the majority of patients with end-stage renal disease.

A clinical renal transplantation program involves teamwork in which transplant immunologist, surgeon, and nephrologist participate. Generally, the surgeon is responsible for the donor program and the surgical procedure. The nephrologist is more involved in patient selection, pre- and postoperative care, and the handling of immunosuppression. The transplant immunologist is in charge of the transfusion protocol, antibody screening, HLA matching, and crossmatching. Various relevant aspects of these disciplines are dis-cussed in chapter 1. Important end-points for most strategies are acute rejection rate and graft survival. Immunological procedures to decrease the rate of rejection and increase graft survival are pretransplant transfusion, screening for anti-HLA antibodies in serum, matching for HLA-A, -B, and -DR, and crossmatching. Clinical procedures to reduce the rate of rejection and to increase graft survival include optimal preservation of the graft, administration of immunosuppressive drugs, and preventing (a)specific injury to the graft.

Screening for HLA antibodies and matching for HLA antigens have played a central role in renal allografting since the 1960s. However, management of recipients, including immunosuppressives, have changed considerably and new histocompatibility techniques are introduced. Therefore, both standard and new techniques have to be compared with each other and with clinical outcome parameters to evaluate their value. We have studied several of these aspects of which the results are described in the different chapters.

It has been shown that patients who received blood transfusion before transplantation have a decreased acute rejection rate and improved renal graft survival compared to patients who never received a blood transfusion. It was hypothesized that this beneficial effect is due to matching between transfusion donor and recipient for HLA-DR. Moreover, by DR-matching anti-HLA antibodies were less frequently detected after transfusion. These studies had methodological shortcomings (small sample size; use of different number of transfusions in DRmatched and nonmatched patients; control group consisting of patients receiving random transfusion(s) instead of nonmatched transfusion; detection of panelreactive, instead of donor-directed antibodies). Therefore, we studied in chapter 2 the incidence of donor-directed anti-HLA antibodies after transfusion of a single unit of leukocyte-poor erythrocyte-concentrate in immunologically naive patients. 
We have tried to answer the following questions: What is the incidence of donordirected HLA antibodies after such a transfusion? Is there a difference in occurrence of these antibodies between patients receiving DR-matched blood transfusions and patients receiving nonmatched blood transfusion? Is there a difference in acute rejection rate or graft survival after transplantation between patients receiving DR-matched and nonmatched blood transfusion? Out of the 247 patients, 107 received a DR-matched transfusion, while 140 received a nonmatched transfusion. Alloreactive T-cell antibodies were detected in $6.5 \%$ of the patients, while alloreactive B-cell antibodies were detected in $17.3 \%$ of the patients. There was no significant difference in the incidence of these antibodies between DRmatched and DR-nonmatched patients. In multivariate analysis, DR-matching between transfusion donor and recipient was no risk factor for either rejection or graft survival. From this study, we conclude that transfusion of a single unit of leukocyte-poor erythrocytes results in a low incidence of T-cell antibodies, while the incidence of B-cell antibodies is higher. The development of both types of antibodies is irrespective of DR-match. Rejection rate and graft survival is independent of DR-match between transfusion donor and recipient.

Screening sera for presence of anti-HLA antibodies is usually performed by the complement-dependent-cytotoxicity assay; for T-cell antibodies the NIH assay, for B-cell antibodies the TCF assay. A new assay detecting specific anti-HLA IgG is Prastat, an enzyme-linked immunosorbant assay (ELISA). Both assays record as well the percentage panel reactive antibodies (PRA) as HLA specificity. We tried to answer the following questions: What is the method agreement between ELISA and NIH/TCF CDC screening? Do both screening techniques differ regarding result of the screening and acute rejection rate or graft survival? In chapter $\mathbf{3}$, we have compared the results of ELISA and CDC (class I and II) in 429 sera drawn pre- and post-transplantation in 143 recipients of a renal allograft and immunosuppressed by cyclosporine. The method agreement between ELISA and $\mathrm{NIH}$, and between ELISA and TCF, was too low to replace one assay by the other. Furthermore, we have correlated the results of the assays with graft survival and rejection-free survival. None of the assays was related to graft survival. Rejectionfree survival was related to the results of NIH and ELISA in both pre- and posttransplant sera. Change in PRA from pretransplant to posttransplant serum determined by NIH correlated better with rejection-free survival than by ELISA. Compared to ELISA-negative sera, sera with class I specificity were related to rejection, even when these antibodies were not donor-directed. ELISA-positive sera without attributed specificity were not at risk for rejection. For NIH, \%PRA and not specificity, was a risk factor for rejection. Class II antibodies were not related to rejection in any assay. From this study, we conclude that ELISA and NIH are complementary screening techniques. They are of equal predictive value for 
rejection. The optimal strategy on combining these techniques has to be determined.

Before transplantation, a crossmatch is performed between lymphocytes of the donor and serum of the donor. A negative T-cell complement-dependentcytotoxicity crossmatch (NIH crossmatch) is mandatory. The flow cytometry (FC) crossmatch is a technique that is more sensitive and IgG specific. In chapter $\mathbf{4}$, the principles of FC and the validation of the FC crossmatch as used in the University Hospital Maastricht is presented, together with an overview of the literature on the comparison between CDC crossmatch and FC crossmatch. Positivity for FC crossmatch was defined as test result (ratio of test serum and negative control serum) higher than the mean +3 SD of the result of negative control sera. The FC crossmatch was shown to be T-cell specific and at least 9 dilution steps more sensitive than the NIH crossmatch.

In chapter 5, we tried to find the answer to the question: What is the relationship between pretransplant FC crossmatch and acute rejection and 1-year renal graft survival in patients transplanted with a negative HLA class I CDC crossmatch (NIH)? The study population consisted of patients thoroughly screened by CDC and transplanted with a negative NIH crossmatch with peak and current serum. In 67 non-sensitized patients, 4 had a positive FC crossmatch without acute rejection or 1-year graft loss. In 76 patients at risk (retransplants and/or sensitized) 21 showed a positive FC crossmatch. However, the 1-year graft survival and acute rejection rate were comparable between the FC-positive and FC-negative patients ( $76 \%$ vs. $80 \%$, and $48 \%$ vs. $46 \%$, respectively). The conclusion of this study was that in immunologically well-documented patients FC crossmatch was not advantageous. In $28 \%$ of our patients at risk, the FC crossmatch result was positive with current and/or peak serum. These patients would have been denied to be transplanted with their graft, while they are in fact doing very well.

Donor-specific antibodies can develop after transplantation. Their role in renal transplant outcome has been questioned. In chapter 6, we tried to answer the question: Do FC and CDC crossmatch with post-transplant serum differ with respect to acute rejection rate or renal graft survival? Current and post-transplant sera were tested from 143 renal allograft recipients transplanted with a negative current NIH crossmatch. By NIH, $6.1 \%$ of the patients were antibody-positive. They had a significantly lower 1-year graft survival compared to antibody-negative patients $(37.5 \%$ vs. $86.2 \%)$ and were treated more frequently for rejection $(100 \%$ vs. $37.4 \%)$.

In contrast, by FC $9.8 \%$ of the patients were antibody-negative before and antibody-positive after transplantation, $79 \%$ were antibody-negative both pre- and 
post-transplantation, and $11.2 \%$ were antibody-positive already before transplantation. Patients who were antibody-negative before and antibody-positive after transplantation, had a lower graft survival and rejection-free survival than both other groups in Cox regression analysis. By relating the FC result of posttransplant serum with that of pretransplant serum, the relative change in fluorescence ratio (RCFR) was determined. It was shown that the $25 \%$ of patients with the highest increase in RCFR were at a comparable increased risk for both graft failure and acute rejection as the $9.8 \%$ of patients that were antibody-positive according to the standard definition. Thus, by defining antibody-positivity by RCFR, a large group of patients can be discerned that is at increased risk for unfavourable graft outcome. The conclusion of this study was that, despite its increased sensitivity, the standard definition of antibody-positivity by FC does not result in discerning a much larger group of patients at risk for rejection and/or graft failure than antibody-positivity by CDC. However, by our new definition of positivity, based on relative change in fluorescence ratio, a much larger group of patients is shown to be at risk. When this result is confirmed in a prospective study, patients at risk for graft failure may be recognized early after transplantation and specific therapy may be targeted.

In recent years, newer immunosuppressives have been introduced. In chapter 7a, an overview of literature is given on the use of tacrolimus in renal transplantation. Treatment with tacrolimus, compared to cyclosporine, results in a decrease in both acute and corticosteroid-resistant rejection rate of $\pm 50 \%$ with the possibility of better graft survival. Although the safety profiles are comparable, there are differences in side effects that are important. Patients on cyclosporine are reported to have a higher incidence of gum hyperplasia and hirsutism. Patients on tacrolimus are reported to have a better cardiovascular risk profile (less hypertension and less hypercholesterolemia). However, hyperglycemia is more frequently reported in patients on tacrolimus.

Tacrolimus is generally combined with steroids. Especially this combination with mycophenolate mofetil (MMF) is very potent. Very little is known about the pharmacokinetics of MMF in organ transplant recipients receiving tacrolimus-based immunosuppressive regimen. In chapter $\mathbf{7 b}$, we have studied in a multicenter trial the pharmacokinetics of mycophenolate mofetil (MMF) in combination with tacrolimus. It was shown that the pharmacokinetics of tacrolimus was unchanged in presence of MMF. Moreover, the dose of MMF may be decreased with time posttransplant to maintain stable systemic exposure to its pharmacologically active form mycophenolic acid.

In chapter 7c, dosing and management guidelines for tacrolimus in renal transplant 
patients are given. Generally, with the exception of hyperimmunized patients, patients are receiving the same basic immunosuppression. Based on our experience, immuno-suppression can be adjusted to specific clinical situation for every patient. Therefore, a proposal for tailor-made immunosuppression is presented.

The outcome of renal allografting is determined by many factors. Therefore, we have investigated in chapter 8 which immunologic and non-immunologic factors influence outcome parameters in the cohort of 346 cadaveric renal transplantations on a cyclosporine-based immunosuppressive regimen transplanted at the University Hospital Maastricht. By multivariate analysis, immunological and nonimmunological factors were analyzed for their relation to clinical outcome. Besides acute rejection rate and graft survival, also patient survival, the occurrence and duration of delayed graft function (DGF), duration of hospitalization in the first year after transplantation, and creatinine clearance at year 1 were outcome parameters. The influence of histocompatibility was modest. Matching for some HLA loci resulted in better outcome only for the parameters rejection and creatinine clearance at year 1 . Panel reactive antibodies and previous transplants were other immunological factors that influenced DGF and short-term patient and graft surival. Other factors than histocompatibility (donor, preservation, and recipient related) were related to short- and long-term outcome parameters. Some of these may be influenced by a change in donor and recipient management. Especially, prevention of DGF by reducing CIT and modifying pretransplant dialysis may improve renal transplant outcome.

In chapter 9, the results of this study are discussed in relation to existing immunological and clinical strategies in renal transplantation. Several proposals for adjustments of these strategies are made. 


\section{Samenvatting}

Niertransplantatie heeft zich de laatste decennia ontwikkeld van een experimentele therapie voor een beperkt aantal geselecteerde patiënten tot de voorkeursbehandeling voor de overgrote meerderheid van patiënten met eindstadium nierfalen.

Een klinisch niertransplantatie programma is het resultaat van samenwerking van transplantatie immunoloog, chirurg en nefroloog. Over het algemeen is de chirurg de eindverantwoordelijke voor het donatie programma en de operatie. De nefroloog is degene die zorgdraagt voor de patiëntenselectie, de zorg voor de patiënten voor en na de operatie, en de medicatie, inclusief immuunsuppressiva (medicijnen ter voorkoming van afstoting van de nier). In hoofdstuk 1 worden verscheidene relevante aspecten van deze disciplines besproken. De belangrijkste eindpunten voor de meeste strategieên zijn de frequentie van acute afstoting (rejectie) en transplantaat overleving. Immunologische maatregelen om rejecties te voorkomen en transplantaat overleving te verhogen zijn bloedtransfusie vóór de transplantatie, screenen op de aanwezigheid van HLA antistoffen in het serum van de ontvanger, matchen voor de weefseleigenschappen HLA-A, -B en -DR, en het verrichten van kruisproeven. Klinische maatregelen om dit te bereiken bevatten o.a. optimale preservatie van het transplantaat, toediening van immuunsuppressieve geneesmiddelen, en het voorkomen van (a)specifieke schade aan het transplantaat.

Screenen op HLA antistoffen en matchen voor HLA antigenen spelen sinds de zestiger jaren een centrale rol in niertransplantatie. Echter, de behandeling van ontvangers, inclusief de toegediende immuunsuppressiva, is sterk veranderd en nieuwe technieken om overeenkomsten in weefseleigenschappen (histocompatibiliteit) te bepalen zijn geïntroduceerd. Nieuwe technieken dienen met standaard technieken vergeleken te worden en met klinische uitkomst parameters om hun nut te bepalen. Wij hebben verscheidene van deze aspecten bestudeerd, waarvan de resultaten in de diverse hoofdstukken zijn beschreven.

Het is aangetoond, dat patiënten die vóór transplantatie getransfundeerd zijn een verminderde rejectie frequentie en verbeterde transplantaat overleving hebben t.o.v. patiënten die nooit getransfundeerd zijn. Er bestaat een hypothese dat dit gunstige effect een gevolg is van het matchen tussen transfusiedonor en -ontvanger voor HLA-DR. Bovendien zijn er minder frequent HLA antistoffen gevonden na transfusie door te matchen voor DR. Deze studies hadden methodologische tekortkomingen (klein aantal patiënten; verschillend aantal transfusies in ontvangers van DR-gematcht en niet-gematcht bloed; detectie van antistoffen in een panel van cellen i.p.v. tegen donorcellen). Daarom hebben wij in hoofdstuk 2 de incidentie 
bestudeerd van donor-gerichte HLA antistoffen na transfusie van 1 eenheid leucocyten-arm erythrocyten-concentraat in immunologisch 'blanco' patiënten. We hebben geprobeerd de volgende vragen te beantwoorden: Wat is de incidentie van donor-gerichte HLA antistoffen na zo'n transfusie? Is er een verschil in het ontstaan van deze antistoffen tussen patiënten die een DR-gematchte en nietgematchte transfusie ontvingen? Is er een verschil in het optreden van acute rejectie of transplantaat overleving tussen patiënten die een DR-gematchte en niet-gematchte transfusie ontvingen? Van de 247 patiënten ontvingen er 107 een DR-gematchte transfusie en 140 een niet-gematchte transfusie. Alloreactieve T-cel antistoffen ontstonden in $6.5 \%$ van de patiënten, terwijl alloreactieve B-cel antistoffen in $17.3 \%$ van de patiènten aangetoond konden worden. Er was geen significant verschil in incidentie van deze antistoffen tussen patiënten met een DR-gematchte en niet-gematchte transfusie. In een multivariaat analyse bleek het matchen voor DR tussen transfusiedonor en -ontvanger geen risicofactor te zijn voor het optreden van rejectie dan wel transplantaat verlies. Wij concluderen uit deze studie, dat transfusie van I eenheid leucocyten-arm erythrocyten-concentraat resulteert in een lage incidentie van T-cel antistoffen, terwijl de incidentie van B-cel antistoffen hoger is. Het ontstaan van beide types antistoffen is onafhankelijk van het matchen voor DR. Ook het optreden van rejectie en de transplantaat overleving is onafhankelijk van matchen voor DR tussen transfusiedonor en -ontvanger.

Het screenen van sera op de aanwezigheid van HLA antistoffen gebeurt gewoonlijk m.b.v. de complement-afhankelijke cytotoxiciteits (CDC) test; voor T-cel antistoffen is dit de NIH test, voor B-cel antistoffen de TCF test. Een nieuwe test welke specifiek anti-HLA IgG aantoont, is Prastat, een 'enzyme-linked immunosorbant assay' (ELISA). Beide technieken tonen zowel het percentage antistoffen dat met een panel reageert (PRA) als de HLA specificiteit. We probeerden de volgende vragen te beantwoorden: Wat is de mate van overeenkomst in de test-uitslag (method agreement) tussen ELISA en NIH/TCF? Verschillen beide technieken in de relatie tussen test-uitslag en acute rejectie frequentie of transplantaat overleving? In hoofdstuk 3 hebben wij de resultaten van ELISA en CDC (klasse I en II) vergeleken in 429 sera, afgenomen voor en na niertransplantie. Alle 143 patiënten kregen cyclosporine als immunosuppressie. De method agreement tussen ELISA en NIH, en tussen ELISA en TCF, was te gering om elkaar te vervangen. Daarnaast zijn de resultaten van de testen gecorreleerd met transplantaat en rejectie-vrije overleving. Geen enkele test gaf een relatie tussen uitslag en transplantaat overleving. Rejectie-vrije overleving was gerelateerd met het testresultaat van NIH en ELISA met sera afgenomen vóor en na transplantatie. Voor wat betreft de rejectie-vrije overleving correleerde verandering in PRA tussen sera afgenomen vóór en na transplantatie beter indien deze bepaald was m.b.v. NIH dan met ELISA. Vergeleken met ELISA-negatieve sera, waren ELISA- 
positieve sera met klasse I specificiteit gerelateerd met rejectie, zelfs indien de antistoffen niet gericht waren tegen donorantigenen. Een positieve ELISA-uitslag zonder dat specificiteit toegewezen kon worden, was geen risico factor voor rejectie. Voor NIH geldt dat PRA een risico factor was voor rejectie en niet de specificiteit. In geen enkele test waren klasse II antistoffen gerelateerd aan rejectie. De conclusie van deze studie was, dat ELISA en NIH complementaire screening technieken zijn. Zij voorspellen in gelijke mate rejectie. De optimale strategie om ze te combineren dient echter nog onderzocht te worden.

Vóór transplantatie wordt een kruisproef verricht tussen lymfocyten van de donor en serum van de ontvanger om de aanwezigheid van donor-gerichte antistoffen uit te sluiten. Een negatieve T-cel CDC kruisproef (NIH kruisproef) is verplicht. De 'flow cytometry' (FC) kruisproef is een gevoeliger techniek en IgG specifiek. In hoofdstuk 4 zijn de principes van de FC en de validatie van de FC kruisproef, zoals gebruikt in het academisch ziekenhuis Maastricht, gepresenteerd tezamen met een overzicht van de literatuur betreffende de vergelijking van CDC en FC kruisproeven. Een positieve FC kruisproef is gedefinieerd als een uitslag (ratio van test serum en negatieve controle serum) groter dan het gemiddelde +3 SD van de uitslag van negatieve controle sera. Aangetoond werd, dat de FC kruisproef T-cel specifiek is en minstens 9 dilutie-stappen gevoeliger dan de NIH kruisproef.

In hoofdstuk 5 probeerden wij een antwoord te vinden op de vraag: Wat is de relatie tussen de uitslag van de FC kruisproef vór transplantatie en het optreden van acute rejectie en 1-jaars transplantaat overleving in patiënten die getransplanteerd zijn met een negatieve HLA klasse I CDC kruisproef $(\mathrm{NIH})$ ? De studie-populatie bestond uit patiënten die grondig gescreend waren m.b.v. CDC en getransplanteerd met een negatieve NIH kruisproef met serum met de hoogste PRA (peak) en serum t.t.v. transplantatie afgenomen (current). Vier van de 67 nietgeïmmuniseerde patiënten had een positieve FC kruisproef zonder acute rejectie of 1-jaars transplantaat verlies. Van de 76 patiënten met een verhoogd risico (meerdere transplantaten en/of geïmmuniseerd) hadden 21 een positieve FC kruisproef. De 1-jaars transplantaat overleving en het optreden van acute rejectie was echter vergelijkbaar tussen FC-positieve en FC-negatieve patiënten ( $76 \%$ vs. $80 \%$, respectievelijk $48 \%$ vs. $46 \%$ ). De conclusie van deze studie was dat in immunologisch goed-gedocumenteerde patiēnten de FC kruisproef geen toegevoegde waarde had. In $28 \%$ van onze patiënten met een verhoogd risico was de FC kruisproef positief met current en/of peak serum. Deze patiënten zouden hun huidige transplantatie ten onrechte onthouden zijn, indien de FC kruisproef bepalend was.

Donorspecifieke antistoffen kunnen ontstaan na transplantatie. Er bestaat discussie 
over hun rol in de transplantaat uitkomst. In hoofdstuk 6 probeerden we om de volgende vraag te beantwoorden: Verschillen de uitslagen van de FC en CDC kruisproef met serum afgenomen na transplantatie in hun relatie met het optreden van acute rejectie en transplantaat overleving? Getest zijn de sera afgenomen t.t.v. de transplantatie (current) en na transplantatie (post) van 143 niertransplantaat ontvangers getransplanteerd met een negatieve NIH kruisproef met current serum. Van de patiënten was $6.1 \%$ antistof-positief m.b.v. de $\mathrm{NIH}$. $\mathrm{Zij}$ hadden een significant lagere 1-jaars transplantaat overleving t.o.v. antistof-negatieve patiënten $(37.5 \%$ vs. $86.2 \%)$ en werden vaker behandeld voor een acute rejectie $(100 \%$ vs. $37.3 \%)$. Daarentegen waren m.b.v. FC $9.8 \%$ van de patiënten antistof-negatief vóór en antistof-positief na transplantatie, $79 \%$ bleef antistof-negatief en $11.2 \%$ van de patiënten was al antistof-positief vóór transplantatie. Patiënten die antistofpositief werden na transplantatie, hadden een lagere transplantaat overleving en vaker rejecties dan beide andere groepen. Door de FC uitslag met post serum te relateren aan dat met current serum, werd de relatieve verandering in fluorescentie ratio (RCFR) bepaald. De $25 \%$ patiënten met de grootste toename in RCFR hadden een vergelijkbaar risico op zowel transplantaat falen als het optreden van acute rejectie als de $9.8 \%$ van de patiënten die antistof-positief waren volgens de standaard definitie. Door antistof-positiviteit te definiëren d.m.v. RCFR kan een grote groep patiënten onderscheiden worden die een verhoogd risico heeft op een ongunstig transplantaat uitkomst. De conclusie van deze studie was dat, ondanks de toegenomen sensitiviteit, de standaard definitie van antistof-positiviteit d.m.v. FC niet resulteert in het onderscheiden van een veel grotere groep patiënten met een verhoogd risico voor rejectie en/of transplantaat verlies dan antistof-positiviteit d.m.v. CDC. Echter d.m.v. onze nieuwe definitie van antistof-positiviteit wordt een veel grotere groep patiënten met verhoogd risico ontdekt. Indien onze bevindingen in een prospectief onderzoek bevestigd worden, kunnen patiënten met een verhoogd risico op transplantaat falen in een vroeg stadium ontdekt en mogelijk behandeld worden door aanpassing van hun therapie.

Sinds de tachtiger jaren is cyclosporine het meest gebruikte immunosuppressivum bijniertransplantaties. De afgelopen jaren zijn nieuwe immunosuppressiva geïntroduceerd, waaronder tacrolimus en mycophenolaat mofetil (MMF). In hoofdstuk 7a wordt een overzicht gegeven van het gebruik van tacrolimus bij niertransplantatie. Behandeling met tacrolimus resulteert, in vergelijking met cyclosporine, in een afname van zowel acute als corticosteroïd-resistente rejecties met $50 \%$. Mogelijk is ook de transplantaat overleving verbeterd. Hoewel beide middelen een vergelijkbaar veiligheidsprofiel hebben, zijn er belangrijke verschillen in bijwerkingen. Overmatige tandvleesgroei en overmatige haargroei worden m.n. gerapporteerd by cyclosporine. Patiënten met tacrolimus hebben een beter cardiovasculair risico profiel (minder vaak een hoge bloeddruk en minder vaak een 
hoog cholesterolgehalte). Een verhoogd bloedsuikergehalte komt echter vaker voor bij patiënten die tacrolimus gebruiken.

Tacrolimus wordt meestal gebruikt in combinatie met steroïden. Met name deze combinatie met MMF is sterk werkzaam. Er bestaan weinig data over de farmacokinetiek van MMF in ontvangers van een getransplanteerd orgaan die tacrolimus gebruiken. In hoofdstuk $\mathbf{7 b}$ hebben wij in een multicenter trial de farmacokinetiek van MMF in combinatie met tacrolimus bestudeerd. Aangetoond werd dat de farmacokinetiek van tacrolimus onveranderd was in aanwezigheid van MMF. De dosering MMF kan echter in loop van de tijd na transplantatie verminderd worden om de blootstelling aan zijn farmacologisch actieve vorm, mycophenolzuur, stabiel te houden.

In hoofdstuk 7c worden richtlijnen voor het doseren en hanteren van tacrolimus in patiënten met een niertransplantatie gegeven. Over het algemeen ontvangen patiënten, met uitzondering van sterk geïmmuniseerden, dezelfde basale immunosuppressie. Gebaseerd op onze ervaringen, kan de immunosuppressie voor iedere patiënt aan zijn/haar specifieke klinische situatie aangepast worden. Een voorstel voor 'maatwerk' immunosuppressie wordt gegeven.

De uitkomst van niertransplantatie wordt bepaald door vele factoren. In hoofdstuk 8 hebben wij onderzocht welke immunologische en niet-immunologische factoren de uitkomst parameters beïnvloeden in een cohort van 346 post-mortale niertransplantaties welke zijn verricht in het academisch ziekenhuis te Maastricht. Alle patiënten gebruikten cyclosporine. D.m.v. multivariaat analyse werden immunologische en niet-immunologische factoren geanalyseerd op hun relatie met klinische uitkomst parameters. Behalve acute rejectie en tranplantaat overleving, zijn de onderzochte parameters: patiënt overleving, de incidentie en duur van het vertraagd opgang komen van de nierfunctie na transplantatie (DGF), opnameduur gedurende het le jaar na transplantatie, en de 1-jaars nierfunctie (kreatinine klaring). De invloed van histocompatibiliteits factoren was matig. Matchen voor sommige HLA loci resulteerde in een betere uitkomst voor de parameters rejectie en 1-jaars kreatinine klaring. Het percentage panel-reactieve antistoffen en het eerder getransplanteerd zijn waren andere immunologische factoren welke DGF en korte termijn patiënt- en transplantaat overleving beïnvloeden. Buiten de histocompatibiliteit waren andere factoren (zowel donor, preservatie, als ontvanger gerelateerd) betrokken bij de korte- en lange termijn uitkomst parameters. Sommigen zouden beïnvloed kunnen worden door een verandering in management van donor en ontvanger. Met name preventie van DGF door de koude ischemie tijd te reduceren en het veranderen van de manier van dialyseren direct vóór transplantatie zou de uitkomst van niertransplantatie kunnen verbeteren. 
In hoofdstuk 9 worden de resultaten van de studie bediscussieerd in relatie tot bestaande immunologische en klinische strategieën in niertransplantatie. Enkele voorstellen voor aanpassingen van deze strategieën worden gemaakt. 


\section{Dankwoord}

Promotieonderzoek is niet alleen het maken van 'het boekje'. Tijdens het verrichten van het onderzoek en het opschrijven hiervan, leer je jezelf beter kennen en leer je nieuwe vaardigheden. Vele mensen staan je hierbij in raad en daad bij, niet alleen als het onderzoek loopt, maar ook tijdens perioden van moedeloosheid of wanhoop.

Allereerst mijn promotor, Hans van Hooff. Beste Hans, naast promotor was je ook mijn opleider en ben je een naaste collega. De bestudeerde onderwerpen liggen je na aan het hart en zijn deels een voortzetting van je eigen onderzoek. Bedankt voor de adviezen en support de afgelopen periode. Je leidraad is: Onderzoek behoort primair ten dienste van patiënten te staan en begint met goede patiëntenzorg. Ik ben er zeker van dat wij nog vele onderzoekingen samen zullen uitvoeren.

Co-promotor betekent letterlijk mede-promotor. Als dit voor iemand opgaat, is het wel Ella van den Berg-Loonen. Beste Ella, naast Hans ben jij de constante factor geweest. Vele onderzoekingen zijn bij jou op het laboratorium opgezet en uitgevoerd. Je wilde er een 'echt' proefschrift van maken. Dit heeft mij de nodige zweetdruppels en (dreigende) wanhoop gekost, en jou vele flessen wijn aan de tafel in je 'oude' keuken, wanneer je weer een onleesbare versie van een artikel probeerde te verhelderen en redigeren. Hartelijk dank voor je steun en toewijding en voor de mogelijkheid als clinicus een kijkje te mogen nemen in het heilige der heiligen, jouw laboratorium. Je hebt gelijk gehad met je opmerking, dat een promovendus alle bepalingen zelf moet (kunnen) uitvoeren en niet alleen een 'opschrijver' is van werk dat door anderen is verricht. Ik hoop nog valak van je kritische blik en correctievermogen gebruik te mogen maken bij gezamenlijke nieuwe projecten.

Fred Nieman wil ik bedanken voor zijn pogingen om wederom een arts de hogere doelen van de statistiek te laten doorgronden. Fred, de basis lijk ik langzaamaan onder de knie te krijgen, maar voor MANOVA's en dat soort analyses, die in iedere nieuwe softwareversie weer anders heten, blijf ik bij je langskomen. Een mens is en blijft stommer dan die 'stomme computer' die weer eens niet doet wat je wilt, ondanks de steeds 'intelligentere' chips.

De dames en enkele heer op het laboratorium voor Weefseltypering bedank ik voor hun hulp bij het aanleren van pipetteren, afdraaien, en opwerken van cellen en sera, en voor het screening en kruizen. Hoe jullie telkens uit hetzelfde cupje ingevroren cellen een groter aantal levende lymfocyten konden overhouden dan ik, zal voor mij altijd wel een raadsel blijven. Zonder de anderen tekort te willen doen, noem ik met name Miriam, Rianne, Marian en Judith. Diana, als secretaresse van Ella ben je altijd een 'buffer' geweest in de goede betekenis van het woord: de stoten heb je opgevangen en verzacht doorgegeven in beide richtingen. Daarnaast ben jij degene geweest die bij de ingezonden manuscripten de 'finishing touch' gaf aan de lay-out. Dit heeft zeker bijgedragen aan de acceptatie van deze manuscripten door de tijdschriften.

De mensen op het laboratorium voor speciêle hematologie bedank ik voor hun introductie in de flow cytometry en voor hun hulp bij het valideren van de FC kruisproeven. 
De vroegere en huidige leden van de werkgroep Nefrologie, Karel, Frank, Jeroen, Jos, Elly, Stijn en Eugenie, bedank ik voor hun plezierige samenwerking, zowel op klinisch gebied als voor hun steun bij mijn onderzoek. Ik hoop dat transplantatie immunologie iets minder onbegrijpelijk is geworden dan dat het was.

Desirée, Luciënne, Anke en Etiênne, als vroegere en huidige secretaresses van de werkgroep Nefrologie bedank ik jullie voor je inzet, maar vooral voor het praatje/luisterend oor. Zonder een goede basis lukt niets.

Ditzelfde geldt voor de verpleging van afdeling D5, zonder twijfel de beste afdeling van het ziekenhuis om (als het toch moet) als patiēnt te liggen. Jullie hebben al die jaren een constante hoge kwaliteit gewaarborgd en een plezierige sfeer gecreëerd voor patiënten en artsen. Dit heeft zeker bijgedragen aan de goede resultaten van de niertransplantatie te Maastricht.

In de eindfase heb ik, zoals zo velen bij de Interne Geneeskunde, mogen meemaken welk een kunde en inzet Tiny Wouters heeft bij de 'afwerking' van het proefschrift. Het uiterlijk is het halve werk en dit is grotendeels te danken aan Tiny. Ik hoop Tiny, dat jij ook volgend millennium je o zo vertrouwde WP5.1 format voor 'Interne proefschriften' kunt blijven gebruiken. Beiden weten wij, dat niets lekkerder typt, dan witte letters op een blauwe achtergrond met sneltoetsen i.p.v. de muis, zeker met drop, koekjes (en voor sommigen een peuk) onder handbereik.

De leden van de beoordelingscommissie. Prof. dr. Kootstra (voorzitter), Prof. dr. Bruggeman, Prof. dr. Claas, Prof. dr. Donckerwolcke en Prof. dr. Opelz, bedank ik voor hun bereidwilligheid om het manuscript te beoordelen en de plezierige samenwerking in de afgelopen jaren, welke ik hoop voort te zetten in de toekomst. Een speciaal woord wil ik richten tot Prof. Kootstra. Gauke, zoals het al snel werd, wat me het meest aan je opviel, is je grenzeloze optimisme. Indien wij in de le week van een nieuw jaar 2 transplantaties hadden verricht, gaf jouw hiërogliefencomputer volgens mij al aan dat wij dat jaar $104(52 \times 2)$ transplantaties zouden halen. Hoewel het aantal niertransplantaties in Maastricht is opgelopen tot \pm 60 per jaar, is er nog veel optimisme en inzet nodig om jouw aantal te halen. Bedankt voor je samenwerking en inzet voor nierpatiènten.

Prof. dr. G Opelz. You are one of the important people of transplantation immunology. Part of the work in this thesis was based on your contributions on the role of transfusion and HLA matching. I'm very honored that you were willing to be one of the reviewer's of my thesis. Thank you.

Mijn ouders bedank ik voor hun warmte en de mogelijkheid en steun om Geneeskunde te studeren. Jullie hebben mij destijds de mogelijkheid gegeven; ik hoop dat dit jullie inspanningen een beetje goedmaakt.

Alle familieleden en vrienden die cen niet-aflatende belangstelling hebben getoond: Het boekje is klaar, nu en niet volgend jaar.

Lest best, Marian en Coen. Marian, zonder een thuis kan je niet leven. Toen wij in 1990 voor een tweetal jaren naar Maastricht vertrokken vonden wij een LAT-relatie niet de 
oplossing. Ruim 9 jaren later denk ik nog steeds dat wij de goede keuze hebben gemaakt. Samen hebben wij in Maastricht een thuis gecreềerd, waarin een warme plaats is voor onze Coen. Bedankt voor je onvoorwaardelijke steun en geloof dat het boekje eens af zou komen. Het naar de studeerkamer sturen om te analyseren/schrijven heeft resultaat gehad. Zonder anderen tekort te doen kan ik dan ook zeggen: Zonder jou was het niet gelukt. Coen, promotie-onderzoek doen is asociaal. Gelukkig was ik een eind op weg toen je kwam. Nu is het tijd voor de schommel in de tuin en andere activiteiten.

Ook deze steen is verlegd in de rivier op aarde, het water zal anders gaan dan voorheen........ 


\title{
Curriculum Vitae
}

\author{
Maarten Christiaans
}

22-7-1960 Born in Utrecht

1972-1978 Gymnasium-B, St. Bonifatius College in Utrecht.

1978-1985 Study Medicine at the Medical Faculty of the University of Utrecht.

1981Bachelor degree cum laude.

1984Doctoral degree cum laude.

1985 Medical degree.

1985 Senior house officer department of Cardiology at the St. Antonius Hospital in Nieuwegein.

1986-1990 Training in Internal Medicine at the St. Antonius Hospital in Nieuwegein (Dr. CEM de Maat, later Dr. OJATh Meuwissen).

1990-1991 Continuation of training in Internal Medicine at the University Hospital Maastricht (Prof.dr. JA Flendrig).

1991 Registration as internist.

1991-1993 Training in Nephrology at the section Nephrology and Transplantation (Prof.dr. JP van Hooff) of the University Hospital Maastricht. 1993Registration as nephrologist.

Since 1991: Staff member of the department of Internal Medicine and 'chef de clinique' of the section Nephrology and Transplantation. University Hospital Maastricht. 


\section{Publicatielijst}

Roodnat JI, Christiaans MHL, Nugteren-Huying WM, van der Schroeff JG, van der Zouwen P, Stricker BHC. Weening JJ, Chang PC. Akute Niereninsuffizienz bei der Behandlung der Psoriasis mit Fumarsaure-Estern. Schweiz Med Wochenschr 1989; 119: 826-30.

Roodnat JI, Christiaans MHL, Nugteren-Huying WM, van der Schroeff JG, Chang PC. Acute nierinsufficientie bij patienten behandeld met fumaarzuuresters wegens psoriasis. Ned Tijdschr Geneeskd 1989; 133: 2623-6.

Christiaans MHL, van den Berg-Loonen PM, Peltenburg HG, Leunissen KML, van Hooff JP. More favorable clinical course in kidney allograft recipients due to HLAB+DR matching. Transplant Proc. 1991; 23: 2674.

Christiaans MHL, Peltenburg HG, Niemann FHM, Kootstra G, Lavrijssen ATJ, van den Berg-Loonen PM, Tiebosch A, Leunissen KML, van Hooff JP. The impact of ischemic lesions in the donor kidney, donor age, recipient age and HLA (A,B,C,DR,DQ) matching on clinical course after kidney transplantation. Transpl Int 1992; 5 (suppl.1): S627-8.

Christiaans MHL, van den Berg-Loonen PM, Nieman FHM, van Hooff JP. Very low incidence of rejection due to $\operatorname{HLA}-(\mathrm{A})+\mathrm{B}+\mathrm{DQ}$ matching. Transplant Proc 1992; 24: 244 I-2.

Christiaans MHL, van den Berg-Loonen PM, Nieman FHM, van Hooff JP. Cadaveric kidney exchange on the basis of HLA-matching is already cost-effective in the first year after grafting. Transplant Proc 1993; 25: 1685-6.

Kraat YJ, Christiaans MHL, Nieman FHM, van den Berg-Loonen PM, van Hooff JP, Bruggeman CA. Increased frequency of CMV infection in HLA-DR7 matched renal allograft recipients (Letter). Lancet 1993; 341 (i): 494-5.

Kraat YJ, Christiaans MHL, Nieman FHM, van den Berg-Loonen PM, van Hooff JP, Bruggeman CA. Risk factors for cytomegalovirus infection and disease in renal transplant recipients: HLA-DR7 and triple therapy. Transpl Int 1994; 7; 362-7.

Peek D, Kootstra G, Christiaans MHL, van Hooff JP. Renal graft thrombosis (Letter). Transplantation 1995; 60: 311 .

Christiaans M, van den Berg-Loonen E, ten Haaft A, Nieman F, van Hooff J. Effect of flow cytometry, complement-dependent cytotoxicity, and auto crossmatch on cadaveric renal transplant outcome. Transplant Proc 1995; 27; 1028-30.

Schleibner S, Krauss M, Wagner K, Erhard J, Christiaans M, van Hooff J, Buist L, Mayer D. FK506 versus Cyclosporin A in the prevention of renal allograft rejection European pilot study: six-week results. Transpl Int 1995; 8; 86-90. 
Spiering W, Christiaans MHL, de Leeuw PW, van Hooff JP. Influence of ACE inhibition on haemoglobin and haematocrit in essential hypertensive patients (Letter). Nephrol Dial Transplant 1995; 10: 1254.

Kraat YJ, Stals FS, Christiaans MHL, Lazorotto T, Landini MP, Bruggeman CA. IgM antibody detection of ppUL80a and ppUL32 by immunoblotting: An early parameter for recurrent cytomegalovirus infection in renal transplant recipients. J Med Virol 1996; 48: 289-94.

Christiaans MHL, Overhof R, ten Haaft A, Nieman F, van Hooff JP, van den BergLoonen PM. No advantage of flow cytometry crossmatch over complement-dependent cytotoxicity in immunologically well-documented renal allograft recipients. Transplantation 1996; 62: 1341-7.

European Tacrolimus Multicenter Renal Study Group (Mayer AD, Dmitrewski J, Squifflet JP, Bessen T, Grabensee B, Klein B, Eigler FW, Heemann U, Pichlmayr R, Behrend M, Vanrenterghem Y, Donck J, van Hooff J, Christiaans M, Morales JM, Andres A, Johnson RWG, Short C, Buchholz B, Rehmert N, Land W, Schleibner S, Forsythe JLR, Talbot E, Neumayer HH, Hauser I. Ericzon BG, Brattstrōm C, Claesson K, Mühlbacher F, Pohanka E). Multicenter randomized trial comparing tacrolimus (FK506) and cyclosporine in the prevention of renal allograft rejection. Transplantation 1997; 64: 436-43.

Blok MJ, Goossens VJ, Vanherle SJV. Top B, Tacken N, Middeldorp JM, Christiaans MHL, van Hooff JP, Bruggeman CA. Diagnostic value of monitoring human cytomegalovirus late pp67 mRNA expression in renal-allograft recipients by nucleic acid sequence-based amplification. J Clin Microbiol 1998; 36: 1341-6

Christiaans MHL, Overhof-de Roos R, Nieman F, van Hooff JP, van den Berg-Loonen PM. Donor-specific antibodies after transplantation by flow cytometry: relative change in fluorescence ratio most sensitive risk factor for renal graft survival. Transplantation 1998; $65: 427-33$.

Undre $\mathrm{N}$ for the European Tacrolimus Multicentre Renal study group (Mayer AD, Dmitrewski J, Squifflet JP, Bessen T, Grabensee B, Klein B, Eigler FW, Heemann U, Pichlmayr R, Behrend M, Vanrenterghem Y, Donck J, van Hooff J, Christiaans M, Morales JM, Andres A, Johnson RWG, Short C, Buchholz B, Rehmert N, Land W, Schleibner S, Forsythe JLR, Talbot E, Neumayer HH, Hauser I, Ericzon BG. Brattström C. Claesson K, Mühlbacher F, Pohanka E). Factors affecting the pharmacokinetics of tacrolimus in the first year after renal transplantation. Transplant Proc 1998; 30: 1261-3.

van Duijnhoven E, Christiaans M, Schàfer A, Undre N, van Hooff J. Tacrolimus dosing requirements in diabetic and nondiabetic patients calculated from pretransplantation data. Transplant Proc 1998; 30: 1266-7.

van Duijnhoven E. Christiaans M, Stevenson P, Undre N, van Hooff J. The effect of breakfast on the oral bioavailability of tacrolimus in diabetic and nondiabetic patients before transplantation. Transplant Proc 1998: 30: 1268-70. 
Christiaans M, van Duijnhoven E, Beysens T, Undre N, van Hooff J. The effect of breakfast on the oral bioavailability of tacrolimus and changes in pharmacokinetics at different times post-transplant in renal transplant recipients. Transplant Proc 1998; 30: 1271-3.

Undre N, van Hooff J, Christiaans M, Vanrenterghem Y, Donck J, Heeman U, Kohnle M, Zanker Z, Land W, Morales JM, Schäfer A. Pharmacokinetics of FK506 and mycophenolic acid after the administration of a FK 506-based regimen in combination with mycophenolate mofetil in kidney transplantation. Transplant Proc 1998; 30: 1299-302.

Richards E, Schleibner S, Talbot D, and the European Tacrolimus Multicentre Renal study group (Mayer AD, Dmitrewski J, Squifflet JP, Bessen T, Grabensee B, Klein B, Eigler FW, Heemann U, Pichlmayr R, Behrend M, Vanrenterghem Y, Donck J, van Hooff J, Christiaans M, Morales JM, Andres A, Johnson RWG, Short C, Buchholz B, Rehmert N, Land W, Schleibner S, Forsythe JLR, Talbot E, Neumayer HH, Hauser I, Ericzon BG, Brattström C, Claesson K, Mühlbacher F, Pohanka E), An exploratory analysis of prognostic factors for patient outcome during the first year following renal transplantation. Transplant Proc 1998: 30: 1386-8.

Blok MJ, Christiaans MHL, Goossens VJ, van Hooff JP, Top B, Middeldorp JM, Bruggeman CA. Evaluation of a new method for early detection of active cytomegalovirus infections. A study in kidney transplant recipients. Transpl Int 1998; 11 (Suppl 1); S107-9.

van Hooff JP, Christiaans M, van Duijnhoven E. Glucose metabolism, insulin and Cpeptide secretion in the first two years after renal transplantation: comparison between tacrolimus and ciclosporin. In: Tacrolimus in organ transplantation: Prevention and Treatment of allograft rejections. Eds: Thiel G, Mosimann F; 1998 Pabst, Lengerich. ISBN: $3-933151-42-2$.

Undre NA, van Hooff J, Christiaans M, Vanrenthergem Y, Donck J, Heeman U, Kohnle M, Zanker B, Land W, Morales JM, Andrés A, Schăfer A, Stevenson P. Low systemic exposure to tacrolimus correlates with acute rejection. Transplant Proc 1999; 31: 296-8.

Blok MJ, Lautenschlager I, Christiaans MHL, van Hooff JP, Goossens VJ, Middeldorp JM, Sillekens P, Ramon A, Hockerstedt K, Bruggeman CA. Nucleid acid sequence-based amplification (NASBA): a new technique for monitoring cytomegalovirus infection in transplant recipients. Transplant Proc 1999; 31: 308-9.

Christiaans MHL, van Hooff JP, Nieman F, van den Berg-Loonen EM. HLA-DR matched transfusions. Development of donor-specific $\mathrm{T}$ - and $\mathrm{B}$-cell antibodies and renal allograft outcome. Transplantation 1999; 67: 1029-35.

Blok MJ, Christiaans MHL, Goossens VJ, van Hooff JP, Middeldorp JM, Bruggeman CA. Early detection of human cytomegalovirus infection after kidney transplantation by nucleic acid sequence-based amplification. Transplantation 1999; 67: 1274-7. 
van den Ham ECH, Kooman JP, Christiaans MHL, Nieman FHM, van Kreel BK, Heidendal GAK, van Hooff JP. Body composition in renal transplant patients: Bioimpedance analysis compared to isotope dilution, dual energy X-ray absorptiometry, and antropometry. J Am Soc Nephrol 1999; 10: 1067-79.

Goossens VJ, Blok MJ, Christiaans MHL, Sillekens P, Middeldorp JM, Bruggeman CA. Early detection of cytomegalovirus in renal transplant recipients: comparison of PCR, NASBA, pp65-antigenemia and viral culture. Transplant Proc (in press).

Christiaans MHL, Nieman F, van Hooff JP, van den Berg-Loonen EM. HLA-class I and II antibodies by ELISA and CDC before and after transplantation. Transplantation (in press).

van den Ham ECH, Kooman JP, Christiaans MHL, van Hooff JP. Relation between steroid dose, body composition and physical activity in renal transplant patients. Transplantation (in press). 



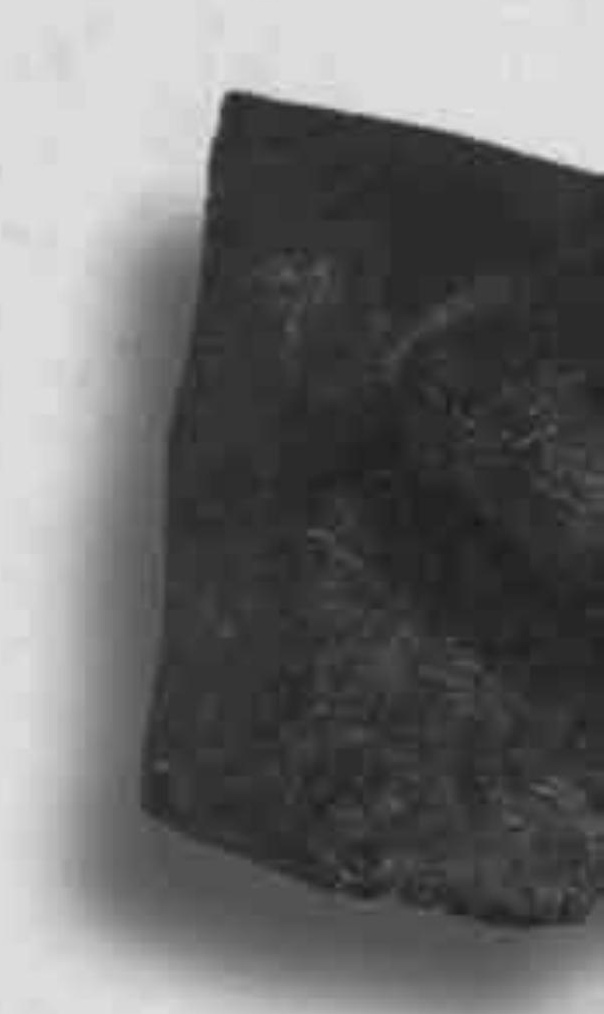

